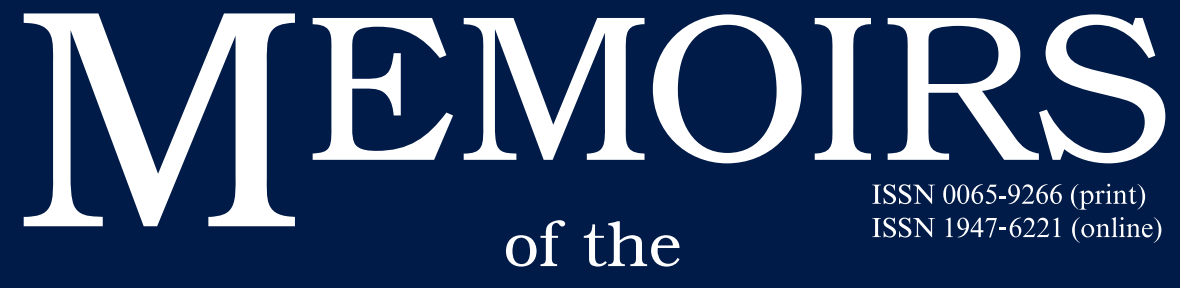

American Mathematical Society

Number 1218

\title{
Perihelia Reduction and Global Kolmogorov Tori in the Planetary Problem
}

Gabriella Pinzari 

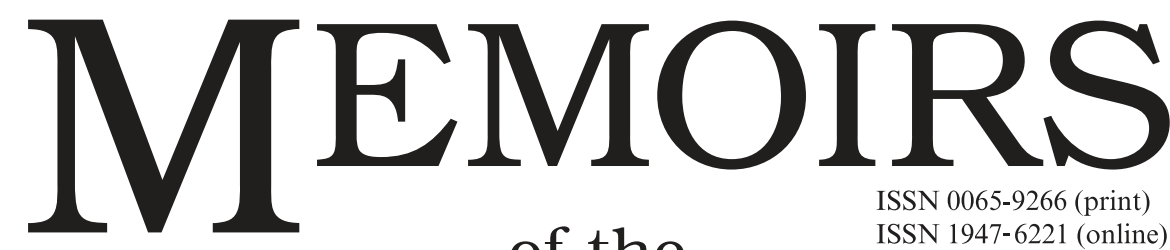

of the

ISSN 0065-9266 (print)

ISSN 1947-6221 (online)

American Mathematical Society

Number 1218

\title{
Perihelia Reduction and Global Kolmogorov Tori in the Planetary Problem
}

\author{
Gabriella Pinzari
}




\section{Library of Congress Cataloging-in-Publication Data}

Names: Pinzari, Gabriella, 1966- author.

Title: Perihelia reduction and global Kolmogorov tori in the planetary problem / Gabriella Pinzari.

Description: Providence, RI : American Mathematical Society, 2018. | Series: Memoirs of the American Mathematical Society, ISSN 0065-9266 ; number 1218 | "September 2018. Volume 255. Number 1218 (first of 7 numbers)." | Includes bibliographical references.

Identifiers: LCCN 2018040533 | ISBN 9781470441029 (alk. paper)

Subjects: LCSH: Celestial mechanics. | Planetary theory. | Differential equations, Partial.

Classification: LCC QB351 .P527 2018 | DDC 521-dc23

LC record available at https://lccn.loc.gov/2018040533

DOI: https://doi.org/10.1090/memo/1218

\section{Memoirs of the American Mathematical Society}

This journal is devoted entirely to research in pure and applied mathematics.

Subscription information. Beginning with the January 2010 issue, Memoirs is accessible from www.ams.org/journals. The 2018 subscription begins with volume 251 and consists of six mailings, each containing one or more numbers. Subscription prices for 2018 are as follows: for paper delivery, US\$999 list, US\$799.20 institutional member; for electronic delivery, US\$879 list, US $\$ 703.20$ institutional member. Upon request, subscribers to paper delivery of this journal are also entitled to receive electronic delivery. If ordering the paper version, add US $\$ 20$ for delivery within the United States; US $\$ 80$ for outside the United States. Subscription renewals are subject to late fees. See www.ams.org/help-faq for more journal subscription information. Each number may be ordered separately; please specify number when ordering an individual number.

Back number information. For back issues see www.ams.org/backvols.

Subscriptions and orders should be addressed to the American Mathematical Society, P. O. Box 845904, Boston, MA 02284-5904 USA. All orders must be accompanied by payment. Other correspondence should be addressed to 201 Charles Street, Providence, RI 02904-2213 USA.

Copying and reprinting. Individual readers of this publication, and nonprofit libraries acting for them, are permitted to make fair use of the material, such as to copy select pages for use in teaching or research. Permission is granted to quote brief passages from this publication in reviews, provided the customary acknowledgment of the source is given.

Republication, systematic copying, or multiple reproduction of any material in this publication is permitted only under license from the American Mathematical Society. Requests for permission to reuse portions of AMS publication content are handled by the Copyright Clearance Center. For more information, please visit www.ams.org/publications/pubpermissions.

Send requests for translation rights and licensed reprints to reprint-permission@ams.org

Excluded from these provisions is material for which the author holds copyright. In such cases, requests for permission to reuse or reprint material should be addressed directly to the author(s). Copyright ownership is indicated on the copyright page, or on the lower right-hand corner of the first page of each article within proceedings volumes.

Memoirs of the American Mathematical Society (ISSN 0065-9266 (print); 1947-6221 (online)) is published bimonthly (each volume consisting usually of more than one number) by the American Mathematical Society at 201 Charles Street, Providence, RI 02904-2213 USA. Periodicals postage paid at Providence, RI. Postmaster: Send address changes to Memoirs, American Mathematical Society, 201 Charles Street, Providence, RI 02904-2213 USA.

(C) 2018 by the American Mathematical Society. All rights reserved.

This publication is indexed in Mathematical Reviews ${ }^{\circledR}$, Zentralblatt MATH, Science Citation

Index ${ }^{\circledR}$, Science Citation Index ${ }^{T M}$-Expanded, ISI Alerting Services ${ }^{S M}$, SciSearch ${ }^{\circledR}$, Research Alert ${ }^{\circledR}$, CompuMath Citation Index ${ }^{\circledR}$, Current Contents ${ }^{\circledR} /$ Physical, Chemical 8 Earth Sciences. This publication is archived in Portico and CLOCKSS.

Printed in the United States of America.

( The paper used in this book is acid-free and falls within the guidelines established to ensure permanence and durability.

Visit the AMS home page at https://www.ams.org/

$10987654321 \quad 232221201918$ 


\section{Contents}

Chapter 1. Background and results Acknowledgments

Chapter 4. Global Kolmogorov tori in the planetary problem 29

4.1. A domain of holomorphy 29

4.2. A normal form for the planetary problem 31

4.3. A "multi-scale" KAM Theorem and proof of Theorem A 33

Chapter 5. Proofs

5.1. Normalization of fast angles

5.2. Secular normalizations 41

Appendix A. Computing the domain of holomorphy 63

A.1. On the analyticity of the solution of Kepler equation

A.2. Proof of Proposition $4.2 \quad 65$

Appendix B. Proof of Lemma 3.2 71

Appendix C. Checking the non-degeneracy condition 75

Appendix D. Some results from perturbation theory 77

D.1. A multi-scale normal form theorem

D.2. A slightly-perturbed integrable system 86

Appendix E. More on the geometrical structure of the $\mathcal{P}$-coordinates, compared to Deprit's coordinates 87

Bibliography 91 
This is a free offprint provided to the author by the publisher. Copyright restrictions may apply. 


\begin{abstract}
We prove the existence of an almost full measure set of $(3 n-2)$-dimensional quasi-periodic motions in the planetary problem with $(1+n)$ masses, with eccentricities arbitrarily close to the Levi-Civita limiting value and relatively high inclinations. This extends previous results, where smallness of eccentricities and inclinations was assumed. The question had been previously considered by V. I. Arnold (1963) in the 60s, for the particular case of the planar three-body problem, where, due to the limited number of degrees of freedom, it was enough to use the invariance of the system by the $\mathrm{SO}(3)$ group.

The proof exploits nice parity properties of a new set of coordinates for the planetary problem, which reduces completely the number of degrees of freedom for the system (in particular, its degeneracy due to rotations) and, moreover, is well fitted to its reflection invariance. It allows the explicit construction of an associated close to be integrable system, replacing Birkhoff normal form, common tool of previous literature.
\end{abstract}

Received by the editor February 21, 2015 and, in revised form, February 9, 2016.

Article electronically published on June 25, 2018.

DOI: https://doi.org/10.1090/memo/1218

2010 Mathematics Subject Classification. Primary 34C20, 70F10, 37J10, 37J15, 37J40; Secondary 34D10, 70F07, 70F15, 37J25, 37J35.

Key words and phrases. Canonical coordinates, Jacobi's reduction, Deprit's reduction, Perihelia reduction, symmetries, quasi-periodic motions, Arnold's theorem on the stability of planetary motions.

This research has been financially supported partly (up to February 28, 2016) by ERC IdeasProject 306414 "Hamiltonian PDEs and small divisor problems: a dynamical systems approach" and partly (since March 1, 2016) by the ERC Project 677793 "Stable and Chaotic Motions in the Planetary Problem."

The author is affiliated with the Dipartimento di Matematica "T. Levi-Civita", via Trieste 63, 35131 Padova, Italy. Email: gabriella.pinzari@math.unipd.it.

(C)2018 American Mathematical Society 
This is a free offprint provided to the author by the publisher. Copyright restrictions may apply. 


\section{CHAPTER 1}

\section{Background and results}

In recent years, substantial progress on a statement by Vladimir Igorevich Arnold concerning the stability of the planetary system has been achieved $[\mathbf{2}, \mathbf{9}, \mathbf{1 4}$, $20,22,23,27,33$.

It sounds as follows.

"For the majority of initial conditions under which the instantaneous orbits of the planets are close to circles lying in a single plane, perturbation of the planets on one another produces, in the course of an infinite interval of time, little change on these orbits provided the masses of the planets are sufficiently small. [...] In particular [...] in the n-body problem there exists a set of initial conditions having a positive Lebesgue measure and such that, if the initial positions and velocities of the bodies belong to this set, the distances of the bodies from each other will remain perpetually bounded." [2, Chapter III, p. 125].

Solving the differential equations of the motions of the planetary problem, i.e., $n$ planets interacting among themselves and with a star via gravity is, for $n \geq 2$, a problem with ancient roots. This story goes back to Sir Isaac Newton - who brilliantly solved the case of two bodies and then, tackling the analogous one for three bodies, soon realized the necessity of turning to a "perturbative" study (except for naming it a "head ache problem") - passed through investigations by eminent mathematicians like Delaunay, Lagrange, the prize publicly announced by King Oscar II of Sweden and Norway and awarded to Henri Poincaré, but its "solution" is nowadays open. Chaotic and stable regions may coexist $[2,11,17$.

The question received a new mathematical description, and a strong modern endorsement, after A. N. Kolmogorov announced, at the International Congress of Mathematicians of 1954 in Amsterdam, what is now almost unanimously considered the most important result of the last century for dynamical systems: the theorem of conservation of the invariant torus. This breakthrough result, next enriched by substantial contributions by J. Moser and V. I. Arnold himself [1, 22, 26, states that for a generic Hamiltonian system close to an integrable one, i.e., a system of the form

$\mathrm{H}(I, \varphi)=\mathrm{h}(I)+\mu f(I, \varphi) \quad(I, \varphi) \in B \times \mathbb{T}^{N} \quad B \subset \mathbb{R}^{N} \quad \mathbb{T}:=\mathbb{R} /(2 \pi \mathbb{Z}) \quad \mu \ll 1$,

the major part of unperturbed motions survives, after a small perturbation is switched on, provided suitable "non-degeneracy" conditions are verified by the "unperturbed part" h. Moreover, the theory provides precise arithmetic ("diophantine") properties to be verified by the "unperturbed frequencies" $\omega_{*}=\partial \mathrm{h}\left(I_{*}\right)$, so that they will be preserved in the full system.

In 1962, V. I. Arnold, extending Kolmogorov's ideas, and looking for an application to the planetary problem, at the International Congress of Mathematicians of 
Stockholm, announced the theorem of stability of planetary motions quoted above. In 1965 Kolmogorov and Arnold were awarded the Lenin Prize for their studies on the stability of the planetary problem - but the story was not finished there.

In order to introduce the results of this paper, we highlight basic facts of this story and its continuation, referring the reader to $[\mathbf{5}, \mathbf{1 0}, \mathbf{1 6}, \mathbf{2 8}, 29$ for more notices.

The planetary problem is close to the integrable problem of $n$ uncoupled two-body problems, where each planet interacts separately with the sun. The mutual interactions among planets are regarded as a perturbing function, the smallness of which is ruled by the planets' masses. However, as a perturbed system, the planetary problem has a limiting degeneracy. Its associated integrable system (the two-body problem) is "super-integrable": it has more integrals than degrees of freedom. At a technical level, the limiting degeneracy is exhibited by the disappearance of degrees of freedom in the unperturbed part. Therefore, continuing the unperturbed motions to a positive measure set of quasi-periodic trajectories might, in general, be not possible, in absence of further informations on the perturbing function.

Arnold found, for the planetary problem, a brilliant solution to the problem of the limiting degeneracy. This led him to add to the assumptions and assertions that are proper of perturbation theories (e.g., "the masses of the planets are sufficiently small", "set of initial conditions having a positive Lebesgue measure", "the distances ... will remain perpetually bounded") a further requirement of smallness of eccentricities and inclinations of the unperturbed Keplerian ellipses ("the instantaneous orbits of the planets are close to circles lying in a single plane"). Let's summarize Arnold's ideas.

Choosing, as Arnold did, Poincaré coordinates [30 (see, also [2 Ch. III, §2], or, e.g., [8, 15]), the system takes the usual close to be integrable form

$$
\mathrm{H}_{\mathcal{P}_{\text {oi }}}=\mathrm{h}_{\mathrm{Kep}}+\mu f_{\mathcal{P}_{\text {oi }}},
$$

where $\mu$ is a small parameter related to the planetary masses, but the unperturbed "Keplerian" part $\mathrm{h}_{\mathrm{Kep}}(\Lambda)$ depends on only $n$ action variables $\Lambda=\left(\Lambda_{1}, \cdots, \Lambda_{n}\right)$ (related to the semi-major axes of the instantaneous Keplerian ellipses), out of an overall of $3 n$ degrees of freedom. The perturbing function, $f_{\mathcal{P} \text { oi }}$, on the other hand, depends on all the coordinates: the actions $\Lambda$, their conjugated angles $\ell=\left(\ell_{1}, \cdots, \ell_{n}\right)$ (proportional to the areas of the elliptic sectors spanned by the planets), and, moreover, on some other coordinates $(\mathrm{p}, \mathrm{q})=\left(\mathrm{p}_{1}, \cdots, \mathrm{p}_{2 n}, \mathrm{q}_{1}, \cdots, \mathrm{q}_{2 n}\right), 4 n$-dimensional, related to those ("secular") quantities (eccentricities, inclinations, nodes and perihelia of the ellipses) that in the unperturbed problem stay fixed, and for this reason do not appear in $h_{\text {Kep }}$.

It is of great help that the averaged perturbing function (with respect to the angles

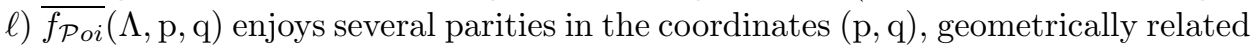
to its invariance by rotations and reflections with respect to the coordinate planes. The "secular origin" $(\mathrm{p}, \mathrm{q})=0$, corresponding to all the planets moving on cocentric circles in the same plane, turns out to be an elliptic equilibrium point for the averaged perturbing function, for any value of $\Lambda$.

Arnold brilliantly argued to exploit this circumstance to his purpose. By Birkhoff theory, one might think to switch to another set of canonical coordinates $(\Lambda, \widetilde{\ell}, \widetilde{p}, \widetilde{q})$, analogous to Poincaré's coordinates, possibly defined only for $(\widetilde{\mathrm{p}}, \widetilde{\mathrm{q}})$ in a small neighborhood of radius $\varepsilon$ around the origin, such that the Hamiltonian of the system, or, 
more precisely, its $\tilde{\ell}$-averaged ("secular") perturbing function $\overline{f_{\mathcal{B} i r}}$, takes a "normalized form" : it is a polynomial, $\overline{f_{\mathcal{B} i r, t r}}$, of some degree greater or equal than two in the combinations ("degenerate actions") $\tau_{i}=\frac{\widetilde{\mathrm{p}}_{i}^{2}+\widetilde{\mathrm{q}}_{i}^{2}}{2}, i=1, \cdots, 2 n$, plus a remainder with a higher order. Roughly, Arnold projected to solve the limiting degeneracy by conjugating the planetary system to a new system, whose unperturbed part was just the truncated, normalized Hamiltonian

$$
\mathrm{h}_{\mathrm{Kep}}+\mu \overline{f_{\mathcal{B} i r, t r}}
$$

so as to recover the standard set up of KAM theory. With these ideas in mind, he proved the following impressive result and next applied it to the planar three-body problem. It states that stable trajectories occupy a positive measure set of the phase space, and are more and more dense closer to the elliptic equilibrium. Hence, the smaller eccentricities and inclinations are, the larger the number of stable motions is.

'The Fundamental Theorem" (V. I. Arnold, 2]) If the Hessian matrix of h and the matrix of the coefficients of the second-order term in $\tau_{i}$ in $\overline{f_{\mathcal{B i r}}}$ ("torsion", or "second-order Birkhoff invariants") do not vanish identically, and if $\mu$ is suitably small with respect to $\varepsilon$, the system affords a positive measure set $\mathcal{K}_{\mu, \varepsilon}$ of quasiperiodic motions in phase space such that its density goes to 1 as $\varepsilon \rightarrow 0$.

Arnold perfectly knew that, in order to apply the Fundamental Theorem to the problem in space, one should previously treat an unpleasant fact: one of the first order Birkhoff invariants vanishes identically. He was aware that the reason for this first-order degeneracy was to be sought into the existence of two non-commuting integrals, the two horizontal components of the total angular momentum of the systems. If, apparently, a vanishing eigenvalue strongly violates the construction of the normalized system (a deeper analysis of the symmetries of the perturbing function $[\mathbf{8}, 25$, however, shows that the identically vanishing eigenvalue is not a real obstruction), a major problem definitely prevents the application of the Fundamental Theorem: an infinite number of coefficients of any order of the (formal) Birkhoff series vanishes identically, among which one entire row and a column in the torsion matrix, which so is identically singular, and the reason is again the invariance by rotations. The proof of this generalized degeneracy is in $\mathbf{8}$. We recall here that even Herman had raised a question about the degeneracy of torsion [20, p. 24].

We do not know weather Arnold was aware of the infinite degeneracy of the normalized system (he did not even mention the vanishing of torsion in his paper). He however suggested two different strategies for the three- and the many-body case, of which he provided very few and somewhat controversial details. As for the three-body problem (his ideas for the many-body case will be recalled a few below), he proposed to reduce the integrals (hence, the number of degrees of freedom) of the system by switching to a system of canonical coordinates going back to the XIX century, worked out by Jacobi and Radau 21,32, which in literature go under the name of Jacobi reduction of the nodes. The idea was later completely developed by P. Robutel [33], who, in a deeply quantitative study, checked the non-degeneracy assumptions required by the Fundamental Theorem.

Finding a system of canonical coordinates that do the job of Jacobi reduction of the nodes when the number of bodies is more than three has been a central difficulty for a long time 2, 25. At this respect, Arnold sadly commented: "In the case of 
more than three bodies there is no such elegant method [as Jacobi reduction of the nodes] of reducing the number of degrees of freedom." [2, Ch. III, §5.5, p. 141].

Exactly twenty years later, F. Boigey and A. Deprit refuted this sentence [3, 12. They indeed were able to extend Jacobi-Radau reduction to the four-body and general problem, respectively. It should be remarked, anyway, that, while the works by Jacobi, Radau and Boigey provide canonical coordinates on suitable submanifolds of the phase space, the one by Deprit is more general and clarifying, since it provides a set of canonical coordinates for the whole phase space and allows us to recover his predecessors by restriction.

The utility of Boigey-Deprit's coordinates was not suddenly clear. Neither Boigey nor Deprit ever provided any motivation of their study, or foresaw applications. The only application that is known to the author up to 2008, concerning indeed Deprit's coordinates, stands in a paper by Ferrer and Osácar, in the 90s, to the three body problem [18. But this case is not really exhaustive, since for three bodies Deprit's and Jacobi-Radau's coordinates coincide. A reason why Boigey-Deprit's coordinates have been forgotten so long might be that, for more than three bodies, they actually have a less natural aspect, compared to the classical case of Jacobi. A sort of "hierarchical" structure in the geometry of Deprit's coordinates discouraged the author himself, who, at the end of his paper, declared: "Whether the new phase variables are practical in the general theory of perturbation is an open question. At least, for planetary theories, the answer is likely to be in the negative. But finding a natural system of coordinates for eliminating the nodes in a planetary cluster was not the intention of this note." [12, p. 194].

In the meantime, in 2004, the first general proof of Arnold's stability statement appeared. It was by Jacques Féjoz, who completed investigations by the late Michael Herman [14 - but the different procedure that Herman had in mind did not rely on the necessity of handling, explicitly, good coordinates. Indeed, Herman conceived a proof based, besides on a "twist-less" KAM theory going back to H. Russmann [34, on indirect arguments of Lagrangian intersections in order to bypass the so-called "secular resonances". See [10] for more details.

In 2008, Boigey-Deprit's coordinates were rediscovered by the author [27, in a slightly different, "planetary" form. The rediscovery was motivated by the purpose of realizing Arnold's program (i.e., applying the Fundamental Theorem quoted above directly to the planetary Hamiltonian) in the general case, so as to obtain a detailed information about the tori frequencies, the measure of the invariant set and the symplectic structure of the phase space. The utility of Boigey-Deprit's coordinates became suddenly clear: switching (in order to overcome certain singularities of the chart) to a regularized version, called "RPS" coordinates, (acronym standing for "Regular, Planetary and Symplectic"), allowed them to derive the Birkhoff normal form of the planetary problem, to prove its non-degeneracy, and hence to complete the application of the Fundamental Theorem to the general problem. These results have been published in $6, \mathbf{6}, \mathbf{9}$.

Qualitatively, RPS coordinates are very different from JRBD (Jacobi-Radau-BoigeyDeprit); rather, they are more similar to Poincaré coordinates. The mentioned parities and the elliptic equilibrium of the averaged system are still present in the RPS-averaged system. But, as an advantage with respect to Poincaré coordinates, 
the RPS perform 11 a "partial reduction" of the rotation symmetry - in contrast with JRBD coordinates, which reduce "fully". This way, all the degeneracies of the Birkhoff series mentioned above are removed at once, and the non-degeneracy assumptions of the Fundamental Theorem may be checked.

We like to recall now Arnold's strategy for the many-body case: more than forty years earlier, he foresaw to construct a system of coordinates analogous to RPS, via a Taylor series in Poincaré coordinates [2, Ch III, §5, n. 5, p. 141].

Indeed, both the reduction of the nodes and this latter reduction are available whatever the number of bodies is.

The possibility of switching from Delaunay-Poincaré to the more fruitful JRBD, or even RPS coordinates, is an effect of the limiting degeneracy. This gives in fact the opportunity of remixing coordinates related to secular quantities, and, simultaneously, keeping the Keplerian term $\mathrm{h}_{\text {Kep }}$ unvaried.

Following this idea, in this paper, we show that other systems of coordinates may be determined for the planetary problem which, as well as JRBD and RPS coordinates, are well adapted to overcome the degeneracy due to rotations, and, moreover, enjoy some different properties.

We present a full reduction, which we call $\mathcal{P}$-map, or perihelia reduction. It refines JRBD coordinates in two respects.

Firstly, the $\mathcal{P}$-map is well defined in the case of the planar problem, while JRBD coordinates are not. Everyone knows, in fact, that the starting point for the RadauJacobi reduction is the so-called "line of the nodes", the straight line determined by the intersection between the planes of the two orbits. When the orbits of the two planets belong to the same plane, this is not defined. A similar circumstance arises for Boigey-Deprit's coordinates, since their construction relies on certain straight lines in the space, which again lose their meaning in case of co-planarity.

The proof of Arnold's theorem given in [9, 27] is not affected by such singularity, since, as said, it relies on RPS coordinates, which, at the expense of one more degree of freedom, are well defined for co-planar motions - in that case they reduce to the classical Poincaré coordinates.

It has its consequences when one wants to compare results for the fully reduced systems, in space or in the plane. The singularity of the chart does not allow one to state that motions in the spatial problem with minimum number of independent frequencies starting with very small inclinations stay close to the corresponding planar motions. Notwithstanding further studies appearing in [28], where this problem is partially overcome (via the construction of regular coordinates for coplanar motions defined locally), it would be nice, in principle, to handle a global system of action-angle coordinates which completely reduces rotations and is shared simultaneously by the planar and the spatial problem.

Secondly, the $\mathcal{P}$-map is well adapted to reflection symmetries of the problem, while JRBD coordinates are not, as discussed in [25, 29.

\footnotetext{
${ }^{1}$ In the framework of the study of canonical coordinates for the planetary system, by "partial reduction", we mean a system of canonical coordinates where a couple of conjugated coordinates consists of integrals (e.g., functions of the three components of the total angular momentum). By "full reduction", we mean a partial reduction where also another integral appears among the coordinates. The terms "partial reduction", "full reduction" have been coined in [25.
} 
Reflection symmetries are parities of the Hamiltonian expressed in Cartesian coordinates. As known, this does not change under arbitrary changes of the signs of positions or momenta coordinates. They are not related to integrals. Therefore, it might be a nice fact, and in general useful for applications, to have a system of coordinates that, after integrals are reduced, parities associated to reflections are maintained. Quite often parities are associated to equilibria, and equilibria to stable motions; an example is provided a few lines below.

We shall apply the $\mathcal{P}$-map by proving a variant of Arnold's stability theorem. We shall face up to a question raised again by Arnold in his fantastic paper on the possibility of removing the constraint on eccentricities and inclinations. He indeed proved that, at least for the planar three-body problem, there is no need to assume their smallness. Rather, it is sufficient that the trajectories of the planets are away enough so as to avoid collisions. He obtained this stronger result by exploiting the convergence of the Birkhoff series associated to the averaged perturbation, a very particular and happy circumstance, due to the few degrees of freedom of the problem.

From the mathematical point of view, the question is whetherstrategies exist for finding stable motions other than the one of exploring the neighborhood of the elliptic equilibrium.

Concerning instead the physical relevance, asteroids or some trans-Neptunian objects have motions with relatively large eccentricities and inclinations and an almost continuous spectrum of frequencies.

Besides the mentioned stronger result by Arnold, some other statements in the same direction have been obtained for the case of the spatial three-body problem and the planar problem with any number of bodies $\mathbf{2 8}$. Here, the measure of the invariant set has been estimated to be larger and larger as the planetary masses and the semi-axes ratios are small, no matter the smallness of the eccentricities and inclinations - the proof relies on an argument of convergence of a significant approximation of the Birkhoff series. Other results in this direction have been announced by J. Féjoz, since late 2013 [13.

Even though the arguments of $[\mathbf{2}, \mathbf{2 8}$ do not apply to the general spatial problem, since no significant approximation of the Birkhoff series associated to the averaged perturbation is integrable, using the $\mathcal{P}$-map, we shall prove the following.

Theorem A. Fix numbers $0<\underline{e}_{i}<\bar{e}_{i}<0.6627 \ldots, i=1, \cdots, n$. There exists a number $\mathrm{N}$ depending only on $n$ and a number $\alpha_{0}$ depending on $\underline{e}_{i}, \bar{e}_{i}$, and $n$ such that, if $\alpha<\alpha_{0}, \mu \leq \alpha^{\mathrm{N}}$, in a domain of planetary motions where the semi-major axes $a_{1}<a_{2}<\cdots<a_{n}$ are spaced as follows

$$
a_{i}^{-} \leq a_{i} \leq a_{i}^{+} \quad \text { with } \quad a_{i}^{ \pm}:=\frac{a_{1}^{ \pm}}{\alpha^{\frac{1}{3}\left(2^{n+1}-2^{n-i+2}+1-i\right)}}
$$

there exists a positive measure set $\mathcal{K}_{\mu, \alpha}$, the density of which in phase space can be bounded below as

$$
\operatorname{dens}\left(\mathcal{K}_{\mu, \alpha}\right) \geq 1-\left(\log \alpha^{-1}\right)^{\mathrm{p}} \sqrt{\alpha}
$$

consisting of quasi-periodic motions with $3 n-2$ frequencies where the planets' eccentricities $e_{i}$ verify

$$
\underline{e}_{i} \leq e_{i} \leq \bar{e}_{i}
$$


Before we switch to details, a few remarks.

Firstly, the claimed upper bound $0.6627 \ldots$ is classical. It is related to the fact that, as well as in $2, \mathbf{2 8}$, the proof uses the machinery of real-analytic functions. We refer the reader to [24,35] and references therein for general notices. A treatment of the argument, as needed in the present paper, is provided in Chapter A.1.

Secondly, as it may be seen to the choice of $a_{j}^{ \pm}$, the distances among the planets' semi-axes are not of the same order but grow super-exponentially going towards the sun. This resembles a sort of belt arrangement, observed in nature for asteroids. It is possible to prove an analogous result, with increasing distances in the opposite direction.

Thirdly, the result in Theorem A (especially, the claimed growth of $a_{i}^{ \pm}$) may be regarded as an alternative way of solving the problem of the limiting degeneracy without Birkhoff normal form.

\section{Acknowledgments}

The author is indebted to Jacques Féjoz, who let the author know the work by Harrington [19, without which I would never have thought of this application of the $\mathcal{P}$-coordinates. Also, I am deeply grateful to A. Celletti and R. de la Llave, for their interest and for encouraging me with precious advices. Thanks finally to L. Biasco for his interest. 
This is a free offprint provided to the author by the publisher. Copyright restrictions may apply. 


\section{CHAPTER 2}

\section{Kepler maps and the Perihelia reduction}

We introduce the Perihelia reduction, or $\mathcal{P}$-map, in the slightly general context of Kepler maps.

Fix a reference frame $\mathrm{G}_{0}=\left(k^{(1)}, k^{(2)}, k^{(3)}\right)$ in the Euclidean space $E^{3}$. We identify the three chosen directions $k^{(1)}, k^{(2)}, k^{(3)}$ with the triples of coordinates with respect of the system of coordinates established by themselves:

$$
k^{(1)}=\left(\begin{array}{l}
1 \\
0 \\
0
\end{array}\right) \quad k^{(2)}=\left(\begin{array}{l}
0 \\
1 \\
0
\end{array}\right) \quad k^{(3)}=\left(\begin{array}{l}
0 \\
0 \\
1
\end{array}\right) .
$$

Definition 2.1. An ellipse (with a focus in the origin and non-vanishing eccentricity) is a quadruplet $\mathfrak{E}=(a, e, N, P)$, where $a \in \mathbb{R}_{+}$is the semi-major axis, $e \in(0,1)$ is the eccentricity, $N \in \mathbb{R}^{3} \cap S^{2}$ is the normal direction and $P \in N^{\perp} \cap S^{2}$ is the perihelion direction.

Definition 2.2 (Kepler maps). Given $2 n$ positive "mass parameters" $\mathfrak{m}_{1}, \cdots$, $\mathfrak{m}_{n}, \mathfrak{M}_{1}, \cdots, \mathfrak{M}_{n}$, a set $\mathfrak{X} \subset \mathbb{R}^{5 n}$, we say that a map

$$
\mathcal{K}: \quad \mathrm{K}=\left(\mathrm{X}_{\mathcal{K}}, \ell\right) \in \mathcal{D}:=\mathfrak{X} \times \mathbb{T}^{n} \rightarrow\left(y_{\mathcal{K}}, x_{\mathcal{K}}\right) \in \mathcal{C}:=\mathcal{K}(\mathcal{D}) \subset\left(\mathbb{R}^{3}\right)^{n} \times\left(\mathbb{R}^{3}\right)^{n}
$$

where

$$
\begin{array}{ll}
\ell=\left(\ell_{1}, \cdots, \ell_{n}\right), & \left(y_{\mathcal{K}}, x_{\mathcal{K}}\right)=\left(y_{\mathcal{K}}^{(1)}, \cdots, y_{\mathcal{K}}^{(n)}, x_{\mathcal{K}}^{(1)}, \cdots, x_{\mathcal{K}}^{(n)}\right) \\
y_{\mathcal{K}}^{(j)}=y_{\mathcal{K}}^{(j)}\left(\mathrm{X}_{\mathcal{K}}, \ell_{j}\right) & x_{\mathcal{K}}^{(j)}=x_{\mathcal{K}}^{(j)}\left(\mathrm{X}_{\mathcal{K}}, \ell_{j}\right) \quad j=1, \cdots n,
\end{array}
$$

is a Kepler map if there exists an injection

$$
\tau_{\mathcal{K}}: \quad \mathrm{X}_{\mathcal{K}} \in \mathfrak{X} \rightarrow \mathfrak{E}_{\mathcal{K}}=\left(\mathfrak{E}_{1, \mathcal{K}}, \cdots, \mathfrak{E}_{n, \mathcal{K}}\right)
$$

which assigns to any $\mathrm{X}_{\mathcal{K}} \in \mathfrak{X}$ an n-plet $\left(\mathfrak{E}_{1, \mathcal{K}}, \cdots, \mathfrak{E}_{n, \mathcal{K}}\right)$ of (co-focal) ellipses

$$
\mathfrak{E}_{j, \mathcal{K}}=\left(a_{j, \mathcal{K}}, e_{j, \mathcal{K}}, N_{\mathcal{K}}^{(j)}, P_{\mathcal{K}}^{(j)}\right), \quad j=1, \cdots, n
$$

and $\mathcal{K}$ acts in the following way. Letting $Q_{\mathcal{K}}^{(j)}:=N_{\mathcal{K}}^{(j)} \times P_{\mathcal{K}}^{(j)}$, then

$$
x_{\mathcal{K}}^{(j)}=\mathrm{a}_{j, \mathcal{K}} P_{\mathcal{K}}^{(j)}+\mathrm{b}_{j, \mathcal{K}} Q_{\mathcal{K}}^{(j)} \quad y_{\mathcal{K}}^{(j)}=\mathrm{a}_{j, \mathcal{K}}^{\circ} P_{\mathcal{K}}^{(j)}+\mathrm{b}_{j, \mathcal{K}}^{\circ} Q_{\mathcal{K}}^{(j)}
$$

where, if $\zeta_{j, \mathcal{K}}$, the eccentric anomaly, is the solution of Kepler's Equation

$$
\zeta_{j, \mathcal{K}}-e_{j, \mathcal{K}} \sin \zeta_{j, \mathcal{K}}=\ell_{j}
$$


then

$$
\begin{array}{ll}
\mathrm{a}_{j, \mathcal{K}}:=a_{j, \mathcal{K}}\left(\cos \zeta_{j, \mathcal{K}}-e_{j, \mathcal{K}}\right) & \mathrm{b}_{j, \mathcal{K}}:=a_{j, \mathcal{K}} \sqrt{1-e_{j, \mathcal{K}}^{2}} \sin \zeta_{j, \mathcal{K}} \\
\mathrm{a}_{j, \mathcal{K}}^{\circ}:=-\mathfrak{m}_{j} \sqrt{\frac{\mathfrak{M}_{j}}{a_{j, \mathcal{K}}}} \frac{\sin \zeta_{j, \mathcal{K}}}{1-e_{j, \mathcal{K}} \cos \zeta_{j, \mathcal{K}}} & \mathrm{b}_{j, \mathcal{K}}^{\circ}:=\mathfrak{m}_{j} \sqrt{\frac{\mathfrak{M}_{j}\left(1-e_{j, \mathcal{K}}^{2}\right)}{a_{j, \mathcal{K}}}} \frac{\cos \zeta_{j, \mathcal{K}}}{1-e_{j, \mathcal{K}} \cos \zeta_{j, \mathcal{K}}} .
\end{array}
$$

REMARK 2.1. The definition implies that

(i) $\mathcal{K}$ is a bijection of the sets $\mathcal{D}$ and $\mathcal{C}$;

(ii) the angular momenta and the energies 1

$$
\mathrm{C}_{\mathcal{K}}^{(j)}:=x_{\mathcal{K}}^{(j)} \times y_{\mathcal{K}}^{(j)}, \quad \mathrm{H}_{\mathcal{K}}^{(j)}:=\frac{\left\|y_{\mathcal{K}}^{(j)}\right\|^{2}}{2 \mathfrak{m}_{j}}-\frac{\mathfrak{m}_{j} \mathfrak{M}_{j}}{\left\|x_{\mathcal{K}}^{(j)}\right\|}
$$

do not depend on $\ell_{j}$ and are given by

$$
\mathrm{C}_{\mathcal{K}}^{(j)}=\mathfrak{m}_{j} \sqrt{\mathfrak{M}_{j} a_{j, \mathcal{K}}\left(1-e_{j, \mathcal{K}}^{2}\right)} N_{\mathcal{K}}^{(j)}, \quad \mathrm{H}_{\mathcal{K}}^{(j)}=-\frac{\mathfrak{m}_{j} \mathfrak{M}_{j}}{2 a_{j, \mathcal{K}}}
$$

(iii) the couples $\left(y_{\mathcal{K}}^{(j)}, x_{\mathcal{K}}^{(j)}\right)$ verify the system of ODEs

$$
\left\{\begin{array}{l}
\mathfrak{m}_{j} \sqrt{\frac{\mathfrak{M}_{j}}{a_{j, \mathcal{K}}^{3}}} \partial_{\ell_{j}} x_{\mathcal{K}}^{(j)}=y_{\mathcal{K}}^{(j)} \\
\sqrt{\frac{\mathfrak{M}_{j}}{a_{j, \mathcal{K}}^{3}}} \partial_{\ell_{j}} y_{\mathcal{K}}^{(j)}=-\mathfrak{m}_{j} \mathfrak{M}_{j} \frac{x_{\mathcal{K}}^{(j)}}{\left\|x_{\mathcal{K}}^{(j)}\right\|^{3}} .
\end{array}\right.
$$

(iv) Even though canonical maps (with respect to the standard two-form) have a pre-eminent role in Hamiltonian Mechanics, Kepler maps are used also in different contexts in Astronomy, where being canonical is not required. For example, one can consider the Kepler map associated to the "elliptic elements" injection

$$
\tau_{\mathcal{E} e \ell \ell}: \quad(a, e, P, i, \Omega) \rightarrow \mathfrak{E}_{\mathcal{E} e l \ell}
$$

where $a=\left(a_{1}, \cdots, a_{n}\right)$ are the semi-major axes, $e=\left(e_{1}, \cdots, e_{n}\right)$ are the eccentricities, $P=\left(P^{(1)}, \cdots, P^{(n)}\right)$ are the perihelia, $i=\left(i_{1}, \cdots, i_{n}\right)$ are the inclinations, $\Omega=\left(\Omega_{1}, \cdots, \Omega_{n}\right)$ are the nodes' longitudes.

The only known examples up to now of canonical Kepler maps are the classical Delaunay map Del (its definition is recalled in the next Definition 2.5) and the map Dep [7, 27. related to Deprit's coordinates [12, which is recalled in Appendix E. Below, we introduce a new canonical Kepler map.

Definition 2.3 (perihelia reduction, or $\mathcal{P}$-map). We denote as $\mathcal{P}$, and call perihelia reduction, or $\mathcal{P}$-map, the Kepler map

$$
\mathcal{P}: \quad \mathrm{P}=\left(\mathrm{X}_{\mathcal{P}}, \ell\right) \in \mathcal{D}_{\mathcal{P}}=\mathfrak{X}_{\mathcal{P}} \times \mathbb{T}^{n} \rightarrow(y, x) \in \mathbb{R}^{3 n} \times \mathbb{R}^{3 n}
$$

associated to the bijection

$$
\tau_{\mathcal{P}}: \quad \mathrm{X}_{\mathcal{P}}=(\Theta, \chi, \Lambda, \vartheta, \kappa) \in \mathfrak{X}_{\mathcal{P}} \rightarrow\left(\mathfrak{E}_{1}, \cdots, \mathfrak{E}_{n}\right) \ln \mathrm{E}_{\mathcal{P}}=\tau_{\mathcal{P}}\left(\mathfrak{X}_{\mathcal{P}}\right) \subset E^{3 n}
$$

defined by means of Definition 2.4 and Proposition 2.1 below.

\footnotetext{
${ }^{1}$ Here, $\|v\|:=\sqrt{v_{1}^{2}+v_{2}^{2}+v_{3}^{2}}$ denotes the usual Euclidean norm of $v=\left(v_{1}, v_{2}, v_{3}\right) \in \mathbb{R}^{3}$.
} 
Definition 2.4. For a given $\left(\mathfrak{E}_{1}, \cdots, \mathfrak{E}_{n}\right) \subset E^{3} \times \cdots \times E^{3}$, with

$$
\mathfrak{E}_{j}=\left(a_{j}, e_{j}, N^{(j)}, P^{(j)}\right),
$$

and masses $\mathfrak{m}_{1}, \cdots, \mathfrak{m}_{n}, \mathfrak{M}_{1}, \cdots, \mathfrak{M}_{n}$, define

$$
\mathrm{C}_{\mathcal{E}}^{(j)}:=\mathfrak{m}_{j} \sqrt{\mathfrak{M}_{j} a_{j}\left(1-e_{j}^{2}\right)} N^{(j)} \quad \mathrm{S}_{\mathcal{E}}^{(j)}:=\sum_{i=j}^{n} \mathrm{C}_{\mathcal{E}}^{(i)} \quad 1 \leq j \leq n
$$

be the angular momenta associated to $\mathfrak{E}_{j}$ and the $j^{\text {th }}$ partial angular momenta, so that

$$
\mathrm{S}_{\mathcal{E}}^{(1)}=\sum_{i=1}^{n} \mathrm{C}_{\mathcal{E}}^{(i)} \quad \mathrm{S}_{\mathcal{E}}^{(n)}=\mathrm{C}_{\mathcal{E}}^{(n)}
$$

are the total angular momentum and the angular momentum of the last ellipse, respectively. Define the $\mathcal{P}$-nodes

$$
\nu_{j}:=\left\{\begin{array}{ll}
k^{(3)} \times \mathrm{S}_{\mathcal{E}}^{(1)} & j=1 \\
P^{(j-1)} \times \mathrm{S}_{\mathcal{E}}^{(j)} & j=2, \cdots, n
\end{array} \quad \mathrm{n}_{j}:=\mathrm{S}_{\mathcal{E}}^{(j)} \times P^{(j)} \quad j=1, \cdots, n .\right.
$$

Finally, define

$\mathcal{E}_{\mathcal{P}}:=\left\{\left(\left(\mathfrak{E}_{1}, \cdots, \mathfrak{E}_{n}\right) \subset E^{3} \times \cdots \times E^{3}\right): 0<e_{j}<1, \nu_{j} \neq 0 \mathrm{n}_{j} \neq 0 \forall j=1, \cdots, n\right\}$, and, on this set, the map

$$
\tau_{\mathcal{P}}^{-1}: \quad\left(\mathfrak{E}_{1}, \cdots, \mathfrak{E}_{n}\right) \in \mathcal{E}_{\mathcal{P}} \rightarrow \mathrm{X}_{\mathcal{P}} \in \mathfrak{X}_{\mathcal{P}}=\tau_{\mathcal{P}}^{-1}\left(\mathcal{E}_{\mathcal{P}}\right)
$$

where

$$
\mathrm{X}_{\mathcal{P}}=(\Theta, \chi, \Lambda, \vartheta, \kappa) \in \mathbb{R}^{n} \times \mathbb{R}_{+}^{n} \times \mathbb{R}_{+}^{n} \times \mathbb{T}^{n} \times \mathbb{T}^{n}
$$

with

$$
\begin{aligned}
& \Theta=\left(\Theta_{0}, \cdots, \Theta_{n-1}\right), \quad \vartheta=\left(\vartheta_{0}, \cdots, \vartheta_{n-1}\right) \\
& \chi=\left(\chi_{0}, \cdots, \chi_{n-1}\right), \quad \kappa=\left(\kappa_{0}, \cdots, \kappa_{n-1}\right) \\
& \Lambda=\left(\Lambda_{1}, \cdots, \Lambda_{n}\right)
\end{aligned}
$$

defined via the following formulae:

$$
\begin{aligned}
& \Theta_{j-1}:=\left\{\begin{array}{lr}
Z:=\mathrm{S}_{\mathcal{E}}^{(1)} \cdot k^{(3)} \\
\mathrm{S}_{\mathcal{E}}^{(j)} \cdot P^{(j-1)}
\end{array} \quad \vartheta_{j-1}:=\left\{\begin{array}{lr}
\zeta:=\alpha_{k^{(3)}}\left(k^{(1)}, \nu_{1}\right) & j=1 \\
\alpha_{P^{(j-1)}}\left(\mathrm{n}_{j-1}, \nu_{j}\right) & 2 \leq j \leq n
\end{array}\right.\right. \\
& \chi_{j-1}:=\left\{\begin{array}{lr}
\mathrm{G}:=\left\|\mathrm{S}_{\mathcal{E}}^{(1)}\right\| \\
\left\|\mathrm{S}_{\mathcal{E}}^{(j)}\right\|
\end{array} \quad \kappa_{j-1}:=\left\{\begin{array}{lr}
\mathfrak{g}:=\alpha_{\mathrm{S}_{\mathcal{E}}^{(1)}}\left(\nu_{1}, \mathrm{n}_{1}\right) & j=1 \\
\alpha_{\mathrm{S}_{\mathcal{E}}^{(j)}}\left(\nu_{j}, \mathrm{n}_{j}\right) & 2 \leq j \leq n
\end{array}\right.\right.
\end{aligned}
$$$$
\Lambda_{j}:=\mathfrak{M}_{j} \sqrt{\mathfrak{m}_{j} a_{j}} .
$$ 
Proposition 2.1. Let $\mathfrak{X}_{\mathcal{P}}$ be the subset of $\mathbb{R}^{n} \times \mathbb{R}_{+}^{n} \times \mathbb{R}_{+}^{n} \times \mathbb{T}^{n} \times \mathbb{T}^{n}$ defined by the following inequalities

$$
\begin{aligned}
& \sqrt{\chi_{i-1}^{2}+\chi_{i}^{2}-2 \Theta_{i}^{2}+2 \sqrt{\left(\chi_{i}^{2}-\Theta_{i}^{2}\right)\left(\chi_{i-1}^{2}-\Theta_{i}^{2}\right)} \cos \vartheta_{i}}<\Lambda_{i} \\
& \left(\chi_{i-1}-\chi_{i}, \vartheta_{i}\right) \neq(0, \pi) \quad 0<\chi_{n-1}<\Lambda_{n} \quad i=1, \cdots, n-1
\end{aligned}
$$

and

$$
\left|\Theta_{0}\right|<\chi_{0} \quad\left|\Theta_{i}\right|<\min \left(\chi_{i-1}, \chi_{i}\right) \quad i=1, \cdots, n-1 .
$$

The map $\tau_{\mathcal{P}}^{-1}$ is a bijection of $\mathcal{E}_{\mathcal{P}}$ onto $\mathfrak{X}_{\mathcal{P}}$. The formulae of the inverse map

$$
\begin{gathered}
\tau_{\mathcal{P}}: \quad \mathrm{X}_{\mathcal{P}}=(\Theta, \chi, \Lambda, \vartheta, \kappa) \in \mathcal{D}_{\mathcal{P}} \rightarrow \mathfrak{E}_{\mathcal{P}}=\left(\mathfrak{E}_{1, \mathcal{P}}, \cdots, \mathfrak{E}_{n, \mathcal{P}}\right) \in \mathcal{E}_{\mathcal{P}} \\
\mathfrak{E}_{j, \mathcal{P}}=\left(a_{j, \mathcal{P}}, e_{j, \mathcal{P}}, N_{\mathcal{P}}^{(j)}, P_{\mathcal{P}}^{(j)}\right)
\end{gathered}
$$

are as follows. Let $\iota_{1}, \cdots, \iota_{n}, \mathrm{i}_{1}, \cdots, \mathrm{i}_{n} \in(0, \pi)$ be defined via

$$
\cos \iota_{j}=\frac{\Theta_{j-1}}{\chi_{j-1}}, \quad \cos i_{j}:=\frac{\Theta_{j}}{\chi_{j-1}}, \quad 1 \leq j \leq n
$$

(with $\Theta_{n}:=0$, so that $\mathrm{i}_{n}=\frac{\pi}{2}$ ) and $\mathcal{T}_{1}, \cdots, \mathcal{T}_{n}, \mathcal{S}_{1}, \cdots, \mathcal{S}_{n} \in \mathrm{SO}(3)$ via

$$
\mathrm{T}_{j}:=\mathcal{R}_{3}\left(\vartheta_{j}\right) \mathcal{R}_{1}\left(\iota_{j}\right) \quad \mathrm{S}_{j}:=\mathcal{R}_{3}\left(\kappa_{j}\right) \mathcal{R}_{1}\left(\mathrm{i}_{j}\right), \quad 1 \leq j \leq n
$$

and let

$$
\mathrm{C}_{\mathcal{P}}^{(j)}:=\mathrm{T}_{1} \mathrm{~S}_{1} \cdots \mathrm{T}_{j-1} \mathrm{~S}_{j-1} \mathrm{~T}_{j}\left(\chi_{j-1} k^{(3)}-\chi_{j} \mathrm{~S}_{j} \mathrm{~T}_{j+1} k^{(3)}\right)
$$

with $\chi_{n}:=0$, so that

$$
\left\|\mathrm{C}_{\mathcal{P}}^{(j)}\right\|= \begin{cases}\sqrt{\chi_{j-1}^{2}+\chi_{j}^{2}-2 \Theta_{j}^{2}+2 \sqrt{\left(\chi_{j}^{2}-\Theta_{j}^{2}\right)\left(\chi_{j-1}^{2}-\Theta_{j}^{2}\right)} \cos \vartheta_{j}} & j=1, \cdots, n-1 \\ \chi_{n-1} & j=n .\end{cases}
$$

Then $\mathrm{C}_{\mathcal{P}}^{(j)}=\mathrm{C}_{\mathcal{E}}^{(j)} \circ \tau_{\mathcal{P}}$ and

$$
a_{j, \mathcal{P}}=\frac{1}{\mathfrak{M}_{j}}\left(\frac{\Lambda_{j}}{\mathfrak{m}_{j}}\right)^{2} \quad e_{j, \mathcal{P}}=\sqrt{1-\frac{\left\|\mathrm{C}_{\mathcal{P}}^{(j)}\right\|^{2}}{\Lambda_{j}^{2}}}
$$

$$
N_{\mathcal{P}}^{(j)}=\frac{\mathrm{C}_{\mathcal{P}}^{(j)}}{\left\|\mathrm{C}_{\mathcal{P}}^{(j)}\right\|} \quad P_{\mathcal{P}}^{(j)}=\mathrm{T}_{1} \mathrm{~S}_{1} \cdots \mathrm{T}_{j} \mathrm{~S}_{j} k^{(3)} .
$$

REMARK 2.2.

(i) From $\mathrm{C}_{\mathcal{P}}^{(j)}=\mathrm{C}_{\mathcal{E}}^{(j)} \circ \tau_{\mathcal{P}}$, (2.4), (2.5) and (2.25), there follows that $\mathrm{C}_{\mathcal{P}}^{(j)}=$ $x_{\mathcal{P}}^{(j)} \times y_{\mathcal{P}}^{(j)}$.

(ii) $P_{\mathcal{P}}^{(j)} \perp N_{\mathcal{P}}^{(j)}$. Indeed, using the definitions,

$$
\begin{aligned}
\mathrm{C}_{\mathcal{P}}^{(j)} \cdot P_{\mathcal{P}}^{(j)} & =\chi_{j-1} k^{(3)} \cdot\left(\mathrm{S}_{j} k^{(3)}\right)-\mathcal{T}_{j+1} \chi_{j} k^{(3)} \cdot\left(k^{(3)}\right) \\
& =\chi_{j-1} \cos \iota_{j}-\chi_{j} \cos i_{j+1} \\
& =0 .
\end{aligned}
$$

(iii) $\mathrm{S}_{\mathcal{P}}^{(j)}:=\mathrm{S}_{\mathcal{E}}^{(j)} \circ \tau_{\mathcal{P}}=\sum_{i=j}^{n} \mathrm{C}_{\mathcal{P}}^{(i)}=\chi_{j-1} \mathrm{~T}_{1} \mathrm{~S}_{1} \cdots \mathrm{T}_{j-1} \mathrm{~S}_{j-1} \mathrm{~T}_{j} k^{(3)}$.

We shall prove that 
Theorem 2.1. The $\mathcal{P}$-map preserves the standard 2-form

$$
\sum_{j=1}^{n} d y_{\mathcal{P}}^{(j)} \wedge d x_{\mathcal{P}}^{(j)}=\sum_{i=1}^{n}\left(d \Theta_{i-1} \wedge d \vartheta_{i-1}+d \chi_{i-1} \wedge d \kappa_{i-1}+d \Lambda_{i} \wedge d \ell_{i}\right) .
$$

REMARK 2.3. Actually, we shall prove a finer result: the change $\phi_{\mathcal{D} e \ell}^{\mathcal{P}}:=$ $\mathcal{D} e \ell^{-1} \circ \mathcal{P}$ which relates the $\mathcal{P}$-coordinates to the classical Delaunay coordinates (see the Definition 2.5) is homogeneous-canonical (compare Lemma 2.6).

Proof of Proposition 2.1. The formula for $a_{j, \mathcal{P}}$ in (2.16) is immediate from the definition of $\Lambda_{j}$. Postponing to below that $\mathrm{C}_{\mathcal{P}}^{(j)}:=\mathrm{C}_{\mathcal{E}}^{(j)} \circ \tau_{\mathcal{P}}$ has the expression in (2.16) (in turn this implies (2.17), the formula for $N^{(j)}$ and the one for $e_{j, \mathcal{P}}$ in $(2.18)$ ), we check that the image set $\tau_{\mathcal{P}}^{-1}\left(\mathcal{E}_{\mathcal{P}}\right)$ is included in the domain $\mathfrak{X}_{\mathcal{P}}$ defined by inequalities (2.12), (2.13). From the formula for $e_{j, \mathcal{P}}$ in (2.18), we have that conditions $0<e_{j, \mathcal{P}}<1$ for all $j=1, \cdots, n$ correspond to relations in (2.12). Note that the first condition in the second line of (2.12) is equivalent to $e_{j, \mathcal{P}} \neq 1$, as one sees rewriting

$$
\left\|\mathbf{C}_{\mathcal{P}}^{(j)}\right\|^{2}=\left(\sqrt{\chi_{j-1}^{2}-\Theta_{j}^{2}}-\sqrt{\chi_{j}^{2}-\Theta_{j}^{2}}\right)^{2}+2 \sqrt{\left(\chi_{j}^{2}-\Theta_{j}^{2}\right)\left(\chi_{j-1}^{2}-\Theta_{j}^{2}\right)}\left(1+\cos \vartheta_{j}\right) .
$$

Next, recalling the definitions of $\Theta_{0}, \chi_{0}$ in (2.11), and noticing the relations

$$
\Theta_{j}=\mathrm{S}_{\mathcal{E}}^{(j+1)} \cdot P^{(j)}=\left(\mathrm{S}_{\mathcal{E}}^{(j)}-\mathrm{C}_{\mathcal{E}}^{(j)}\right) \cdot P^{(j)}=\mathrm{S}_{\mathcal{E}}^{(j)} \cdot P^{(j)} \quad j=1, \cdots, n-1,
$$

we immediately see that conditions $\nu_{i} \neq 0 \neq \mathrm{n}_{i}$ imply (2.13). We have so checked what we wanted.

Now it remains to check the formula for $\mathrm{C}_{\mathcal{P}}^{(j)}$ in (2.16) and the one for $P_{\mathcal{P}}^{(j)}$ in (2.18), for any $\mathrm{X}_{\mathcal{P}} \in \mathfrak{X}_{\mathcal{P}}$. To this end, we consider the following chain of vectors

$$
\begin{aligned}
& k^{(3)} \rightarrow \mathrm{S}_{\mathcal{E}}^{(1)} \rightarrow P^{(1)} \rightarrow \cdots \rightarrow \mathrm{S}_{\mathcal{E}}^{(j)} \rightarrow P^{(j)} \rightarrow \cdots \rightarrow P^{(n)} \\
& \Downarrow \quad \Downarrow \quad \begin{array}{llllll} 
& \Downarrow & \Downarrow & \Downarrow & \vdots & \Downarrow
\end{array} \\
& \begin{array}{lllllll}
\nu_{1} & \mathrm{n}_{1} & \vdots & \nu_{j} & \mathrm{n}_{j} & \vdots & \mathrm{n}_{n}
\end{array}
\end{aligned}
$$

where $\nu_{1}, \mathrm{n}_{1}, \cdots, \nu_{n}, \mathrm{n}_{n}$ are the $\mathcal{P}$-nodes in (2.10), given by the skew-product of the two consecutive vectors in the chain.

We associate to this chain of vectors the following chain of frames

$$
\mathrm{G}_{0} \rightarrow \mathrm{F}_{1} \rightarrow \mathrm{G}_{1} \rightarrow \cdots \rightarrow \mathrm{F}_{j} \rightarrow \mathrm{G}_{j} \rightarrow \mathrm{F}_{j+1} \rightarrow \cdots \rightarrow \mathrm{G}_{n}
$$

where $\mathrm{G}_{0}=\left(k^{(1)}, k^{(2)}, k^{(3)}\right)$ is the initial prefixed frame and the frames, while $\mathrm{F}_{i}$, $\mathrm{G}_{i}$ are frames defined via

$$
\mathrm{F}_{j}=\left(\nu_{j}, \cdot, \mathrm{S}^{(j)}\right) \quad \mathrm{G}_{j}=\left(\mathrm{n}_{j}, \cdot, P^{(j)}\right) \quad j=1, \cdots, n .
$$

By construction, each frame in the chain has its first axis coinciding with the intersection of horizontal plane with the horizontal plane of the previous frame (hence, in particular, $\nu_{j} \perp \mathrm{S}^{(j)}$ and $\left.\mathrm{n}_{j} \perp P^{(j)}\right)$. Denote as $\mathrm{T}_{j}$ the rotation matrix which describes the change of coordinates from $\mathrm{G}_{j-1}$ to $\mathrm{F}_{j}$ and as $\mathrm{S}_{j}$ the one from $\mathrm{F}_{j}$ to $\mathrm{G}_{j}$. The matrices $\mathcal{T}_{j}, \mathcal{S}_{j}$ have just the expressions claimed in (2.14), (2.15). 
This follows from the definitions of $(\Theta, \chi, \vartheta, \kappa)$ in (2.11). Then we have the following sequence of transformations

$$
\begin{aligned}
& \begin{array}{llllll}
\mathrm{T}_{1} & \mathrm{~S}_{1} & \ldots & \mathrm{S}_{j} & \ldots & \mathrm{S}_{n}
\end{array} \\
& \mathrm{G}_{0} \rightarrow \mathrm{F}_{1} \rightarrow \mathrm{G}_{1} \rightarrow \cdots \rightarrow \mathrm{F}_{j} \rightarrow \mathrm{G}_{j} \rightarrow \cdots \rightarrow \mathrm{G}_{n}
\end{aligned}
$$

connecting $\mathrm{G}_{0}$ to any other frame in the chain. From this, and the definitions of the frames (2.22), the formulae for $P_{\mathcal{P}}^{(j)}$ in (2.18) and

$$
\mathrm{S}_{\mathcal{P}}^{(j)}=\chi_{j-1} \mathrm{~T}_{1} \mathrm{~S}_{1} \cdots \mathrm{T}_{j-1} \mathrm{~S}_{j-1} \mathrm{~T}_{j} k^{(3)}
$$

follow at once. Hence, also the formulae for $\mathrm{C}_{\mathcal{P}}^{(j)}$, which is given by $\mathrm{C}_{\mathcal{P}}^{(j)}=\mathrm{S}_{\mathcal{P}}^{(j)}-$ $\mathrm{S}_{\mathcal{P}}^{(j+1)}$, with $\mathrm{S}_{\mathcal{P}}^{(n+1)} \equiv 0$.

For the proof of Theorem 2.1. we shall use three auxiliary maps, that we shall denote as $\widetilde{\mathcal{P}}, \widetilde{\mathcal{D} e l}$ and $\mathcal{D} e l$. The map $\widetilde{\mathcal{P}}$ is very closely related to $\mathcal{P} ; \widetilde{\mathcal{D} e l}$ and $\mathcal{D} e l$ are well known: in the literature they are often referred to as (two variants of) Delaunay maps.

The map $\widetilde{\mathcal{P}}$. Define the set

$$
\mathcal{C}_{\widetilde{\mathcal{P}}}:=\left\{(y, x) \in \mathbb{R}^{3 n} \times \mathbb{R}^{3 n}: \quad x^{(j)} \neq 0, \quad \widetilde{\mathrm{n}}_{j}: \neq 0, \quad \widetilde{\nu}_{j} \neq 0 \quad \forall j=1, \cdots, n\right\},
$$

where, for $(y, x) \in \mathbb{R}^{3 n} \times \mathbb{R}^{3 n}$, with $y=\left(y^{(1)}, \cdots, y^{(n)}\right), x=\left(x^{(1)}, \cdots, x^{(n)}\right)$, $x^{(j)} \neq 0$, we let

$$
\widetilde{\nu}_{j}:=\left\{\begin{array}{ll}
k^{(3)} \times \mathrm{S}_{\mathcal{C}}^{(1)} & j=1 \\
\frac{x^{(j-1)}}{\left\|x^{(j-1)}\right\|} \times \mathrm{S}_{\mathcal{C}}^{(j)} & j=2, \cdots, n
\end{array} \quad \widetilde{\mathrm{n}}_{j}:=\mathrm{S}_{\mathcal{C}}^{(j)} \times \frac{x^{(j)}}{\left\|x^{(j)}\right\|}\right.
$$

with $j=1, \cdots, n$ and

$$
\mathrm{C}_{\mathcal{C}}^{(j)}:=x^{(j)} \times y^{(j)}, \quad \mathrm{S}_{\mathcal{C}}^{(j)}:=\sum_{i=j}^{n} \mathrm{C}^{(i)}
$$

Define a map

$$
\widetilde{\mathcal{P}}^{-1}: \quad(y, x) \in \mathcal{C}_{\widetilde{\mathcal{P}}} \rightarrow(\widetilde{\Theta}, \widetilde{\chi}, \widetilde{\mathrm{R}}, \widetilde{\vartheta}, \widetilde{\kappa}, \widetilde{\mathrm{r}}) \in \mathbb{R}^{n} \times \mathbb{R}_{+}^{n} \times \mathbb{R}^{n} \times \mathbb{T}^{n} \times \mathbb{T}^{n} \times \mathbb{R}_{+}^{n}
$$

with

$$
\begin{array}{ll}
\widetilde{\Theta}=\left(\widetilde{\Theta}_{0}, \cdots, \widetilde{\Theta}_{n-1}\right) & \widetilde{\vartheta}=\left(\widetilde{\vartheta}_{0}, \cdots, \widetilde{\vartheta}_{n-1}\right) \\
\widetilde{\chi}=\left(\widetilde{\chi}_{0}, \cdots, \widetilde{\chi}_{n-1}\right) & \widetilde{\kappa}=\left(\widetilde{\kappa}_{0}, \cdots, \widetilde{\kappa}_{n-1}\right) \\
\widetilde{\mathrm{R}}=\left(\widetilde{\mathrm{R}}_{1}, \cdots, \widetilde{\mathrm{R}}_{n}\right) & \widetilde{\mathrm{r}}=\left(\widetilde{\mathrm{r}}_{1}, \cdots, \widetilde{\mathrm{r}}_{n}\right)
\end{array}
$$


via the following formulae:

$$
\begin{aligned}
& \widetilde{\mathrm{R}}_{j}=\frac{y^{(j)} \cdot x^{(j)}}{\left\|x^{(j)}\right\|} \\
& \widetilde{\mathrm{r}}_{j}=\left\|x^{(j)}\right\| \\
& j=1, \cdots, n \\
& \tilde{\chi}_{j-1}=\left\|S_{\mathcal{C}}^{(j)}\right\| \\
& \widetilde{\kappa}_{j-1}=\alpha_{\mathrm{S}_{\mathcal{C}}^{(j)}}\left(\widetilde{\nu}_{j}, \widetilde{\mathrm{n}}_{j}\right) \\
& j=1, \cdots, n \\
& \widetilde{\Theta}_{j-1}=\left\{\begin{array}{l}
\mathrm{S}_{\mathcal{C}}^{(1)} \cdot k^{(3)} \\
\mathrm{S}_{\mathcal{C}}^{(j)} \cdot \frac{x^{(j-1)}}{\left\|x^{(j-1)}\right\|}
\end{array}\right. \\
& \widetilde{\vartheta}_{j-1}= \begin{cases}\alpha_{k^{(3)}}\left(k^{(1)}, \widetilde{\nu}_{1}\right) & j=1 \\
\alpha_{\frac{x^{(j-1)}}{\left\|x^{(j-1)}\right\|}\left(\widetilde{\mathrm{n}}_{j-1}, \widetilde{\nu}_{j}\right)} j=2, \cdots, n .\end{cases}
\end{aligned}
$$

LEMma 2.1. Let $\mathcal{D}_{\widetilde{\mathcal{P}}}$ be the set of $(\widetilde{\Theta}, \widetilde{\chi}, \widetilde{\mathrm{R}}, \widetilde{\vartheta}, \widetilde{\kappa}, \widetilde{\mathrm{r}}) \in \mathbb{R}^{n} \times \mathbb{R}_{+}^{n} \times \mathbb{R}^{n} \times \mathbb{T}^{n} \times \mathbb{T}^{n} \times \mathbb{R}_{+}^{n}$ such that $(\widetilde{\Theta}, \widetilde{\chi}, \widetilde{\vartheta}, \widetilde{\kappa})$ satisfies (2.13), and let $\widetilde{\mathrm{T}}_{j}, \widetilde{\mathrm{S}}_{j}$ and $\mathrm{C}_{\widetilde{\mathcal{P}}}^{(j)}$ be the functions of $(\widetilde{\Theta}, \widetilde{\chi}, \widetilde{\vartheta}, \widetilde{\kappa})$ defined in (2.14) $-(2.16)$, with $(\widetilde{\Theta}, \widetilde{\chi}, \widetilde{\vartheta}, \widetilde{\kappa})$ replacing $(\Theta, \chi, \vartheta, \kappa)$.

The map $\widetilde{\mathcal{P}}^{-1}$ is a bijection from $\mathcal{C}_{\widetilde{\mathcal{P}}}$ onto the set $\mathcal{D}_{\widetilde{\mathcal{P}}}$. Its inverse map

$$
\widetilde{\mathcal{P}}: \quad(\widetilde{\Theta}, \widetilde{\chi}, \widetilde{\mathrm{R}}, \widetilde{\vartheta}, \widetilde{\kappa}, \widetilde{\mathrm{r}}) \in \mathcal{D}_{\widetilde{\mathcal{P}}} \rightarrow\left(y_{\widetilde{\mathcal{P}}}, x_{\widetilde{\mathcal{P}}}\right) \in \mathbb{R}^{n} \times \mathbb{R}^{n}
$$

has the following analytical expression:

$$
\left\{\begin{array}{l}
x_{\widetilde{\mathcal{P}}}^{(j)}:=\widetilde{\mathrm{r}}_{j} \widetilde{\mathrm{T}}_{1} \widetilde{\mathrm{S}}_{1} \cdots \widetilde{\mathrm{T}}_{j} \widetilde{\mathrm{S}}_{j} k^{(3)} \\
y_{\widetilde{\mathcal{P}}}^{(j)}:=\frac{\widetilde{\mathrm{R}}_{j}}{\widetilde{\mathrm{r}}_{j}} x_{\widetilde{\mathcal{P}}}^{(j)}+\frac{1}{\widetilde{\mathrm{r}}_{j}^{2}} \mathrm{C}_{\widetilde{\mathcal{P}}}^{(j)} \times x_{\widetilde{\mathcal{P}}}^{(j)} \quad 1 \leq j \leq n
\end{array}\right.
$$

Moreover, the following relation holds:

$$
\mathrm{C}_{\widetilde{\mathcal{P}}}^{(j)}=\mathrm{C}_{\mathcal{C}}^{(j)} \circ \widetilde{\mathcal{P}}=x_{\mathcal{P}}^{(j)} \times y_{\mathcal{P}}^{(j)} .
$$

Proof. With similar arguments as the ones of the proof of Proposition 2.1] but replacing, in the diagram (2.20), $\mathrm{S}_{\mathcal{E}}^{(j)}$ with $\mathrm{S}_{\mathcal{C}}^{(j)}, P_{\mathcal{P}}^{(j)}$ with $\frac{x^{(j)}}{\left\|x^{(j)}\right\|}$ and the nodes $\nu_{k}, \mathrm{n}_{k}$ with $\widetilde{\nu}_{k}, \widetilde{\mathrm{n}}_{k}$, one finds the formula for $x_{\widetilde{\mathcal{P}}}^{(j)}$ in (2.24), the formula for

$$
\mathrm{S}_{\widetilde{\mathcal{P}}}^{(j)}:=\mathrm{S}_{\mathcal{C}}^{(j)} \circ \widetilde{\mathcal{P}}=\widetilde{\chi}_{j-1} \widetilde{\mathrm{T}}_{1} \widetilde{\mathrm{S}}_{1} \cdots \widetilde{\mathrm{T}}_{j-1} \widetilde{\mathrm{S}}_{j-1} \widetilde{\mathrm{T}}_{j} k^{(3)}
$$

and hence the formula for

$$
\mathrm{C}_{\mathcal{C}}^{(j)} \circ \widetilde{\mathcal{P}}=\mathrm{S}_{\widetilde{\mathcal{P}}}^{(j)}-\mathrm{S}_{\widetilde{\mathcal{P}}}^{(j+1)}=\mathrm{C}_{\widetilde{\mathcal{P}}}^{(j)}
$$

being just the formula for $\mathrm{C}_{\mathcal{P}}^{(j)}$ in $(2.16)$, with $(\Theta, \chi, \vartheta, \kappa)$ replaced by $(\widetilde{\Theta}, \widetilde{\chi}, \widetilde{\vartheta}, \widetilde{\kappa})$. With the same argument as in Remark 2.2 (ii), we see that $x_{\widetilde{\mathcal{P}}}^{(j)} \perp \mathrm{C}_{\tilde{\mathcal{P}}}^{(j)}$. Finally, the formula for $y_{\widetilde{\mathcal{P}}}^{(j)}$ is found taking for $y_{\widetilde{\mathcal{P}}}^{(j)}$ the unique vector verifying

$$
y_{\mathcal{P}}^{(j)} \cdot \frac{x_{\mathcal{P}}^{(j)}}{\left\|x_{\mathcal{P}}^{(j)}\right\|}=\mathrm{R}_{j} \quad x_{\mathcal{P}}^{(j)} \times y_{\mathcal{P}}^{(j)}=\mathrm{C}_{\mathcal{P}}^{(j)} .
$$

LEMMA 2.2. $\widetilde{\mathcal{P}}$ preserves the standard Liouville 1-form:

$$
\sum_{j=1}^{n} y_{\widetilde{\mathcal{P}}}^{(j)} \cdot d x_{\widetilde{\mathcal{P}}}^{(j)}=\sum_{j=1}^{n}\left(\widetilde{\Theta}_{j-1} d \widetilde{\vartheta}_{j-1}+\widetilde{\chi}_{j-1} d \widetilde{\kappa}_{j-1}+\widetilde{\mathrm{R}}_{j} d \widetilde{\mathrm{r}}_{j}\right)
$$


The proof of Lemma 2.2 uses the following easy lemma:

Lemma $2.3(\mathbf{7})$. Let

$$
x=\mathcal{R}_{3}(\theta) \mathcal{R}_{1}(i) \bar{x}, \quad y=\mathcal{R}_{3}(\theta) \mathcal{R}_{1}(i) \bar{y}, \quad \mathrm{C}:=x \times y, \quad \overline{\mathrm{C}}:=\bar{x} \times \bar{y},
$$

with $x, \bar{x}, y, \bar{y} \in \mathbb{R}^{3}$. Then,

$$
y \cdot d x=\mathrm{C} \cdot k^{(3)} d \theta+\overline{\mathrm{C}} \cdot k^{(1)} d i+\bar{y} \cdot d \bar{x} .
$$

Proof of Lemma 2.2. We may write

$$
x_{\widetilde{\mathcal{P}}}^{(j)}=\widetilde{\mathrm{T}}_{1} \widetilde{\mathrm{S}}_{1} \cdots \widetilde{\mathrm{T}}_{j} \widetilde{\mathrm{S}}_{j} \tilde{x}^{(j)}, \quad y_{\widetilde{\mathcal{P}}}^{(j)}=\widetilde{\mathrm{T}}_{1} \widetilde{\mathrm{S}}_{1} \cdots \widetilde{\mathrm{T}}_{j} \widetilde{\mathrm{S}}_{j} \tilde{y}^{(j)}, \quad \mathrm{C}_{\widetilde{\mathcal{P}}}^{(j)}=\widetilde{\mathrm{T}}_{1} \widetilde{\mathrm{S}}_{1} \cdots \widetilde{\mathrm{T}}_{j} \widetilde{\mathrm{S}}_{j} \tilde{\mathrm{C}}^{(j)}
$$

where

$$
\begin{aligned}
\tilde{x}^{(j)} & :=\widetilde{\mathrm{r}}_{j} k^{(3)} \quad j=1, \cdots, n-1 \\
\tilde{y}^{(j)} & :=\widetilde{\mathrm{R}}_{j} k^{(3)}+\frac{1}{\widetilde{\mathrm{r}}_{j}} \tilde{\mathrm{C}}^{(j)} \times k^{(3)} \\
\tilde{\mathrm{C}}^{(j)} & :=\widetilde{\chi}_{j-1} \widetilde{\mathcal{S}}_{j}^{-1} k^{(3)}-\widetilde{\chi}_{j} \widetilde{\mathcal{T}}_{j+1} k^{(3)}=\tilde{x}^{(j)} \times \tilde{y}^{(j)}
\end{aligned}
$$

with $\widetilde{\chi}_{n}:=0, \widetilde{\mathcal{S}}_{n}:=$ id. We also let, for $1 \leq k \leq j \leq n$ and $1 \leq i \leq n-1$,

$$
\begin{aligned}
& \hat{\mathrm{C}}_{k}^{(j)}=\widetilde{\mathcal{S}}_{k}\left(\widetilde{\mathcal{T}}_{k+1} \widetilde{\mathcal{S}}_{k+1} \cdots \widetilde{\mathcal{T}}_{j} \widetilde{\mathcal{S}}_{j}\right) \tilde{\mathrm{C}}^{(j)}, \quad \check{\mathrm{C}}_{k}^{(j)}=\widetilde{\mathcal{T}}_{k} \widetilde{\mathcal{S}}_{k} \ldots \widetilde{\mathcal{T}}_{j} \widetilde{\mathcal{S}}_{j} \tilde{\mathrm{C}}^{(j)}, \quad \check{\mathrm{C}}_{j+1}^{(j)}:=\tilde{\mathrm{C}}^{(j)} \\
& \hat{\mathrm{S}}_{k}^{(j)}:=\sum_{m=j}^{n} \hat{\mathrm{C}}_{k}^{(m)}, \quad \check{\mathrm{S}}_{k}^{(j)}:=\sum_{m=j}^{n} \check{\mathrm{C}}_{k}^{(m)}, \quad \check{\mathrm{S}}_{i+1}^{(i)}:=\tilde{\mathrm{C}}^{(i)}+\check{\mathrm{S}}_{i+1}^{(i+1)}
\end{aligned}
$$

where the product $\widetilde{\mathcal{T}}_{k+1} \widetilde{\mathcal{S}}_{k+1} \ldots \widetilde{\mathcal{T}}_{j} \widetilde{\mathcal{S}}_{j}$ is to be replaced with the identity when $k=j$. We have the following identities (implied by $\mathrm{S}^{(j)}=\sum_{k=j}^{n} \mathrm{C}^{(k)}$ ):

$$
\check{\mathrm{S}}_{j}^{(j)}=\sum_{k=j}^{n} \check{\mathrm{C}}_{j}^{(k)}=\widetilde{\chi}_{j-1} \widetilde{\mathcal{T}}_{j} k^{(3)}, \quad \hat{\mathrm{S}}_{j}^{(j)}=\sum_{k=j}^{n} \hat{\mathrm{C}}_{j}^{(k)}=\widetilde{\chi}_{j-1} k^{(3)}, \quad \check{\mathrm{S}}_{i+1}^{(i)}=\widetilde{\chi}_{j-1} \widetilde{\mathcal{S}}_{i}^{-1} k^{(3)} .
$$

Applying Lemma 2.3 repeatedly and using (as it follows from (2.27)

$$
\tilde{y}^{(j)} \cdot d \tilde{x}^{(j)}=\widetilde{\mathrm{R}}_{j} d \widetilde{\mathrm{r}}_{j}
$$

we have, for $1 \leq j \leq n$,

$$
\begin{aligned}
y_{\widetilde{\mathcal{P}}}^{(j)} \cdot x_{\widetilde{\mathcal{P}}}^{(j)}=\sum_{k=1}^{j} & \left(\check{\mathrm{C}}_{k}^{(j)} \cdot k^{(3)} d \widetilde{\vartheta}_{k-1}\right. \\
& \left.\quad+\hat{\mathrm{C}}_{k}^{(j)} \cdot k^{(1)} d \widetilde{\iota}_{k}+\hat{\mathrm{C}}_{k}^{(j)} \cdot k^{(3)} d \widetilde{\kappa}_{k-1}+\check{\mathrm{C}}_{k+1}^{(j)} \cdot k^{(1)} \widetilde{\mathrm{i}_{k}}\right)+\widetilde{\mathrm{R}}_{j} d \widetilde{\mathrm{r}}_{j}
\end{aligned}
$$

where, as in the proof of Lemma 2.1, $\widetilde{\iota}_{j}, \widetilde{\mathrm{i}}_{j}$ denote the functions $\iota_{j}, \mathrm{i}_{j}$ in (2.14), with $\Theta_{i}, \chi_{i}$ replaced by $\widetilde{\Theta}_{i}, \widetilde{\chi}_{i}$. Note that we have used $d \widetilde{\mathrm{i}}_{n} \equiv 0$, since, by definition, $\widetilde{\mathrm{i}}_{n}=\frac{\pi}{2}$. Taking the sum over $j=1, \cdots, n$,

$$
\begin{gathered}
\sum_{j=1}^{n} y_{\widetilde{\mathcal{P}}}^{(j)} \cdot d x_{\widetilde{\mathcal{P}}}^{(j)}=\sum_{j=1}^{n} \check{\mathrm{S}}_{j}^{(j)} \cdot k^{(3)} d \widetilde{\vartheta}_{j-1}+\hat{\mathrm{S}}_{j}^{(j)} \cdot k^{(1)} d \widetilde{\iota}_{j}+ \\
\hat{\mathrm{S}}_{j}^{(j)} \cdot k^{(3)} d \widetilde{\kappa}_{j-1}+\check{\mathrm{S}}_{j+1}^{(j)} \cdot k^{(1)} \widetilde{\mathrm{d}}_{j}+\sum_{j=1}^{n} \widetilde{\mathrm{R}}_{j} d \widetilde{\mathrm{r}}_{j} .
\end{gathered}
$$

In view of (2.28) and of the definitions in (2.14)-(2.15), we then find (2.26). 
The map $\widetilde{\mathcal{D e l}}$. The map

$\widetilde{\mathcal{D} e \ell}: \quad(\widetilde{\mathrm{H}}, \widetilde{\Gamma}, \widetilde{\mathrm{R}}, \widetilde{\mathrm{h}}, \widetilde{\mathrm{g}}, \widetilde{\mathrm{r}}) \in \mathcal{D}_{\widetilde{\mathcal{D} e \ell}} \rightarrow\left(y_{\widetilde{\mathcal{D} e \ell}}, x_{\widetilde{\mathcal{D} e \ell}}\right) \in \mathbb{R}^{3 n} \times \mathbb{R}^{3 n}$

is defined on the set

$$
\begin{aligned}
& \mathcal{D}_{\widetilde{\mathcal{D e l}}}:=\left\{(\widetilde{\mathrm{H}}, \widetilde{\Gamma}, \widetilde{\mathrm{R}}, \widetilde{\mathrm{h}}, \widetilde{\mathrm{g}}, \widetilde{\mathrm{r}})=\left(\widetilde{\mathrm{H}}_{1}, \cdots, \widetilde{\mathrm{H}}_{n}, \widetilde{\Gamma}_{1}, \cdots, \widetilde{\Gamma}_{n}, \widetilde{\mathrm{R}}_{1}, \cdots, \widetilde{\mathrm{R}}_{n}, \widetilde{\mathrm{h}}_{1}, \cdots, \widetilde{\mathrm{h}}_{n}\right.\right. \\
&\left.\quad \widetilde{\mathrm{g}}_{1}, \cdots, \widetilde{\mathrm{g}}_{n}, \widetilde{\mathrm{r}}_{1}, \cdots, \widetilde{\mathrm{r}}_{n}\right) \in \mathbb{R}^{3 n} \times \mathbb{T}^{2 n} \times \mathbb{R}_{+}^{n}: \quad \widetilde{\mathrm{r}}_{j}>0, \quad \widetilde{\Gamma}_{j}>0, \quad \frac{\left|\widetilde{\mathrm{H}}_{j}\right|}{\widetilde{\Gamma}_{j}}<1 \\
&\quad \forall j=1, \cdots, n\}
\end{aligned}
$$

via the following formulae:

$$
x_{\widetilde{\mathcal{D} e \ell}}^{(j)}:=\mathrm{R}_{3}\left(\widetilde{\mathrm{h}}_{\mathrm{j}}\right) \mathcal{R}_{1}\left(\widetilde{i}_{j}\right) \bar{x}_{\widetilde{\mathcal{D} e \ell}}^{(j)}, \quad y_{\overline{\mathcal{D} e \ell}}^{(j)}:=\mathrm{R}_{3}\left(\widetilde{\mathrm{h}}_{\mathrm{j}}\right) \mathcal{R}_{1}\left(\widetilde{i}_{j}\right) \bar{y}_{\overline{\mathcal{D} e \ell}}^{(j)}
$$

where

$$
\begin{aligned}
\widetilde{i}_{j} & :=\cos ^{-1} \frac{\widetilde{\mathrm{H}}_{j}}{\widetilde{\Gamma}_{j}} \in(0, \pi) \\
\bar{x}_{\widetilde{\mathcal{D} e \ell}}^{(j)} & :=\widetilde{\mathrm{r}}_{j} \cos \widetilde{\mathrm{g}}_{j} k^{(1)}+\widetilde{\mathrm{r}}_{j} \sin \widetilde{\mathrm{g}}_{j} k^{(2)} \\
\bar{y}_{\widetilde{\mathcal{D} e \ell}}^{(j)} & :=\left(\widetilde{\mathrm{R}}_{j} \cos \widetilde{\mathrm{g}}_{j}-\frac{\widetilde{\Gamma}_{j}}{\widetilde{\mathrm{r}}_{j}} \sin \widetilde{\mathrm{g}}_{j}\right) k^{(1)}+\left(\widetilde{\mathrm{R}}_{j} \sin \widetilde{\mathrm{g}}_{j}+\frac{\widetilde{\Gamma}_{j}}{\widetilde{\mathrm{r}}_{j}} \cos \widetilde{\mathrm{g}}_{j}\right) k^{(2)} .
\end{aligned}
$$

LEMma 2.4 (Delaunay). $\widetilde{\mathcal{D} e l}$ is a bijection from the domain $\mathcal{D}_{\widetilde{\mathcal{D} e l}}$ onto the set

$$
\begin{aligned}
& \mathrm{C}_{\widetilde{\mathcal{D} e \ell}}:=\left\{(y, x)=\left(y^{(1)}, \cdots, y^{(n)}, x^{(1)}, \cdots, x^{(n)}\right) \in \mathbb{R}^{3 n} \times \mathbb{R}^{3 n}:\right. \\
&\left.\widetilde{\mathfrak{n}}_{j}:=k^{(3)} \times \mathrm{C}_{\mathcal{C}}^{(j)} \neq 0, \quad x^{(j)} \neq 0 \quad \forall j=1, \cdots, n\right\}
\end{aligned}
$$

where $\mathrm{C}_{\mathcal{C}}^{(j)}$ is as in (2.23). The formulae for the inverse map

$$
\widetilde{\mathcal{D} e l}^{-1}: \quad(y, x) \in \mathcal{C}_{\widetilde{\mathcal{D} e \ell}} \rightarrow(\widetilde{\mathrm{H}}, \widetilde{\Gamma}, \widetilde{\mathrm{R}}, \widetilde{\mathrm{h}}, \widetilde{\mathrm{g}}, \widetilde{\mathrm{r}}) \in \mathcal{D}_{\widetilde{\mathcal{D} e \ell}}
$$

are

$$
\left\{\begin{array} { l } 
{ \widetilde { \mathrm { H } } _ { j } = \mathrm { C } _ { \mathcal { C } } ^ { ( j ) } \cdot k ^ { ( 3 ) } } \\
{ \widetilde { \mathrm { h } } _ { j } : = \alpha _ { k ^ { ( 3 ) } } ( k ^ { ( 1 ) } , \widetilde { \mathfrak { n } } _ { j } ) }
\end{array} \quad \left\{\begin{array} { l } 
{ \widetilde { \Gamma } _ { j } : = \| \mathrm { C } _ { \mathcal { C } } ^ { ( j ) } \| } \\
{ \widetilde { \mathrm { g } } _ { j } : = \alpha _ { \mathrm { C } _ { \mathcal { C } } ^ { ( j ) } } ( \widetilde { \mathfrak { n } } _ { j } , x ^ { ( j ) } ) }
\end{array} \quad \left\{\begin{array}{l}
\widetilde{\mathrm{R}}_{j}=\frac{y^{(j)} \cdot x^{(j)}}{\left\|x^{(j)}\right\|} \\
\widetilde{\mathrm{r}}_{j}=\left\|x^{(j)}\right\|
\end{array}\right.\right.\right.
$$

Finally, $\widetilde{\mathcal{D} e l}$ preserves the standard Liouville 1-form

$$
\sum_{i=1}^{n} y \frac{(i)}{\mathcal{D} e \ell} \cdot d x \frac{(i)}{\mathcal{D} e \ell}=\sum_{i=1}^{n}\left(\widetilde{\mathrm{H}}_{i} d \widetilde{\mathrm{h}}_{i}+\widetilde{\Gamma}_{i} d \widetilde{\mathrm{g}}_{i}+\widetilde{\mathrm{R}}_{i} d \widetilde{\mathrm{r}}_{i}\right) .
$$

We omit the proof of Lemma 2.4, which may be found in classical textbooks. 
The map $\mathcal{D} e l$.

Definition 2.5 (Delaunay map). Let

$$
\begin{aligned}
& \mathfrak{X}_{\mathcal{D} e \ell}:=\left\{\mathrm{X}_{\mathcal{D} e \ell}:=(\mathrm{H}, \Gamma, \Lambda, \mathrm{h}, \mathrm{g})=\left(\mathrm{H}_{1}, \cdots, \mathrm{H}_{n}, \Gamma_{1}, \cdots, \Gamma_{n}, \Lambda_{1}, \cdots, \Lambda_{n}, \mathrm{~h}_{1}, \cdots, \mathrm{h}_{n},\right.\right. \\
&\left.\mathrm{g}_{1}, \cdots, \mathrm{g}_{n}\right) \in \mathbb{R}^{3 n} \times \mathbb{T}^{2 n}: \quad \Gamma_{j}>0, \quad \frac{\left|\mathrm{H}_{j}\right|}{\Gamma_{j}}<1, \quad \Lambda_{j}>0 \\
&\forall j=1, \cdots, n\}
\end{aligned}
$$

and let $\mathcal{E}_{\mathcal{D} e \ell}$ be the set of n-plets $\left(\mathfrak{E}_{1}, \cdots, \mathfrak{E}_{n}\right)$ where $\mathfrak{E}_{j}=\left(a_{j}, e_{j}, N^{(j)}, P^{(j)}\right)$ satisfies

$$
0<e_{j}<1, \quad \mathfrak{n}_{j}:=k^{(3)} \times N^{(j)} \neq 0, \quad \forall j=1, \cdots, n .
$$

Fix positive numbers $\mathfrak{M}_{1}, \cdots, \mathfrak{M}_{n}, \mathfrak{m}_{1}, \cdots, \mathfrak{m}_{n}$. Define

$$
\tau_{\mathcal{D} e \ell}: \mathrm{X}_{\mathcal{D} e \ell}:=(\mathrm{H}, \Gamma, \Lambda, \mathrm{h}, \mathrm{g}) \in \mathfrak{X}_{\mathcal{D} e \ell} \rightarrow \mathfrak{E}_{\mathcal{D} e \ell}=\left(\mathfrak{E}_{1, \mathcal{D} e \ell}, \cdots, \mathfrak{E}_{n, \mathcal{D} e \ell}\right)
$$

with $\mathfrak{E}_{j, \mathcal{D} e \ell}=\left(a_{j, \mathcal{D} e \ell}, e_{j, \mathcal{D} e \ell}, N_{\mathcal{D} e \ell}^{(j)}, P_{\mathcal{D} e \ell}^{(j)}\right)$ and

$$
\begin{array}{ll}
a_{j, \mathcal{D} e \ell}=\frac{1}{\mathfrak{M}_{j}}\left(\frac{\Lambda_{j}}{\mathfrak{m}_{j}}\right)^{2}, & e_{j, \mathcal{D} e \ell}=\sqrt{1-\left(\frac{\Gamma_{j}}{\Lambda_{j}}\right)^{2}} \\
N_{\mathcal{D} e \ell}^{(j)}=\mathrm{R}_{3}\left(\mathrm{~h}_{\mathrm{j}}\right) \mathcal{R}_{1}\left(i_{j}\right) k^{(3)} & P_{\mathcal{D} e \ell}^{(j)}=\mathrm{R}_{3}\left(\mathrm{~h}_{\mathrm{j}}\right) \mathcal{R}_{1}\left(i_{j}\right) \mathcal{R}_{3}\left(\mathrm{~g}_{j}\right) k^{(1)}
\end{array}
$$

where $i_{j}:=\cos ^{-1} \frac{\mathrm{H}_{j}}{\Gamma_{j}}$.

We call Delaunay map the map

$(2.30)$ Del $: \quad$ Del $=(\mathrm{H}, \Gamma, \Lambda, \mathrm{h}, \mathrm{g}, \ell) \in \mathcal{D}_{\mathcal{D} e \ell} \rightarrow\left(y_{\mathcal{D} e \ell}, x_{\mathcal{D} e \ell}\right) \in \mathbb{R}^{3 n} \times \mathbb{R}^{3 n}$

which is defined on the domain

$$
\mathcal{D}_{\mathcal{D} e \ell}:=\mathfrak{X}_{\mathcal{D} e \ell} \times \mathbb{T}^{n}
$$

as the Kepler map associated to $\tau_{\mathcal{D} e \ell}$ via the following lemma (the proof of which may be found in classical textbooks).

Lemma 2.5 (Delaunay). $\tau_{\mathcal{D} e \ell}$ is a bijection of $\mathfrak{X}_{\mathcal{D} e \ell}$ onto $\mathcal{E}_{\mathcal{D} e \ell}$. Its inverse map

$$
\tau_{\mathcal{D} e \ell}^{-1}: \quad \mathfrak{E}_{\mathcal{D} e \ell}=\left(\mathfrak{E}_{1, \mathcal{D} e \ell}, \cdots, \mathfrak{E}_{n, \mathcal{D} e \ell}\right) \in \mathcal{E}_{\mathcal{D} e \ell} \rightarrow \mathrm{X}_{\mathcal{D} e \ell} \in \mathfrak{X}_{\mathcal{D} e \ell}
$$

is defined by equations

$$
\left\{\begin{array} { l } 
{ \mathrm { H } _ { j } = \mathrm { C } _ { \mathcal { E } } ^ { ( j ) } \cdot k ^ { ( 3 ) } } \\
{ \mathrm { h } _ { j } : = \alpha _ { k ^ { ( 3 ) } } ( k ^ { ( 1 ) } , \mathfrak { n } _ { j } ) }
\end{array} \quad \left\{\begin{array}{l}
\Gamma_{j}=\left\|\mathrm{C}_{\mathcal{E}}^{(j)}\right\| \\
\mathrm{g}_{j}:=\alpha_{\mathrm{C}_{\mathcal{E}}^{(j)}}\left(\mathfrak{n}_{j}, P^{(j)}\right)
\end{array} \quad \Lambda_{j}=\mathfrak{m}_{j} \sqrt{\mathfrak{M}_{j} a_{j}}\right.\right.
$$

where $\mathrm{C}_{\mathcal{E}}^{(j)}$ is as in (2.9). Furthermore, Del preserves the standard 2-form

$$
\sum_{j=1}^{n} d y_{\mathcal{D} e \ell}^{(j)} \wedge d x_{\mathcal{D} e \ell}^{(j)}=\sum_{j=1}^{n}\left(d \mathrm{H}_{j} \wedge d \mathrm{~h}_{j}+d \Gamma_{j} \wedge d \mathrm{~g}_{j}+d \Lambda_{j} \wedge d \ell_{j}\right)
$$

Now we are ready to complete the proof of Theorem 2.1.

Proof of TheOREM 2.1, Let

$$
\mathcal{D}_{\mathcal{P}}^{*}:=\left\{\mathrm{P}=(\Theta, \chi, \Lambda, \vartheta, \kappa, \ell) \in \mathcal{D}_{\mathcal{P}}: \mathrm{P}(\mathrm{P}) \in \mathcal{C}_{\mathcal{D} e \ell}\right\}
$$


It is enough to prove Theorem 2.1 on $\mathcal{D}_{\mathcal{P}}^{*}$, since indeed the $\mathcal{P}$-map is regular on $\mathcal{D}_{\mathcal{P}}=\overline{\mathcal{D}_{\mathcal{P}}^{*}}$. On $\mathcal{D}_{\mathcal{P}}^{*}$, we consider the map

$$
\begin{aligned}
& \phi_{\mathcal{D} e \ell}^{\mathcal{P}}:=\mathcal{D}_{e} \ell^{-1} \circ \mathcal{P}: \\
& \mathrm{P}=(\Theta, \chi, \Lambda, \vartheta, \kappa, \ell) \in \mathcal{D}_{\mathcal{P}}^{*} \rightarrow \operatorname{Del}=(\mathrm{H}, \Gamma, \Lambda, \mathrm{h}, \mathrm{g}, \ell) \in \mathcal{D}_{\mathcal{D} e \ell}^{*}:=\phi_{\mathcal{D} e \ell}^{\mathcal{P}}\left(\mathcal{D}_{\mathcal{P}}^{*}\right) \subset \mathcal{D}_{\mathcal{D} e \ell} .
\end{aligned}
$$

$\phi_{\mathcal{D} e \ell}^{\mathcal{P}}$ gives the Delaunay coordinates at left hand side in (2.30) in terms of the P-coordinates at left hand side of (2.7) in the subset $\mathcal{D}_{\mathcal{P}}^{*}$ of $\mathcal{D}_{\mathcal{P}}$ the $\mathcal{P}$-image of which lies in the $\mathcal{D} e \ell$-image of $\mathcal{D}_{\mathcal{D} e \ell}$. Clearly, $\phi_{\mathcal{D} e \ell}^{\mathcal{P}}$ leaves the $(\Lambda, \ell)$ unvaried. More precisely, $\phi_{\mathcal{D} e \ell}^{\mathcal{P}}$ decouples into two disjoint maps: the identity on the $(\Lambda, \ell)$, and a $4 n$-dimensional map

$$
\widehat{\phi_{\mathcal{D} e \ell}^{\mathcal{P}}}: \quad(\Theta, \chi, \vartheta, \kappa) \in \widehat{\mathcal{D}_{\mathcal{P}}^{*}} \rightarrow(\mathrm{H}, \Gamma, \mathrm{h}, \mathrm{g}) \in \widehat{\mathcal{D}_{\mathcal{D} e \ell}^{*}}=\phi_{\mathcal{D} e \ell}^{\mathcal{P}}\left(\widehat{\mathcal{D}_{\mathcal{P}}^{*}}\right) \subset \widehat{\mathcal{D}_{\mathcal{D} e \ell}}
$$

on the remaining coordinates, which turns out to be a bijection of the sets $\widehat{\mathcal{D}_{\mathcal{P}}^{*}}$ and $\widehat{\mathcal{D}_{\mathcal{D} e \ell}^{*}}$. Here, the map $\widehat{\phi_{\mathcal{D} e \ell}^{\mathcal{P}}}$ and the sets $\widehat{\mathcal{D}_{\mathcal{P}}^{*}}$ and $\widehat{\mathcal{D}_{\mathcal{D} e \ell}}$ do not depend on $(\Lambda, \ell)$. Indeed, the explicit expressions of $\widehat{\phi_{\mathcal{D} e \ell}}, \widehat{\mathcal{D}_{\mathcal{P}}^{*}}$ in terms of $\mathrm{P}=(\Theta, \chi, \Lambda, \vartheta, \kappa, \ell)$; or of $\widehat{\mathcal{D}_{\mathcal{D} e \ell}}$ in terms of Del $=(\mathrm{H}, \Gamma, \Lambda, \mathrm{h}, \mathrm{g}, \ell)$ involve only the $\mathrm{C}_{\mathcal{P}}^{(j)}, P_{\mathcal{P}}^{(j)}$; the $\mathrm{C}_{\mathcal{D} e \ell}^{(j)}, P_{\mathcal{D} e \ell}^{(j)}$, that do not depend on $(\Lambda, \ell)$ : (2.31) (where one has to replace $\mathcal{C}$ with $\mathcal{P}$ ), (2.15) and (2.18).

In view of the previous consideration and of Lemma 2.5, Theorem 2.1] is implied by

LEMMA 2.6. The map $\widehat{\phi_{\mathcal{D} e \ell}^{\mathcal{P}}}$ preserves that standard 1-form:

$$
\sum_{j=1}^{n}\left(\mathrm{H}_{j} d \mathrm{~h}_{j}+\Gamma_{j} d \mathrm{~g}_{j}\right)=\sum_{j=1}^{n}\left(\Theta_{j-1} d \vartheta_{j-1}+\chi_{j-1} d \kappa_{j-1}\right) .
$$

Proof. We look at the analogous map

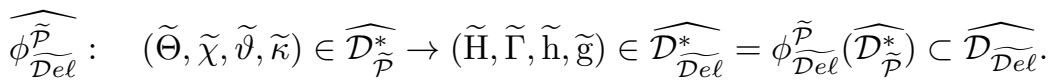

The analytical expression of this map is identical to the one of $\widehat{\phi_{\mathcal{D} e \ell}^{\mathcal{P}}}$. This follows from the fact that $\widehat{\widehat{\widehat{\mathcal{P}}}}$ depends on the coordinates $(\widetilde{\Theta}, \widetilde{\chi}, \widetilde{\vartheta}, \widetilde{\kappa})$ only via $\mathrm{C}_{\widetilde{\mathcal{P}}}^{(j)}$ and $\frac{x_{\tilde{\mathcal{P}}}^{(j)}}{\left\|x_{\tilde{\mathcal{P}}}^{(j)}\right\|}$ exactly as $\widehat{\phi_{\mathcal{D} e \ell}^{\mathcal{P}}}$ depends on $(\Theta, \chi, \vartheta, \kappa)$ only via $\mathrm{C}_{\mathcal{P}}^{(j)}$ and $\Pi_{\mathcal{P}}^{(j)}$, that $\mathrm{C}_{\widetilde{\mathcal{P}}}^{(j)}$ and $\frac{x_{\tilde{\mathcal{P}}}^{(j)}}{\left\|x_{\tilde{\mathcal{P}}}^{(j)}\right\|}$ have exactly the same expressions of $\mathrm{C}_{\mathcal{P}}^{(j)}$ and $P^{(j)}$, apart for replacing $(\Theta, \chi, \vartheta, \kappa)$ with $(\widetilde{\Theta}, \widetilde{\chi}, \widetilde{\vartheta}, \widetilde{\kappa})$. Compare (2.29) (where one has to replace $\mathrm{C}_{\mathcal{C}}^{(j)}$ with $\mathrm{C}_{\widetilde{\mathcal{P}}}^{(j)}$ ), (2.31) (where one has to replace $\mathrm{C}_{\mathcal{E}}^{(j)}$ with $\mathrm{C}_{\mathcal{P}}^{(j)}$ ), (2.15), (2.18), (2.24) and (2.25). But Lemmata 2.2 and 2.4 imply that $\widehat{\phi_{\widetilde{\mathcal{D}}}^{\widetilde{\mathcal{P}}}}$ preserves that standard 1-form:

$$
\sum_{j=1}^{n}\left(\widetilde{\mathrm{H}}_{j} d \widetilde{\mathrm{h}}_{j}+\widetilde{\Gamma}_{j} d \widetilde{\mathrm{g}}_{j}\right)=\sum_{j=1}^{n}\left(\widetilde{\Theta}_{j-1} d \widetilde{\vartheta}_{j-1}+\widetilde{\chi}_{j-1} d \widetilde{\kappa}_{j-1}\right)
$$

Then $\widehat{\phi_{\mathcal{D} e \ell}^{\mathcal{P}}}$ does. 


\subsection{The $\mathcal{P}$-map vs rotations and reflections}

Now we discuss how the $\mathcal{P}$-map behaves in presence of symmetries in the Hamiltonian due to rotations or reflections.

Let $\mathrm{H}=\mathrm{H}(y, x)$ be the Hamiltonian governing the motion of $n$ particles, where such particles are expressed in the canonical coordinates $\left(y^{(1)}, x^{(1)}\right), \cdots,\left(y^{(n)}, x^{(n)}\right)$. Assume that $\mathrm{H}$ is left unvaried by rotations and reflections. Namely, if

$$
\phi_{\mathcal{R}, \mathcal{S}}: \quad\left(y^{(j)}, x^{(j)}\right) \rightarrow\left(\mathrm{R} y^{(j)}, \mathcal{S} x^{(j)}\right), \quad j=1, \cdots, n
$$

where $\mathcal{R}, \mathcal{S}$ are real a $3 \times 3$ matrices, then rotation invariance is

$$
\mathrm{H} \circ \phi_{\mathcal{R}, \mathcal{R}}=\mathrm{H} \quad \forall \mathcal{R}: \mathcal{R}^{\mathrm{t}}=\mathrm{id}
$$

while reflection invariance is

$$
\begin{aligned}
& \mathrm{H} \circ \phi_{\mathcal{S}_{\sigma}, \mathcal{S}_{\tau}}=\mathrm{H} \quad \text { for some } \mathcal{S}_{\sigma}=\left(\begin{array}{lll}
\sigma_{1} & 0 & 0 \\
0 & \sigma_{2} & 0 \\
0 & 0 & \sigma_{3}
\end{array}\right) \quad \mathcal{S}_{\tau}=\left(\begin{array}{lll}
\tau_{1} & 0 & 0 \\
0 & \tau_{2} & 0 \\
0 & 0 & \tau_{3}
\end{array}\right) \\
& \sigma_{i}, \tau_{i}= \pm 1 .
\end{aligned}
$$

Rotation invariance is associated to the conservation, through the motion, of the total angular momentum $\mathrm{S}_{\mathcal{C}}^{(1)}$ is (2.23). Reflection invariance is not associated to integrals.

The Hamiltonian $\mathrm{H}_{\text {hel }}$ in (3.33) is rotation and reflection invariant, and reflection invariance holds with any choice of $\sigma, \tau$.

Let

$$
\mathrm{H}_{\mathcal{P}}:=\mathrm{H} \circ \mathcal{P} .
$$

The fact that $\mathrm{S}_{\mathcal{C}}^{(1)}$ is preserved along the motions of $\mathrm{H}$ implies that the coordinates

$$
\Theta_{0}=Z, \quad \vartheta_{0}=\zeta, \quad \kappa_{0}=\mathfrak{g}
$$

do not appear in $\mathrm{H}_{\mathcal{P}}$. Indeed, $Z$ and $\zeta$ are integrals, while $\mathfrak{g}$ is conjugated to $\mathrm{G}=\left\|\mathrm{S}_{\mathcal{P}}^{(1)}\right\|$, which is an integral for $\mathrm{H}_{\mathcal{P}}$. Thus, the number of degrees of freedom is naturally reduced by two units, once one regards $\mathrm{G}$ as a prefixed external parameter. Namely, for any fixed $\chi_{0}=\mathrm{G}, \mathrm{H}_{\mathcal{K}}$ may be regarded as a function of the $2(3 n-1)$ dimensional coordinates

$$
\overline{\mathrm{P}}:=(\bar{\Theta}, \chi, \Lambda, \bar{\vartheta}, \kappa, \ell)
$$

which does not depend on $\kappa_{0}$. Here,

$$
\bar{\Theta}=\left(\Theta_{1}, \cdots, \Theta_{n-1}\right), \quad \bar{\vartheta}=\left(\vartheta_{1}, \cdots, \vartheta_{n-1}\right) .
$$

An analogue property is also shared with the action-angle coordinates $(\Psi, \Gamma, \Lambda$, $\psi, \gamma, \ell)$ described in [7, 27, and related to a set of coordinates discovered by $\mathrm{A}$. Deprit [12 in the 80s (compare also [36] or the Appendix E).

The main novelty introduced by the $\mathcal{P}$-coordinates (that does not hold for the coordinates of [7]) is how $\mathcal{P}$ behaves relative to reflections.

We denote as

$$
\mathcal{R}_{2}^{-}:=\phi_{\mathcal{S}_{\sigma(2)}, \mathcal{S}_{\sigma(2)}} \quad \sigma^{(2)}=(1,-1,1)
$$

the reflection of the second coordinate both for the $y^{(j)}$ 's and the $x^{(j)}$ 's and we let

$$
\mathcal{S}^{-}(\Theta, \chi, \Lambda, \vartheta, \kappa, \ell):=(-\Theta, \chi, \Lambda,-\vartheta, \kappa, \ell) .
$$


Proposition 2.2.

$$
\mathrm{R}_{2}^{-} \circ \mathcal{P}=\mathcal{P} \circ \mathcal{S}^{-}
$$

Therefore, if $\mathrm{H}=\mathrm{H}(y, x)$ satisfies

$$
\mathrm{H} \circ \mathcal{R}_{2}^{-}=\mathrm{H}
$$

then $\mathrm{H}_{\mathcal{P}}:=\mathrm{H} \circ \mathcal{P}$ satisfies

$$
\mathrm{H}_{\mathcal{P}} \circ \mathcal{S}^{-}=\mathrm{H}_{\mathcal{P}}
$$

Hence, any of the points

$$
\Theta_{0}=\cdots=\Theta_{n-1}=0, \quad\left(\vartheta_{0}, \cdots, \vartheta_{n-1}\right)=\left(k_{0}, \cdots, k_{n-1}\right) \pi \quad \bmod 2 \pi \mathbb{Z}^{n}
$$

is an equilibrium point for $\mathrm{H}_{\mathcal{P}}$, for any $(\chi, \Lambda, \kappa, \ell)$.

Proof. Defining $\mathcal{R}^{(j)}:=\mathrm{T}_{j} \mathrm{~S}_{j}, s^{(j)}:=\mathrm{T}_{j} k^{(3)}$, we write the vectors $P_{\mathcal{P}}^{(j)}$ and $\mathrm{S}_{\mathcal{P}}^{(j)}$ (compare Eq. (2.18) and Remark 2.2(iii)) as

$$
P_{\mathcal{P}}^{(j)}=\mathcal{R}^{(1)} \cdots \mathcal{R}^{(j)} k^{(3)}, \quad \mathrm{S}_{\mathcal{P}}^{(j)}=\chi_{j-1} \mathcal{R}^{(1)} \cdots \mathcal{R}^{(j)} s^{(j)} .
$$

The explicit expressions of $\mathcal{R}^{(j)}$ and $s^{(j)}$ are

$$
\begin{aligned}
\mathcal{R}_{11}^{(j)} & =\cos \kappa_{j-1} \cos \vartheta_{j-1}-\sin \kappa_{j-1} \cos \iota_{j} \sin \vartheta_{j-1} \\
\mathcal{R}_{21}^{(j)} & =\cos \kappa_{j-1} \sin \vartheta_{j-1}+\sin \kappa_{j-1} \cos \iota_{j} \cos \vartheta_{j-1} \\
\mathcal{R}_{31}^{(j)} & =\sin \kappa_{j-1} \sin \iota_{j} \\
\mathcal{R}_{12}^{(j)} & =-\cos i_{j} \sin \kappa_{j-1} \cos \vartheta_{j-1}+\sin \vartheta_{j-1}\left(-\cos i_{j} \cos \iota_{j} \cos \kappa_{j-1}+\sin \iota_{j} \sin \mathrm{i}_{\mathrm{j}}\right) \\
\mathcal{R}_{22}^{(j)} & =-\cos \mathrm{i}_{j} \sin \kappa_{j-1} \sin \vartheta_{j-1}-\cos \vartheta_{j-1}\left(-\cos \mathrm{i}_{j} \cos \iota_{j} \cos \kappa_{j-1}+\sin \iota_{j} \sin \mathrm{i}_{\mathrm{j}}\right) \\
\mathcal{R}_{32}^{(j)} & =\cos i_{j} \cos \kappa_{j-1} \sin \iota_{j}+\sin \mathrm{i}_{j} \cos \iota_{j} \\
\mathcal{R}_{13}^{(j)} & =\sin \mathrm{i}_{j} \sin \kappa_{j-1} \cos \vartheta_{j-1}+\sin \vartheta_{j-1}\left(\sin \mathrm{i}_{j} \cos \iota_{j} \cos \kappa_{j-1}+\sin \iota_{j} \cos \mathrm{i}_{\mathrm{j}}\right) \\
\mathcal{R}_{23}^{(j)} & =\sin \mathrm{i}_{j} \sin \kappa_{j-1} \sin \vartheta_{j-1}-\cos \vartheta_{j-1}\left(\sin \mathrm{i}_{j} \cos \iota_{j} \cos \kappa_{j-1}+\sin \iota_{j} \cos \mathrm{i}_{\mathrm{j}}\right) \\
\mathcal{R}_{33}^{(j)} & =-\sin \mathrm{i}_{j} \cos \kappa_{j-1} \sin \iota_{j}+\cos \mathrm{i}_{j} \cos \iota_{j} \\
s_{1}^{(j)} & =\sin \iota_{j} \sin \vartheta_{j-1} \\
s_{2}^{(j)} & =-\sin \iota_{j} \cos \vartheta_{j-1} \\
s_{3}^{(j)} & =\cos \iota_{j} .
\end{aligned}
$$

Then $\mathcal{S}^{-}$lets $P_{\mathcal{P}}^{(j)}$ and $\mathrm{S}_{\mathcal{P}}^{(j)}$ respectively, into

$$
\left(P_{\mathcal{P}}^{(j)}\right)^{-}:=\mathcal{R}_{2}^{-} P_{\mathcal{P}}^{(j)} \quad \text { and } \quad\left(\mathrm{S}_{\mathcal{P}}^{(j)}\right)^{-}:=-\mathcal{R}_{2}^{-} \mathrm{S}_{\mathcal{P}}^{(j)} .
$$

Therefore, $\mathrm{C}_{\mathcal{P}}^{(j)}=\mathrm{S}_{\mathcal{P}}^{(j)}-\mathrm{S}_{\mathcal{P}}^{(j+1)}\left(\right.$ with $\left.\mathrm{S}_{\mathcal{P}}^{(n+1)}:=0\right)$ and $Q_{\mathcal{P}}^{(j)}=\frac{\mathrm{C}_{\mathcal{P}}^{(j)}}{\left\|\mathrm{C}_{\mathcal{P}}^{(j)}\right\|} \times P_{\mathcal{P}}^{(j)}$ are transformed, respectively, into

$$
\left(\mathrm{C}_{\mathcal{P}}^{(j)}\right)^{-}:=-\mathcal{R}_{2}^{-} \mathrm{C}_{\mathcal{P}}^{(j)}, \quad\left(Q_{\mathcal{P}}^{(j)}\right)^{-}:=\mathcal{R}_{2}^{-} Q_{\mathcal{P}}^{(j)} .
$$

On the other hand, $a_{j, \mathcal{P}}$ and $e_{j, \mathcal{P}}$ are left unvaried by $\mathcal{S}^{-}$. In view of Definition 2.2 and Definition 2.3. the thesis (2.32) follows. 
This is a free offprint provided to the author by the publisher. Copyright restrictions may apply. 


\section{CHAPTER 3}

\section{The $\mathcal{P}$-map and the planetary problem}

After the reduction of the invariance by translations, a Hamiltonian governing the motions of $n$ planets with masses $\mu m_{1}, \cdots, \mu m_{n}$ interacting among themselves and with a star with mass $m_{0}$ can be taken to be the "heliocentric" one

$$
\mathrm{H}_{\text {hel }}:=\sum_{1 \leq i \leq n}\left(\frac{\left\|y^{(i)}\right\|^{2}}{2 \mathfrak{m}_{i}}-\frac{\mathfrak{m}_{i} \mathfrak{M}_{i}}{\left\|x^{(i)}\right\|}\right)+\mu \sum_{1 \leq i<j \leq n}\left(\frac{y^{(i)} \cdot y^{(j)}}{m_{0}}-\frac{m_{i} m_{j}}{\left\|x^{(i)}-x^{(j)}\right\|}\right)
$$

where $(y, x)=\left(y^{(1)}, \cdots, y^{(n)}, x^{(1)}, \cdots, x^{(n)}\right)$ are "Cartesian coordinates" taking values on the "collision-less" phase space $\mathbb{R}^{3 n} \times \mathbb{R}^{3 n} \backslash \Delta$, where

$$
\Delta=\left\{x=\left(x^{(1)}, \cdots, x^{(n)}\right) \in \mathbb{R}^{3} \times \cdots \times \mathbb{R}^{3}: \quad 0 \neq x^{(i)} \neq x^{(j)} \quad \forall 1 \leq i<j \leq n\right\}
$$

endowed with the standard 2- form

$$
\Omega:=d y \wedge d x:=\sum_{i=1}^{n} \sum_{j=1}^{3} d y_{j}^{(i)} \wedge d x_{j}^{(i)}
$$

and with

$$
\mathfrak{M}_{i}=m_{0}+\mu m_{i} \quad \mathfrak{m}_{i}=\frac{m_{0} m_{i}}{m_{0}+\mu m_{i}}
$$

being the so-called "reduced masses".

In the following Chapter 3.1 we describe a general property of Kepler maps, in relation to their application to the Hamiltonian $\mathrm{H}_{\text {hel }}$. Then (in Chapter 3.2) we shall specialize to the case of the $\mathcal{P}$-map.

\subsection{A general property of Kepler maps}

For a general Kepler map $\mathcal{K}$, we denote

$$
\mathrm{H}_{\mathcal{K}}(\mathrm{K}):=\mathrm{H}_{\text {hel }} \circ \mathcal{K}=-\sum_{j=1}^{n} \frac{\mathfrak{m}_{j} \mathfrak{M}_{j}}{2 a_{j, \mathcal{K}}\left(\mathrm{X}_{\mathcal{K}}\right)}+\mu f_{\mathcal{K}}(\mathrm{K})
$$

where

$$
f_{\mathcal{K}}(\mathrm{K}):=\sum_{1 \leq i<j \leq n}\left(\frac{y_{\mathcal{K}}^{(i)} \cdot y_{\mathcal{K}}^{(j)}}{m_{0}}-\frac{m_{i} m_{j}}{\left\|x_{\mathcal{K}}^{(i)}-x_{\mathcal{K}}^{(j)}\right\|}\right)
$$

and $y_{\mathcal{K}}^{(j)}, x_{\mathcal{K}}^{(j)}$ are as in Definition 2.2 .

We denote

$$
\overline{f_{\mathcal{K}}}\left(\mathrm{X}_{\mathcal{K}}\right):=\frac{1}{(2 \pi)^{n}} \int_{\mathbb{T}^{n}} f_{\mathcal{K}}\left(\mathrm{X}_{\mathcal{K}}, \ell\right) d \ell
$$


so that

$$
\begin{array}{ll}
f_{\mathcal{K}}=\sum_{1 \leq i<j \leq n} f_{\mathcal{K}}^{i j}, & \overline{f_{\mathcal{K}}}=\sum_{1 \leq i<j \leq n} \overline{f_{\mathcal{K}}^{i j}} \\
f_{\mathcal{K}}^{i j}:=\frac{y_{\mathcal{K}}^{(i)} \cdot y_{\mathcal{K}}^{(j)}}{m_{0}}-\frac{m_{i} m_{j}}{\left\|x_{\mathcal{K}}^{(i)}-x_{\mathcal{K}}^{(j)}\right\|}, \quad \overline{f_{\mathcal{K}}^{i j}}:=\frac{1}{(2 \pi)^{n}} \int_{\mathbb{T}^{n}} f_{\mathcal{K}}^{i j} d \ell_{1} \cdots d \ell_{n} .
\end{array}
$$

For a general Kepler map, one always has, as a consequence of (2.6),

$$
\begin{array}{rlrl}
-\frac{1}{2 \pi} \int_{\mathbb{T}} T_{\mathcal{K}}^{(j)} d \ell_{j} & =\frac{1}{2 \pi} \int_{\mathbb{T}} \frac{V_{\mathcal{K}}^{(j)}}{2} d \ell_{j}=T_{\mathcal{K}}^{(j)}+V_{\mathcal{K}}^{(j)}=-\frac{\mathfrak{m M}}{2 a_{j, \mathcal{K}}} \\
\frac{1}{2 \pi} \int_{\mathbb{T}} y_{\mathcal{K}}^{(j)} d \ell_{j} & =0 & \frac{1}{2 \pi} \int_{\mathbb{T}} \frac{x_{\mathcal{K}}^{(j)}}{\left\|x_{\mathcal{K}}^{(j)}\right\|^{3}} d \ell_{j}=0,
\end{array}
$$

where we have denoted as

$$
T_{\mathcal{K}}^{(j)}:=\frac{\left\|y_{\mathcal{K}}^{(j)}\right\|^{2}}{2 \mathfrak{m}_{j}} \quad V_{\mathcal{K}}^{(j)}:=-\frac{\mathfrak{m}_{j} \mathfrak{M}_{j}}{\left\|x_{\mathcal{K}}^{(j)}\right\|}
$$

the kinetic, potential part of $\mathrm{H}_{\mathcal{K}}^{(j)}$ in (2.4), respectively.

Consider the average $\overline{f_{\mathcal{K}}}\left(\mathrm{X}_{\mathcal{K}}\right)$ in 3.35 . Due to the fact that $y_{\mathcal{K}}^{(j)}$ has zero-average, one has that only the Newtonian part contributes to $\overline{f_{\mathcal{K}}}\left(\mathrm{X}_{\mathcal{K}}\right)$ :

$$
\overline{f_{\mathcal{K}}}=-\sum_{1 \leq i<j \leq n} \frac{m_{i} m_{j}}{(2 \pi)^{2}} \int_{\mathbb{T}^{2}} \frac{d \ell_{i} d \ell_{j}}{\left\|x_{\mathcal{K}}^{(i)}-x_{\mathcal{K}}^{(j)}\right\|} .
$$

We now consider any of the contributions to this sum

$$
\overline{f_{\mathcal{K}}^{i j}}=-\frac{m_{i} m_{j}}{(2 \pi)^{2}} \int_{\mathbb{T}^{2}} \frac{d \ell_{i} d \ell_{j}}{\left\|x_{\mathcal{K}}^{(i)}-x_{\mathcal{K}}^{(j)}\right\|} \quad 1 \leq i<j \leq n
$$

and expand any such terms

$$
\overline{f_{\mathcal{K}}^{i j}}={\overline{f_{\mathcal{K}}^{i j}}}^{(0)}+{\overline{f_{\mathcal{K}}^{i j}}}^{(1)}+{\overline{f_{\mathcal{K}}^{i j}}}^{(2)}+\cdots
$$

where

$$
{\overline{f_{\mathcal{K}}^{i j}}}^{(h)}:=-\left.\frac{m_{i} m_{j}}{(2 \pi)^{2}} \int_{\mathbb{T}^{2}} \frac{1}{h !} \frac{d^{h}}{d \varepsilon^{h}} \frac{1}{\left\|\varepsilon x_{\mathcal{K}}^{(i)}-x_{\mathcal{K}}^{(j)}\right\|}\right|_{\varepsilon=0} d \ell_{i} d \ell_{j}
$$

is proportional to $\frac{1}{a_{j}}\left(\frac{a_{i}}{a_{j}}\right)^{h}$. Then the formulae in (3.36) imply that the two first terms of this expansion are given by

$$
{\overline{f_{\mathcal{K}}^{i j}}}^{(0)}=-\frac{m_{i} m_{j}}{a_{j, \mathcal{K}}}, \quad{\overline{f_{\mathcal{K}}^{i j}}}^{(1)}=0 .
$$

Namely, whatever is the Kepler map that is used, the first term that depends on the secular coordinates $\mathrm{X}_{\mathcal{K}}$ is the double average of the second order term

$$
{\overline{f_{\mathcal{K}}^{i j}}}^{(2)}\left(\mathrm{X}_{\mathcal{K}}\right)=-\frac{m_{i} m_{j}}{(2 \pi)^{2}} \int_{\mathbb{T}^{2}} \frac{3\left(x_{\mathcal{K}}^{(i)} \cdot x_{\mathcal{K}}^{(j)}\right)^{2}-\left\|x_{\mathcal{K}}^{(i)}\right\|^{2}\left\|x_{\mathcal{K}}^{(j)}\right\|^{2}}{\left\|x_{\mathcal{K}}^{(j)}\right\|^{5}} d \ell_{i} d \ell_{j} .
$$

Now we specialize to the case of the $\mathcal{P}$-map. 


\subsection{The case of the $\mathcal{P}$-map}

We denote as

$$
\mathrm{H}_{\mathcal{P}}\left(\mathrm{X}_{\mathcal{P}}, \ell\right)=\mathrm{h}_{\mathfrak{f a s t}}^{0}(\Lambda)+\mu f_{\mathcal{P}}\left(\mathrm{X}_{\mathcal{P}}, \ell\right) \quad \mathrm{X}_{\mathcal{P}}:=(\Theta, \chi, \Lambda, \vartheta, \kappa)
$$

where

$$
\mathrm{h}_{\mathfrak{f a s t}}^{0}(\Lambda):=-\sum_{j=1}^{n} \frac{\mathfrak{m}_{j}^{3} \mathfrak{M}_{j}^{2}}{2 \Lambda_{j}^{2}},
$$

is the Hamiltonian (3.33) expressed in $\mathcal{P}$-coordinates.

Using the definitions, it not difficult to see that

Lemma 3.1. $\overline{f_{\mathcal{P}}^{i j}}, f_{\mathcal{P}}^{i j}$ depend, respectively, only on the coordinates

$$
\begin{aligned}
& \overline{\mathrm{X}_{\mathcal{P}}^{i j}}:=\left(\Theta_{i}, \cdots, \Theta_{j \wedge(n-1)}, \chi_{i-1}, \cdots, \chi_{j \wedge(n-1)}, \Lambda_{i}, \Lambda_{j}, \vartheta_{i}, \cdots, \vartheta_{j \wedge(n-1)}, \kappa_{i}, \cdots, \kappa_{j-1}\right) \\
& \overline{\mathrm{P}^{i j}}:=\left(\overline{\mathrm{X}_{\mathcal{P}}^{i j}}, \ell_{i}, \ell_{j}\right)
\end{aligned}
$$

with $a \wedge b$ denoting the minimum of $a$ and $b$.

Accordingly to the previous lemma, the "nearest-neighbor" terms $\overline{f_{\mathcal{P}}^{i, i+1}}$, with $i=1, \cdots, n-1$, depend only on

$$
\overline{\mathrm{X}_{\mathcal{P}}^{i, i+1}}= \begin{cases}\left(\Theta_{i}, \Theta_{i+1}, \chi_{i-1}, \chi_{i}, \chi_{i+1}, \Lambda_{i}, \Lambda_{i+1}, \vartheta_{i}, \vartheta_{i+1}, \kappa_{i}\right) & 1 \leq i \geq 3 \& \\ \left(\Theta_{n-1}, \chi_{n-2}, \chi_{n-1}, \Lambda_{n-1}, \Lambda_{n}, \vartheta_{n-1}, \kappa_{n-1}\right) & i=n-1 .\end{cases}
$$

However, for the functions ${\overline{f_{\mathcal{P}}^{i, i+1}}}^{(2)}$, we have a special rule. Indeed, for any Kepler map $\mathcal{K}$, the "exterior" angular momentum $\left\|\mathrm{C}_{\mathcal{K}}^{(i+1)}\right\|$ is an integral for ${\overline{f_{\mathcal{K}}^{i, i+1}}}^{(2)}$. This readily implies that any ${\overline{f_{\mathcal{K}}^{i, i+1}}}^{(2)}$ is integrable, for having four degrees of freedom and four independent, commuting integrals $\left(\left\|\mathrm{C}_{\mathcal{K}}^{(i)}+\mathrm{C}_{\mathcal{K}}^{(i+1)}\right\|,\left(\mathrm{C}_{\mathcal{K}}^{(i)}+\mathrm{C}_{\mathcal{K}}^{(i+1)}\right) \cdot k^{(3)}\right.$, $\left\|\mathrm{C}_{\mathcal{K}}^{(i+1)}\right\|$ and ${\overline{f_{\mathcal{K}}^{i, i+1}}}^{(2)}$ itself). This fact has been firstly noticed, in the three-body case $(i=1, n=2)$, by R. Harrington $[19$ who, using the Jacobi reduction of the nodes $\mathcal{J} a c$, where the coordinates are named

$$
\mathrm{G}_{i}, \quad \mathrm{~g}_{i}, \quad \Lambda_{i}, \quad \ell_{i}, \quad i=1,2
$$

(with $\mathrm{G}_{i}=\left\|\mathrm{C}^{(i)}\right\|, \mathrm{g}_{i}$ related to the perihelia directions, and $\mathrm{G}:=\|\mathrm{C}\|, \mathrm{C}=$ $\mathrm{C}^{(1)}+\mathrm{C}^{(2)}$ appearing as an external parameter), noticed that ${\overline{f_{\mathcal{J} a c}^{12}}}^{(2)}$ depends only on $\left(\mathrm{G}, \mathrm{G}_{1}, \mathrm{G}_{2}, \gamma_{1}, \Lambda_{1}, \Lambda_{2}\right)$.

Let us now inspect how the integrability of ${\overline{f_{\mathcal{P}}^{i, i+1}}}^{(2)}$ is exhibited in terms of the $\mathcal{P}$-map. Since $\left\|\mathrm{C}_{\mathcal{P}}^{(n)}\right\|=\chi_{n-1}$, one has that ${f_{\mathcal{P}}^{n-1, n}}^{(2)}$ does not depend on $\kappa_{n-1}$, and hence, by (3.39), depends only on

$$
\overline{\overline{\mathrm{X}_{\mathcal{P}}^{n-1, n}}}:=\left(\Theta_{n-1}, \chi_{n-2}, \chi_{n-1}, \Lambda_{n-1}, \Lambda_{n}, \vartheta_{n-1}\right) .
$$

This fact, for $n \geq 3$, is no longer true for $i=1, \cdots, n-2$, because in that case $\chi_{i} \neq\left\|\mathrm{C}_{\mathcal{P}}^{(i+1)}\right\|$ (indeed, $\left.\chi_{i}=\left\|\mathrm{S}_{\mathcal{P}}^{(i+1)}\right\|\right)$. However, since, for $\left(\Theta_{i+1}, \vartheta_{i+1}\right)=(0, \pi)$, 
$\left\|\mathrm{C}_{\mathcal{P}}^{(i+1)}\right\|$ reduces to

$$
\left.\left\|\mathrm{C}_{\mathcal{P}}^{(i+1)}\right\|\right|_{\left(\Theta_{i+1}, \vartheta_{i+1}\right)=(0, \pi)}=\chi_{i}-\chi_{i+1} \quad i=1, \cdots, n-2,
$$

one has that the functions

$$
{\overline{\overline{f_{\mathcal{P}}^{i, i+1}}}}^{(2)}:=\left.{\overline{f_{\mathcal{P}}^{i, i+1}}}^{(2)}\right|_{\left(\Theta_{i+1}, \vartheta_{i+1}\right)=(0, \pi)}, \quad i=1, \cdots, n-2
$$

do not depend on $\kappa_{i}$ and hence, by (3.39) depend only on

$$
\overline{\overline{\mathrm{X}_{\mathcal{P}}^{i, i+1}}}:=\left(\Theta_{i}, \chi_{i-1}, \chi_{i}, \chi_{i+1}, \Lambda_{i}, \Lambda_{i+1}, \vartheta_{i}\right), \quad i=1, \cdots, n-2 .
$$

In the following lemma we provide their explicit expressions.

Lemma 3.2. The function ${\overline{f_{\mathcal{P}}^{n-1, n}}}^{(2)}$ and, for $n \geq 3$ and $1 \leq i \leq n-2$, the functions ${\overline{\overline{f \mathcal{P}}^{i, i+1}}}^{(2)}$ have the following expressions

$$
\begin{aligned}
\bar{f}_{\mathcal{P}}^{n-1, n}(2)= & m_{n-1} m_{n} \frac{a_{n-1}^{2}}{4 a_{n}^{3}} \frac{\Lambda_{n}^{3}}{\chi_{n-1}^{5}}\left[\frac{5}{2}\left(3 \Theta_{n-1}^{2}-\chi_{n-1}^{2}\right)\right. \\
- & \frac{3}{2} \frac{4 \Theta_{n-1}^{2}-\chi_{n-1}^{2}}{\Lambda_{n-1}^{2}}\left(\chi_{n-2}^{2}+\chi_{n-1}^{2}-2 \Theta_{n-1}^{2}\right. \\
& \left.+2 \sqrt{\left(\chi_{n-1}^{2}-\Theta_{n-1}^{2}\right)\left(\chi_{n-2}^{2}-\Theta_{n-1}^{2}\right)} \cos \vartheta_{n-1}\right) \\
+ & \left.\frac{3}{2} \frac{\left(\chi_{n-1}^{2}-\Theta_{n-1}^{2}\right)\left(\chi_{n-2}^{2}-\Theta_{n-1}^{2}\right)}{\Lambda_{n-1}^{2}} \sin ^{2} \vartheta_{n-1}\right]
\end{aligned}
$$

and

$$
\begin{aligned}
{\overline{f_{\mathcal{P}}^{i, i+1}}}^{(2)} & =m_{i} m_{i+1} \frac{a_{i}^{2}}{4 a_{i+1}^{3}} \frac{\Lambda_{i+1}^{3}}{\chi_{i}^{2}\left(\chi_{i}-\chi_{i+1}\right)^{3}}\left[\frac{5}{2}\left(3 \Theta_{i}^{2}-\chi_{i}^{2}\right)\right. \\
& -\frac{3}{2} \frac{4 \Theta_{i}^{2}-\chi_{i}^{2}}{\Lambda_{i}^{2}}\left(\chi_{i-1}^{2}+\chi_{i}^{2}-2 \Theta_{i}^{2}+2 \sqrt{\left(\chi_{i}^{2}-\Theta_{i}^{2}\right)\left(\chi_{i-1}^{2}-\Theta_{i}^{2}\right)} \cos \vartheta_{i}\right) \\
& \left.+\frac{3}{2} \frac{\left(\chi_{i}^{2}-\Theta_{i}^{2}\right)\left(\chi_{i-1}^{2}-\Theta_{i}^{2}\right)}{\Lambda_{i}^{2}} \sin ^{2} \vartheta_{i}\right] .
\end{aligned}
$$

Lemma 3.2 is proved in Appendix B. Here, we limit to the following.

REMARK 3.3.

(i) The formula in (3.41) holds also for complex values of the coordinates, provided that $\arg \left(\chi_{i}-\chi_{i+1}\right) \in\left(-\frac{\pi}{2}, \frac{\pi}{2}\right] \bmod 2 \pi$.

(ii) The importance of the formulae in (3.40) and (3.41), which is the main feature of the $\mathcal{P}$-map, is that, exploiting the equilibrium for $\left(\Theta_{i}, \vartheta_{i}\right)=(0, \pi)$, the integration of ${\overline{f_{\mathcal{P}}^{n-1, n}}}^{(2)}$ and of ${\overline{\bar{f}_{\mathcal{P}}^{i, i+1}}}^{(2)}$ can be performed explicitly, switching to a suitable associated convergent Birkhoff series, as Lemma 3.4 below states. Direct integrations of ${\overline{f_{\mathcal{K}}^{n-1, n}}}^{(2)}$, for example, starting with Hamiltonian computed in 19, appear technically much more involved and, up to now, are not known. 
LEMMA 3.4. It is possible to find complex domains $\overline{\mathfrak{B}_{i}}$ with non-empty real part and a canonical, real-analytic change of coordinates

$$
\overline{\phi_{\mathfrak{i n t}}^{i}}: \quad\left(p_{i}, q_{i}, \mathrm{y}_{i}^{*}, \mathrm{x}_{i}^{*}\right) \in \overline{\mathfrak{B}_{i}} \rightarrow\left(\Theta_{i}, \vartheta_{i}, \mathrm{y}_{i}, \mathrm{x}_{i}\right)
$$

where

$$
\begin{aligned}
& \mathrm{y}_{i}^{*}:= \begin{cases}\left(\chi_{n-2}^{*}, \chi_{n-1}^{*}, \Lambda_{n-1}^{*}, \Lambda_{n}^{*}\right) & i=n-1 \\
\left(\chi_{i-1}^{*}, \chi_{i}^{*}, \chi_{i+1}^{*}, \Lambda_{i}^{*}, \Lambda_{i+1}^{*}\right) & i=1, \cdots, n-2 \& n \geq 3\end{cases} \\
& \mathrm{x}_{i}^{*}:= \begin{cases}\left(\kappa_{n-2}^{*}, \kappa_{n-1}^{*}, \ell_{n-1}^{*}, \ell_{n}^{*}\right) & i=n-1 \\
\left(\kappa_{i-1}^{*}, \kappa_{i}^{*}, \kappa_{i+1}^{*}, \ell_{i}^{*}, \ell_{i+1}^{*}\right) & i=1, \cdots, n-2 \& n \geq 3\end{cases} \\
& \mathrm{y}_{i}:= \begin{cases}\left(\chi_{n-2}, \chi_{n-1}, \Lambda_{n-1}, \Lambda_{n}\right) & i=n-1 \\
\left(\chi_{i-1}, \chi_{i}, \chi_{i+1}, \Lambda_{i}, \Lambda_{i+1}\right) & i=1, \cdots, n-2 \& n \geq 3\end{cases} \\
&(3.43) \mathrm{x}_{i}:= \begin{cases}\left(\kappa_{n-2}, \kappa_{n-1}, \ell_{n-1}, \ell_{n}\right) & i=n-1, \cdots, n-2 \& n \geq 3 \\
\left(\kappa_{i-1}, \kappa_{i}, \kappa_{i+1}, \ell_{i}, \ell_{i+1}\right) & \end{cases}
\end{aligned}
$$

such that

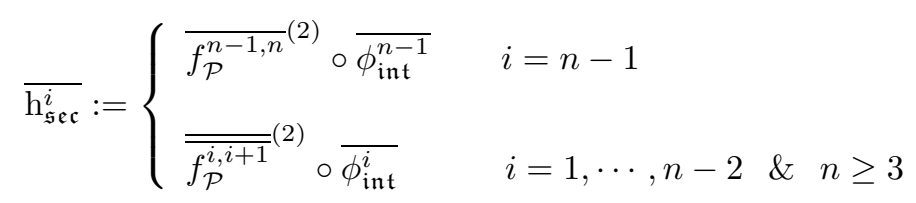

depends only on

$$
\mathrm{Y}_{i}^{*}:= \begin{cases}\left(\frac{p_{n-1}^{2}+q_{n-1}^{2}}{2}, \Lambda_{n-1}^{*}, \Lambda_{n}^{*}, \chi_{n-2}^{*}, \chi_{n-1}^{*}\right) & i=n-1 \\ \left(\frac{p_{i}^{2}+q_{i}^{2}}{2}, \Lambda_{i}^{*}, \Lambda_{i+1}^{*}, \chi_{i-1}^{*}, \chi_{i}^{*}, \chi_{i+1}^{*}\right) & i=1, \cdots, n-2 \& n \geq 3 .\end{cases}
$$

The transformation $\overline{\phi_{\mathfrak{i n t}}^{i}}$ may be chosen so as to verify

$$
\mathrm{y}_{i}^{*}=\mathrm{y}_{i}, \quad\left(\Theta_{i}, \vartheta_{i}, \mathrm{x}_{i}-\mathrm{x}_{i}^{*}\right)=\mathrm{F}_{i}\left(p_{i}, q_{i}, \mathrm{y}_{i}^{*}\right)
$$

$$
\overline{\phi_{\mathfrak{i n t}}^{i}}\left(-p_{i},-q_{i}, \mathrm{y}_{i}^{*}, \mathrm{x}_{i}^{*}\right)=\left(-\Theta_{i},-\vartheta_{i}, \mathrm{y}_{i}, \mathrm{x}_{i}\right)
$$

$$
\overline{\phi_{\mathfrak{i n t}}^{i}}\left(p_{i}, q_{i}, \mathrm{y}_{i}^{*}, \mathrm{x}_{i}^{*}\right)=\left(\Theta_{i}, \vartheta_{i}, \mathrm{y}_{i}, \mathrm{x}_{i}\right) .
$$

Lemma 3.4 is proved in the following Chapter 5.2.1 
This is a free offprint provided to the author by the publisher. Copyright restrictions may apply. 


\section{CHAPTER 4}

\section{Global Kolmogorov tori in the planetary problem}

In this chapter we show how the $\mathcal{P}$-map can be used to prove Theorem $\mathrm{A}$. We defer to the next Chapter 5 more technical parts.

\subsection{A domain of holomorphy}

A typical practice, in order to use perturbation theory techniques, is to extend Hamiltonians governing dynamical systems to the complex field, and then to study their holomorphy properties.

In this section we aim to discuss a domain of holomorphy for the perturbing function $f_{\mathcal{P}}$ in (3.37), regarded as a function of complex coordinates. We shall choose it of the following form

$$
\mathfrak{D}_{\mathcal{P}}:=\mathrm{T}_{\Theta^{+}, \vartheta+} \times\left(\mathrm{X}_{\theta} \times \overline{\mathbb{T}}_{s}^{n}\right) \times\left(\mathrm{A}_{\theta} \times \overline{\mathbb{T}}_{s}^{n}\right),
$$

where, for given positive numbers

$$
\Theta_{j}^{+}, \quad \vartheta_{j}^{+}, \quad \mathrm{G}_{i}^{ \pm}, \quad \Lambda_{i}^{ \pm}, \quad \theta_{i}, \quad s
$$

with $i=1, \cdots, n, j=1, \cdots, n-1$,

$$
\begin{aligned}
& \mathrm{T}_{\Theta+, \vartheta+}:=\left\{(\bar{\Theta}, \bar{\vartheta})=\left(\Theta_{1}, \cdots, \Theta_{n-1}, \vartheta_{1}, \cdots, \vartheta_{n-1}\right) \in \mathbb{C}^{n-1} \times \mathbb{T}_{\mathbb{C}}^{n-1}:\right. \\
&\left.\left|\vartheta_{j}-\pi\right| \leq \vartheta_{j}^{+}, \quad\left|\Theta_{j}\right| \leq \Theta_{j}^{+}, \quad \forall j=1, \cdots, n-1\right\} \\
& \mathrm{X}_{\theta}:=\left\{\chi=\left(\chi_{0}, \cdots, \chi_{n-1}\right) \in \mathbb{C}^{n}: \mathrm{G}_{j}^{-} \leq\left|\chi_{j-1}-\chi_{j}\right| \leq \mathrm{G}_{j}^{+},\right. \\
&\left.\left|\operatorname{Im}\left(\chi_{j-1}-\chi_{j}\right)\right| \leq \theta_{j} \forall j=1, \cdots, n\right\} \\
& \mathrm{A}_{\theta}:=\left\{\Lambda=\left(\Lambda_{1}, \cdots, \Lambda_{n}\right) \in \mathbb{C}^{n}: \quad \Lambda_{j}^{-} \leq\left|\Lambda_{j}\right| \leq \Lambda_{j}^{+}, \quad\left|\operatorname{Im} \Lambda_{j}\right| \leq \theta_{j}\right. \\
&\forall j=1, \cdots, n\} \\
& \overline{\mathbb{T}}_{s}:=\mathbb{T}+\mathrm{i}[-s, s]
\end{aligned}
$$

with $\chi_{n}:=0$.

The domain $\mathfrak{D}_{\mathcal{P}}$ will be determined as the intersection of the "collision-less" set, where, as functions of complex variables, the mutual distances of the planets

$$
d_{j, \mathcal{P}}:=\left\|x_{\mathcal{P}}^{(j)}-x_{\mathcal{P}}^{(j+1)}\right\|
$$

are far away from zero, with the holomorphy domain of $\mathcal{P}$, where, again as as functions of complex variables, the absolute values $\left|e_{j, \mathcal{P}}\right|$ of eccentricities in (2.18) 
are bounded away from 0 and 1 , those of the inclinations $\left|\iota_{j}\right|,\left|\mathrm{i}_{j}\right|$ in (2.14) are away from 0 and, finally, Kepler equation (2.2) provides a holomorphic solution.

The latter issue is not a peculiarity of this problem, since it naturally arises in the context of the two-body problem's equations. In the early XX century, T. Levi Civita 24] studied the holomorphy of the solution of Kepler's Equation with respect to the eccentricity. The holomorphy with respect to the mean anomaly has been investigated, using similar arguments as in [24], in [4]. Here, we address the problem of determining the holomorphy with respect to both the arguments.

Proposition 4.1. Let $\widehat{e}=0.6627 \ldots$ be the solution of

$$
0 \leq \rho \leq 1 \quad \& \quad \frac{\rho e^{\sqrt{1+\rho^{2}}}}{1+\sqrt{1+\rho^{2}}}=1 .
$$

Then for any $0<\bar{e}<\widehat{e}$, one can find a positive number $\bar{\ell}$ depending on $\bar{e}$ such that, for any $\mathrm{e}=\mathrm{e}_{1}+\mathrm{ie}_{2} \in \mathbb{C}$, with $|\mathrm{e}| \leq \bar{e}$, the complex Kepler equation

$$
\zeta-\mathrm{e} \sin \zeta=\ell
$$

has a unique solution $\zeta(\ell, \mathrm{e})$ which turns out to be real-analytic for $\ell \in \overline{\mathbb{T}}_{\bar{\ell}}$.

The following result completes the study of the holomorphy of $f_{\mathcal{P}}$.

Proposition 4.2. Let $\widehat{e}$ be as in Proposition 4.1, For any given $\underline{e}_{i}$, $\bar{e}_{i}$, with

$$
0<\underline{e}_{i}<\bar{e}_{i}<\widehat{e} \quad i=1, \cdots, n
$$

it is possible to find positive numbers

$$
\mathcal{A}_{j}, \quad \mathcal{B}_{j}, \quad \overline{\mathrm{C}}_{i}>\underline{\mathrm{C}}_{i}, \quad \bar{d}_{j}, \quad s \in(0,1), \quad \sigma \in(0,1)
$$

such that, if the following inequalities are satisfied

$\underline{\mathrm{C}}_{i} \Lambda_{i}^{+}<\mathrm{G}_{i}^{-}<\mathrm{G}_{i}^{+}<\overline{\mathrm{C}}_{i} \Lambda_{i}^{-}$

$\max \left\{\frac{\theta_{i}}{\Lambda_{i}^{-}}, \frac{\theta_{i}}{\mathrm{G}_{i}^{-}}, \sum_{i=1}^{n-1}\left|\sin ^{-1}\left(\frac{\mathrm{G}_{i}^{+}}{\mathrm{G}_{i+1}^{-}}\right)\right|, \frac{\Theta_{j}^{+}}{\mathrm{G}_{n}^{-}}, \sum_{i=1}^{n-1} \frac{\mathrm{G}_{i}^{+}}{\mathrm{G}_{n}^{-}}, \vartheta_{j}^{+},\left|\operatorname{Im} \kappa_{j}\right|,\left|\operatorname{Im} \ell_{i}\right|\right\} \leq s$

$\vartheta_{j}^{+} \leq \min \left\{\frac{\mathcal{A}}{\mathrm{G}_{n}^{+}} \sqrt{\left(\mathrm{G}_{j}^{-}\right)^{2}-\left(\underline{\mathrm{C}}_{j} \Lambda_{j}^{+}\right)^{2}}, \frac{\mathcal{B}}{\mathrm{G}_{n}^{+}} \sqrt{\left(\overline{\mathrm{C}}_{j} \Lambda_{j}^{-}\right)^{2}-\left(\mathrm{G}_{j}^{+}\right)^{2}}\right\}$,

then the eccentricities $e_{i, \mathcal{P}}$, inclinations $\iota_{i}, \mathrm{i}_{i}$ and the mutual distances $d_{i, \mathcal{P}}$ verify

$$
\underline{e}_{i} \leq\left|e_{i, \mathcal{P}}\right| \leq \bar{e}_{i}, \quad \max _{i, j}\left\{\left|\cos \iota_{i}\right|,\left|\cos i_{j}\right|\right\} \leq \sigma, \quad\left|d_{i, \mathcal{P}}\right| \geq \bar{d}
$$

Proposition 4.1 and Proposition 4.2 are proved in Appendix A.1 and A.2, respectively. We shall use them in the form below. We remark that the superexponential decay of the semi-major axes ratio will be used only in Chapter 5.2 below.

Corollary 4.1 (choice of parameters). Fix $\underline{e}_{i}<\bar{e}_{i}, c \in(0,1)$, and let $\underline{\mathcal{C}}_{i}<$ $\underline{\mathcal{C}}_{i}^{*}<\overline{\mathcal{C}}_{i}^{*}<\overline{\mathcal{C}}_{i}, \quad \mathcal{D}_{i}:=\min \left\{\mathcal{A} \sqrt{\left(\underline{\mathcal{C}}_{i}^{*}\right)^{2}-\left(\underline{\mathcal{C}}_{i}\right)^{2}}, \quad \mathcal{B} \sqrt{\left(\overline{\mathcal{C}}_{i}\right)^{2}-\left(\overline{\mathcal{C}}_{i}^{*}\right)^{2}}\right\}, \quad \mathcal{D}:=$ 
$\min _{1 \leq j \leq n-1} \frac{\mathcal{D}_{j}}{\overline{\mathcal{C}}_{n}^{*}} \frac{\mathfrak{m}_{j} \sqrt{\mathfrak{M}_{j}}}{\mathfrak{m}_{n} \sqrt{\mathfrak{M}_{n}}}, \alpha<\frac{s}{\mathcal{D}}$. Define, for $i=1, \cdots, n$ and $j=1, \cdots, n-1$,

$$
\begin{aligned}
\Lambda_{i}^{ \pm} & :=\mathfrak{m}_{i} \sqrt{\mathfrak{M}_{i} a_{i}^{ \pm}}, \quad \mathrm{G}_{i}^{+}:=\overline{\mathcal{C}}_{i}^{*} \Lambda_{i}^{-}, \quad \mathrm{G}_{i}^{-}:=\underline{\mathcal{C}}_{i}^{*} \Lambda_{i}^{+}, \quad \Theta_{j}^{+}:=s \mathrm{G}_{n}^{-}, \\
\vartheta_{j}^{+} & :=\mathcal{D}_{i} \frac{\Lambda_{i}^{-}}{\mathrm{G}_{n}^{+}} \quad \theta_{i}:=s \sqrt{\Lambda_{i}^{-}}
\end{aligned}
$$

where $a_{i}^{ \pm}$is as in $(*)$. Then $f_{\mathcal{P}}$ is real-analytic in the domain $\mathfrak{D}_{\mathcal{P}}$.

\subsection{A normal form for the planetary problem}

Definition $4.1([\mathbf{2}])$. Given $m, \nu_{1}, \cdots, \nu_{m} \in \mathbb{N}, \nu:=\nu_{1}+\cdots+\nu_{m}$, let

$$
\mathfrak{L}_{0} \supset \mathfrak{L}_{1} \supset \mathfrak{L}_{2} \supset \cdots \supset \mathfrak{L}_{m}=\{0\}
$$

be a decreasing sequence of sub-lattices of $\mathbb{Z}^{\nu}$ defined by $(4.51)$

$$
\mathfrak{L}_{0}:=\mathbb{Z}^{\nu}, \quad \mathfrak{L}_{i}:=\left\{k=\left(k_{1}, \cdots, k_{m}\right) \in \mathbb{Z}^{\nu}, \quad k_{j} \in \mathbb{Z}^{\nu_{j}}: \quad k_{1}=\cdots=k_{i}=0\right\}
$$

with $i=1, \cdots, n$. Next, given $\gamma, \gamma_{1}, \cdots, \gamma_{m}, \tau \in \mathbb{R}_{+}$, we define the set $\mathrm{D}_{\gamma_{1} \cdots \gamma_{m} ; \tau}^{\nu}$ of the $\left(\gamma_{1} \cdots \gamma_{m} ; \tau\right)$-diophantine numbers via the following formulae:

$$
\begin{aligned}
& \mathrm{D}_{\gamma ; \tau}^{\nu, K, i}:=\left\{\omega \in \mathbb{R}^{\nu}:|\omega \cdot k| \geq \frac{\gamma}{|k|^{\tau}} \quad \forall k \in \mathfrak{L}_{i-1} \backslash \mathfrak{L}_{i},|k|_{1} \leq K\right\} \\
& \mathrm{D}_{\gamma_{1} \cdots \gamma_{m} ; \tau}^{\nu, K}:=\bigcap_{i=1}^{m} \mathrm{D}_{\gamma_{i} ; \tau}^{\nu, K, i} \quad \mathrm{D}_{\gamma_{1} \cdots \gamma_{m} ; \tau}^{\nu}:=\bigcap_{K \in \mathbb{N}} \mathrm{D}_{\gamma_{1} \cdots \gamma_{m} ; \tau}^{\nu, K} .
\end{aligned}
$$

In other words $\omega=\left(\omega_{1}, \cdots, \omega_{m}\right) \in \mathrm{D}_{\gamma_{1} \cdots \gamma_{m} ; \tau}^{\nu}$ if, for any $k=\left(k_{1}, \cdots, k_{m}\right) \in \mathbb{Z}^{\nu} \backslash\{0\}$, with $k_{j} \in \mathbb{Z}^{\nu_{j}}$,

$$
|\omega \cdot k|=\left|\sum_{j=1}^{m} \omega_{j} \cdot k_{j}\right| \geq\left\{\begin{array}{l}
\frac{\gamma_{1}}{|k|^{\tau}} \quad \text { if } \quad k_{1} \neq 0 \\
\frac{\gamma_{2}}{|k|^{\tau}} \quad \text { if } \quad k_{1}=0, \quad k_{2} \neq 0 \\
\vdots \\
\frac{\gamma_{m}}{\left|k_{m}\right|^{\tau}} \quad \text { if } \quad k_{1}=\cdots=k_{m-1}=0, \cdots, k_{m} \neq 0 .
\end{array}\right.
$$

REMARK 4.1. The choice $m=1, \gamma_{1}:=\gamma$ gives the usual Diophantine set $\mathcal{D}_{\gamma, \tau}^{\nu}$. The $m=2$-case, $\mathcal{D}_{\gamma_{1}, \gamma_{2}, \tau}^{\nu}$, with $\gamma_{1}=\mathrm{O}(1)$ and $\gamma_{2}=\mathrm{O}(\mu)$, where $\mu$ is the strength of the planetary masses has been considered in $[\mathbf{2}$ for the proof of the Fundamental Theorem, mentioned in the introduction.

The following result in proven in the next Chapter 5, It is unavoidably detailed.

Proposition 4.3. Let $\mathfrak{m}_{j}, \mathfrak{M}_{j}$ be as in (3.34) and $\mathrm{m}_{j}:=\sum_{i=1}^{j-1} m_{i}$, with $j=$ $2, \cdots, n$. There exists a number $\mathfrak{c}$, depending only on $n, m_{0}, \cdots, m_{n}, a_{n}^{ \pm}, \underline{e}_{j}, \bar{e}_{j}$, and a number $0<\overline{\mathfrak{c}}<1$, depending only on $n$ such that, for any fixed positive numbers $\bar{\gamma}<1<\bar{K}, \alpha>0$ verifying

$$
\bar{K} \leq \frac{\mathfrak{c}}{\alpha^{3 / 2}}
$$


and

$$
\frac{1}{\mathfrak{c}} \max \left\{\mu\left(\frac{a_{n}^{+}}{a_{1}^{-}}\right)^{5} \frac{\bar{K}^{2 \bar{\tau}+2}}{\bar{\gamma}^{2}}, \frac{\bar{K}^{2(\bar{\tau}+1)} \alpha}{\bar{\gamma}^{2}}\right\}<1
$$

there exist natural numbers $\nu_{1}, \cdots, \nu_{2 n-1}$, with $\sum_{j} \nu_{j}=3 n-2$, open sets $B_{j}^{*} \subset$ $B_{\varepsilon_{j}}^{2}, \mathrm{X}^{*} \subset \mathrm{X}$, positive real numbers $\gamma_{1}>\cdots>\gamma_{2 n-1} \varepsilon_{1}, \cdots, \varepsilon_{n-1}, \bar{r}_{1}, \cdots, \bar{r}_{n-1}$, $\widetilde{r}_{1}, \cdots, \widetilde{r}_{n}$, a domain

$$
\mathfrak{D}_{\mathrm{n}}:=B_{\sqrt{2 \bar{r}}} \times \mathrm{X}_{\bar{r}} \times \mathcal{A}_{\widetilde{r}} \times \mathbb{T}_{\overline{\mathfrak{c} s}}^{n} \times \mathbb{T}_{\overline{\mathfrak{c}} s}^{n}
$$

a sub-domain of the form

$$
\mathfrak{D}_{\mathrm{n}}^{*}:=B_{\sqrt{2 \bar{r}}}^{*} \times \mathrm{X}_{\bar{r}}^{*} \times \mathcal{A}_{\widetilde{r}} \times \mathbb{T}_{\overline{\mathrm{c} s}}^{n} \times \mathbb{T}_{\overline{\mathrm{c} s}}^{n}
$$

verifying

$$
\text { meas } \mathfrak{D}_{\mathrm{n}}^{*} \geq\left(1-\frac{\bar{\gamma}}{\overline{\mathfrak{c}}}\right) \text { meas } \mathfrak{D}_{\mathrm{n}}
$$

a real-analytic transformation

$$
\phi_{\mathrm{n}}: \quad(p, q, \chi, \Lambda, \kappa, \ell) \in \mathfrak{D}_{\mathrm{n}}^{*} \rightarrow \mathfrak{D}_{\mathcal{P}}
$$

which conjugates $\mathrm{H}_{\mathcal{P}}$ to

$$
\mathrm{H}_{\mathrm{n}}(p, q, \chi, \Lambda, \kappa, \ell):=\mathrm{H}_{\mathcal{P}} \circ \phi_{\mathrm{n}}=\mathrm{h}_{\mathfrak{f a s t}, \mathfrak{s e c}}(p, q, \chi, \Lambda)+\mu f_{\mathfrak{e x p}}(p, q, \chi, \Lambda, \kappa, \ell)
$$

where $f_{\mathfrak{e x p}}(p, q, \chi, \Lambda, \kappa, \ell)$ is independent of $\kappa_{0}$, and the following holds.

1. The function $\mathrm{h}_{\mathfrak{f a s t}, \mathfrak{s e c}}(p, q, \chi, \Lambda)$ is a sum

$$
\mathrm{h}_{\mathfrak{f a s t}, \mathfrak{s e c}}(p, q, \chi, \Lambda)=\mathrm{h}_{\mathfrak{f a s t}}(\Lambda)+\mu \mathrm{h}_{\mathfrak{s e c}}(p, q, \chi, \Lambda)
$$

where, if

$$
\hat{\mathrm{y}}_{i}:=\left(\frac{p_{i}^{2}+q_{i}^{2}}{2}, \cdots, \frac{p_{n-1}^{2}+q_{n-1}^{2}}{2}, \chi_{i-1}, \cdots, \chi_{n-1}, \Lambda_{i}, \cdots, \Lambda_{n}\right)
$$

then $\mathrm{h}_{\mathfrak{f a s t}}$ and $\mathrm{h}_{\mathfrak{s e c}}$ are given by

$$
\mathrm{h}_{\mathfrak{f a s t}}(\Lambda)=-\sum_{j=1}^{n} \frac{\mathfrak{m}_{j}^{3} \mathfrak{M}_{j}^{2}}{2 \Lambda_{j}^{2}}-\mu \sum_{j=2}^{n} \frac{\mathfrak{M}_{j} \mathfrak{m}_{j}^{2} m_{j} \mathrm{~m}_{j}}{\Lambda_{j}^{2}}, \quad \mathrm{~h}_{\mathfrak{s e c}}(p, q, \chi, \Lambda)=\sum_{i=1}^{n-1} \mathrm{~h}_{\mathfrak{s e c}}^{i}\left(\hat{\mathrm{y}}_{i}\right)
$$

where the functions $\mathrm{h}_{\mathfrak{s e c}}^{i}$ have an analytic extension on $\mathfrak{D}_{\mathrm{n}}$ and verify

$$
\mathfrak{c} \frac{\left(a_{n-j}^{+}\right)^{2}}{\left(a_{n-j+1}^{-}\right)^{3}} \leq\left|\mathrm{h}_{\mathfrak{s e c}}^{j}\left(\hat{\mathrm{y}}_{j}\right)\right| \leq \frac{1}{\mathfrak{c}} \frac{\left(a_{n-j}^{+}\right)^{2}}{\left(a_{n-j+1}^{-}\right)^{3}} .
$$

2. The function $f_{\mathfrak{e x p}}$ satisfies

$$
\left|f_{\mathfrak{e} \mathfrak{x} \mathfrak{p}}\right| \leq \frac{1}{\mathfrak{c}} \frac{e^{-\mathfrak{c} \bar{K}}}{a_{1}^{-}}
$$


3. If $\zeta$ is $\hat{\mathrm{y}}_{1}$ deprived of $\chi_{0}$, the frequency-map

$$
\zeta \rightarrow \omega_{\mathfrak{f a s t}, \mathfrak{s e c}}(\zeta):=\partial_{\zeta} \mathrm{h}_{\mathfrak{f a s t}, \mathfrak{s e c}}(\zeta)
$$

is a diffeomorphism of $\Pi_{\zeta}\left(B_{\sqrt{2}}^{*} \times \mathrm{X}_{\bar{r}}^{*} \times \mathcal{A}_{\widetilde{r}}^{*}\right)$ and, moreover, it satisfies (4.52), with $m=2 n-1, \tau=\bar{\tau}>2$, and

$$
\begin{aligned}
& \nu_{j}:= \begin{cases}1 & j=1, \cdots, n \\
2 & j=3, n=2 \\
3 & j=n+1, n \geq 3 \\
2 & n+2 \leq j \leq 2 n-2, n \geq 4 \\
1 & j=2 n-1, n \geq 3\end{cases} \\
& \omega_{j}:= \begin{cases}\partial_{\Lambda_{j}} \mathrm{~h}_{\mathfrak{f a s t}, \mathfrak{s e c}} & j=1, \cdots, n \\
\partial_{\left(\frac{p_{1}^{2}+q_{1}^{2}}{2}, \chi_{1}\right)} \mathrm{h}_{\mathfrak{f a s t}, \mathfrak{s e c}} & j=3, n=2 \\
\partial_{\left(\frac{p_{n-1}^{2}+q_{n-1}^{2}}{2}, \chi_{n-2}, \chi_{n-1}\right)} \mathrm{h}_{\mathfrak{f a s t}, \mathfrak{s e c}} j=n+1, n \geq 3 \\
\partial_{\left(\frac{p_{2 n-j}^{2}+q_{2 n-j}^{2}}{2}, \chi_{2 n-j-1}\right)} \mathrm{h}_{\mathfrak{f a s t , \mathfrak { s e c }}} & n+2 \leq j \leq 2 n-2, n \geq 4 \\
\partial_{\frac{p_{1}^{2}+q_{1}^{2}}{2}} \mathrm{~h}_{\mathfrak{f a s t}, \mathfrak{s e c}} & j=2 n-1, n \geq 3\end{cases} \\
& \gamma_{j}:= \begin{cases}\frac{1}{a_{j}^{-}} \frac{\bar{\gamma}}{\theta_{j}} & 1 \leq j \leq n \\
\frac{\mu\left(a_{j-n}^{+}\right)^{2}}{\left(a_{j+1-n}^{-}\right)^{3}} \frac{\bar{\gamma}}{\theta_{j-n}} & n+1 \leq j \leq 2 n-1\end{cases}
\end{aligned}
$$

4. The mentioned constants are

$$
\varepsilon_{j}:=\mathfrak{c} \sqrt{\theta_{j}}, \quad \bar{r}_{j}:=\frac{\theta_{j} \bar{\gamma}}{\bar{K}^{\bar{\tau}+1}}, \quad \widetilde{r}_{i}:=\mathfrak{c} \theta_{j}
$$

with $\bar{\tau}>2$.

\subsection{A "multi-scale" KAM Theorem and proof of Theorem A}

In this section we state a "multi-scale" KAM Theorem and next we show how this theorem applies to the Hamiltonian $\mathrm{H}_{n}$ so as to obtain the proof of Theorem A.

Theorem 4.1 (Multi-scale KAM Theorem). Let $m, \ell, \nu_{1}, \cdots, \nu_{m} \in \mathbb{N}, \nu:=\nu_{1}+$ $\cdots+\nu_{m} \geq \ell, \tau_{*}>\nu, \gamma_{1} \geq \cdots \geq \gamma_{m}>0,0<4 s \leq \bar{s}<1, \rho_{1}, \cdots, \rho_{\ell}, r_{1}, \cdots, r_{\nu-\ell}$, 
$\varepsilon_{1}, \cdots, \varepsilon_{\ell}>0, B_{1}, \cdots, B_{\ell} \subset \mathbb{R}^{2}, D_{j}:=\left\{\frac{x^{2}+y^{2}}{2} \in \mathbb{R}:(x, y) \in B_{j}\right\} \subset \mathbb{R}, B:=$ $B_{1} \times \cdots \times B_{\ell} \subset \mathbb{R}^{2 \ell}, D:=D_{1} \times \cdots \times D_{\ell} \subset \mathbb{R}^{\ell}, C \subset \mathbb{R}^{\nu-\ell}, A:=D_{\rho} \times C_{r}$. Let

$$
\mathrm{H}(p, q, I, \psi)=\mathrm{h}(p, q, I)+f(p, q, I, \psi)
$$

be real-analytic on $B_{\sqrt{2 \rho}} \times C_{r} \times \mathbb{T}_{\mathfrak{s}+s}^{\nu-\ell}$, where $\mathrm{h}(p, q, I)$ depends on $(p, q)$ only via

$$
J(p, q):=\left(\frac{p_{1}^{2}+q_{1}^{2}}{2}, \cdots, \frac{p_{\ell}^{2}+q_{\ell}^{2}}{2}\right) .
$$

Assume that $\omega_{0}:=\partial_{(J(p, q), I)} \mathrm{h}$ is a diffeomorphism of $A$ with non singular Hessian matrix $U:=\partial_{(J(p, q), I)}^{2} \mathrm{~h}$ and let $U_{k}$ denote the $\left(\nu_{k}+\cdots+\nu_{m}\right) \times \nu$ submatrix of $U$, i.e., the matrix with entries $\left(U_{k}\right)_{i j}=U_{i j}$, for $\nu_{1}+\cdots+\nu_{k-1}+1 \leq i \leq \nu, 1 \leq j \leq \nu$, where $2 \leq k \leq m$. Let

$$
\begin{aligned}
& \mathrm{M} \geq \sup _{A}\|U\|, \quad \mathrm{M}_{k} \geq \sup _{A}\left\|U_{k}\right\|, \quad \overline{\mathrm{M}} \geq \sup _{A}\left\|U^{-1}\right\|, \quad E \geq\|f\|_{\rho, \bar{s}+s} \\
& \overline{\mathrm{M}}_{k} \geq \sup _{A}\left\|T_{k}\right\| \quad \text { if } \quad U^{-1}=\left(\begin{array}{l}
T_{1} \\
\vdots \\
T_{m}
\end{array}\right) \quad 1 \leq k \leq m .
\end{aligned}
$$

Define

$$
\begin{aligned}
K & :=\frac{6}{s} \log _{+}\left(\frac{E \mathrm{M}_{1}^{2} L}{\gamma_{1}^{2}}\right)^{-1} \text { where } \log _{+} a:=\max \{1, \log a\} \\
\hat{\rho}_{k} & :=\frac{\gamma_{k}}{3 \mathrm{M}_{k} K^{\tau_{*}+1}}, \hat{\rho}:=\min \left\{\hat{\rho}_{1}, \cdots, \hat{\rho}_{m}, \rho_{1}, \cdots, \rho_{\ell}, r_{1}, \cdots, r_{\nu-\ell}\right\} \\
L & :=\max \left\{\overline{\mathrm{M}}, \mathrm{M}_{1}^{-1}, \cdots, \mathrm{M}_{m}^{-1}\right\} \\
\hat{E} & :=\frac{E L}{\hat{\rho}^{2}} .
\end{aligned}
$$

Then one can find two numbers $\hat{c}_{\nu}>c_{\nu}$ depending only on $\nu$ such that, if the perturbation $f$ is so small that the following "KAM condition" holds

$$
\hat{c}_{\nu} \hat{E}<1
$$

for any $\omega \in \Omega_{*}:=\omega_{0}(D) \cap \mathcal{D}_{\gamma_{1}, \cdots, \gamma_{m}, \tau_{*}}$, one can find a unique real-analytic embedding

$$
\begin{aligned}
\phi_{\omega}: \quad \vartheta=(\hat{\vartheta}, \bar{\vartheta}) \in \mathbb{T}^{\nu} \rightarrow & \left(\hat{v}(\vartheta ; \omega), \hat{\vartheta}+\hat{u}(\vartheta ; \omega), \mathcal{R}_{\bar{\vartheta}+\bar{u}(\vartheta ; \omega)} w_{1}, \cdots, \mathcal{R}_{\bar{\vartheta}+\bar{u}(\vartheta ; \omega)} w_{\ell}\right) \\
& \in \operatorname{Re} C_{r} \times \mathbb{T}^{\nu-\ell} \times \operatorname{Re} B_{\sqrt{2 r}}^{2 \ell}
\end{aligned}
$$

where $r:=c_{\nu} \hat{E} \hat{\rho}$ such that $\mathrm{T}_{\omega}:=\phi_{\omega}\left(\mathbb{T}^{\nu}\right)$ is a real-analytic $\nu$-dimensional $\mathrm{H}$ invariant torus, on which the $\mathrm{H}$-flow is analytically conjugated to $\vartheta \rightarrow \vartheta+\omega t$. Furthermore, the map $(\vartheta ; \omega) \rightarrow \phi_{\omega}(\vartheta)$ is Lipschitz and one-to-one and the invariant set $\mathrm{K}:=\bigcup_{\omega \in \Omega_{*}} \mathrm{~T}_{\omega}$ satisfies the following measure estimate meas $\left(\operatorname{Re}\left(D_{r}\right) \times \mathbb{T}^{n} \backslash \mathrm{K}\right) \leq c_{\nu}\left(\operatorname{meas}\left(D \backslash D_{\gamma_{1}, \cdots, \gamma_{m}, \tau_{*}} \times \mathbb{T}^{n}\right)+\operatorname{meas}\left(\operatorname{Re}\left(D_{r}\right) \backslash D\right) \times \mathbb{T}^{n}\right)$, 
where $D_{\gamma_{1}, \cdots, \gamma_{m}, \tau_{*}}$ denotes the $\omega_{0}$-pre-image of $\mathcal{D}_{\gamma_{1}, \cdots, \gamma_{m}, \tau_{*}}$ in D. Finally, on $\mathbb{T}^{\nu} \times$ $\Omega_{*}$, the following uniform estimates hold

$$
\begin{aligned}
\left|v_{k}(\cdot ; \omega)-I_{k}^{0}(\omega)\right| & \leq c_{\nu}\left(\frac{\overline{\mathrm{M}}_{k}}{\overline{\mathrm{M}}}+\frac{\mathrm{M}_{k}}{\mathrm{M}_{1}}\right) \hat{E} \hat{\rho} \\
|u(\cdot ; \omega)| & \leq c_{\nu} \hat{E} s
\end{aligned}
$$

where $v_{k}$ denotes the projection of $v=(\hat{v}, \bar{v}) \in \mathbb{R}^{\nu_{1}} \times \cdots \times \mathbb{R}^{\nu_{m}}$ over $\mathbb{R}^{\nu_{k}}, \bar{v}_{k}:=\frac{\left|w_{k}\right|^{2}}{2}$ and $I^{0}(\omega)=\left(I_{1}^{0}(\omega), \cdots, I_{\nu}^{0}(\omega)\right) \in D$ is the $\omega_{0}$-pre-image of $\omega \in \Omega_{*}$.

Theorem 4.1 generalizes [6. Proposition 3] in two respects. The first generalization concerns the consideration of $m \geq 2$ scales (in [6] only the case $m=2$ was treated). The second consists of taking $\mathrm{H}$ depending also on the rectangular variables $(p, q) \in$ $B^{2 \ell}$. Such generalizations can be easily obtained, and hence will be not discussed here.

Proof of Theorem A. Let

$$
\bar{\gamma}:=\overline{\mathfrak{c}} \sqrt{\alpha}\left(\log \alpha^{-1}\right)^{\bar{\tau}+1}, \quad \bar{K}=\frac{1}{\widetilde{\mathfrak{c}}} \log \frac{1}{\alpha}
$$

where $\overline{\mathfrak{c}}$ is as in 4.55 and $\widetilde{\mathfrak{c}}$ will be fixed later. We aim to apply Theorem 4.1 to the Hamiltonian $\mathrm{H}_{\mathrm{n}}$ of Proposition 4.3, with these choices of $\bar{\gamma}$ and $\bar{K}$. To this end, we take

$$
\begin{aligned}
& \mathrm{M}_{j}= \begin{cases}\frac{1}{\mathfrak{c}_{1} a_{j}^{-} \theta_{j}^{2}} & 1 \leq j \leq n \\
\frac{\mu\left(a_{j}^{+}\right)^{2}}{\mathfrak{c}_{1}\left(a_{j+1}^{-}\right)^{3} \theta_{j}^{2}} & n+1 \leq j \leq 2 n-1\end{cases} \\
& L=\overline{\mathrm{M}}=\frac{1}{\mathfrak{c}_{2}} \frac{\theta_{1}^{2}\left(a_{2}^{+}\right)^{3}}{\mu\left(a_{1}^{-}\right)^{2}} \\
& E=\frac{1}{\mathfrak{c}_{3}} \frac{\mu}{a_{1}^{-}} e^{-\mathfrak{c} \bar{K}} \\
& K=\frac{1}{\mathfrak{c}_{4}} \log _{+}\left(\frac{1}{\bar{\gamma}^{2}} \frac{\left(a_{2}\right)^{3}}{\left(a_{1}^{-}\right)^{3}} e^{-\mathfrak{c} \bar{K}}\right)^{-1} \\
& \hat{\rho}_{j}= \begin{cases}\mathfrak{c}_{5} \frac{\bar{\gamma} \theta_{j}}{K^{\tau_{*}+1}} & 1 \leq j \leq n \\
\mathfrak{c}_{5} \frac{\bar{\gamma} \theta_{j-n}}{K^{\tau_{*}+1}} & n+1 \leq j \leq 2 n-1\end{cases} \\
& \hat{E}=\frac{1}{\mathfrak{c}_{6}} \frac{1}{\bar{\gamma}^{2}} \frac{\left(a_{2}\right)^{3}}{\left(a_{1}^{-}\right)^{3}} e^{-\mathfrak{c} \bar{K}} \hat{K}^{2\left(\tau_{*}+1\right)} \\
& \hat{\rho}:=\frac{\theta_{1} \bar{\gamma}}{\hat{K}^{\tau_{*}+1}} \quad \tau_{*}>3 n-2
\end{aligned}
$$

where $\hat{K}:=\max \{K, \bar{K}\}$. The number $\frac{1}{\bar{\gamma}^{2}} \frac{\left(a_{2}\right)^{3}}{\left(a_{1}^{-}\right)^{3}}$ can be bounded by $\frac{1}{\alpha^{N}}$ for a sufficiently large $N$ depending only on $n$. Hence, if $\tilde{\mathfrak{c}}<\frac{\mathfrak{c}}{N}$ and $\alpha<\mathfrak{c}_{6}$, we have $\hat{E}<1$ and the theorem is proved. 
This is a free offprint provided to the author by the publisher. Copyright restrictions may apply. 


\section{CHAPTER 5}

\section{Proofs}

In this chapter we provide the proof of Proposition 4.3. This is divided in two steps: normalization of fast angles and of secular coordinates.

\subsection{Normalization of fast angles}

Let $\overline{f_{\mathcal{P}}^{i j}},{\overline{f_{\mathcal{P}}^{i j}}}^{(k)}$ as in Lemma 3.1, and let

$$
{\overline{f_{\mathcal{P}}^{i j}}}^{(\geq 2)}:=\overline{f_{\mathcal{P}}^{i j}}-{\overline{f_{\mathcal{P}}^{i j}}}^{(0)} .
$$

Proposition 5.1. There exist two small numbers $\widehat{\mathfrak{c}}, \mathfrak{c}_{1}$, where $\widehat{\mathfrak{c}}$ depends only on $n$, while $\mathfrak{c}_{1}$ depends only on $n, m_{1}, \cdots, m_{n}$, such that, if the inequality in (4.53) and

$$
\frac{1}{\mathfrak{c}} \mu \bar{K}\left(\frac{a_{n}^{+}}{a_{1}^{-}}\right)^{\frac{3}{2}}<1
$$

hold, one can find a real-analytic and symplectic transformation

$$
\phi_{\mathfrak{f a s t}}: \quad(\bar{\Theta}, \bar{\vartheta}, \chi, \Lambda, \kappa, \ell) \in \mathfrak{D}_{\mathfrak{f a s t}}:=\mathrm{T}_{\widehat{\mathfrak{c}} \Theta^{+}, \widehat{\mathfrak{c}} \vartheta+} \times \mathrm{X}_{\widehat{\mathfrak{c}} \theta} \times \mathcal{A}_{\widehat{\mathfrak{c}} \theta} \times \mathbb{T}_{\widehat{\mathfrak{c}} s}^{n} \times \mathbb{T}_{\widehat{\mathfrak{c}} s}^{n} \rightarrow \mathfrak{D}_{\mathcal{P}}
$$

which conjugates $\mathrm{H}_{\mathcal{P}}$ to

$$
\begin{aligned}
& \mathrm{H}_{\mathfrak{f a s t}, \mathfrak{e} \mathfrak{x}}(\bar{\Theta}, \chi, \Lambda, \bar{\vartheta}, \kappa, \ell) \\
& \quad:=\mathrm{H}_{\mathcal{P}} \circ \phi_{\mathfrak{f a s t}}=\mathrm{h}_{\mathfrak{f a s t}}(\Lambda)+\mu f_{\mathfrak{f a s t}}(\bar{\Theta}, \chi, \Lambda, \bar{\vartheta}, \kappa)+\mu f_{\mathfrak{f a s t}, \mathfrak{e} \mathfrak{p}}(\bar{\Theta}, \chi, \Lambda, \bar{\vartheta}, \kappa, \ell)
\end{aligned}
$$

where $\mathrm{h}_{\mathfrak{f a s t}}$ is as in Proposition 4.3, and

$$
f_{\mathfrak{f a s t}}:=\sum_{i=1}^{n-1} f_{\mathfrak{f a s t}}^{i}, \quad f_{\mathfrak{f a s t}, \mathfrak{e x p}}:=\sum_{i=1}^{n-1} f_{\mathfrak{f a s t}, \mathfrak{e x p}}^{i} .
$$

Here,

1. The "fast frequency-map"

$$
\omega_{\mathfrak{f a s t}}:=\partial \mathrm{h}_{\mathfrak{f a s t}}
$$

is a diffeomorphism of $\mathcal{A}$ with non-vanishing Jacobian matrix on $\mathcal{A}_{\hat{\mathfrak{c}} \theta}$ and, moreover,

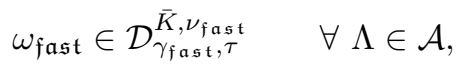

with

$$
\gamma_{\mathfrak{f a s t}}:=\left(\gamma_{1}, \cdots, \gamma_{n}\right) \quad \nu_{\mathfrak{f a s t}}:=\left(\nu_{1}, \cdots, \nu_{n}\right)
$$

and $\nu_{i}, \gamma_{i}$ as in (4.56); 
2. the functions $f_{\mathfrak{f a s t}}^{i}, f_{\mathfrak{f a s t}, \mathfrak{e r p}}^{i}$ do not depend on $\kappa_{0}$; the $f_{\mathfrak{f a} \mathfrak{s t}}^{i}$ 's are given by

$$
f_{\mathfrak{f a s t}}^{i}=f_{\mathfrak{f a s t}}^{i}\left(\mathrm{t}_{i}, \mathrm{y}_{i}, \mathrm{x}_{i}\right)={\overline{f_{\mathcal{P}}^{i}}}^{(\geq 2)}\left(\mathrm{t}_{i}, \mathrm{y}_{i}, \mathrm{x}_{i}\right)+\widetilde{f_{\mathfrak{f a s t}}^{i}}\left(\mathrm{t}_{i}, \mathrm{y}_{i}, \mathrm{x}_{i}\right), \quad i=1, \cdots, n-1,
$$

with

$$
\begin{aligned}
{\overline{f_{\mathcal{P}}^{i}}}^{(\geq 2)} & :=\sum_{j=i+1}^{n}{\overline{f_{\mathcal{P}}^{i j}}}^{(\geq 2)} \\
\mathrm{t}_{i} & :=\left(\Theta_{i}, \cdots, \Theta_{n-1}, \vartheta_{i}, \cdots, \vartheta_{n-1}\right), \\
\mathrm{y}_{i} & :=\left(\chi_{i-1}, \cdots, \chi_{n-1}, \Lambda_{i}, \cdots, \Lambda_{n}\right) \\
\mathrm{x}_{i} & :=\left(\kappa_{i}, \cdots, \kappa_{n-1}\right) .
\end{aligned}
$$

In particular, $\widetilde{f_{\mathfrak{f a s t}}^{i}}$ do not depend on $\ell_{1}, \cdots, \ell_{n}$;

3. finally, $\widetilde{f_{\mathfrak{f a s t}}^{i}}, f_{\exp , \mathfrak{f a s t}}^{i}$ satisfy the following bounds

$$
\left\|\widetilde{f_{\mathfrak{f a s t}}^{i}}\right\|_{\mathfrak{D}_{\mathfrak{f} \mathfrak{s} \mathfrak{s}}} \leq \frac{1}{\mathfrak{c}_{1}} \mu \bar{K}\left(\frac{a_{n}^{+}}{a_{1}^{-}}\right)^{\frac{3}{2}} \frac{1}{a_{i+1}^{-}}, \quad\left\|f_{\mathfrak{f a} \mathfrak{a t}, \mathfrak{e x p}}^{i}\right\|_{\mathfrak{D}_{\mathfrak{f} a \mathfrak{s t}}} \leq \frac{1}{\mathfrak{c}_{1}} \frac{e^{-\widehat{\mathfrak{c}} \bar{K} s}}{a_{i+1}^{-}} .
$$

Let $\mathrm{L}_{0}, \cdots, \mathrm{L}_{n}$ be defined as $\mathfrak{L}_{i}$ in (4.51), with $\nu=m=n$ and $\nu_{1}=\cdots=\nu_{n}=1$.

LEMMA 5.1. If $\bar{K}$ verifies the inequality in (4.53), then one can find a number $\mathfrak{c}_{3}$, depending only on $m_{0}, \cdots, m_{n}$, such that $\left|\omega_{\mathrm{k}, \mathfrak{f a s t}}(\Lambda) \cdot k\right| \geq \frac{\mathfrak{c}_{3}}{\left(a_{j}^{+}\right)^{3 / 2}} \quad \forall k \in \mathrm{L}_{j-1} \backslash \mathrm{L}_{j}, \quad|k| \leq \bar{K}, \quad \forall \Lambda \in \mathrm{A}_{\theta}, \quad \forall j=1, \cdots, n$.

ProOF. For $\Lambda \in \mathrm{A}_{\theta}, \omega_{\mathrm{k}, \mathfrak{f a} \mathfrak{s t}, \mathrm{j}}:=\frac{\mathfrak{M}_{j}^{2} \mathfrak{m}_{j}^{3}}{\Lambda_{j}^{3}}$ verifies $\frac{\sqrt{\mathfrak{M}}_{j}}{\left(a_{j}^{+}\right)^{3 / 2}} \leq\left|\omega_{\mathrm{k}, \mathfrak{f a} \mathfrak{s t}, \mathrm{j}}\right| \leq \frac{{\sqrt{\mathfrak{M}_{j}}}_{\left(a_{j}^{-}\right)^{3 / 2}}}{}$

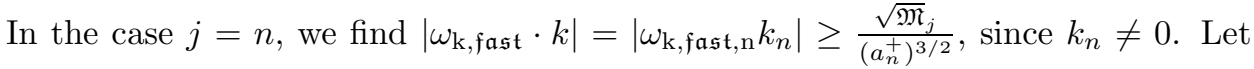
then $j \neq n$. For $k \in \mathrm{L}_{j-1} \backslash \mathrm{L}_{j}, k_{j} \neq 0$, so, inequality (4.53), with $\mathfrak{c}_{2} \leq \frac{\min _{j} \sqrt{\mathfrak{M}_{j}}}{\max _{j} \sqrt{\mathfrak{M}_{j}}}$, and (4.50) imply

$$
\bar{K} \leq \frac{\min _{j} \sqrt{\mathfrak{M}_{j}}}{\max _{j} \sqrt{\mathfrak{M}_{j}}} \min _{1 \leq j \leq n-1}\left(\frac{a_{j+1}^{-}}{a_{j}^{+}}\right)^{3 / 2}
$$

and hence

$$
\begin{aligned}
\left|\omega_{\mathrm{k}, \mathfrak{f a s t}} \cdot k\right| & =\left|\sum_{i=j}^{n} \omega_{\mathrm{k}, \mathfrak{f a s t}, \mathrm{i}} k_{i}\right| \geq \inf _{\mathcal{A}_{\theta}}\left|\omega_{\mathrm{k}, \mathfrak{f a} \mathfrak{s t}, \mathrm{j}}\right|-\bar{K} \max _{j<i \leq n} \sup _{\mathcal{A}_{\theta}}\left|\omega_{\mathrm{k}, \mathfrak{f a} \mathfrak{s t}, \mathrm{i}}\right| \\
& \geq \frac{{\sqrt{\mathfrak{M}_{j}}}_{j}}{\left(a_{j}^{+}\right)^{3 / 2}}-\bar{K} \frac{\max _{i>j} \sqrt{\mathfrak{M}_{i}}}{\left(a_{j+1}^{-}\right)^{3 / 2}} \geq \frac{\sqrt{\mathfrak{M}_{j}}}{2\left(a_{j}^{+}\right)^{3 / 2}} e q
\end{aligned}
$$

Proof of Proposition [5.1. The proof proceeds by recursion, in $n$ steps. We describe the $h^{\text {th }}$ step of this recursion, with $h=1, \cdots, n$. We start with a Hamiltonian of the form

$$
\mathrm{H}_{h-1}=\mathrm{h}_{\mathfrak{f a s t}}^{0}+\mu f_{h-1}
$$


where $\mathrm{h}_{\mathfrak{f a s t}}^{0}$ is as in (3.38), and a domain

$$
\mathfrak{D}_{h-1}=\mathrm{T}_{\Theta^{+(h-1)}, \vartheta+(h-1)} \times \mathrm{X}_{\theta^{(h-1)}} \times \mathcal{A}_{\theta^{(h-1)}} \times \mathbb{T}_{s^{(h-1)}}^{n} \times \mathbb{T}_{s^{(h-1)}}^{n} .
$$

When $h=1$, we take $\mathrm{H}_{0}:=\mathrm{H}_{\mathcal{P}}, \Theta_{+}^{(0)}:=\Theta^{+}, \vartheta_{+}^{(0)}:=\vartheta^{+}, \theta^{(0)}:=\theta, s^{(0)}:=s$, $f_{0}:=f_{\mathcal{P}}$ and we decompose

$$
f_{0}:=\widehat{f}_{0}:=\sum_{i=1}^{n-1} \widehat{f_{0}^{i}} \quad \text { with } \quad f_{0}^{i}:=\sum_{j=i+1}^{n} f_{\mathcal{P}}^{i j} .
$$

We observe that $\widehat{f}_{0}^{i}$ depends on the coordinates

$$
\begin{aligned}
& \Theta_{i}, \quad \cdots, \quad \Theta_{n-1}, \quad \chi_{i-1}, \quad \cdots, \chi_{n-1}, \quad \Lambda_{i}, \quad \cdots, \quad \Lambda_{n} \\
& \vartheta_{i}, \quad \cdots, \quad \vartheta_{n-1}, \quad \kappa_{i}, \quad \cdots, \quad \kappa_{n-1}, \quad \ell_{i}, \cdots, \quad \ell_{n} .
\end{aligned}
$$

For $n \geq 3$ and $2 \leq h \leq n-1$, we assume, inductively, that $f_{h-1}$ is a sum

$$
f_{h-1}=\widehat{f_{h-1}}+f_{\mathfrak{e x p}, h-1}=\sum_{1 \leq i \leq n} \widehat{f_{h-1}^{i}}+\sum_{1 \leq i \leq n} f_{\mathfrak{e} \mathfrak{x} \mathfrak{p}, h-1}^{i},
$$

where, in turn,

$$
\widehat{f_{h-1}^{i}}=\widetilde{f_{h-1}^{i}}+\widetilde{f_{h-1}^{i}}
$$

with $\overline{f_{h-1}^{i}}, \widetilde{f_{h-1}^{i}}$ depending only on the coordinates

$$
\begin{aligned}
& \Theta_{i}, \quad \cdots, \quad \Theta_{n-1}, \quad \chi_{i-1}, \cdots, \chi_{n-1}, \quad \Lambda_{i}, \quad \cdots, \quad \Lambda_{n} \\
& \vartheta_{i}, \quad \cdots, \quad \vartheta_{n-1}, \quad \kappa_{i}, \quad \cdots, \quad \kappa_{n-1}, \quad \ell_{i \vee h}, \quad \cdots, \quad \ell_{n}
\end{aligned}
$$

and $\overline{f_{h-1}^{i}}, \widetilde{f_{h-1}^{i}}, f_{\mathfrak{e x p}, h-1}$ verifying the following bounds and identities

$$
\begin{aligned}
\overline{f_{h-1}^{i}} & =\Pi_{\mathrm{L}_{h-1}} T_{\bar{K}} \widehat{f_{h-2}^{i}} \\
\left\|\widetilde{f_{h-1}^{i}}\right\|_{\mathfrak{D}_{h-1}} & \leq \mathfrak{C}_{1, h-1} \mu \bar{K}\left(\frac{a_{n}^{+}}{a_{1}^{-}}\right)^{\frac{3}{2}}\left\|\widehat{f_{h-2}^{i}}\right\|_{\mathfrak{D}_{h-2}} \\
\left\|f_{\mathfrak{e x} \mathfrak{i}, h-1}^{i}\right\|_{\mathfrak{D}_{h-1}} & \leq \mathfrak{C}_{2, h-1} e^{-K s^{(h)}}\left\|\widehat{f_{h-2}^{i}}\right\|_{\mathfrak{D}_{h-2}} .
\end{aligned}
$$

Here $\Pi_{L_{h}}$ denotes the projection over the module $\mathrm{L}_{h}$. In any case, $h=1$, or $2 \leq h \leq n-1$, we focus on the Hamiltonian

$$
\widehat{\mathrm{H}_{h-1}}=\mathrm{h}_{\mathfrak{f a s t}}^{0}+\mu \widehat{f_{h-1}}=\mathrm{h}_{\mathfrak{f a s t}}^{0}+\mu \sum_{i=1}^{n-1} \widehat{f_{h-1}^{i}} .
$$

Our purpose is to apply Proposition D.1 to this Hamiltonian, in the case that the abstract system (D.133) does not depend on the coordinates $(p, q)$. To this end, we take the coordinates

$$
I:=\Lambda, \quad \varphi:=\ell, \quad \eta:=(\bar{\Theta}, \chi), \quad \xi:=(\bar{\vartheta}, \kappa),
$$


the functions $f_{i}$ in (D.135) to be the $\widehat{f_{h-1}^{n-i}}$, and

$$
\begin{aligned}
& N=n-1, \quad \nu=n, \quad m_{i}:=2 i \\
& \left(I_{1}, \cdots, I_{\nu}\right):=\left(\Lambda_{n}, \cdots, \Lambda_{1}\right) \\
& \left(\varphi_{1}, \cdots, \varphi_{\nu_{i}}\right):=\left(\ell_{n}, \cdots, \ell_{\max \{n-i, h\}}\right) \\
& \left(\eta_{1}, \cdots, \eta_{m_{i}}\right):=\left(\Theta_{n-1}, \cdots, \Theta_{n-i}, \chi_{n-1}, \cdots, \chi_{n-i-1}\right) \\
& \left(\xi_{1}, \cdots, \xi_{m_{i}}\right):=\left(\vartheta_{n-1}, \cdots, \vartheta_{n-i}, \kappa_{n-1}, \cdots, \kappa_{n-i}\right) \\
& u_{i}:=\left(\Lambda_{n}, \cdots, \Lambda_{1}, \Theta_{n-1}, \cdots, \Theta_{n-i}, \chi_{n-1}, \cdots, \chi_{n-i-1}, \vartheta_{n-1}, \cdots, \vartheta_{n-i}\right. \\
& \left.\quad \kappa_{n-1}, \cdots, \kappa_{n-i}\right) .
\end{aligned}
$$

The non-resonance assumption (D.134) for $\omega=\omega_{\mathrm{k}, \text { fast }}=\partial_{\Lambda} \mathrm{h}_{\mathrm{k}, \text { fast }}$, with

$$
\mathfrak{Z}_{i}=\mathrm{L}_{h-1}, \quad \mathfrak{Z}=\cup_{i} \mathfrak{Z}_{i}=\mathrm{L}_{h-1}, \quad \mathfrak{L}=\mathrm{L}_{h} \quad K=\bar{K}
$$

is ensured by Lemma 5.1 , with

$$
\mathfrak{a}=\frac{\mathfrak{c}_{3}}{\left(a_{h}^{+}\right)^{3 / 2}}, \quad A=\mathcal{A}, \quad r=\theta_{1}^{(h-1)} .
$$

Now we have to check condition (D.139). In the case $2 \leq h \leq n-1$ the inductive assumptions (5.65) and assumption (5.58) imply

$$
\begin{aligned}
\left\|\widehat{f_{h-1}^{i}}\right\|_{\mathfrak{D}_{h-1}} & \leq\left\|\widehat{f_{h-1}^{i}}\right\|_{\mathfrak{D}_{h-1}}+\left\|\widetilde{f_{h-1}^{i}}\right\|_{\mathfrak{D}_{h-1}} \leq\left(1+\mathfrak{C}_{1} \mu \bar{K}\left(\frac{a_{n}^{+}}{a_{1}^{-}}\right)^{\frac{3}{2}}\right)\left\|\widehat{f_{h-2}^{i}}\right\|_{\mathfrak{D}_{h-2}} \\
& \leq \cdots \leq\left(1+\mathfrak{C}_{1, h-1} \mathfrak{c}_{1}\right)^{h-1}\left\|\widehat{f}_{0}^{i}\right\|_{\mathfrak{D}_{0}} \leq \frac{\mathfrak{C}_{4, h-1}}{a_{i}^{-}}=: E_{i} .
\end{aligned}
$$

An analogous bound holds also for $h=1$. The numbers $c_{i}$ and $d_{i}$ in (D.138) may be evaluated as

$$
c_{i}=e(1+2 i e) / 2 \quad d_{i}=\min \left\{\theta_{1}^{(h-1)} s^{(h-1)}, \Theta_{i}^{+(h-1)} \vartheta_{i}^{+(h-1)}\right\}=\mathfrak{c}_{2} \theta_{1}^{(h-1)} .
$$

From these bounds it is immediate to see that inequality (D.139) is implied by (5.58), provided $\mathfrak{c}_{1}<2^{-7} \frac{6}{7}\left(\frac{8}{9}\right)^{n-2} \mathfrak{c}_{2} /\left(\mathfrak{C}_{4} c_{n}\right)$. Then Proposition D.1 applies. Its thesis implies that $\widehat{\mathrm{H}_{h-1}}$ in (5.66) can be conjugated to a suitable $\mathrm{H}_{h}^{*}=\mathrm{h}_{\mathrm{k}, \mathfrak{f a s t}}+\mu f_{h}^{*}$, where $f_{h}^{*}$ verifies equalities and inequalities in (5.64)-(5.65) with $h$ replaced by $h+1$ and $\mathfrak{C}_{1, h-1}, \mathfrak{C}_{2, h-1}$ replaced by suitable $\mathfrak{C}_{1, h}^{*}, \mathfrak{C}_{2, h}^{*}$. Then, applying the same transformation to $\mathrm{H}_{h-1}$ in (5.63), we shall conjugate $\mathrm{H}_{h-1}$ to $\mathrm{H}_{h}=\mathrm{h}_{\mathrm{k}, \mathfrak{f a s t}}+\mu f_{h}$, where $f_{h}$ satisfies the same equalities and inequalities as $f_{h}^{*}$, with suitable $\mathfrak{C}_{1, h} \geq$ $\mathfrak{C}_{1, h}^{*}, \mathfrak{C}_{2, h} \geq \mathfrak{C}_{2, h}^{*}$.

After we have performed $n$ steps, we let $\mathfrak{D}_{\mathfrak{f a s t}}:=\mathfrak{D}_{n}, \mathrm{H}_{\mathfrak{f a s t}, \mathfrak{e x p}}:=\mathrm{H}_{n}, f_{\mathfrak{f a s t}}^{i}:=\widehat{f_{n}^{i}}$, $\widetilde{f_{\mathfrak{f a s t}}^{i}}:=f_{\mathfrak{f a s t}}^{i}-\overline{f_{\mathcal{P}}^{i}}, f_{\mathfrak{f a} \mathfrak{s t}, \mathfrak{e} \mathfrak{p} \mathfrak{p}}^{i}:=f_{\exp , \mathrm{n}}^{i}, \widehat{f}_{\mathfrak{f a s t}}:=\sum_{i=1}^{n-1} \widehat{f}^{i}, \widetilde{f}_{\mathfrak{f a s t}}:=\sum_{i=1}^{n-1} \widetilde{f}^{i}, f_{\mathfrak{f a} \mathfrak{s t}, \mathfrak{e x p}}:=$ $\sum_{i=1}^{n-1} f_{\mathfrak{f a s t}, \mathfrak{e x p}}^{i}$, with $\overline{f_{\mathcal{P}}^{i}}:=\sum_{j=i+1}^{n} \overline{f_{\mathcal{P}}^{i j}}$. Therefore,

$$
\mathrm{H}_{\mathfrak{f a s t}}=\mathrm{h}_{\mathfrak{f a} \mathfrak{a t}}^{(0)}+\mu\left(\widehat{f}_{\mathfrak{f a s t}}+f_{\mathfrak{e x p}, \mathfrak{f a} \mathfrak{s t}}\right)=\mathrm{h}_{\mathfrak{f a s t}}^{(0)}+\mu\left(\sum_{1 \leq i<j \leq n} \overline{f_{\mathcal{P}}^{i j}}+\widetilde{f}_{\mathfrak{f a s t}}+f_{\exp , \mathfrak{f a s t})}\right)
$$

reduces to (5.59) and the formulae given below, using (5.57).

It remains to check the bound on the left in (5.62) (the one on the right follows by construction). This follows by telescopic arguments. Indeed, 


$$
\begin{aligned}
\left\|\widetilde{f_{\mathfrak{f a s t}}^{i}}\right\|_{\mathfrak{D}_{n}} & =\left\|f_{\mathfrak{f a s t}^{i}}^{i}-\overline{f_{\mathcal{P}}^{i}}\right\|_{\mathfrak{D}_{n}}=\left\|\widehat{f_{n}^{i}}-\overline{f_{\mathcal{P}}^{i}}\right\|_{\mathfrak{D}_{n}}=\left\|\Pi_{\mathrm{L}_{n}} \widehat{f_{n}^{i}}-\Pi_{\mathrm{L}_{n}} f_{\mathcal{P}}^{i}\right\|_{\mathfrak{D}_{n}} \\
& \leq \sum_{h=1}^{n}\left\|\Pi_{\mathrm{L}_{n}} \widehat{f_{h}^{i}}-\Pi_{\mathrm{L}_{n}} T_{\bar{K}} \widehat{f_{h-1}^{i}}\right\|_{\mathfrak{D}_{n}} \\
& =\sum_{h=1}^{n}\left\|\Pi_{\mathrm{L}_{n}} \widehat{f_{h}^{i}}-\Pi_{\mathrm{L}_{n}} \Pi_{\mathrm{L}_{h}} T_{\bar{K}} \widehat{f_{h-1}^{i}}\right\|_{\mathfrak{D}_{n}} \\
& \leq \sum_{h=1}^{n}\left\|\widehat{f_{h}^{i}}-\Pi_{\mathrm{L}_{h}} T_{\bar{K}} \widehat{f_{h-1}^{i}}\right\|_{\mathfrak{D}_{n}} \\
& \leq \sum_{h=1}^{n}\left\|\widehat{f_{h}^{i}}-\Pi_{\mathrm{L}_{h}} T_{\bar{K}} \widehat{f_{h-1}^{i}}\right\|_{\mathfrak{D}_{h}} \\
& \leq \mu \bar{K}\left(\frac{a_{n}^{+}}{a_{1}^{-}}\right)^{\frac{3}{2}} \frac{\sum_{h=1}^{n}{\widehat{\mathfrak{C}_{1, h}}}_{a_{4, h-1}^{-}}}{a_{i+1}^{-}} .
\end{aligned}
$$

Here, we have used the second bound in (5.65), (5.67), that $\widehat{f_{n}^{i}}$ does not depend on $\ell_{1}, \cdots, \ell_{n}$, and, finally, $\Pi_{\mathrm{L}_{n}}=\Pi_{\mathrm{L}_{n}} T_{\bar{K}}=\Pi_{\mathrm{L}_{n}} \Pi_{\mathrm{L}_{h}}$, for all $1 \leq h \leq n$.

\subsection{Secular normalizations}

Consider the following truncation

$$
\mathrm{H}_{\mathfrak{f a s t}}(\bar{\Theta}, \chi, \Lambda, \bar{\vartheta}, \kappa):=\mathrm{h}_{\mathfrak{f a s t}}(\Lambda)+\mu f_{\mathfrak{f a s t}}(\bar{\Theta}, \chi, \Lambda, \bar{\vartheta}, \kappa)
$$

of the Hamiltonian $\mathrm{H}_{\mathfrak{f a s t}, \mathfrak{e x p}}$ in (5.59). The purpose of this section is to describe an iterative scheme which, after $(n-1)$ steps, conjugates $\mathrm{H}_{\mathfrak{f a s t}}$ to a close-to be integrable system, with an arbitrarily small remainder.

Let us firstly establish the following notation.

- Given a Taylor-Fourier expansion of the form

$$
g(p, q, \kappa)=\sum_{\substack{(a, b) \in \mathbb{N}^{2} m_{1} \\ k \in \mathbb{Z}^{m_{2}}}} g_{a, b, k}\left(\frac{p-\mathrm{i} q}{\sqrt{2}}\right)^{a}\left(\frac{p+\mathrm{i} q}{\sqrt{2} \mathrm{i}}\right)^{b} e^{\mathrm{i} k \cdot \kappa} \quad(p, q, \kappa) \in B^{2 m_{1}}(0) \times \mathbb{T}^{m_{2}}
$$

we denote as

$$
\Pi_{p, q, \kappa} g:=\sum_{a \in \mathbb{N}^{m_{1}}} g_{0, a, a}\left(\frac{p^{2}+q^{2}}{2 \mathrm{i}}\right)^{a} .
$$

Proposition 5.2. There exists number $\overline{\mathfrak{c}}_{h}$, depending only on $n, m_{0}, \cdots, m_{n}$, $a_{n}^{ \pm}$such that, for any $h=1, \cdots, n-1$ and any $\bar{K}, \bar{\gamma}>0$ such that (4.54) hold with $\mathfrak{c}$ replaced by $\overline{\mathfrak{c}}_{h}$, one finds open sets

$$
B_{j}^{*} \subset B_{\varepsilon_{j}}^{2}, \quad \mathrm{G}_{j}^{*} \subset \mathrm{G}_{j}:=\left[\mathrm{G}_{j}^{+}, \mathrm{G}_{j}^{+}\right], \quad j=n-h, \cdots, n-1
$$

verifying

$$
\operatorname{meas}\left(B_{j}^{*} \times \mathrm{G}_{j}^{*}\right) \geq\left(1-\frac{\bar{\gamma}}{\overline{\mathfrak{c}}_{h}}\right) \text { meas }\left(B_{\varepsilon_{j}}^{2} \times \mathrm{G}_{j}\right)
$$

such that, defining 


$$
\mathrm{T}_{\overline{\mathfrak{c}}_{h} \theta}^{h}:=\left\{\begin{array}{ccc}
\left\{\left(\Theta_{1}, \cdots, \Theta_{n-h-1}, \vartheta_{1}, \cdots, \vartheta_{n-h-1}\right)\right. & \\
& \in \mathbb{C}^{n-1} \times \mathbb{T}_{\mathbb{C}}^{n-1}: & \\
& \left|\vartheta_{j}-\pi\right| \leq \overline{\mathfrak{c}}_{h} \frac{\theta_{j}}{\mathrm{G}_{n}^{-}}, \quad\left|\Theta_{j}\right| \leq \overline{\mathfrak{c}}_{h} \mathrm{G}_{n}^{+} & \\
& \forall j=1, \cdots, n-h-1\} & n \geq 3,1 \leq h<n-2 \\
\emptyset & & \text { otherwise }
\end{array}\right.
$$

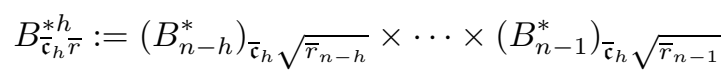$$
\mathrm{X}_{\overline{\mathfrak{c}}_{h} \theta, \overline{\mathfrak{c}}_{h} \bar{r}}^{* h}:=\left\{\chi=\left(\chi_{0}, \cdots, \chi_{n-1}\right): \quad \chi_{i-1}-\chi_{i} \in\left(\mathrm{G}_{i}^{*}\right)_{\overline{\mathfrak{c}}_{h} \theta_{i}}, \chi_{j-1}-\chi_{j} \in\left(\mathrm{G}_{j}\right)_{\overline{\mathfrak{c}}_{h} \bar{r}_{j}}\right.
$$$$
\left.\forall i=1, \cdots, n-h-1, j=n-h, \cdots, n, \quad \chi_{n}:=0\right\}
$$$$
\mathfrak{D}_{\mathfrak{s e c}}^{h}:=\mathrm{T}_{\overline{\mathfrak{c}} \theta}^{h} \times B_{\overline{\mathfrak{c}} \varepsilon}^{* h} \times \mathrm{X}_{\overline{\mathfrak{c}} \theta, \overline{\mathfrak{c}} r}^{* h} \times \mathrm{A}_{\overline{\mathfrak{c}}_{h} \widetilde{r}} \times \mathbb{T}_{\overline{\mathfrak{c}}_{h} s}^{n} \times \mathbb{T}_{\overline{\mathfrak{c}}_{h} s}^{n}
$$

a real-analytic transformation

$$
\Phi_{\mathfrak{s e c}, h}: \quad \mathfrak{D}_{\mathfrak{s e c}}^{h} \rightarrow \mathfrak{D}_{\mathfrak{f a s t}},
$$

may be found, which conjugates $f_{\mathfrak{f a s t}}$ to a new function

$$
f_{\mathfrak{s e c}, h}:=f_{\mathfrak{f a s t}} \circ \Phi_{\mathfrak{s e c}, h}
$$

enjoying the following properties.

1. Denoting by $\left(\mathrm{t}^{(h)}, \mathrm{z}^{(h)}, \mathrm{y}^{(h)}, \mathrm{x}^{(h)}\right)$, where

$$
\begin{aligned}
& \mathrm{t}^{(h)}=\left(\Theta^{(h)}, \vartheta^{(h)}\right)=\left(\Theta_{1}^{(h)}, \cdots, \Theta_{n-h-1}^{(h)}, \vartheta_{1}^{(h)}, \cdots, \vartheta_{n-h-1}^{(h)}\right) \\
& \mathrm{z}^{(h)}=\left(p^{(h)}, q^{(h)}\right)=\left(p_{n-h}^{(h)}, \cdots, p_{n-1}^{(h)}, q_{n-h}^{(h)}, \cdots, q_{n-1}^{(h)}\right) \\
& \mathrm{y}^{(h)}=\left(\chi^{(h)}, \Lambda^{(h)}\right)=\left(\chi_{0}^{(h)}, \cdots, \chi_{n-1}^{(h)}, \Lambda_{1}^{(h)}, \cdots, \Lambda_{n}^{(h)}\right) \\
& \mathrm{x}^{(h)}=\left(\kappa^{(h)}, \ell^{(h)}\right)=\left(\kappa_{0}^{(h)}, \cdots, \kappa_{n-1}^{(h)}, \ell_{1}^{(h)}, \cdots, \ell_{n}^{(h)}\right),
\end{aligned}
$$

coordinates on $\mathfrak{D}_{\mathfrak{s e c}}^{h}$ then $\Phi_{\mathfrak{s e c}, h}$ is co-variant with the symmetry:

$$
\begin{aligned}
& \Phi_{\mathfrak{s e c}, h}\left(-\mathrm{t}^{(h)},-\mathrm{z}^{(h)}, \mathrm{y}^{(h)}, \mathrm{x}^{(h)}\right)=\left(-\mathrm{t}^{(0)}, \mathrm{y}^{(0)}, \mathrm{x}^{(0)}\right) \quad \text { if } \\
& \Phi_{\mathfrak{s e c}, h}\left(\mathrm{t}^{(h)}, \mathrm{z}^{(h)}, \mathrm{y}^{(h)}, \mathrm{x}^{(h)}\right)=\left(\mathrm{t}^{(0)}, \mathrm{y}^{(0)}, \mathrm{x}^{(0)}\right)
\end{aligned}
$$

and hence, $f_{\mathfrak{s e c}, h}$ is even around

$$
\mathrm{t}^{(h)}=(0, k \pi), \quad \mathrm{z}^{(h)}=0 \quad k \in\{0,1\}^{n-h-1}
$$




\section{Defining}

$$
\begin{aligned}
& \mathrm{t}_{i}^{(h)}:=\left\{\begin{array}{l}
\left(\Theta_{i}^{(h)}, \cdots, \Theta_{n-h-1}^{(h)}, \vartheta_{i}^{(h)}, \cdots, \vartheta_{n-h-1}^{(h)}\right) \quad i \leq n-h-1 \\
\emptyset \quad \text { otherwise }
\end{array}\right.
\end{aligned}
$$

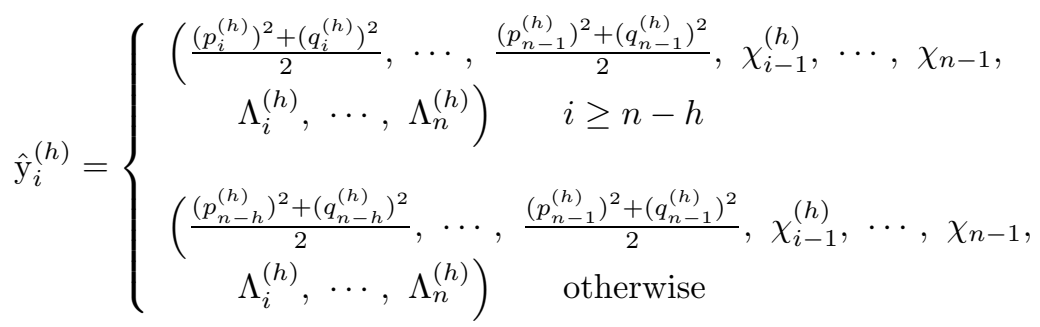

$$
\begin{aligned}
& \hat{\mathrm{x}}_{i}^{(h)}=\left\{\begin{array}{l}
\left(\kappa_{i}^{(h)}, \cdots, \kappa_{n-h-2}^{(h)}\right) \quad n \geq 4 \& 1 \leq h \leq n-3 \& 1 \leq i \leq n-h-2 \\
\emptyset \quad \text { otherwise }
\end{array}\right.
\end{aligned}
$$

and $\hat{\mathrm{y}}:=\hat{\mathrm{y}}_{1}, \hat{\mathrm{x}}:=\hat{\mathrm{x}}_{1}, f_{\mathfrak{s e c}, h}$ has the form

$$
\begin{aligned}
f_{\mathfrak{s e c}, h}\left(\mathrm{t}^{(h)}, \mathrm{z}^{(h)}, \mathrm{y}^{(h)}, \mathrm{x}^{(h)}\right) & =\mathrm{h}_{\mathfrak{s e c}, h}\left(\hat{\mathrm{y}}_{n-h}^{(h)}\right)+f_{\mathfrak{n o r m}, h}\left(\mathrm{t}^{(h)}, \hat{\mathrm{y}}^{(h)}, \hat{\mathrm{x}}^{(h)}\right) \\
& +f_{\mathfrak{e x p}, \mathfrak{s e c}, h}\left(\mathrm{t}^{(h)}, \mathrm{z}^{(h)}, \mathrm{y}^{(h)}, \mathrm{x}^{(h)}\right)
\end{aligned}
$$

with

$$
\begin{aligned}
& \mathrm{h}_{\mathfrak{s e c}}\left(\hat{\mathrm{y}}_{n-h}^{(h)}\right)=\sum_{i=n-h}^{n-1} \mathrm{~h}_{\mathfrak{s e c}}^{i}\left(\hat{\mathrm{y}}_{i}^{(h)}\right) \\
& f_{\mathfrak{n o r m}, h}\left(\mathrm{t}^{(h)}, \hat{\mathrm{y}}^{(h)}, \hat{\mathrm{x}}^{(h)}\right)=\sum_{i=1}^{n-h-1} f_{\mathfrak{n o r m}, h}^{i}\left(\mathrm{t}_{i}^{(h)}, \hat{\mathrm{y}}_{i}^{(h)}, \hat{\mathrm{x}}_{i}^{(h)}\right)
\end{aligned}
$$

where

3. the functions $\mathrm{h}_{\mathfrak{s e c}}^{i} f_{\mathfrak{n o r m}, h}^{i}$ may be decomposed as

$$
\begin{aligned}
& \mathrm{h}_{\mathfrak{s e c}}^{i}\left(\hat{\mathrm{y}}_{i}^{(h)}\right)=\overline{\mathrm{h}_{\mathfrak{s e c}}^{i}}\left(\hat{\mathrm{y}}_{i}^{(h)}\right)+\widetilde{\mathrm{h}_{\mathfrak{s e c}}^{i}} i\left(\hat{\mathrm{y}}_{i}^{(h)}\right) \\
& f_{\mathfrak{n o r m}, h}^{i}\left(\mathrm{t}_{i}^{(h)}, \hat{\mathrm{y}}_{i}^{(h)}, \hat{\mathrm{x}}_{i}^{(h)}\right)=\widetilde{f_{\mathfrak{n o r m}, h}^{i}}\left(\mathrm{t}_{i}^{(h)}, \hat{\mathrm{y}}_{i}^{(h)}, \hat{\mathrm{x}}_{i}^{(h)}\right)+\widetilde{f_{\mathfrak{n o r m}, h}^{i}\left(\mathrm{t}_{i}^{(h)}, \hat{\mathrm{y}}_{i}^{(h)}, \hat{\mathrm{x}}_{i}^{(h)}\right)}
\end{aligned}
$$

where

$$
\overline{f_{\mathfrak{n o r m}, h}^{i}}=\sum_{j=i+1}^{n} \Pi_{h}\left(\overline{f_{\mathcal{P}}^{i j}}{ }^{(\geq 2)} \circ \overline{\phi_{\mathfrak{i n t}}^{n-1}} \circ \cdots \circ \overline{\phi_{\mathfrak{i n t}}^{n-h}}\right)
$$


and $\overline{\mathrm{h}_{\mathfrak{s e c}}^{i}}, \overline{\phi_{\mathfrak{i n t}}^{i}}$ as in Lemma 3.4 . The functions $\widetilde{\mathrm{h}_{\mathfrak{s e c}, h}}, \widetilde{f_{\mathfrak{n o r m}, h},} f_{\mathfrak{e x p}, \mathfrak{s e c}, h}$ in (5.72) may be bounded as

$$
\begin{aligned}
& \widetilde{\mathrm{h}_{\mathfrak{s e c}, h}^{i} \mid} \leq \frac{1}{\mathfrak{c}_{h}} \max \left\{\mu \bar{K}\left(\frac{a_{n}}{a_{1}}\right)^{3 / 2} \frac{1}{a_{i+1}^{-}}, \quad \frac{\bar{K}^{\bar{\tau}+1} \sqrt{\alpha}}{\bar{\gamma}} \frac{\left(a_{i}^{+}\right)^{2}}{\left(a_{i+1}^{+}\right)^{3}}, \quad \frac{\varepsilon_{i+1}^{2}}{\theta_{i+1}} \frac{\left(a_{i}^{+}\right)^{2}}{\left(a_{i+1}^{-}\right)^{3}}\right\} \\
& \left|\widetilde{f_{\mathfrak{n o r m}, h}^{i}}\right| \leq \frac{1}{\mathfrak{c}_{h}} \max \left\{\mu \bar{K}\left(\frac{a_{n}}{a_{1}}\right)^{3 / 2} \frac{1}{a_{i+1}^{-}}, \quad \frac{\bar{K}^{\bar{\tau}+1} \sqrt{\alpha}}{\bar{\gamma}} \frac{\left(a_{i}^{+}\right)^{2}}{\left(a_{i+1}^{-}\right)^{3}}\right\} \\
& \left|f_{\mathfrak{e} \mathfrak{k} \mathfrak{p}, \mathfrak{s e c}, h}\right| \leq \frac{1}{\mathfrak{c}_{h}} \frac{\left(a_{n-1}^{+}\right)^{2}}{\left(a_{n}^{-}\right)^{3}} e^{-\mathfrak{c}_{h} \bar{K}}
\end{aligned}
$$

4. Defining

$$
\zeta^{(h)}:=\left(\frac{\left(p_{n-h}^{(h)}\right)^{2}+\left(q_{n-h}^{(h)}\right)^{2}}{2}, \cdots, \frac{\left(p_{n-1}^{(h)}\right)^{2}+\left(q_{n-1}^{(h)}\right)^{2}}{2}, \chi_{i-1}^{(h)}, \cdots, \chi_{n-1}\right)
$$

so that

for any $\Lambda_{n-h}^{(h)}, \cdots, \Lambda_{n}^{(h)}$, the map

$$
\hat{\mathrm{y}}_{n-h}^{(h)}=\left(\zeta^{(h)}, \Lambda_{n-h}^{(h)}, \cdots, \Lambda_{n}^{(h)}\right)
$$

$$
\zeta^{(h)} \rightarrow \omega_{\mathfrak{s e c}, h}:=\partial_{\zeta^{(h)}} \mathrm{h}_{\mathfrak{s e c}, h}\left(\zeta^{(h)}, \Lambda^{(h)}\right)
$$

is a diffeomorphism of $D_{\bar{r}} \times \mathrm{X}_{\bar{r}}$, with non-vanishing Jacobian matrix. The set $D_{\bar{r}}^{*} \times \mathrm{X}_{\bar{r}}^{*}$ consists of the subset of $D_{\bar{r}} \times \mathrm{X}_{\bar{r}}$ such that $\omega_{\mathfrak{f a s t}, \mathfrak{s e c}} \in \mathcal{D}_{\gamma_{\mathfrak{s e c}} ; \bar{\tau}}^{\bar{K}, \nu_{\mathfrak{s} \mathfrak{c}}}$, where, if $\nu_{j}, \gamma_{j}$ are as in (4.56),

$$
\nu_{\mathfrak{s e c}}:=\left(\nu_{n+1}, \cdots, \nu_{2 n-1}\right) \quad \gamma_{\mathfrak{s e c}}:=\left(\gamma_{n+1}, \cdots, \gamma_{2 n-1}\right) .
$$

We shall give the complete details of the proof of Proposition 5.2 along the following sections 5.2.1 5.2.4. In this section we just provide main ideas.

Scheme of Proof. The proof is by recursion. The $h^{\text {th }}$ step of this recursion starts with

$$
f_{\mathfrak{s e c}, h-1}=\mathrm{h}_{\mathfrak{s e c}, h-1}+f_{\mathfrak{n o r m}, h-1}+f_{\mathfrak{e x p}, \mathfrak{s e c}, h-1},
$$

where, for $h=1$

$$
\mathrm{h}_{\mathfrak{s e c}, 0} \equiv 0, \quad f_{\mathfrak{e r p}, \mathfrak{s e c}, 0} \equiv 0, \quad f_{\mathfrak{s e c}, 0}:=f_{\mathfrak{n o r m}, 0}:=f_{\mathfrak{f a s t}},
$$

while, for $n \geq 3$ and $h=2, \cdots, n-1$, we assume, inductively, that $\mathrm{h}_{\mathfrak{s e c}, h-1}, f_{\mathfrak{s e c}, h-1}$ and $f_{\mathfrak{e x p}, \mathfrak{s e c}, h-1}$ satisfy the theses of Proposition 5.2, with $h$ replaced by $(h-1)$.

The transformation $\phi_{\mathfrak{s e c}}^{n-h}$ conjugating $f_{\mathfrak{s e c}, h-1}$ to $f_{\mathfrak{s e c}, h}$ will be constructed as a product $\phi_{\mathfrak{s e c}}^{n-h}=\phi_{\mathfrak{i n t}}^{n-h} \circ \phi_{\mathfrak{n o r m}}^{n-h}$ of an "integrating" and a "normalizing" transformation.

Due to the bound on $f_{\mathfrak{e x p}, \mathfrak{s e c}, h-1}$, it is enough to focus on the truncation

$$
\widehat{f_{\mathfrak{s e c}, h-1}}:=\mathrm{h}_{\mathfrak{s e c}, h-1}+f_{\mathfrak{n o r m}, h-1}=\mathrm{h}_{\mathfrak{s e c}, h-1}+\sum_{i=1}^{n-h} f_{\mathfrak{n o r m}, h-1}^{i}\left(\mathrm{t}_{i}^{(h-1)}, \hat{\mathrm{y}}_{i}^{(h-1)}, \hat{\mathrm{x}}_{i}^{(h-1)}\right)
$$

of $f_{\mathfrak{s e c}, h-1}$. We split

$$
f_{\mathfrak{n o r m}, h-1}=f_{\mathfrak{n o r m}, h-1}^{n-h}\left(\mathrm{t}_{n-h}^{(h-1)}, \hat{\mathrm{y}}_{n-h}^{(h-1)}, \hat{\mathrm{x}}_{n-h}^{(h-1)}\right)+\sum_{i=1}^{n-h-1} f_{\mathfrak{n o r m}, h-1}^{i}\left(\mathrm{t}_{i}^{(h-1)}, \hat{\mathrm{y}}_{i}^{(h-1)}, \hat{\mathrm{x}}_{i}^{(h-1)}\right)
$$


and we distinguish two cases.

Case $n \geq 3, h=2, \cdots, n-1$. By the inductive assumption (see (5.71) with $h$ replaced by $(h-1))$, the function $f_{\mathfrak{n o r m}, h-1}^{n-h}$ depends only on

$$
\mathrm{t}_{n-h}^{(h-1)}=\left(\Theta_{n-h}^{(h-1)}, \vartheta_{n-h}^{(h-1)}\right) \text { and } \hat{\mathrm{y}}_{n-h}^{(h-1)}
$$

therefore is integrable. In Chapter 5.2.2, we shall construct a canonical, realanalytic change of coordinates

$$
\begin{aligned}
\phi_{\mathfrak{i n t}}^{n-h}: \quad & \mathfrak{D}_{\mathfrak{i n t}}^{h} \rightarrow \mathfrak{D}_{\mathfrak{s e c}}^{h-1} \\
& \left(\mathrm{t}_{*}^{(h)}, \mathrm{z}_{*}^{(h)}, \mathrm{y}_{*}^{(h)}, \mathrm{x}_{*}^{(h)}\right) \rightarrow\left(\mathrm{t}^{(h-1)}, \mathrm{z}^{(h-1)}, \mathrm{y}^{(h-1)}, \mathrm{x}^{(h-1)}\right)
\end{aligned}
$$

(5.79) $\mathfrak{D}_{\mathfrak{i n t}}^{h}:=\mathrm{T}_{\hat{\mathfrak{c}}_{h} \theta}^{h} \times B_{\hat{\mathfrak{c}}_{h} \varepsilon_{n-h}}^{2} \times B_{\hat{\mathfrak{c}}_{h} \varepsilon}^{*, h-1} \times \mathrm{X}_{\hat{\mathfrak{c}}_{h} \theta, \hat{\mathfrak{c}}_{h} \bar{r}}^{*, h-1} \times \mathrm{A}_{\hat{\mathfrak{c}}_{h} \widetilde{r}} \times \mathbb{T}_{\hat{\mathfrak{c}}_{h} s}^{n} \times \mathbb{T}_{\hat{\mathfrak{c}}_{h} s}^{n}$

such that

$$
f_{\mathfrak{n o r m}, h-1}^{n-h} \circ \phi_{\mathfrak{i n t}}^{n-h}=\mathrm{h}_{\mathfrak{s e c}}^{n-h}\left(\hat{\mathrm{y}}_{*, n-h}^{(h)}\right)
$$

depends only on $\hat{\mathrm{y}}_{*, n-h}^{(h)}$, where $\hat{\mathrm{y}}_{*, i}^{(h)}$ is defined analogously to $\hat{\mathrm{y}}_{i}^{(h)}$ in (5.71). Here,

$$
\left\{\begin{array} { r l } 
{ \mathrm { t } _ { * } ^ { ( h ) } } & { : = ( \Theta _ { * } ^ { ( h ) } , \vartheta _ { * } ^ { ( h ) } ) } \\
{ \mathrm { z } _ { * } ^ { ( h ) } } & { : = ( p _ { * } ^ { ( h ) } , q _ { * } ^ { ( h ) } ) } \\
{ \mathrm { y } _ { * } ^ { ( h ) } } & { : = ( \chi _ { * } ^ { ( h ) } , \Lambda _ { * } ^ { ( h ) } ) } \\
{ \mathrm { x } _ { * } ^ { ( h ) } } & { : = ( \kappa _ { * } ^ { ( h ) } , \ell _ { * } ^ { ( h ) } ) }
\end{array} \quad \left\{\begin{array}{l}
\mathrm{t}^{(h-1)}:=\left(\Theta^{(h-1)}, \vartheta^{(h-1)}\right) \\
\mathrm{z}^{(h-1)}:=\left(p^{(h-1)}, q^{(h-1)}\right) \\
\mathrm{y}^{(h-1)}:=\left(\chi^{(h-1)}, \Lambda^{(h-1)}\right) \\
\mathrm{x}^{(h-1)}:=\left(\kappa^{(h-1)}, \ell^{(h-1)}\right)
\end{array}\right.\right.
$$

are defined analogously to (5.70).

We shall construct $\phi_{\mathfrak{i n t}}^{n-h}$ in such a way that it involves only the coordinates

$$
\phi_{\mathfrak{i n t}}^{n-h}:\left(\mathrm{z}_{*, n-h}^{(h)}, \mathrm{y}_{*, n-h}^{(h)}, \mathrm{x}_{*, n-h}^{(h)}\right) \rightarrow\left(\mathrm{t}_{n-h}^{(h-1)}, \mathrm{z}_{n-h+1}^{(h-1)}, \mathrm{y}_{n-h}^{(h-1)}, \mathrm{x}_{n-h}^{(h-1)}\right)
$$

with

$$
\begin{aligned}
\mathrm{z}_{*, n-h}^{(h)} & :=\left(p_{*, n-h}^{(h)}, \cdots, p_{n-1}^{(h)}, q_{*, n-h}^{(h)}, \cdots, q_{n-1}^{(h)}\right) \\
\mathrm{y}_{*, n-h}^{(h)} & :=\left(\chi_{*, n-h-1}^{(h)}, \cdots, \chi_{*, n-1}, \Lambda_{*, n-h}^{(h)}, \cdots, \Lambda_{n}^{(h)}\right) \\
\mathrm{x}_{*, n-h}^{(h)} & :=\left(\kappa_{*, n-h-1}^{(h)}, \cdots, \kappa_{*, n-1}^{(h)}, \ell_{*, n-h}^{(h)}, \cdots, \ell_{n}^{(h)}\right) \\
\mathrm{t}_{n-h}^{(h-1)} & :=\left(\Theta_{n-h}^{(h-1)}, \vartheta_{n-h}^{(h-1)}\right) \\
\mathrm{z}_{n-h+1}^{(h-1)} & :=\left(p_{n-h+1}^{(h-1)}, \cdots, p_{n-1}^{(h-1)}, q_{n-h+1}^{(h-1)}, \cdots, q_{n-1}^{(h-1)}\right) \\
\mathrm{y}_{n-h}^{(h-1)} & :=\left(\chi_{n-h-1}^{(h-1)}, \cdots, \chi_{n-1}, \Lambda_{n-h}^{(h-1)}, \cdots, \Lambda_{n}^{(h-1)}\right) \\
\mathrm{x}_{n-h}^{(h-1)} & :=\left(\kappa_{n-h-1}^{(h-1)}, \cdots, \kappa_{n-1}^{(h-1)}, \ell_{n-h}^{(h-1)}, \cdots, \ell_{n}^{(h-1)}\right)
\end{aligned}
$$


and has the form

$$
\phi_{\mathfrak{i n t}}^{n-h}:\left\{\begin{array}{l}
\Theta_{n-h}^{(h-1)}=\mathrm{F}_{\mathfrak{i n t}}^{(h)}\left(p_{*, n-h}^{(h)}, q_{*, n-h}^{(h)}, \tilde{\mathrm{y}}_{*}^{(h)}\right) \\
\vartheta_{n-h}^{(h-1)}-\pi=\mathrm{G}_{\mathfrak{i n t}}^{(h)}\left(p_{*, n-h}^{(h)}, q_{*, n-h}^{(h)}, \tilde{\mathrm{y}}_{*}^{(h)}\right) \\
\hat{\mathrm{z}}_{j}^{(h-1)}=\hat{\mathrm{z}}_{*, j}^{(h)} e^{\mathrm{i} \psi_{\mathfrak{i n t}, j}^{(h)}\left(p_{*, n-h}^{(h)}, q_{*, n-h}^{(h)}, \tilde{\mathrm{y}}_{*}^{(h)}\right)} \\
\mathrm{y}_{n-h}^{(h-1)}=\mathrm{y}_{*, n-h}^{(h)} \\
\mathrm{x}_{n-h}^{(h-1)}=\mathrm{x}_{*, n-h}^{(h)}+\varphi_{\mathfrak{i n t}}^{(h)}\left(p_{*, n-h}^{(h)}, q_{*, n-h}^{(h)}, \tilde{\mathrm{y}}_{*}^{(h)}\right)
\end{array}\right.
$$

with $\mathrm{F}_{\mathfrak{i n \mathfrak { t }}}^{(h)}, \mathrm{G}_{\mathfrak{i n \mathfrak { t }}}^{(h)}$ odd, $\psi_{\mathfrak{i n t}, j}^{(h)}, \varphi_{\mathfrak{i n t}}^{(h)}$ even in $\left(p_{*, n-h}^{(h)}, q_{*, n-h}^{(h)}\right)$,

$$
\begin{aligned}
& \tilde{\mathrm{y}}_{*}^{(h)}:=\left(\frac{\left(p_{*, n-h+1}^{(h)}\right)^{2}+\left(q_{*, n-h+1}^{(h)}\right)^{2}}{2}, \cdots \frac{\left(p_{*, 1}^{(h)}\right)^{2}+\left(q_{*, 1}^{(h)}\right)^{2}}{2}, \mathrm{y}_{*, n-h}^{(h)}\right) \\
& \hat{\mathrm{z}}_{j}^{(h-1)}:=\left(p_{j}^{(h-1)}, q_{j}^{(h-1)}\right):=p_{j}^{(h-1)}+\mathrm{i} q_{j}^{(h-1)} \\
& \hat{\mathrm{z}}_{*, j}^{(h)}:=\left(p_{*, j}^{(h)}, q_{*, j}^{(h)}\right):=p_{*, j}^{(h)}+\mathrm{i} q_{*, j}^{(h)}
\end{aligned}
$$

with $j=n-h+1, \cdots, n-1$, for $n \geq 3, h \geq 2$ and $\mathrm{y}_{*, n-h}^{(h)}$ as in (5.81).

In particular, observe that $\phi_{\mathfrak{i n t}}^{n-h}$ enjoys the following properties:

- it is co-variant with the symmetry: if

$$
\phi_{\text {int }}^{n-h}\left(\mathrm{t}_{*}^{(h)}, \mathrm{z}_{*}^{(h)}, \mathrm{y}_{*}^{(h)}, \mathrm{x}_{*}^{(h)}\right)=\left(\mathrm{t}^{(h-1)}, \mathrm{z}^{(h-1)}, \mathrm{y}^{(h-1)}, \mathrm{x}^{(h-1)}\right),
$$

then

$$
\phi_{\mathfrak{i n t}}^{n-h}\left(-\mathrm{t}_{*}^{(h)},-\mathrm{z}_{*}^{(h)}, \mathrm{y}_{*}^{(h)}, \mathrm{x}_{*}^{(h)}\right)=\left(-\mathrm{t}^{(h-1)},-\mathrm{z}^{(h-1)}, \mathrm{y}^{(h-1)}, \mathrm{x}^{(h-1)}\right)
$$

- leaves the "actions"

$$
\tilde{\mathrm{y}}_{*}^{(h)}=\tilde{\mathrm{y}}^{(h-1)}
$$

unvaried, where $\tilde{\mathrm{y}}_{*}^{(h)}$ is as in (5.83), and

$$
\tilde{\mathrm{y}}^{(h-1)}:=\left(\frac{\left(p_{n-h+1}^{(h-1)}\right)^{2}+\left(q_{n-h+1}^{(h-1)}\right)^{2}}{2}, \cdots \frac{\left(p_{1}^{(h-1)}\right)^{2}+\left(q_{1}^{(h-1)}\right)^{2}}{2}, \mathrm{y}_{n-h}^{(h-1)}\right)
$$

is defined analogously;

- leaves the averages with respect to the x-coordinates unvaried. Namely, for any real-analytic function $g$ on $\mathfrak{D}_{\mathfrak{s e c}}^{h-1}$,

$$
\Pi_{\mathrm{x}_{*}^{(h)}}\left(g \circ \phi_{\mathfrak{i n t}}^{n-h}\right)=\left(\Pi_{\mathrm{x}^{(h-1)}} g\right) \circ \phi_{\mathfrak{i n t}}^{n-h} .
$$

Applying $\phi_{\mathfrak{i n t}}^{n-h}$ to $\widehat{f_{\mathfrak{s e c}, h-1}}$ in (5.78), we obtain

$$
\begin{aligned}
f_{\mathfrak{s e c}, \mathfrak{i n t}, h-1} & :=\widehat{f_{\mathfrak{s e c}, h-1}} \circ \phi_{\mathfrak{i n t}}^{n-h} \\
& =\mathrm{h}_{\mathfrak{s e c}, h-1}+\mathrm{h}_{\mathfrak{s e c}}^{n-h}+\sum_{i=1}^{n-h-1} f_{\mathfrak{n o r m}, \mathfrak{i n t}, h-1}^{i}\left(\mathrm{t}_{*, i}^{(h)}, \tilde{\hat{\mathrm{y}}}_{*, i}^{(h)}, \tilde{\hat{\mathrm{x}}}_{*, i}^{(h)}\right) \\
& =\sum_{i=n-h}^{n-1} \mathrm{~h}_{\mathfrak{s e c}, h}^{i}\left(\hat{\mathrm{y}}_{*, i}^{(h)}\right)+\sum_{i=1}^{n-h-1} f_{\mathfrak{n o r m}, \mathfrak{i n t}, h-1}^{i}\left(\mathrm{t}_{*, i}^{(h)}, \tilde{\hat{\mathrm{y}}}_{*, i}^{(h)}, \tilde{\hat{\mathrm{x}}}_{*, i}^{(h)}\right)
\end{aligned}
$$


with

$$
\mathrm{h}_{\mathfrak{s e c}, h}:=\mathrm{h}_{\mathfrak{s e c}, h-1}+\mathrm{h}_{\mathfrak{s e c}}^{n-h}, \quad f_{\mathfrak{n o r m}, \mathfrak{i n t}, h-1}^{i}:=f_{\mathfrak{n o r m}, h-1}^{i} \circ \phi_{\mathfrak{i n t}}^{n-h}
$$

and (as it follows from (5.71) with $h-1$ replacing $h$ and (5.82) $) f_{\mathfrak{n o r m}, \mathfrak{i n t}, h-1}^{i}$ depends only on the arguments

$$
\begin{aligned}
& \mathrm{t}_{*, i}^{(h)}:=\left(\Theta_{*, i}^{(h)}, \cdots, \Theta_{*, n-h-1}^{(h)}, \vartheta_{*, i}^{(h)}, \cdots, \vartheta_{*, n-h-1}^{(h)}\right) \\
& \tilde{\hat{\mathrm{y}}}_{*, i}^{(h)}:=\left(p_{*, n-h}^{(h)}, q_{*, n-h}^{(h)}, \frac{\left(p_{*, n-h+1}^{(h)}\right)^{2}+\left(q_{*, n-h+1}^{(h)}\right)^{2}}{2}, \cdots, \frac{\left(p_{*, n-1}^{(h)}\right)^{2}+\left(q_{*, n-1}^{(h)}\right)^{2}}{2},\right. \\
& \left.\chi_{*, i-1}^{(h)}, \cdots, \chi_{*, n-1}, \Lambda_{*, i}^{(h)}, \cdots, \Lambda_{*, n}^{(h)}\right) \\
& \tilde{\hat{\mathrm{x}}}_{*, i}^{(h)}:=\left\{\begin{array}{l}
\left(\kappa_{*, i}^{(h)}, \cdots, \kappa_{*, n-h-1}^{(h)}\right) \quad n \geq 4 \& 1 \leq h-1 \leq n-3 \\
\emptyset \quad \text { otherwise }
\end{array}\right.
\end{aligned}
$$

The next step will be to retain the dependence on $\left(p_{n-h}^{(h)}, q_{n-h}^{(h)}\right)$ only via $\frac{\left(p_{n-h}^{(h)}\right)^{2}+\left(q_{n-h}^{(h)}\right)^{2}}{2}$ and, for $h<n-1$, to to eliminate from $f_{\mathfrak{s e c}, \mathfrak{i n t}, h-1}$ the dependence upon the angle $k_{*, n-h-1}^{(h)}$, up to an exponential remainder. Namely, we look for another canonical, real-analytic change of coordinates

$$
\begin{array}{ll}
\phi_{\mathfrak{n o r m}}^{n-h}: & \mathfrak{D}_{\mathfrak{s e c}}^{h} \rightarrow \mathfrak{D}_{\mathfrak{i n t}}^{h} \\
& \left(\mathrm{t}^{(h)}, \mathrm{z}^{(h)}, \mathrm{y}^{(h)}, \mathrm{x}^{(h)}\right) \rightarrow\left(\mathrm{t}_{*}^{(h)}, \mathrm{z}_{*}^{(h)}, \mathrm{y}_{*}^{(h)}, \mathrm{x}_{*}^{(h)}\right)
\end{array}
$$

so as to conjugate $f_{\mathfrak{s e c}, \text { int }, h-1}$ to a new Hamiltonian

$$
\widehat{f_{\mathfrak{s e c}, h}}:=f_{\mathfrak{s e c}, \mathfrak{i n t}, h-1} \circ \phi_{\mathfrak{n o r m}}^{n-h}=\mathrm{h}_{\mathfrak{s e c}, h}+\sum_{i=1}^{n-h-1} f_{\mathfrak{n o r m}, h}^{i}\left(\mathrm{t}_{i}^{(h)}, \hat{\mathrm{y}}_{i}^{(h)}, \hat{\mathrm{x}}_{i}^{(h)}\right)+\widehat{f_{\mathfrak{e x p}, \mathfrak{s e c}, h}}
$$

where $f_{\mathfrak{n o r m}, h}^{i}$ and $\widehat{f_{\mathfrak{e r p}, \mathfrak{s e c}, h}}$ satisfy (5.74)-(5.76). We choose $\mathfrak{D}_{\mathfrak{s e c}}^{h}$ as the subset of $\mathfrak{D}_{\mathfrak{i n t}}^{h}$ where the map

$$
\omega_{\mathfrak{s e c}, h}:= \begin{cases}\frac{\partial_{\left(p_{n-h}^{(h)}\right)^{2}+\left(q_{n-h}^{(h)}\right)^{2}}^{2}, \chi_{n-h-1}^{(h)} \mathrm{h}_{\mathfrak{s e c}, h}}{2} h=2, \cdots, n-2 \& n \geq 4 \\ \partial_{\frac{\left(p_{1}^{(n-1)}\right)^{2}+\left(q_{1}^{(n-1)}\right)^{2}}{2}} \mathrm{~h}_{\mathfrak{s e c}, n-1} \quad h=n-1\end{cases}
$$

does not verify resonances up to order $\bar{K}$, and next we apply a suitable normal form theory (Proposition D.1). We shall choose $\phi_{\mathfrak{n o r m}}^{n-h}$ in such a way that

- it is co-variant with the symmetry: if

$$
\phi_{\mathfrak{n o r m}}^{n-h}\left(\mathrm{t}^{(h)}, \mathrm{z}^{(h)}, \mathrm{y}^{(h)}, \mathrm{x}^{(h)}\right)=\left(\mathrm{t}_{*}^{(h)}, \mathrm{z}_{*}^{(h)}, \mathrm{y}_{*}^{(h)}, \mathrm{x}_{*}^{(h)}\right),
$$

then

$$
\phi_{\mathfrak{n o r m}}^{n-h}\left(-\mathrm{t}^{(h)},-\mathrm{z}^{(h)}, \mathrm{y}^{(h)}, \mathrm{x}^{(h)}\right)=\left(-\mathrm{t}_{*}^{(h)},-\mathrm{z}_{*}^{(h)}, \mathrm{y}_{*}^{(h)}, \mathrm{x}_{*}^{(h)}\right),
$$

- leaves the "actions"

$$
\mathrm{y}_{*, n-h}^{(h)}=\mathrm{y}_{n-h}^{(h)}
$$


unvaried, where

$$
\begin{aligned}
\mathrm{y}_{n-h}^{(h)}:= & \left(\frac{\left(p_{n-h+1}^{(h)}\right)^{2}+\left(q_{n-h+1}^{(h)}\right)^{2}}{2}, \cdots \frac{\left(p_{1}^{(h)}\right)^{2}+\left(q_{1}^{(h)}\right)^{2}}{2}, \chi_{n-h}^{(h)}, \cdots, \chi_{n-1}^{(h)},\right. \\
& \left.\Lambda_{1}^{(h)}, \cdots, \Lambda_{n}^{(h)}\right) ; \\
\mathrm{y}_{*, n-h}^{(h)}:= & \left(\frac{\left(p_{*, n-h+1}^{(h)}\right)^{2}+\left(q_{*, n-h+1}^{(h)}\right)^{2}}{2}, \cdots \frac{\left(p_{*, 1}^{(h)}\right)^{2}+\left(q_{*, 1}^{(h)}\right)^{2}}{2}, \chi_{*, n-h}^{(h)}, \cdots, \chi_{*, n-1}^{(h)},\right. \\
5.89) \quad & \left.\Lambda_{*, 1}^{(h)}, \cdots, \Lambda_{*, n}^{(h)}\right) ;
\end{aligned}
$$

- verifies

$$
\Pi_{\mathrm{z}_{*, n-h+1}^{(h)}, \mathrm{x}_{*, n-h+1}^{(h)}}\left(g \circ \phi_{\mathfrak{n o r m}}^{n-h}\right)=\left(\Pi_{\mathbf{z}_{n-h+1}^{(h)}, \mathrm{x}_{n-h+1}^{(h)}} g\right) \circ \phi_{\mathfrak{n o r m}}^{n-h} .
$$

The thesis of Proposition 5.2 at rank $h$ follows, with

$$
f_{\mathfrak{s e c}, h}:=\widehat{f_{\mathfrak{s e c}, h}}+f_{\mathfrak{e x p}, \mathfrak{s e c}, h-1} \circ \phi_{\mathfrak{s e c}}^{n-h}, f_{\mathfrak{e x p}, \mathfrak{s e c}, h}:=\widehat{f_{\mathfrak{e x p}, \mathfrak{s e c}, h}}+f_{\mathfrak{e x p}, \mathfrak{s e c}, h-1} \circ \phi_{\mathfrak{s e c}}^{n-h} .
$$

Case $h=1$. The proof of this case uses similar ideas as the proof of the case $2 \leq h \leq n-1$ for $n \geq 3$. However, due to subtle differences between the two cases (compare, e.g., the inductive assumption on $f_{\mathfrak{n o r m}, h-1}^{n-h}$ in (5.71) for $h \geq 2$ with Eq. (5.91); the definition of $\mathrm{h}_{\mathfrak{s e c}}^{n-h}, \phi_{\mathfrak{i n t}}^{n-h}$ for $h \geq 2$ in (5.80), with the definition of $\mathrm{h}_{\mathfrak{s e c}}^{n-1}$, $\phi_{\mathfrak{i n t}}^{n-1}$ in (5.93) and (5.96) ), for sake of precision, we briefly discuss also this case.

Let $f_{\mathfrak{s e c}, 0}$ be as in (5.77). In view of (5.60) and (5.61), we can split

$$
f_{\mathfrak{s e c}, 0}={\overline{f_{\mathcal{P}}^{n-1, n}}}^{(2)}+{\overline{f_{\mathcal{P}}^{n-1}}}^{(\geq 3)}+\widetilde{f_{\mathfrak{f a s t}}^{n-1}}+\sum_{i=1}^{n-2} f_{\mathfrak{f a} \mathfrak{s t}}^{i}
$$

where

$$
{\overline{f_{\mathcal{P}}^{n-1}}}^{(\geq 3)}:={\overline{f_{\mathcal{P}}^{n-1}}}^{(\geq 2)}-{\overline{f_{\mathcal{P}}^{n-1}}}^{(2)}
$$

and the summand appears only when $n \geq 3$. As for ${\overline{f_{\mathcal{P}}^{n-1, n}}}^{(2)}$, by Lemmata 3.4 (see also Lemma 5.2), we find a domain $\overline{\mathfrak{B}_{n-1}}$ (defined in Eq. (5.97) below), a real-analytic and canonical transformation

$$
\begin{aligned}
\phi_{\mathfrak{i n t}}^{n-1}:\left(\mathrm{z}_{*, n-1}^{(1)}, \mathrm{y}_{*, n-1}^{(1)}, \mathrm{x}_{*, n-1}^{(1)}\right) & \in \overline{\mathfrak{B}_{n-1}} \rightarrow\left(\mathrm{z}_{n-1}^{(0)}, \mathrm{y}_{n-1}^{(0)}, \mathrm{x}_{n-1}^{(0)}\right) \\
& \in \overline{\mathfrak{D}_{n-1}}:=\phi_{\mathfrak{i n \mathfrak { t }}}^{n-1}\left(\overline{\mathfrak{B}_{n-1}}\right)
\end{aligned}
$$

of the form (5.82), with $h=1$ (but neglecting the coordinates $\hat{\mathrm{z}}_{j}^{(0)}, \hat{\mathrm{z}}_{j, *}^{(1)}$ ) such that

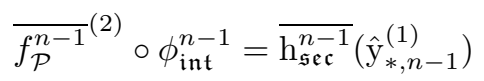

depends only on

$$
\hat{\mathrm{y}}_{*, n-1}^{(1)}=\left(\frac{\left(p_{*, n-1}^{(1)}\right)^{2}+\left(q_{*, n-1}^{(1)}\right)^{2}}{2}, \chi_{*, n-2}^{(1)}, \chi_{*, n-1}^{(1)}, \Lambda_{*, n-1}^{(1)}, \Lambda_{*, n}^{(1)}\right) .
$$

In (5.92), we have let

$$
\left\{\begin{array} { l } 
{ \mathrm { z } _ { * , n - 1 } ^ { ( 1 ) } : = ( p _ { * , n - 1 } ^ { ( 1 ) } , q _ { * , n - 1 } ^ { ( 1 ) } ) } \\
{ \mathrm { y } _ { * , n - 1 } ^ { ( 1 ) } : = ( \chi _ { * , n - 2 } ^ { ( 1 ) } , \chi _ { * , n - 1 } ^ { ( 1 ) } , \Lambda _ { * , n - 1 } ^ { ( 1 ) } , \Lambda _ { * , n } ^ { ( 1 ) } ) } \\
{ \mathrm { x } _ { * , n - 1 } ^ { ( 1 ) } : = ( \kappa _ { * , n - 2 } ^ { ( 1 ) } , \kappa _ { * , n - 1 } ^ { ( 1 ) } ) }
\end{array} \quad \left\{\begin{array}{l}
\mathrm{t}_{n-1}^{(0)}:=\left(\Theta_{n-1}^{(0)}, \vartheta_{n-1}^{(0)}\right) \\
\mathrm{y}_{n-1}^{(0)}:=\left(\chi_{n-2}^{(0)}, \chi_{n-1}^{(0)}, \Lambda_{n-1}^{(0)}, \Lambda_{n}^{(0)}\right) \\
\mathrm{x}_{n-1}^{(0)}:=\left(\kappa_{n-2}^{(0)}, \kappa_{n-1}^{(0)}\right) .
\end{array}\right.\right.
$$


We let

$$
\left\{\begin{array} { r l } 
{ \mathrm { t } ^ { ( 0 ) } } & { : = ( \Theta ^ { ( 0 ) } , \vartheta ^ { ( 0 ) } ) } \\
{ \mathrm { y } ^ { ( 0 ) } } & { : = ( \chi ^ { ( 0 ) } , \Lambda ^ { ( 0 ) } ) } \\
{ \mathrm { x } ^ { ( 0 ) } } & { : = ( \kappa ^ { ( 0 ) } , \ell ^ { ( 0 ) } ) }
\end{array} \quad \left\{\begin{array}{l}
\mathrm{t}_{*}^{(1)}:=\left(\Theta_{*}^{(1)}, \vartheta_{*}^{(1)}\right) \\
\mathrm{z}_{*}^{(1)}:=\left(p_{*}^{(1)}, q_{*}^{(1)}\right) \\
\mathrm{y}_{*}^{(1)}:=\left(\chi_{*}^{(1)}, \Lambda_{*}^{(1)}\right) \\
\mathrm{x}_{*}^{(1)}:=\left(\kappa_{*}^{(1)}, \ell_{*}^{(1)}\right)
\end{array}\right.\right.
$$

analogously to (5.70), with $h=0,1$, and then we regard the map in (5.92) as a map

$$
\phi_{\mathfrak{i n t}}^{n-1}:\left(\mathrm{t}_{*}^{(1)}, \mathrm{z}_{*}^{(1)}, \mathrm{y}_{*}^{(1)}, \mathrm{x}_{*}^{(1)}\right) \in \mathfrak{D}_{\mathfrak{i n t}}^{1} \rightarrow\left(\mathrm{t}^{(0)}, \mathrm{y}^{(0)}, \mathrm{x}^{(0)}\right)
$$

on the set

$$
\mathfrak{D}_{\mathfrak{i n t}}^{1}:=\left\{\left(\mathrm{t}_{*}^{(1)}, \mathrm{z}_{*}^{(1)}, \mathrm{y}_{*}^{(1)}, \mathrm{x}_{*}^{(1)}\right): \quad\left(\mathrm{z}_{*, n-1}^{(1)}, \mathrm{y}_{*, n-1}^{(1)}, \mathrm{x}_{*, n-1}^{(1)}\right) \in \overline{\mathfrak{B}_{n-1}}\right\}
$$

where $\phi_{\mathfrak{i n t}}^{n-1}$ is defined on the extra-coordinates via the identity. $\mathfrak{D}_{\mathfrak{i n t}}^{1}$ has the form in (5.79), with $h=1$. Applying this extension to $f_{\mathfrak{s e c}, 0}$ in (5.91) we obtain

$$
f_{\mathfrak{s e c}, \mathfrak{i n t}, 0}:=f_{\mathfrak{s e c}, 0} \circ \overline{\phi_{\mathfrak{i n t}}^{n-1}}=\overline{\mathrm{h}_{\mathfrak{s e c}}^{n-1}}\left(\hat{\mathrm{y}}_{*, n-1}^{(1)}\right)+\sum_{i=1}^{n-1} f_{\mathfrak{n o r m}, \mathfrak{i n t}, 0}^{i}\left(\mathrm{t}_{*, i}^{(1)}, \tilde{\hat{\mathrm{y}}}_{*, i}^{(1)}, \tilde{\hat{\mathrm{x}}}_{*, i}^{(1)}\right)
$$

where

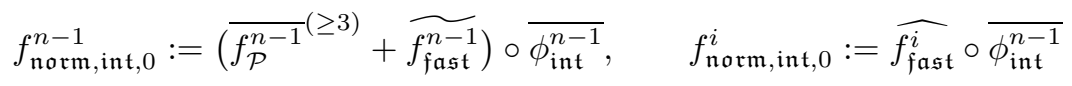

and, as a consequence of (5.61) and of (5.82), with $h=1, f_{\mathfrak{n o r m}, i n \mathfrak{r}, 0}^{i}$ depends only on the arguments

$$
\begin{aligned}
& \mathrm{t}_{*, i}^{(1)}:=\left(\Theta_{*, i}^{(1)}, \cdots, \Theta_{*, n-2}^{(1)}, \vartheta_{*, i}^{(1)}, \cdots, \vartheta_{*, n-2}^{(1)},\right) \\
& \tilde{\hat{\mathrm{y}}}_{*, i}^{(1)}::=\left(p_{*, n-1}^{(1)}, q_{*, n-1}^{(1)}, \chi_{*, i-1}^{(1)}, \cdots, \chi_{*, n-1}, \Lambda_{*, i}^{(1)}, \cdots, \Lambda_{*, n}^{(1)}\right) \\
& \tilde{\hat{\mathrm{x}}}_{*, i}^{(1)}:=\left(\kappa_{*, i}^{(1)}, \cdots, \kappa_{*, n-1}^{(1)}\right) .
\end{aligned}
$$

Note, in particular, that $f_{\mathfrak{n o r m}, \mathfrak{i n t}, 0}^{n-1}$ is a function of

$$
\left(\mathrm{t}_{*, n-1}, \mathrm{y}_{*, n-1}, \mathrm{x}_{*, n-1}\right)=\left(p_{* n-1}^{(1)}, q_{* n-1}^{(1)}, \chi_{*, n-2}, \chi_{*, n-1}, \Lambda_{*, n-1}, \Lambda_{*, n}, \kappa_{* n-1}\right) .
$$

In view of the fact that $\overline{h_{\mathfrak{s e c}}^{n-1}}$ depends on the actions in (5.94), we aim to eliminate from $f_{\mathfrak{s e c}, \mathfrak{i n t}, 0}$ the dependence on the following angles

$$
\left\{\begin{array}{lll}
\kappa_{*, 1} & \text { if } & n=2 \\
\kappa_{*, n-2}, \kappa_{*, n-1} & \text { if } & n \geq 3
\end{array}\right.
$$

and to retain the dependence on $\left(p_{*, n-1}^{(1)}, q_{*, n-1}^{(1)}\right)$ only via $\frac{\left(p_{*, n-1}^{(1)}\right)^{2}+\left(q_{*, n-1}^{(1)}\right)^{2}}{2}$. Then we choose a domain $\mathfrak{D}_{\mathfrak{s e c}}^{1} \subset \mathfrak{D}_{\mathfrak{i n t}}^{1}$ as in (5.69) where the frequency

$$
\omega_{\mathfrak{s e c}, 1}:= \begin{cases}\frac{\partial_{\left.p_{*, n-1}^{(1)}\right)^{2}+\left(q_{*, n-1}^{(1)}\right)^{2}}}{2}, \chi_{*, n-1}^{(1)} \overline{\mathrm{h}_{\mathfrak{s e c}}^{n-1}} & n=2 \\ \partial_{\frac{\left.p_{*, n-1}^{(1)}\right)^{2}+\left(q_{*, n-1}^{(1)}\right)^{2}}{2}, \chi_{*, n-2}^{(1)}, \chi_{*, n-1}^{(1)} \overline{\mathrm{h}_{\mathfrak{s e c}}^{n-1}}} & n \geq 3\end{cases}
$$


is non-resonant up to the order $\bar{K}$ and on this domain we construct a real-analytic transformation $\phi_{\mathfrak{n o r m}}^{n-1}$ as in (5.86) which conjugates $\overline{\mathrm{f}_{\mathfrak{s e c}, 1}}$ to a Hamiltonian

$f_{\mathfrak{s e c}, 1}:=f_{\mathfrak{s e c}, \mathfrak{i n t}, 0} \circ \phi_{\mathfrak{n o r m}}^{n-1}=\overline{\mathrm{h}_{\mathfrak{s e c}}^{n-1}}\left(\hat{\mathrm{y}}_{n-1}^{(1)}\right)+\sum_{i=1}^{n-1} f_{\mathfrak{n o r m}, 1}^{i}\left(\mathrm{t}_{i}^{(1)}, \hat{\mathrm{y}}_{i}^{(1)}, \hat{\mathrm{x}}_{i}^{(1)}\right)+f_{\mathfrak{e r p}, \mathfrak{s e c}, 1}$

Now, since (as it follows from (5.95) $), f_{\mathfrak{n o r m}, 1}^{n-1}$ is actually a function of $\hat{y}_{n-1}^{(1)}$ only, this step is proved, with

$$
\mathrm{h}_{\mathfrak{s e c}}^{n-1}\left(\hat{\mathrm{y}}_{n-1}^{(1)}\right):=\overline{\mathrm{h}_{\mathfrak{s e c}}^{n-1}}\left(\hat{\mathrm{y}}_{n-1}^{(1)}\right)+f_{\mathfrak{n o r m}, 1}^{n-1}\left(\hat{\mathrm{y}}_{n-1}^{(1)}\right)
$$

5.2.1. Construction of $\phi_{\mathfrak{i n t}}^{n-1}$. The following lemma completes Lemma 3.4. In particular, it provides the transformation $\phi_{\mathfrak{i n t}}^{n-1}=\overline{\phi_{\mathfrak{i n t}}^{n-1}}$ in (5.93).

Lemma 5.2. Let $i=1, \cdots, n-1$. Let $\mathcal{A}, \mathrm{X}, \theta$ in (4.46) be chosen in such a way that

$$
\begin{aligned}
& \inf _{\mathfrak{D}_{\mathcal{P}}}|g|>0, \quad \sup _{\mathfrak{D}_{\mathcal{P}}}|\arg g|<\frac{\pi}{4} \\
& \forall g \in\left\{\chi_{i-1}, \quad \chi_{i}, \quad \chi_{i-1}+\chi_{i}, \quad 5 \chi_{i-1} \Lambda_{i}^{2}-\left(\chi_{i-1}-\chi_{i}\right)^{2}\left(4 \chi_{i-1}-\chi_{i}\right)\right\}
\end{aligned}
$$

Then, the domains $\overline{\mathfrak{B}_{i}}$ in (3.42), the functions $\overline{\mathrm{h}_{\mathfrak{s e c}}^{i}}$ and the transformations $\overline{\phi_{\mathfrak{i n t}}^{i}}$ can be taken as follows

$$
\begin{aligned}
& \overline{\mathfrak{B}_{i}}=\left\{\begin{array}{l}
B_{\varepsilon_{i}}^{2} \times \mathcal{A}_{\bar{\theta}^{i}}^{i} \times \chi_{\bar{\theta}^{i}}^{i} \times \mathbb{T}_{\bar{s}^{i}}^{4} \quad i=n-1 \\
B_{\varepsilon_{i}}^{2} \times \mathcal{A}_{\bar{\theta}^{i}}^{i} \times \chi_{\bar{\theta}^{i}}^{i} \times \mathbb{T}_{\bar{s}^{i}}^{5} \quad i=1, \cdots, n-2 \quad \& \quad n \geq 3
\end{array}\right. \\
& \overline{\phi_{\mathfrak{i n t}}^{i}}:\left\{\begin{array}{l}
\Theta_{i}=\frac{p_{i}}{\beta_{i}}+f_{i}\left(p_{i}, q_{i}, \mathrm{y}_{i}^{*}\right) \\
\vartheta_{i}-\pi=\beta_{i} q_{i}+g_{i}\left(p_{i}, q_{i}, \mathrm{y}_{i}^{*}\right) \\
\mathrm{y}_{i}=\mathrm{y}_{i}^{*} \\
\mathrm{x}_{i}=\mathrm{x}_{i}^{*}+\varphi_{i}\left(p_{i}, q_{i}, \mathrm{y}_{i}^{*}\right)
\end{array}\right. \\
&(5.98) \overline{\mathrm{h}_{\mathfrak{s e c}}^{i}}=\mathcal{A}_{i}\left[\mathrm{E}_{i}+\Omega_{i} \frac{p_{i}^{2}+q_{i}^{2}}{2}+\tau_{i}\left(\frac{p_{i}^{2}+q_{i}^{2}}{2}\right)^{2}+\mathrm{O}\left(p_{i}, q_{i}\right)^{6}\right]
\end{aligned}
$$


where $\mathrm{X}_{\bar{\theta}^{i}}^{i} \times \mathrm{A}_{\bar{\theta}^{i}}^{i}$ denote the projection of the set $\mathrm{X}_{\bar{\theta}} \times \mathrm{A}_{\bar{\theta}}$ over the coordinates $\mathrm{y}_{i}$ in (3.43), $\bar{\theta}:=\theta / 2, \bar{s}:=s / 2, f_{i}, g_{i}$ are $\mathrm{O}\left(p_{i}, q_{i}\right)^{3}$, odd in $\left(p_{i}, q_{i}\right), \varphi_{i}$ is $\mathrm{O}\left(p_{i}, q_{i}\right)^{2}$, and

$$
\begin{aligned}
\varepsilon_{i} & =\mathfrak{c}_{i} \sqrt{\theta_{i}} \\
\beta_{i} & :=\sqrt[4]{\frac{5 \chi_{i-1} \Lambda_{i}^{2}-\left(\chi_{i-1}-\chi_{i}\right)^{2}\left(4 \chi_{i-1}-\chi_{i}\right)}{\chi_{i-1}^{2} \chi_{i}^{2}\left(\chi_{i-1}+\chi_{i}\right)}} \\
\mathcal{A}_{i} & :=m_{i} m_{i+1} \frac{a_{i}^{2}}{4 a_{i+1}^{3}} \\
\mathrm{E}_{i} & :=-\frac{\Lambda_{i+1}^{3}}{2\left(\chi_{i}-\chi_{i+1}\right)^{3}}\left(5-3 \frac{\left(\chi_{i-1}-\chi_{i}\right)^{2}}{\Lambda_{i}^{2}}\right) \\
\Omega_{i} & :=\frac{3 \Lambda_{i+1}^{3}}{\chi_{i} \Lambda_{i}^{2}\left(\chi_{i}-\chi_{i+1}\right)^{3}} \sqrt{\left(5 \chi_{i-1} \Lambda_{i}^{2}-\left(\chi_{i-1}-\chi_{i}\right)^{2}\left(4 \chi_{i-1}-\chi_{i}\right)\right)\left(\chi_{i-1}+\chi_{i}\right)} \\
\tau_{i} & :=\frac{\Lambda_{i+1}^{3}}{\chi_{i}^{2}\left(\chi_{i}-\chi_{i+1}\right)^{3}}\left[-\frac{9}{16} \frac{\left(\chi_{i-1}-\chi_{i}\right)^{2}\left(3 \chi_{i-1}-\chi_{i}\right)\left(5 \chi_{i-1}+\chi_{i}\right)}{\chi_{i-1}^{3} \chi_{i} \Lambda_{i}^{2} \beta_{i}^{4}}\right. \\
(5.99) & \left.-\frac{3}{8} \frac{2 \chi_{i-1}^{3}+9 \chi_{i-1}^{2} \chi_{i}+2 \chi_{i-1} \chi_{i}^{2}+\chi_{i}^{3}}{\chi_{i-1} \Lambda_{i}^{2}}-\frac{3}{16} \frac{\chi_{i-1} \chi_{i}^{2}}{\Lambda_{i}^{2}}\left(4 \chi_{i-1}+\chi_{i}\right) \beta_{i}^{4}\right]
\end{aligned}
$$

with $\chi_{n} \equiv 0, \overline{\mathfrak{c}}_{i}$ depending at most on the ratios $a_{i}^{+} / a_{i}^{-}$, the masses $m_{1}, \cdots, m_{n}$ and, as usual, $\sqrt[m]{z}$ denoting the principal determination of the $m^{\text {th }}$ root of a complex number $z$.

Proof. Since the formula for $\overline{\overline{f_{\mathcal{P}}^{n-1, n}}}$ coincides with the one for $\overline{f_{\mathcal{P}}^{n-1, n}}$ taking $\chi_{n} \equiv 0$, we shall only work on the terms $\overline{\overline{f_{\mathcal{P}}^{i, i+1}}}$, s.

Let $\mathrm{y}_{i}$ be as in (3.43), and let

$$
\mathfrak{D}_{i}: \quad\left(\Theta_{i}, \vartheta_{i}\right) \in \mathrm{T}_{\Theta_{i}^{+}, \vartheta_{i}^{+}}^{i} \quad \mathrm{y}_{i} \in \mathcal{A}_{\theta_{i}}^{i} \times \mathrm{X}_{\theta_{i}}^{i} \quad \mathrm{x}_{i} \in \mathbb{T}_{s}^{m_{i}}
$$

where $\mathrm{T}_{\Theta_{i}^{+}, \vartheta_{i}^{+}}^{i}$ is the projection of $\mathrm{T}_{\Theta^{+}, \vartheta^{+}}$over the coordinates $\left(\Theta_{i}, \vartheta_{i}\right)$, while $m_{i}$ is 4 or 5 , accordingly to (5.98). We shall obtain the transformation $\overline{\phi_{\mathfrak{i n t}}^{i}}$ in (3.42) as a product $\overline{\phi_{\mathfrak{i n t}}^{i}}=\overline{\phi_{\mathfrak{d} \mathfrak{i a g}}^{i}} \circ \overline{\phi_{\mathfrak{b i r}}^{i}}$, where $\overline{\phi_{\mathfrak{d} \mathfrak{i a g}}^{i}}$ and $\overline{\phi_{\mathfrak{b i r}}^{i}}$ are described below.

A Taylor expansion of $\overline{\overline{f_{\mathcal{P}}^{i, i+1}}}$ around $\left(\Theta_{i}, \vartheta_{i}\right)=(0, \pi)$ gives

$$
\overline{\overline{f_{\mathcal{P}}^{i, i+1}}}=\mathcal{A}_{i}\left[\mathrm{E}_{i}+\Omega_{i} \frac{\beta_{i}^{2} \Theta_{i}^{2}+\frac{\left(\vartheta_{i}-\pi\right)^{2}}{\beta_{i}^{2}}}{2}+\mathcal{R}_{i}\right]
$$

where $\mathcal{A}_{i}, \mathrm{E}_{i}, \beta_{i}, \Omega_{i}$ are as in (5.99). Note that $\beta_{i}, \Omega_{i}$ are well defined under the assumption (5.97). The expansion in (5.101) shows that $\left(\Theta_{i}, \vartheta_{i}\right)=(0, \pi)$ is an elliptic equilibrium point for $\overline{\overline{f_{\mathcal{P}}^{i, i+1}}}$. The remainder $\mathcal{R}_{i}$ is given by

$$
\begin{aligned}
\mathcal{R}_{i} & =\mathrm{F}\left[-\frac{3}{2} \frac{4 \Theta_{i}^{2}-\chi_{i}^{2}}{\Lambda_{i}^{2}}\left(\frac{\left(\chi_{i}^{2}-\chi_{i-1}^{2}\right)^{2}}{\left(\sqrt{\chi_{i}^{2}-\Theta_{i}^{2}}+\sqrt{\chi_{i-1}^{2}-\Theta_{i}^{2}}\right)^{2}}\right.\right. \\
& \left.\left.+2 \sqrt{\left(\chi_{i}^{2}-\Theta_{i}^{2}\right)\left(\chi_{i-1}^{2}-\Theta_{i}^{2}\right)}\left(1+\cos \vartheta_{i}\right)\right)+\frac{1}{2} \frac{\left(\chi_{i}^{2}-\Theta_{i}^{2}\right)\left(\chi_{i-1}^{2}-\Theta_{i}^{2}\right)}{\Lambda_{i}^{2}} \sin ^{2} \vartheta_{i}\right]
\end{aligned}
$$


where the symbol $\mathrm{F}$ on the left means that only terms of the fourth order in $\left(\Theta_{i}, \vartheta_{i}-\right.$ $\pi)$ have to be included. The lower order expansion of $\mathcal{R}_{i}$ is

$$
\mathcal{R}_{i}=\tau_{1, i} \Theta_{i}^{4}+\tau_{2, i}\left(\vartheta_{i}-\pi\right)^{2} \Theta_{i}^{2}+\tau_{3, i}\left(\vartheta_{i}-\pi\right)^{4}+\mathrm{O}\left(\Theta_{i}, \vartheta_{i}-\pi\right)^{6}
$$

with

$$
\begin{aligned}
\tau_{1, i} & :=\tau_{1}\left(\mathrm{y}_{i}\right):=-\frac{3\left(\chi_{i-1}-\chi_{i}\right)^{2}\left(3 \chi_{i-1}-\chi_{i}\right)\left(5 \chi_{i-1}+\chi_{i}\right)}{8 \chi_{i-1}^{3} \chi_{i} \Lambda_{i}^{2}} \\
\tau_{2, i} & :=\tau_{2}\left(\mathrm{y}_{i}\right):=-\frac{3\left(2 \chi_{i-1}^{3}+9 \chi_{i-1}^{2} \chi_{i}+2 \chi_{i-1} \chi_{i}^{2}+\chi_{i}^{3}\right)}{4 \chi_{i-1} \Lambda_{i}^{2}} \\
\tau_{3, i} & :=\tau_{3}\left(\mathrm{y}_{i}\right):=-\frac{\chi_{i-1} \chi_{i}^{2}}{8 \Lambda_{i}^{2}}\left(4 \chi_{i-1}+\chi_{i}\right) .
\end{aligned}
$$

We introduce the generating function

$$
S_{\mathfrak{d i a g}, i}\left(\tilde{p}_{i}, \tilde{\mathrm{y}}_{i}, \vartheta_{i}, \mathrm{x}_{i}\right)=\frac{\tilde{p}_{i}\left(\vartheta_{i}-\pi\right)}{\tilde{\beta}_{i}}+\tilde{\mathrm{y}}_{i} \mathrm{x}_{i} .
$$

It generates the canonical transformation

$$
\overline{\phi_{\mathfrak{d} \mathfrak{i a g} \mathfrak{g}}^{i}}: \quad \Theta_{i}=\frac{\tilde{p}_{i}}{\tilde{\beta}_{i}} \quad \vartheta_{i}-\pi=\tilde{\beta}_{i} \tilde{q}_{i}, \quad \mathrm{y}_{i}=\tilde{\mathrm{y}}_{i}, \quad \mathrm{x}_{i}=\tilde{\mathrm{x}}_{i}+\frac{\partial_{\mathrm{y}_{i}} \beta_{i}\left(\tilde{\mathrm{y}}_{i}\right)}{\beta_{i}\left(\tilde{\mathrm{y}}_{i}\right)} \tilde{p}_{i} \tilde{q}_{i}
$$

which transforms $\overline{\overline{f_{\mathcal{P}}^{i, i+1}}}$ into

$$
\overline{\overline{f_{\mathfrak{d} \mathfrak{i a g}, i}}}=\overline{\overline{f_{\mathcal{P}}^{i, i+1}}} \circ \overline{\phi_{\mathfrak{d} \mathfrak{i} \mathfrak{a g}}^{i}}=\tilde{\mathcal{A}}_{i}\left[\tilde{\mathrm{E}}_{i}+\tilde{\Omega}_{i} \frac{\tilde{p}_{i}^{2}+\tilde{q}_{i}}{2}+\tilde{\mathcal{R}}_{i}\right]
$$

with

$$
\begin{gathered}
\tilde{\beta}_{i}:=\beta\left(\tilde{\mathrm{y}}_{i}\right), \quad \tilde{\mathcal{A}}_{i}:=\mathcal{A}_{i}\left(\tilde{\mathrm{y}}_{i}\right), \quad \tilde{\mathrm{E}}_{i}:=C\left(\tilde{\mathrm{y}}_{i}\right), \quad \tilde{\Omega}_{i}:=\Omega\left(\tilde{\mathrm{y}}_{i}\right), \\
\tilde{\mathcal{R}}_{i}:=\mathcal{R}_{i} \circ \phi_{\mathfrak{d i a g}}^{i}=\tilde{\tau}_{1, i} \tilde{p}_{i}^{4}+\tilde{\tau}_{2, i} \tilde{p}_{i}^{2} \tilde{q}_{i}^{2}+\tilde{\tau}_{3, i} \tilde{q}_{i}^{4}+\mathrm{O}\left(\tilde{p}_{i}, \tilde{q}_{i}\right)^{6} \\
\tilde{\tau}_{1, i}:=\frac{\tau_{1}\left(\tilde{\mathrm{y}}_{i}\right)}{\tilde{\beta}_{i}^{4}}, \quad \tilde{\tau}_{2, i}:=\tau_{2}\left(\tilde{\mathrm{y}}_{i}\right), \quad \tilde{\tau}_{3, i}:=\tau_{3}\left(\tilde{\mathrm{y}}_{i}\right) \tilde{\beta}_{i}^{4}
\end{gathered}
$$

To compute the domain of $\overline{\phi_{\mathfrak{d} \mathfrak{i a g}}^{i}}$, we use the following inequalities, which readily follow from the definitions:

$$
\hat{\mathfrak{c}}_{i} \frac{\sqrt{\theta_{i}}}{\mathrm{G}_{n}^{+}} \leq\left|\beta_{i}\right| \leq \frac{1}{\hat{\mathfrak{c}}_{i}} \frac{\sqrt{\theta_{i}}}{\mathrm{G}_{n}^{-}}
$$

and

$$
\left|\frac{\partial_{\mathrm{y}_{i}} \beta_{i}\left(\tilde{\mathrm{y}}_{i}\right)}{\beta_{i}\left(\tilde{\mathrm{y}}_{i}\right)}\right| \leq \frac{1}{\hat{\mathfrak{c}}_{i} \theta_{i}} .
$$

We then see that, choosing a suitable $\tilde{\mathfrak{c}}_{i} \leq \hat{\mathfrak{c}}_{i}$, and the domain

$$
\widetilde{\mathfrak{B}_{i}}: \quad\left|\left(\tilde{p}_{i}, \tilde{q}_{i}\right)\right| \leq \tilde{\varepsilon}_{i}=\tilde{\mathfrak{c}}_{i} \sqrt{\theta_{i}} \quad \tilde{\mathrm{y}}_{i} \in \mathcal{A}_{\theta_{i}}^{i} \times \mathrm{X}_{\theta_{i}}^{i} \quad \tilde{\mathrm{x}}_{i} \in \mathbb{T}_{\frac{3}{4} s}^{m_{i}}
$$

inequalities 1 (5.100) are verified, as desired. Now we look for another canonical transformation

$$
\overline{\phi_{\mathfrak{b i r}}^{i}}: \quad\left(p_{i}^{*}, q_{i}^{*}, \mathrm{y}_{i}^{*}, \mathrm{x}_{i}^{*}\right) \rightarrow\left(\tilde{p}_{i}, \tilde{q}_{i}, \tilde{\mathrm{y}}_{i}, \tilde{\mathrm{x}}_{i}\right) \quad\left(\mathrm{y}_{i}^{*}=\tilde{\mathrm{y}}_{i}\right)
$$

${ }^{1}$ Compare 4.50). 
defined in a analogous domain

$$
{\overline{\mathfrak{B}_{i}}}^{*}:=\overline{\mathfrak{B}_{i}}: \quad\left|\left(p_{i}^{*}, q_{i}^{*}\right)\right| \leq \varepsilon_{i}=\mathfrak{c}_{i}^{*} \sqrt{\theta_{i}} \quad \mathrm{y}_{i}^{*} \in \mathcal{A}_{\theta_{i}}^{i} \times \mathrm{X}_{\theta_{i}}^{i} \quad \mathrm{x}_{i}^{*} \in \mathbb{T}_{\frac{s}{2}}^{m_{i}}
$$

with $\mathfrak{c}_{i}^{*}=: \mathfrak{c}_{i} \leq \tilde{\mathfrak{c}}_{i} / 2$, such that

$$
\overline{\overline{f_{\mathfrak{d i a g}, i}}} \circ \overline{\phi_{\mathfrak{b i r}}^{i}}=\overline{\mathrm{h}_{\mathfrak{s e c}}^{i}}
$$

satisfies the thesis of the lemma. We aim to apply Theorem D.1, with

$$
\mathrm{h}=\tilde{\mathrm{E}}_{i}+\tilde{\Omega}_{i} \frac{\tilde{p}_{i}^{2}+\tilde{q}_{i}}{2}, \quad f=\mathcal{R}_{i}, \quad \varepsilon=2 \mathfrak{c}_{i}^{*} \sqrt{\theta_{i}}, \quad \bar{\varepsilon}=\mathfrak{c}_{i}^{*} \sqrt{\theta_{i}} .
$$

We have to check that inequalities (D.152) are satisfied. We can take $\mathfrak{a}$ and $\mathfrak{e}$ as it follows from the following inequalities, which, in turn, are easily implied by the definitions

$$
\begin{aligned}
\inf _{\mathfrak{B}_{i}^{*}}|\partial \mathrm{h}| & =\inf _{\mathfrak{B}_{i}^{*}}\left|\tilde{\Omega}_{i}\right| \geq \frac{\check{\mathfrak{c}}_{i}\left|\mathrm{G}_{n}^{-}\right|^{2}}{\theta_{i}}=: \mathfrak{a} \\
\sup _{\mathfrak{B}_{i}^{*}}\left|\tilde{\mathcal{R}}_{i}\right| & \leq \sup _{\mathfrak{D}_{i}^{*}}\left|\mathcal{R}_{i}\right| \leq \frac{1}{\check{\mathfrak{c}}_{i}} \max \sup _{\mathcal{D}_{i}^{*}}\left\{\frac{\left(\Theta_{i}^{*}\right)^{4}}{\left(\mathrm{G}_{n}^{-}\right)^{2}},\left(\vartheta_{i}^{*}-\pi\right)^{2} \Theta_{i}^{2},\left(\mathrm{G}_{n}^{+}\right)^{2}\left(\vartheta_{i}^{*}-\pi\right)^{4}\right\} \\
& \leq \frac{\left(\mathfrak{c}^{*}\right)^{4}\left(\mathrm{G}_{n}^{+}\right)^{2}}{\overline{\mathfrak{c}}_{i}}=: \mathfrak{e}
\end{aligned}
$$

Here, we have used that, for $\left|\left(p_{i}^{*}, q_{i}^{*}\right)\right| \leq 2 \mathfrak{c}^{*} \sqrt{\theta_{i}},\left(\Theta_{i}^{*}, \vartheta_{i}^{*}\right):=\left(\overline{\phi_{\mathfrak{d} \mathfrak{i a g}}^{i}}\right)^{-1}\left(p_{i}^{*}, q_{i}^{*}\right)$ verifies

$$
\begin{aligned}
& \left|\Theta_{i}^{*}\right|=\frac{\left|p_{i}^{*}\right|}{\left|\beta_{i}\right|} \leq 2 \mathfrak{c}^{*} \sqrt{\theta_{i}} \frac{\mathrm{G}_{n}^{+}}{\hat{\mathfrak{c}}_{i} \sqrt{\theta_{i}}}=2 \frac{\mathfrak{c}^{*} \mathrm{G}_{n}^{+}}{\hat{\mathfrak{c}}_{i}} \\
& \left|\vartheta_{i}^{*}-\pi\right|=\left|q_{i}^{*}\right|\left|\beta_{i}\right| \leq \frac{2 \mathfrak{c}^{*} \sqrt{\theta_{i}}}{\mathfrak{c}_{1}} \frac{\sqrt{\theta_{i}}}{\mathrm{G}_{n}^{-}}=2 \frac{\mathfrak{c}^{*}}{\hat{\mathfrak{c}}_{i}} \frac{\theta_{i}}{\mathrm{G}_{n}^{-}}
\end{aligned}
$$

We then have that condition (D.152) holds, provided one takes

$$
\mathfrak{c}^{*}:=\min \left\{\frac{\mathrm{G}_{n}^{-}}{\mathrm{G}_{n}^{+}} \sqrt{\check{\mathfrak{c}}_{i} \overline{\mathfrak{c}}_{i}}, \frac{\tilde{\mathfrak{c}}_{i}}{2}\right\} .
$$

From (5.102), one easily computes that the fourth order term of $\overline{\mathrm{h}_{\mathfrak{s e c}}^{i}}$ corresponds to be as in (5.98), with

$$
\tau_{i}=\frac{3}{2} \tau_{1, i}^{*}+\frac{1}{2} \tau_{2, i}^{*}+\frac{3}{2} \tau_{3, i}^{*} \quad \tau_{j, i}^{*}:=\tilde{\tau}_{j, i}\left(\mathrm{y}_{i}^{*}\right) .
$$

Finally, properties (3.45) easily follow from the construction.

5.2.2. Construction of $\phi_{\mathfrak{i n t}}^{1}, \cdots, \phi_{\mathfrak{i n t}}^{n-2}(n \geq 3)$. We have to solve (5.80), assuming that Proposition 5.2 holds, up to rank $h-1$. Accordingly to (5.74), (5.75) and letting

$$
\overline{\Phi_{\mathfrak{i n t}}^{n-h+1}}:=\overline{\phi_{\mathfrak{i n t}}^{n-h+1}} \circ \ldots \circ \overline{\phi_{\mathfrak{i n t}}^{n-1}}
$$


we may split

$$
\begin{aligned}
f_{\mathfrak{n o r m}, h-1}^{n-h} & =\sum_{j=n-h+1}^{n} \Pi_{h-1}\left(\overline{f_{\mathcal{P}}^{n-h, j}}(\geq 2) \circ \overline{\Phi_{\mathfrak{i n t}}^{n-h+1}}\right)+\widetilde{f_{\mathfrak{s e c}, h-1}^{n-h}} \\
& =\Pi_{h-1}\left(\overline{f_{\mathcal{P}}^{n-h, n-h+1}}(2) \circ \overline{\Phi_{\mathfrak{i n t}}^{n-h+1}}\right)+\Pi_{h-1}\left(\overline{f_{\mathcal{P}}^{n-h, n-h+1}}(\geq 3) \circ \overline{\Phi_{\mathfrak{i n t}}^{n-h+1}}\right) \\
& +\sum_{j=n-h+2}^{n} \Pi_{h-1}\left({\overline{f_{\mathcal{P}}^{n-h, j}}}^{(\geq 2)} \circ \overline{\Phi_{\mathfrak{i n t}}^{n-h+1}}\right)+\widetilde{f_{\mathfrak{s e c}, h-1}^{n-h}} \\
& ={\overline{f_{\mathcal{P}}^{n-h, n-h+1}}}^{(2)}+\Pi_{h-1}\left({\overline{f_{\mathcal{P}}^{n-h, n-h+1}}}^{(2)} \circ \overline{\Phi_{\mathfrak{i n t}}^{n-h+1}}\right) \\
& +\Pi_{h-1}\left({\overline{f_{\mathcal{P}}^{n-h, n-h+1}}}^{(\geq 3)} \circ \overline{\Phi_{\mathfrak{i n t}}^{n-h+1}}\right) \\
& +\sum_{j=n-h+2}^{n} \Pi_{h-1}\left({\overline{f_{\mathcal{P}}^{n-h, j}}}^{(\geq 2)} \circ \overline{\Phi_{\mathfrak{i n t}}^{n-h+1}}\right)+\widetilde{f_{\mathfrak{s e c}, h-1}^{n-h}}
\end{aligned}
$$

where

$$
\begin{aligned}
{\overline{f_{\mathcal{P}}^{n-h, n-h+1}}}^{(\geq 3)}:={\overline{f_{\mathcal{P}}^{n-h, n-h+1}}}^{(\geq 2)}-{\overline{f_{\mathcal{P}}^{n-h, n-h+1}}}^{(2)}{ }^{(2)} & {\overline{f_{\mathcal{P}}^{n-h, n-h+h+1}}}^{(2)}-{\overline{\overline{f_{\mathcal{P}}^{n-h, n-h+1}}}}^{(2)}
\end{aligned}
$$

and ${\overline{\overline{f_{\mathcal{P}}^{n-h, n-h+1}}}}^{(2)}$ as in Lemma 3.4. Note that we have used that ${\overline{\overline{f_{\mathcal{P}}^{n-h, n-h+1}}}}^{(2)}$ is

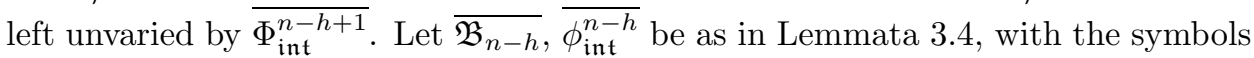
$\left(\Theta_{n-h}, \vartheta_{n-h}\right), \mathrm{y}_{n-h}, \mathrm{x}_{n-h}$ of that lemma corresponding to

$$
\begin{aligned}
\overline{\mathrm{t}}_{n-h}^{(h-1)} & :=\left(\Theta_{n-h}^{(h-1)}, \vartheta_{n-h}^{(h-1)}\right) \\
\overline{\mathrm{y}}_{n-h}^{(h-1)} & :=\left(\chi_{n-h-1}^{(h-1)}, \chi_{n-h}^{(h-1)}, \chi_{n-h+1}^{(h-1)}, \Lambda_{n-h}^{(h-1)}, \Lambda_{n-h+1}^{(h-1)}\right) \\
\overline{\mathrm{x}}_{n-h}^{(h-1)} & :=\left(\kappa_{n-h-1}^{(h-1)}, \kappa_{n-h}^{(h-1)}, \kappa_{n-h+1}^{(h-1)}, \ell_{n-h}^{(h-1)}, \ell_{n-h+1}^{(h-1)}\right)
\end{aligned}
$$

and the symbols $\left(p_{n-h}, q_{n-h}\right), \mathrm{y}_{n-h}^{*}, \mathrm{x}_{n-h}^{*}$ to

$$
\begin{aligned}
& \overline{\mathrm{z}}_{n-h}^{*(h)}:=\left(p_{n-h}^{*(h)}, q_{n-h}^{*(h)}\right) \\
& \overline{\mathrm{y}}_{n-h}^{*(h)}:=\left(\begin{array}{lll}
\chi_{n-h-1}^{*(h)}, & \chi_{n-h}^{*(h)}, \chi_{n-h+1}^{*(h)}, \Lambda_{n-h}^{*(h)}, \Lambda_{n-h+1}^{*(h)}
\end{array}\right) \\
& \overline{\mathrm{x}}_{n-h}^{*(h)}:=\left(\kappa_{n-h-1}^{*(h)}, \kappa_{n-h}^{*(h)}, \kappa_{n-h+1}^{*(h)}, \ell_{n-h}^{*(h)}, \ell_{n-h+1}^{*(h)}\right) .
\end{aligned}
$$

Defining

$$
\begin{aligned}
& \mathrm{t}^{*(h)}:=\left(\Theta^{*(h)}, \vartheta^{*(h)}\right) \\
& \mathrm{z}^{*(h)}:=\left(p^{*(h)}, q^{*(h)}\right) \\
& \mathrm{y}^{*(h)}:=\left(\chi^{*(h)}, \Lambda^{*(h)}\right) \\
& \mathrm{x}^{*(h)}:=\left(\kappa^{*(h)}, \ell^{*(h)}\right)
\end{aligned}
$$

in an alagous way as in (5.70), we regard $\overline{\phi_{\mathfrak{i n t}}^{n-h}}$ as a map on the set

$$
\overline{\mathfrak{D}_{\mathfrak{i n t}}^{h}}:=\left\{\left(\mathrm{t}^{*(h)}, \mathrm{z}^{*(h)}, \mathrm{y}^{*(h)}, \mathrm{x}^{*(h)}\right):\left(\overline{\mathrm{z}}_{n-h}^{*(h)}, \overline{\mathrm{y}}_{n-h}^{*(h)}, \overline{\mathrm{x}}_{n-h}^{*(h)}\right) \in \overline{\mathfrak{B}_{n-h}}\right\}
$$


extended via the identity on the extra-coordinates. We then have that $\overline{\phi_{\mathfrak{i n t}}^{n-h}}$ transforms $f_{\mathfrak{s e c}, h-1}^{n-h}$ into

$$
\overline{f_{\mathfrak{s e c}, h-1}^{n-h}}:=f_{\mathfrak{s e c}, h-1}^{n-h} \circ \overline{\phi_{\mathfrak{i n t}}^{n-h}}=\overline{\mathrm{h}_{\mathfrak{s e c}}^{n-h}}+\overline{\mathrm{f}_{\mathfrak{s e c}}^{n-h}}
$$

where

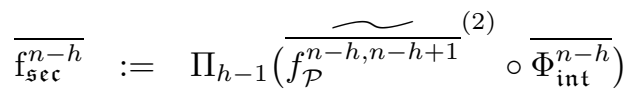

$$
\begin{aligned}
& +\Pi_{h-1}\left(\overline{f_{\mathcal{P}}^{n-h, n-h+1}}(\geq 3) \circ \overline{\Phi_{\mathfrak{i n t}}^{n-h}}\right)+\sum_{j=n-h+2}^{n} \Pi_{h-1}\left(\overline{f_{\mathcal{P}}^{n-h, j}}(\geq 2) \circ \overline{\Phi_{\mathfrak{i n t}}^{n-h}}\right) \\
& +\widetilde{f_{\mathfrak{s e c}, h-1}^{n-h}} \odot \overline{\phi_{\mathfrak{i n t}}^{n-h}} .
\end{aligned}
$$

Here, we have used $\overline{\Phi_{\mathfrak{i n t}}^{n-h}}=\overline{\Phi_{\mathfrak{i n t}}^{n-h+1}} \circ \overline{\phi_{\mathfrak{i n t}}^{n-h}}$; that $\Pi_{h-1}$ and $\overline{\phi_{\mathfrak{i n t}}^{n-h}}$ commute and observe that $\overline{\mathfrak{D}_{\mathfrak{i n t}}^{h}}$ has the form of $\mathfrak{D}_{\mathfrak{i n t}}^{h}$ in (5.79), with $\hat{\mathfrak{c}}_{h}$ replaced by a suitable $\hat{\mathfrak{c}}_{h}^{\prime}$ of the same form. The function $\overline{f_{\mathfrak{s e c}}^{n-h}}$ satisfies the following two properties:

- It depends only on

$$
\left(p_{n-h}^{*(h)}, q_{n-h}^{*(h)}, \tilde{\mathrm{y}}^{*(h)}\right)
$$

where $\tilde{y}^{*(h)}$ is defined analogously to (5.83);

- is uniformly bounded by the right hand side of the first inequality in (5.76) (this follows from the definition in (5.103) );

- is even for

$$
\left(p_{n-h}^{*(h)}, q_{n-h}^{*(h)}\right) \rightarrow-\left(p_{n-h}^{*(h)}, q_{n-h}^{*(h)}\right) .
$$

Proceeding in a similar way as we did for the construction of $\overline{\phi_{\mathfrak{b i r}}^{i}}$ in the proof of Lemma 5.2, we may apply Theorem D.1, with

$$
\begin{aligned}
& \mathrm{h}=\overline{\mathrm{h}_{\mathfrak{s e c}}^{n-h}}, \quad f=\overline{\mathrm{f}_{\mathfrak{s e c}}^{n-h}}, \quad(P, Q)=\left(p_{n-h}^{*(h)}, q_{n-h}^{*(h)}\right) \\
& \left(P^{\prime}, Q^{\prime}\right)=\hat{\mathrm{z}}_{n-h}^{*(h)}, \quad \mathrm{y}=\mathrm{y}_{n-h}^{*(h)}, \quad \mathrm{x}=\mathrm{x}_{n-h}^{*(h)} .
\end{aligned}
$$

with $\mathrm{y}_{n-h}^{*(h)}, \mathrm{x}_{n-h}^{*(h)}$ defined analogously to $\mathrm{y}_{*, n-h}^{(h)}, \mathrm{x}_{*, n-h}^{(h)}$ in (5.81) and $\hat{\mathrm{z}}_{n-h}^{(h)}$ defined analogously to $\hat{\mathrm{z}}_{*, n-h}^{(h)}$ in (5.83). We then find another domain $\mathfrak{D}_{\mathfrak{i n t}}^{h}$ as in (5.79) and another real-analytic transformation

$$
\phi_{*, \mathfrak{i n} \mathfrak{t}}^{n-h}: \quad\left(\mathrm{t}_{*}^{(h)}, \mathrm{z}_{*}^{(h)}, \mathrm{y}_{*}^{(h)}, \mathrm{x}_{*}^{(h)}\right) \in \mathfrak{D}_{\mathfrak{i n t}}^{h} \rightarrow\left(\mathrm{t}^{*(h)}, \mathrm{z}^{*(h)}, \mathrm{y}^{*(h)}, \mathrm{x}^{*(h)}\right) \in \overline{\mathfrak{D}_{\mathfrak{i n t}, h}}
$$

such that

$$
\widetilde{f_{\mathfrak{s e c}, h-1}^{n-h}}:=\overline{f_{\mathfrak{s e c}, h-1}^{n-h}} \circ \phi_{*, \mathfrak{i n t}}^{n-h}=f_{\mathfrak{s e c}, h-1}^{n-h} \circ \overline{\phi_{\mathfrak{i n t}}^{n-h}} \circ \phi_{*, \mathfrak{i n t}}^{n-h}=\mathrm{h}_{\mathfrak{s e c}}^{n-h}
$$

as desired, depends only on $\hat{\mathrm{y}}_{*, n-h}^{(h)}$ in (5.71), and hence (5.80) is satisfied. That $\phi_{*, \mathfrak{i n t}}^{n-h}$ may be also chosen of a form analogous to (5.82) , with $\Theta_{n-h}^{(h-1)}, \vartheta_{n-h}^{(h-1)}, \hat{\mathrm{z}}^{(h-1)}$, $\mathrm{y}_{n-h}^{(h-1)}, \mathrm{x}_{n-h}^{(h-1)}$ replaced by $p^{*(h)}, q^{*(h)}, \hat{\mathrm{z}}^{*(h)}, \mathrm{y}^{*(h)}, \mathrm{x}^{*(h)}$ also easily follows from the properties bove. Therefore the composition

$$
\phi_{\mathfrak{i n t}}^{n-h}:=\overline{\phi_{\mathfrak{i n t}}^{n-h}} \circ \phi_{*, \mathfrak{i n t}}^{n-h}
$$

has again the form in (5.82) and satisfies (5.80), as wanted. 
5.2.3. Construction of $\phi_{\text {norm }}^{1}, \cdots, \phi_{\text {norm }}^{n-2}(n \geq 3)$. In this section we aim to determine, for $n \geq 3$ and $1 \leq h \leq n-2$, a transformation $\phi_{\mathfrak{n o r m}}^{n-h}$ solving (5.86)(5.87), assuming the Proposition 5.2 holds up to rank $(h-1)$ and that $\phi_{\mathfrak{i n t}}^{n-h}$ has been constructed.

We switch from the coordinates $\left(\chi_{*}^{(h)}, \kappa_{*}^{(h)}\right)$ defined implicitly via the right hand side of (5.86) to the auxiliary coordinates

$$
\mathrm{G}_{\mathfrak{a u x}}^{(h)}=\left(\mathrm{G}_{\mathfrak{a u x}, 1}^{(h)}, \cdots, \mathrm{G}_{\mathfrak{a} \mathfrak{u} \mathfrak{x}, n}^{(h)}\right), \quad \mathrm{g}_{\mathfrak{a u x}}^{(h)}=\left(\mathrm{g}_{\mathfrak{a u x}, 1}^{(h)}, \cdots, \mathrm{g}_{\mathfrak{a} \mathfrak{u} \mathfrak{x}, n}^{(h)}\right)
$$

defined via the linear transformation

$$
\phi_{\mathfrak{a u x}}^{n-h}:\left\{\begin{array}{l}
\chi_{*, i-1}^{(h)}=\mathrm{G}_{\mathfrak{a u x}, i}^{(h)}+\cdots+\mathrm{G}_{\mathfrak{a u x}, n}^{(h)} \\
\kappa_{*, i-1}^{(h)}=\mathrm{g}_{\mathfrak{a u x}, i}^{(h)}-\mathrm{g}_{\mathfrak{a u x}, i-1}^{(h)}
\end{array}\right.
$$

with $1 \leq i \leq n$ and $\mathrm{g}_{\mathfrak{a u x}, 0}:=0$. We regard $\phi_{\mathfrak{a u x}}^{n-h}$ as a transformation on all the coordinates, extending it as the identity on the remaining ones. We denote the new coordinates as

$$
\begin{aligned}
& \mathrm{t}_{\mathfrak{a} \mathfrak{u} \mathfrak{x}}^{(h)}:=\left\{\begin{array}{c}
\left(\Theta_{\mathfrak{a u x}, 1}^{(h)}, \cdots, \Theta_{\mathfrak{a u x}, n-h-1}^{(h)}, \vartheta_{\mathfrak{a u x}, 1}^{(h)}, \cdots, \vartheta_{\mathfrak{a} \mathfrak{u x}, n-h-1}^{(h)}\right) \\
\emptyset \geq 4,2 \leq h \leq n-2 \\
\emptyset \quad \text { otherwise }
\end{array}\right. \\
& \mathrm{z}_{\mathfrak{a u x}}^{(h)}:=\left(p_{\mathfrak{a u x}, n-h}^{(h)}, \cdots, p_{\mathfrak{a u x}, n-1}^{(h)}, q_{\mathfrak{a u x}, n-h}^{(h)}, \cdots, q_{\mathfrak{a u x}, n-1}^{(h)}\right) \\
& \mathrm{y}_{\mathfrak{a u x}}^{(h)}:=\left(\mathrm{G}_{\mathfrak{a u x}, 1}^{(h)}, \cdots, \mathrm{G}_{\mathfrak{a u x}, n}^{(h)}, \Lambda_{\mathfrak{a u x}, 1}^{(h)}, \cdots, \Lambda_{\mathfrak{a u x}, n}^{(h)}\right) \\
& \mathbf{x}_{\mathfrak{a u x}}^{(h)}:=\left(\mathrm{g}_{\mathfrak{a u x}, 1}^{(h)}, \cdots, \mathrm{g}_{\mathfrak{a u x}, n}^{(h)}, \ell_{\mathfrak{a u x}, 1}^{(h)}, \cdots, \ell_{\mathfrak{a u x}, n}^{(h)}\right)
\end{aligned}
$$

the new Hamiltonian as

$$
f_{\mathfrak{s e c}, \mathfrak{i n t}, \mathfrak{a u x}, h-1}\left(\mathrm{t}_{\mathfrak{a u x}}^{(h)}, \mathrm{z}_{\mathfrak{a u x}}^{(h)}, \mathrm{y}_{\mathfrak{a} \mathfrak{u x}}^{(h)}, \mathrm{x}_{\mathfrak{a u x}}^{(h)}\right):=f_{\mathfrak{s e c}, \mathfrak{i n t}, h-1} \circ \phi_{\mathfrak{a u x}}^{n-h}\left(\mathrm{t}_{\mathfrak{a u x}}^{(h)}, \mathrm{z}_{\mathfrak{a u x}}^{(h)}, \mathrm{y}_{\mathfrak{a} \mathfrak{u} \mathfrak{x}}^{(h)}, \mathrm{x}_{\mathfrak{a u x}}^{(h)}\right)
$$

Now we define the domain where we want to consider $f_{\mathfrak{s e c}, \text { int }, \mathfrak{a u x}, h-1}$. Firstly, we let

$$
\mathfrak{D}_{\mathfrak{i n t}, \mathfrak{a u x}}^{h}:=\left\{\left(\mathrm{t}_{\mathfrak{a u x}}^{(h)}, \mathrm{z}_{\mathfrak{a u x}}^{(h)}, \mathrm{y}_{\mathfrak{a} \mathfrak{x}}^{(h)}, \mathrm{x}_{\mathfrak{a u x}}^{(h)}\right): \quad\left(\mathrm{t}_{*}^{(h)}, \mathrm{z}_{*}^{(h)}, \mathrm{y}_{*}^{(h)}, \mathrm{x}_{*}^{(h)}\right) \in \mathfrak{D}_{\mathfrak{i n t}}^{h}\right\}
$$

where $\mathfrak{D}_{\mathfrak{i n t}}^{h}$ is defined in (5.79). Then $\mathfrak{D}_{\mathfrak{i n t}, \mathfrak{a u x}}^{h}$ is given by

$$
\mathfrak{D}_{\mathfrak{i n t}, \mathfrak{a u x}}^{h}=\mathrm{T}_{\hat{\mathfrak{c}}_{h} \theta}^{h} \times B_{\hat{\mathfrak{c}}_{h} \varepsilon_{n-h}}^{2} \times B_{\hat{\mathfrak{c}}_{h} \varepsilon}^{*, h-1} \times\left(\mathrm{G}_{*}\right)_{\overline{\mathfrak{c}}_{h} \theta, \overline{\mathfrak{c}}_{h} \bar{r}} \times \mathrm{A}_{\overline{\mathfrak{c}}_{h} \widetilde{r}^{(h)}} \times \mathbb{T}_{\overline{\mathfrak{c}}_{h} s}^{n} \times \mathbb{T}_{\overline{\mathfrak{c}}_{h} s}^{n}
$$

with

$$
\begin{aligned}
\left(\mathrm{G}_{*}\right)_{\overline{\mathfrak{c}}_{h} \theta, \overline{\mathfrak{c}} h \bar{r}}:= & \left(\mathrm{G}_{1}\right)_{\hat{\mathfrak{c}}_{1} \theta_{1}} \times \cdots \times\left(\mathrm{G}_{n-h}\right)_{\hat{\mathfrak{c}}_{n-h} \theta_{n-h}} \\
& \times\left(\mathrm{G}_{n-h+1}^{*}\right)_{\hat{\mathfrak{c}}_{n-h+1} \bar{r}_{n-h+1}} \times \cdots \times\left(\mathrm{G}_{n-1}^{*}\right)_{\hat{\mathfrak{c}}_{n-1} \bar{r}_{n-1}} .
\end{aligned}
$$


Next, for $1 \leq h^{\prime} \leq h$ and any fixed $\bar{\gamma}, \bar{K}>0$ and $\bar{\tau}>2$, we define

$$
\begin{aligned}
& \omega_{\mathfrak{s e c}}^{n-h^{\prime}}\left(\hat{y}_{\mathfrak{a u x}, n-h^{\prime}}^{(h)}\right):=
\end{aligned}
$$

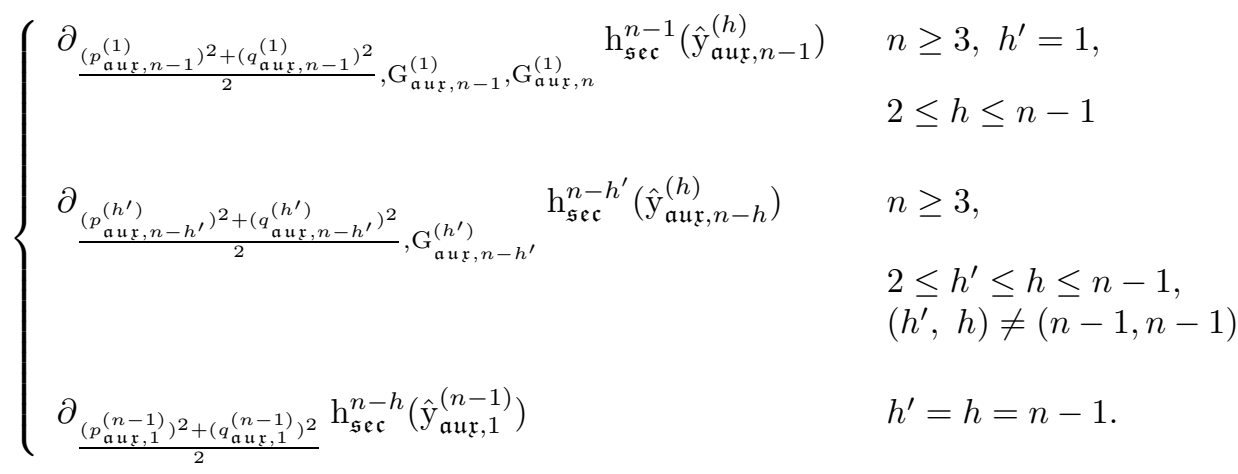

We then choose the following sub-domain of $\mathfrak{D}_{\mathfrak{i n t}, \mathfrak{a} \mathfrak{a x}}^{h}$

$$
\begin{aligned}
\mathfrak{D}_{\mathfrak{s e c}, \mathfrak{a u x}}^{h}:= & \left\{\left(\mathrm{t}_{\mathfrak{n o r m}, \mathfrak{a u x}}^{(h)}, \mathrm{z}_{\mathfrak{n o r m}, \mathfrak{a u x}}^{(h)}, \mathrm{y}_{\mathfrak{n o r m}, \mathfrak{a u x}}^{(h)}, \mathrm{x}_{\mathfrak{n o r m}, \mathfrak{a u x}}^{(h)}\right) \in \mathfrak{D}_{\mathfrak{i n t}, \mathfrak{a u x}}^{h}:\right. \\
& \left|\omega_{\mathfrak{s e c}}^{n-h^{\prime}} \cdot \mathrm{k}\right| \geq \frac{\left(a_{n-h^{\prime}}^{+}\right)^{2}}{\left(a_{n-h^{\prime}+1}^{-}\right)^{3} \theta_{n-h}} \frac{\bar{\gamma}}{\bar{K}^{\bar{\tau}}}, \\
& \left.\forall \mathrm{k} \in \mathbb{Z}^{j} \backslash\{0\},|\mathrm{k}|_{1} \leq \bar{K}, \quad \forall 2 \leq h^{\prime} \leq h\right\}
\end{aligned}
$$

Here $j$ is chosen to be 3,2 or 1 accordingly to the three cases above. The set $\mathfrak{D}_{\mathfrak{i n t}, \mathfrak{a u x}}^{h}$ is non-empty, if $\bar{\gamma}$ is chosen suitably small. Indeed, if we put

$$
\hat{\mathrm{y}}_{\mathfrak{a} \mathfrak{u x}, n-h}^{(h)}:=\left(\frac{\left(p_{\mathfrak{a u x}, n-h}^{(h)}\right)^{2}+\left(q_{\mathfrak{a u x}, n-h}^{(h)}\right)^{2}}{2}, \mathrm{G}_{\mathfrak{a} \mathfrak{u x}, n-h}^{(h)}, \Lambda_{\mathfrak{a u x}, n-h}^{(h)}, \hat{\mathrm{y}}_{\mathfrak{a u x}, n-h+1}^{(h)}\right)
$$

then standard quantitative arguments show that, for any fixed value

$$
\left(\bar{\Lambda}_{\mathfrak{a} \mathfrak{u} \mathfrak{x}, n-h}^{(h)}, \hat{\overline{\mathrm{y}}}_{\mathfrak{a} \mathfrak{u} \mathfrak{x}, n-h+1}^{(h)}\right) \in \Pi_{\Lambda_{\mathfrak{a} \mathfrak{u} \mathfrak{x}, n-h}^{(h)}, \hat{\mathrm{y}}_{\mathfrak{a} \mathfrak{u} \mathfrak{x}, n-h+1}^{(h)}} \mathfrak{D}_{\mathfrak{i n t}, \mathfrak{a u x}}^{h}
$$

the measure of the set $\mathcal{N}_{n-h} \subset B_{\overline{\mathfrak{c}}_{h} \varepsilon_{n-h}}^{2} \times \mathrm{G}_{n-h}$ of $\left(p_{\mathfrak{a u x}, n-h}^{(h)}, q_{\mathfrak{a u x}, n-h}^{(h)}, \mathrm{G}_{\mathfrak{a u x}, n-h}^{(h)}\right)$ where the inequality in (5.107) does not hold may be bounded as

$$
\operatorname{meas} \mathcal{N}_{n-h} \leq \frac{\bar{\gamma}}{\mathfrak{c}} \operatorname{meas}\left(B_{\overline{\mathfrak{c}}_{h} \varepsilon_{n-h}}^{2} \times \mathrm{G}_{n-h}\right)
$$

(where $\mathfrak{c}$ depends only on the semi-axes ratio and the masses), hence (5.68) follows. This is because $\omega_{\mathfrak{s e c}}^{n-h^{\prime}}\left(\hat{y}_{\mathfrak{a u x}, n-h}^{(h)}\right)$ is a diffeomorphism (Compare Appendix C). 
Now we inspect the form of $f_{\mathfrak{s e c}, \mathfrak{i n t}, \mathfrak{a u x}, h-1}$ in (5.105). Introducing the following symbols

$$
\begin{aligned}
& \mathrm{t}_{\mathfrak{a u x}, i}^{(h)}:=\left\{\begin{array}{c}
\left(\Theta_{\mathfrak{a u x}, i}^{(h)}, \cdots, \Theta_{\mathfrak{a u x}, n-h-1}^{(h)}, \vartheta_{\mathfrak{a u x}, i}^{(h)}, \cdots, \vartheta_{\mathfrak{a u x}, n-h-1}^{(h)}\right) \\
n \geq 4,2 \leq h \leq n-2,1 \leq i \leq n-h-1 \\
\emptyset \quad \text { otherwise }
\end{array}\right. \\
& \mathrm{y}_{\mathfrak{a u x}, i}^{(h)}:=\left(\mathrm{G}_{\mathfrak{a u x}, i}^{(h)}, \cdots, \mathrm{G}_{\mathfrak{a u x}, n}^{(h)}, \Lambda_{\mathfrak{a u x}, 1}^{(h)}, \cdots, \Lambda_{\mathfrak{a u x}, n}^{(h)}\right) \\
& \mathrm{x}_{\mathfrak{a u x}, i}^{(h)}:=\left(\mathrm{g}_{\mathfrak{a u x}, i}^{(h)}, \cdots, \mathrm{g}_{\mathfrak{a u x}, n}^{(h)}, \ell_{\mathfrak{a u x}, 1}^{(h)}, \cdots, \ell_{\mathfrak{a u x}, n}^{(h)}\right) \\
& \hat{\mathrm{y}}_{\mathfrak{a} \mathfrak{u x}, i}^{(h)}:=\left(\frac{\left(p_{\mathfrak{a u x}, i}^{(h)}\right)^{2}+\left(q_{\mathfrak{a u x}, i}^{(h)}\right)^{2}}{2}, \cdots, \frac{\left(p_{\mathfrak{a u x}, n-1}^{(h)}\right)^{2}+\left(q_{\mathfrak{a u x}, n-1}^{(h)}\right)^{2}}{2}, \mathrm{G}_{\mathfrak{a} \mathfrak{u x}, i}^{(h)}, \cdots,\right. \\
& \left.\mathrm{G}_{\mathfrak{a u x}, n}^{(h)}, \Lambda_{\mathfrak{a u x}, i}^{(h)}, \cdots, \Lambda_{\mathfrak{a u x}, n}^{(h)}\right) \\
& \hat{\mathbf{x}}_{\mathfrak{a u x}, i}^{(h)}:=\left\{\begin{array}{c}
\left(\mathrm{g}_{\mathfrak{a u x}, i+1}^{(h)}-\mathrm{g}_{\mathfrak{a u x}, i}^{(h)}, \begin{array}{c}
\left.\cdots, \mathrm{g}_{\mathfrak{a u x}, n-h}^{(h)}-\mathrm{g}_{\mathfrak{a u x}, n-h-1}^{(h)}\right) \\
n \geq 4 \& 1 \leq h-1 \leq n-3
\end{array}\right. \\
\emptyset \quad \text { otherwise }
\end{array}\right. \\
& \hat{\mathrm{X}}_{\mathfrak{a u x}, i}^{(h)}:=\left\{\begin{array}{l}
\left(\mathrm{G}_{\mathfrak{a u x}, i}^{(h)}, \cdots, \mathrm{G}_{\mathfrak{a u x}, n-h}^{(h)}\right) \quad n \geq 4 \& 1 \leq h-1 \leq n-3 \\
\emptyset \quad \text { otherwise }
\end{array}\right. \\
& \mathrm{z}_{\mathfrak{a u x}, n-h}^{(h)}:=\left(p_{\mathfrak{a u x}, n-h}^{(h)}, q_{\mathfrak{a u x}, n-h}^{(h)}\right), \quad \hat{\mathrm{z}}_{\mathfrak{n o r m}, j}^{(h)}:=p_{\mathfrak{n o r m}, j}^{(h)}+\mathrm{i} q_{\mathfrak{n o r m}, j}^{(h)} \\
& \tilde{\mathrm{y}}_{\mathfrak{a} \mathfrak{u} x, i}^{(h)}:=\left(\frac{\left(p_{\mathfrak{a u x}, n-h+1}^{(h)}\right)^{2}+\left(q_{\mathfrak{a u x}, n-h+1}^{(h)}\right)^{2}}{2}, \cdots, \frac{\left(p_{\mathfrak{a u x}, n-1}^{(h)}\right)^{2}+\left(q_{\mathfrak{a u x}, n-1}^{(h)}\right)^{2}}{2},\right. \\
& \left.\mathrm{G}_{\mathfrak{a u x}, i}^{(h)}, \cdots, \mathrm{G}_{\mathfrak{a u x}, n}^{(h)}, \Lambda_{\mathfrak{a u x}, i}^{(h)}, \cdots, \Lambda_{\mathfrak{a u x}, n}^{(h)}\right) \\
& \tilde{\mathrm{y}}_{\mathfrak{a} \mathfrak{u x}}^{(h)}:=\tilde{\mathrm{y}}_{\mathfrak{a u x}, 1}^{(h)}, \quad \hat{\mathbf{x}}_{\mathfrak{a u x}}^{(h)}:=\hat{\mathrm{x}}_{\mathfrak{a u x}, 1}^{(h)}, \quad \hat{\mathrm{X}}_{\mathfrak{a u x}}^{(h)}:=\hat{\mathrm{X}}_{\mathfrak{a u x}, 1}^{(h)},
\end{aligned}
$$

by means of (5.85), we have

$$
\begin{aligned}
& f_{\mathfrak{s e c}, \text { int }, \mathfrak{a u x}, h-1}\left(\mathrm{t}_{\mathfrak{a u x}}^{(h)}, \mathrm{z}_{\mathfrak{a u x}}^{(h)}, \mathrm{y}_{\mathfrak{a u x}}^{(h)}, \mathrm{x}_{\mathfrak{a u x}}^{(h)}\right) \\
& \quad=\mathrm{h}_{\mathfrak{s e c}, h}\left(\hat{\mathrm{y}}^{(h)}\right)+f_{\mathfrak{n o r m}, \mathfrak{i n t}, \mathfrak{a u x}, h-1}\left(\mathrm{t}_{\mathfrak{a u x}}^{(h)}, \mathrm{z}_{\mathfrak{a u x}, n-h}^{(h)}, \tilde{\mathrm{y}}_{\mathfrak{a u x}}^{(h)}, \hat{\mathrm{x}}_{\mathfrak{a u x}}^{(h)}\right) \\
& \quad=\sum_{i=n-h}^{n-1} \mathrm{~h}_{\mathfrak{s e c}}^{i}\left(\hat{\mathrm{y}}_{i}^{(h)}\right) \\
& \quad+\sum_{i=1}^{n-h-1} f_{\mathfrak{n o r m}, \mathfrak{i n t}, \mathfrak{a u x}, h-1}^{i}\left(\mathrm{t}_{\mathfrak{a u x}, i}^{(h)}, \mathrm{z}_{\mathfrak{a u x}, n-h}^{(h)}, \tilde{\mathrm{y}}_{\mathfrak{a u x}, i}^{(h)}, \hat{\mathrm{x}}_{\mathfrak{a u x}, i}^{(h)}\right)
\end{aligned}
$$

where we have let

$f_{\mathfrak{n o r m}, \mathfrak{i n t}, \mathfrak{a u x}, h-1}:=f_{\mathfrak{n o r m}, \mathfrak{i n t}, h-1} \circ \phi_{\mathfrak{a u x}}^{n-h}, \quad f_{\mathfrak{n o r m}, \mathfrak{i n t}, \mathfrak{a u x}, h-1}^{i}:=f_{\mathfrak{n o r m}, \mathfrak{i n t}, h-1}^{i} \circ \phi_{\mathfrak{a u x}}^{n-h}$.

On the domain $\mathfrak{D}_{\mathfrak{s e c}, \mathfrak{a u x}}^{h}$ specified in (5.107), we aim to construct and real-analytic and canonical transformation

$$
\begin{aligned}
& \phi_{\mathfrak{n o r m}, \mathfrak{a u x}}^{n-h}:\left(\mathrm{t}_{\mathfrak{n o r m}, \mathfrak{a u x}}^{(h)}, \mathrm{z}_{\mathfrak{n o r m}, \mathfrak{a u x}}^{(h)}, \mathrm{y}_{\mathfrak{n o r m}, \mathfrak{a u x}}^{(h)}, \mathrm{x}_{\mathfrak{n o r m}, \mathfrak{a u x}}^{(h)}\right) \\
& \quad \in \mathfrak{D}_{\mathfrak{s e c}, \mathfrak{a u x}}^{h} \rightarrow\left(\mathrm{t}_{\mathfrak{a u x}}^{(h)}, \mathrm{z}_{\mathfrak{a u x}}^{(h)} \mathrm{y}_{\mathfrak{a u x}}^{(h)}, \mathrm{x}_{\mathfrak{a u x}}^{(h)}\right) \in \mathfrak{D}_{\mathfrak{i n t}, \mathfrak{a u x}}^{h}
\end{aligned}
$$


such that the transformed Hamiltonian

$$
f_{\mathfrak{s e c}, \mathfrak{a u x}, h}:=f_{\mathfrak{s e c}, \mathfrak{i n t}, \mathfrak{a u x}, h-1} \circ \phi_{\mathfrak{n o r m}, \mathfrak{a u x}}^{n-h}
$$

has the form

$$
\begin{aligned}
f_{\mathfrak{s e c}, \mathfrak{a u x}, h} & =\mathrm{h}_{\mathfrak{s e c}, h}\left(\hat{\mathrm{y}}_{\mathfrak{n o r m}, \mathfrak{a u x}}^{(h)}\right)+f_{\mathfrak{n o r m}, \mathfrak{a u x}, h}\left(\mathrm{t}_{\mathfrak{n o r m}, \mathfrak{a u x}}^{(h)}, \hat{\mathrm{y}}_{\mathfrak{n o r m}, \mathfrak{a u x}}^{(h)}, \hat{\mathrm{x}}_{\mathfrak{n o r m}, \mathfrak{a u x}}^{(h)}\right) \\
& =\sum_{i=n-h}^{n-1} \mathrm{~h}_{\mathfrak{s e c}}^{i}\left(\hat{\mathrm{y}}_{\mathfrak{n o r m}, \mathfrak{a u x}, i}^{(h)}\right) \\
& +\sum_{i=1}^{n-h-1} f_{\mathfrak{n o r m}, \mathfrak{a u x}, h}^{i}\left(\mathrm{t}_{\mathfrak{n o r m}, \mathfrak{a u x}, i}^{(h)}, \hat{\mathrm{y}}_{\mathfrak{n o r m}, \mathfrak{a u x}, i}^{(h)}, \hat{\mathrm{x}}_{\mathfrak{n o r m}, \mathfrak{a u x}, i}^{(h)}\right) \\
& +f_{\mathfrak{e x p}, \mathfrak{s e c}, \mathfrak{a u x}, h}\left(\mathrm{t}_{\mathfrak{n o r m}, \mathfrak{a u x}}^{(h)}, \mathrm{z}_{\mathfrak{n o r m}, \mathfrak{a u x}}^{(h)}, \mathrm{y}_{\mathfrak{n o r m}, \mathfrak{a u x}}^{(h)}, \mathrm{x}_{\mathfrak{n o r m}, \mathfrak{a u x}}^{(h)}\right)
\end{aligned}
$$

where

$$
\begin{aligned}
& \hat{\mathbf{x}}_{\mathfrak{n o r m}, \mathfrak{a u x}, i}^{(h)}:=\left\{\begin{array}{l}
\left(\mathrm{g}_{\mathfrak{n o r m}, \mathfrak{a u x}, i+1}^{(h)}-\mathrm{g}_{\mathfrak{n o r m}, \mathfrak{a u x}, i}^{(h)}, \cdots, \mathrm{g}_{\mathfrak{n o r m}, \mathfrak{a u x}, n-h-1}^{(h)}\right. \\
\left.-\mathrm{g}_{\mathfrak{n o r m}, \mathfrak{a u x}, n-h-2}^{(h)}\right) \\
\emptyset \quad \text { otherwise }
\end{array}\right. \\
& \hat{\mathbf{y}}_{\mathfrak{n o r m}, \mathfrak{a u x}, i}^{(h)}:=\left(\frac{\left(p_{\mathfrak{n o r m}, \mathfrak{a u x}, n-h}^{(h)}\right)^{2}+\left(q_{\mathfrak{n o r m}, \mathfrak{a u x}, n-h}^{(h)}\right)^{2}}{2}, \cdots,\right. \\
& \frac{\left(p_{\mathfrak{n o r m}, \mathfrak{a u x}, n-1}^{(h)}\right)^{2}+\left(q_{\mathfrak{n o r m}, \mathfrak{a u x}, n-1}^{(h)}\right)^{2}}{2}, \mathrm{G}_{\mathfrak{n o r m}, \mathfrak{a u x}, i}^{(h)}, \cdots, \\
& \left.\mathrm{G}_{\mathfrak{n o r m}, \mathfrak{a u x}, n}^{(h)}, \Lambda_{\mathfrak{n o r m}, \mathfrak{a u x}, i}^{(h)}, \cdots, \Lambda_{\mathfrak{n o r m}, \mathfrak{a u x}, n}^{(h)}\right)
\end{aligned}
$$

and $f_{\mathfrak{e x p}, \mathfrak{s e c}, \mathfrak{a u x}, h}$ satisfies the bound for $f_{\mathfrak{e x p}, \mathfrak{s e c}, h}$ in (5.76). This will conclude the proof, up to apply the inverse transformation of (5.104), with $\mathrm{G}_{\mathfrak{a u x}, i}^{(h)}, \mathrm{g}_{\mathfrak{a u x}, i}^{(h)}, \chi_{*, i}^{(h)}$, $\kappa_{*, i}^{(h)}$ replaced by $\mathrm{G}_{\mathfrak{n o r m}, \mathfrak{a u x}, i}^{(h)}, \mathrm{g}_{\mathfrak{n o r m}, \mathfrak{a u x}, i}^{(h)}, \chi_{i}^{(h)}, \kappa_{i}^{(h)}$, and to take

$$
\mathfrak{D}_{\mathfrak{s e c}}^{h}:=\phi_{\mathfrak{a u x}}^{n-h}\left(\mathfrak{D}_{\mathfrak{s e c}, \mathfrak{a u x}}^{h}\right)
$$

We shall obtain the transformation $\phi_{\mathfrak{n o r m}, \mathfrak{a u x}}^{n-h}$ in (5.110) via an application of Proposition D.1. Before doing it, we just remark that, since, in our particular case, $f_{\mathfrak{n o r m}, \mathfrak{i n t}, \mathfrak{a} \mathfrak{u x}, h-1}$ depends on $\mathrm{z}_{\mathfrak{a u x}}^{(h)}, \mathrm{y}_{\mathfrak{a} \mathfrak{u x}}^{(h)}, \mathrm{x}_{\mathfrak{a} \mathfrak{u x}}^{(h)}$ only via $\mathrm{z}_{\mathfrak{a u x}, n-h}^{(h)}, \tilde{\mathrm{y}}_{\mathfrak{a} \mathfrak{u} \mathfrak{x}}^{(h)}, \hat{\mathrm{x}}_{\mathfrak{a} \mathfrak{u x}}^{(h)}$ and is even in $\left(\mathrm{t}_{\mathfrak{a u x}}^{(h)}, \mathrm{z}_{\mathfrak{a u x}, n-h}^{(h)}\right)$, the proof of Proposition D.1 can be easily handled to show that 
$\phi_{\mathfrak{n o r m}, \mathfrak{a u x}}^{n-h}$ can be chosen of the form

$$
\begin{aligned}
& \phi_{\mathfrak{n o r m}, \mathfrak{a u x}}^{n-h}:
\end{aligned}
$$

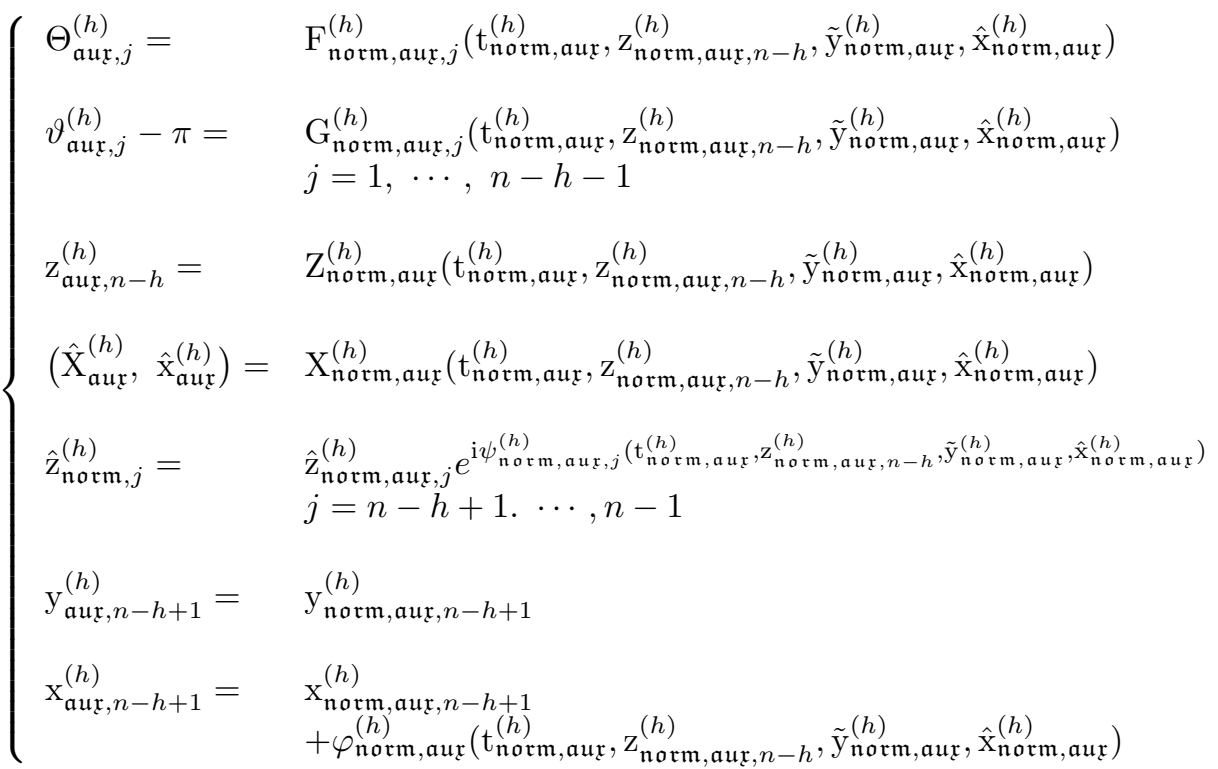

where $\mathrm{F}_{\mathfrak{n o r m}, \mathfrak{a u x}}^{(h)}, \mathrm{G}_{\mathfrak{n o r m}, \mathfrak{a u x}}^{(h)}$ and $\mathrm{Z}_{\mathfrak{n o r m}, \mathfrak{a u x}}^{(h)}$ are odd; $\mathrm{X}_{\mathfrak{n o r m}, \mathfrak{a u x}}^{(h)}, \quad \psi_{\mathfrak{n o r m}, \mathfrak{a u x}, j}^{(h)}$ and $\varphi_{\mathfrak{n o r m}, \mathfrak{a u x}}^{(h)}$ are even under the change

$$
\left(\mathrm{t}_{\mathfrak{n o r m}, \mathfrak{a u x}}^{(h)}, \mathrm{z}_{\mathfrak{n o r m}, \mathfrak{a u x}, n-h}^{(h)}\right) \rightarrow-\left(\mathrm{t}_{\mathfrak{n o r m}, \mathfrak{a u x}}^{(h)}, \mathrm{z}_{\mathfrak{n o r m}, \mathfrak{a u x}, n-h}^{(h)}\right) .
$$

Then (5.88)-(5.90) follow.

Now we proceed with proving the existence of $\phi_{\mathfrak{n o r m}, \mathfrak{a} \mathfrak{u x}}^{n-h}$. We can choose, in (D.133), (D.135) and (D.136),

$$
\begin{aligned}
& \nu_{i}=2(h+1), \quad \ell_{i}=h, \quad m_{i}=3 i, \quad i=1, \cdots, n-h-1=N \\
& \mathrm{~h}(p, q, I)=\sum_{i=n-h}^{n-1} \mathrm{~h}_{\mathfrak{s e c}}^{i}\left(\hat{\mathrm{y}}_{i}^{(h)}\right), \quad f(p, q, I, \varphi, \eta, \xi)=\sum_{i=1}^{n-h-1} f^{i}\left(u_{i}, p, q, \varphi\right) \\
& \left.f^{i}\left(u_{i}, p, q, \varphi\right):=f_{\mathfrak{n o r m}, \mathfrak{i n t}, \mathfrak{a} \mathfrak{a}, h-1}^{n-h-i} \mathrm{t}_{\mathfrak{a u x}, n-h-i}^{(h)}, \tilde{\mathrm{y}}_{\mathfrak{a u x}, n-h-i}^{(h)}, \hat{\mathrm{x}}_{\mathfrak{a u x}, n-h-i}^{(h)}\right) \\
& \mathfrak{Z}:=\mathfrak{Z}_{i}:=\left\{\left(k^{\prime}, k^{\prime \prime}, k^{\prime \prime \prime}\right) \in \mathbb{Z}^{h} \times \mathbb{Z}^{h+1} \times \mathbb{Z}^{h+1}: \quad k_{n-h+1}^{\prime}=\cdots=k_{n-1}^{\prime}=0\right. \\
& \left.k_{n-h+1}^{\prime \prime}=\cdots=k_{n}^{\prime \prime}=0, k_{n-h}^{\prime \prime \prime}=\cdots=k_{n}^{\prime \prime \prime}=0, k_{1}^{\prime \prime}+\cdots+k_{n-h}^{\prime \prime}=0\right\} \\
& \mathfrak{L}:=\left\{\left(k^{\prime}, k^{\prime \prime}, k^{\prime \prime \prime}\right) \in \mathfrak{Z}: \quad k_{n-h}^{\prime}=k_{n-h}^{\prime \prime}=0\right\}
\end{aligned}
$$


where we have re-named

$$
\begin{aligned}
(p, q) & :=\left(p_{\mathfrak{a u x}}^{(h)}, q_{\mathfrak{a u x}}^{(h)}\right)=\left(p_{\mathfrak{a u x}, n-h}^{(h)}, \cdots, p_{\mathfrak{a u x}, n-1}^{(h)}, q_{\mathfrak{a} \mathfrak{x}, n-h}^{(h)}, \cdots, q_{\mathfrak{a u x}, n-1}^{(h)},\right) \\
I & :=\left(\mathrm{G}_{\mathfrak{a u x}, n-h}^{(h)}, \cdots, \mathrm{G}_{\mathfrak{a u x}, n}^{(h)}, \Lambda_{\mathfrak{a u x}, n-h}^{(h)}, \cdots, \Lambda_{\mathfrak{a u x}, n}^{(h)}\right) \\
\varphi & :=\left(\mathrm{g}_{\mathfrak{a u x}, n-h}^{(h)}, \cdots, \mathrm{g}_{\mathfrak{a u x}, n}^{(h)}, \ell_{\mathfrak{a} \mathfrak{u}, n-h}^{(h)}, \cdots, \ell_{\mathfrak{a u x}, n}^{(h)}\right) \\
u_{i} & :=\left(I, \eta^{i}, \xi^{i}\right), \quad \eta:=\eta^{1}, \quad \xi:=\xi^{1}
\end{aligned}
$$

with

$$
\begin{aligned}
\eta^{i}:= & \left(\Theta_{\mathfrak{a u x}, n-h-i}, \cdots, \Theta_{\mathfrak{a u x}, n-1}, \mathrm{G}_{\mathfrak{a u x}, n-h-i}, \cdots, \mathrm{G}_{\mathfrak{a u x}, n-h-1},\right. \\
& \left.\Lambda_{\mathfrak{a u x}, n-h-i}, \cdots, \Lambda_{\mathfrak{a u x}, n-h-1}\right) \\
\xi^{i}:= & \left(\vartheta_{\mathfrak{a u x}, n-h-i}, \cdots, \vartheta_{\mathfrak{a u x}, n-1}, \mathrm{~g}_{\mathfrak{a u x}, n-h-i}, \cdots, \mathrm{g}_{\mathfrak{a u x}, n-h-1},\right. \\
& \left.\ell_{\mathfrak{a u x}, n-h-i}, \cdots, \ell_{\mathfrak{a u x}, n-h-1}\right) .
\end{aligned}
$$

In order to verify that Proposition D.1 can be applied, we have to check conditions (D.134) and (D.139). Due to the choices of $\mathfrak{Z}, \mathfrak{L}$ and to the fact that only the function $h_{\mathfrak{s e c}}^{n-h}$ in the summand for $h_{\mathfrak{s e c}}$ in (5.108) depends on

$$
\left(p_{\mathfrak{a u x}, n-h}^{(h)}, q_{\mathfrak{a u x}, n-h}^{(h)}, \mathrm{G}_{\mathfrak{a u x}, n-h}^{(h)}\right),
$$

it is sufficient to check that condition (D.134) holds with

$$
\omega=\omega_{\mathfrak{s e c}}^{n-h}, \quad\left(k^{\prime}, k\right) \in \mathbb{Z}^{2} \backslash\{0\}, \quad K=\bar{K} .
$$

But due to the choice of $\mathfrak{D}_{\mathfrak{i n t}, \mathfrak{a u x}}^{h}$ in (5.107), we have that (D.134) is verified, with

$$
\mathfrak{a}=\frac{\left(a_{n-h}^{+}\right)^{2}}{\left(a_{n-h+1}^{-}\right)^{3} \theta_{n-h}} \frac{\bar{\gamma}}{\bar{K}^{\bar{\tau}}}, \quad r=\mathfrak{c}_{h} \frac{\theta_{n-h} \bar{\gamma}}{\bar{K}^{\bar{\tau}+1}}, \quad \varepsilon=\mathfrak{c}_{h} \sqrt{\theta_{n-h}} .
$$

It remains to check the inequalities in (D.139). In view of the definition of $f^{i}$ following from the formulae (5.84), (5.109) and (5.111), of the definition of $f_{\mathfrak{n o r m}, h-1}^{i}$ in (5.74), the definition of $\overline{f_{\mathfrak{n o r m}, h-1}^{i}}$, the bound for $f_{\mathfrak{n o r m}, h-1}^{i}$ in (5.76), and first inequality in (4.54), we see that the former of the inequalities in (D.139) is satisfied with

$$
E_{i}=\frac{1}{\mathfrak{c}_{h}} \max \left\{\frac{\left(a_{n-h-i}^{+}\right)^{2}}{\left(a_{n-h-i+1}^{+}\right)^{3}}, \mu \bar{K}\left(\frac{a_{n}^{+}}{a_{1}^{-}}\right)^{\frac{3}{2}} \frac{1}{a_{n-h-i+1}^{-}}\right\} \quad i=1, \cdots, n-h-1 .
$$

In order to check that also the second inequality in (D.139) is satisfied, we previously note that the number $d_{i}$ in (D.138) can be taken to be

$$
d_{i}=\mathfrak{c}_{h} \min \left\{\frac{\theta_{n-h} \bar{\gamma}}{\bar{K}^{\bar{\tau}+1}}, \theta_{n-h-i}\right\}, \quad i=1, \cdots, n-h-1 .
$$

Inserting then the above values for $K, \mathfrak{a}, E_{i}$ and $d_{i}$ into the left hand side of the second inequality in (D.139), we find that this can be bounded by 


$$
\begin{gathered}
\frac{1}{\tilde{\mathfrak{c}}_{h}} \max \left\{\frac{\bar{K}^{2 \bar{\tau}+2}}{\bar{\gamma}^{2}} \frac{\left(a_{n-h-i}^{+}\right)^{2}}{\left(a_{n-h}^{+}\right)^{2}} \frac{\left(a_{n-h+1}^{-}\right)^{3}}{\left(a_{n-h-i+1}^{-}\right)^{3}}, \quad \frac{\bar{K}^{\bar{\tau}+1}}{\bar{\gamma}} \frac{\left(a_{n-h-i}^{+}\right)^{2}}{\left(a_{n-h}^{+}\right)^{2}} \frac{\left(a_{n-h+1}^{-}\right)^{3}}{\left(a_{n-h-i+1}^{-}\right)^{3}} \frac{\theta_{n-h}}{\theta_{n-h-i}}\right. \\
\left.\frac{\bar{K}^{2 \bar{\tau}+2}}{\bar{\gamma}^{2}} \frac{\mu \bar{K}\left(\frac{a_{n}^{+}}{a_{1}^{-}}\right)^{\frac{3}{2}}}{\left(a_{n-h}^{+}\right)^{2}} \frac{\left(a_{n-h+1}^{-}\right)^{3}}{a_{n-h-i+1}^{-}}, \quad \frac{\bar{K}^{\bar{\tau}+1}}{\bar{\gamma}} \frac{\mu \bar{K}\left(\frac{a_{n}^{+}}{a_{1}^{-}}\right)^{\frac{3}{2}}}{\left(a_{n-h}^{+}\right)^{2}} \frac{\left(a_{n-h+1}^{-}\right)^{3}}{a_{n-h-i+1}^{-}} \frac{\theta_{n-h}}{\theta_{n-h-i}}\right\}
\end{gathered}
$$

Using (4.50), one easily finds that this quantity does not exceed

$$
\frac{1}{\hat{\mathfrak{c}}_{h}} \max \left\{\mu\left(\frac{a_{n}}{a_{1}}\right)^{5} \frac{\bar{K}^{2 \bar{\tau}+2}}{\bar{\gamma}^{2}}, \quad \frac{\bar{K}^{\bar{\tau}+1} \sqrt{\alpha}}{\bar{\gamma}}\right\}<1 .
$$

where $\hat{\mathfrak{c}}_{h}$ depends only on the ratio $a_{n}^{-} / a_{n}^{+}$and the masses and the inequality follows from (4.54). This conclude the proof of this case.

5.2.4. Construction of $\phi_{\mathfrak{n o r m}}^{n-1}$. The arguments we have used in the previous section to construct $\phi_{\mathfrak{n o r m}}^{1}, \cdots, \phi_{\mathfrak{n o r m}}^{n-2}$ also fit for the case of $\phi_{\mathfrak{n o r m}}^{n-1}$, therefore we shall not repeat them. We only limit to remark that, for this case, Equations (5.106), (5.111), (5.112) and (5.113) have to be replaced with

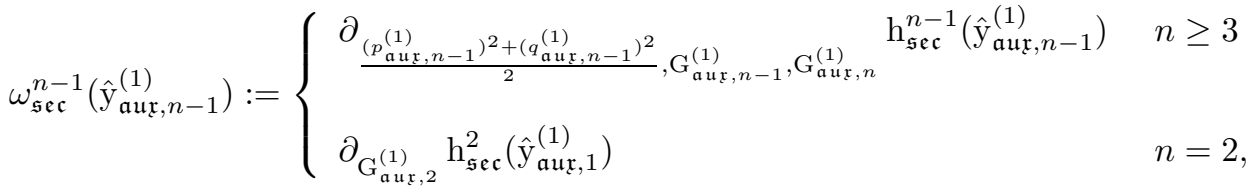

$$
\begin{aligned}
& f^{i}=f_{\mathfrak{n o r m}, \mathfrak{i n t}, \mathfrak{a u x}, 0}^{n-i}\left(\mathrm{t}_{\mathfrak{a u x}, i}^{(1)}, \hat{\mathrm{y}}_{\mathfrak{a u x}, i}^{(1)}, \hat{\mathrm{x}}_{\mathfrak{a u x}, i}^{(1)}\right), \quad d_{i}=\mathfrak{c}_{1} \min \left\{\frac{\theta_{n-1} \bar{\gamma}}{\bar{K}^{\bar{\tau}+1}}, \theta_{n-i-1}\right\} \\
& i=1, \cdots, n-1, \quad \theta_{0}:=\theta_{1} \\
& E_{i}=\frac{1}{\hat{\mathfrak{c}}_{1}} \begin{cases}\max \left\{\mu\left(\frac{a_{n}^{+}}{a_{1}^{-}}\right)^{\frac{3}{2}} \frac{1}{a_{n}^{-}}, \frac{\left(a_{n-1}^{+}\right)^{3}}{\left(a_{n}^{-}\right)^{4}}\right\} & i=1 \\
\max \left\{\mu \bar{K}\left(\frac{a_{n}^{+}}{a_{1}^{-}}\right)^{\frac{3}{2}} \frac{1}{a_{n-i+1}^{-}}, \frac{\left(a_{n-i}^{+}\right)^{2}}{\left(a_{n-i+1}^{-}\right)^{3}}\right\} & n \geq 3, i=2, \cdots, n-1\end{cases} \\
& \frac{1}{\hat{\mathfrak{c}}_{1}} \max \left\{\mu\left(\frac{a_{n}}{a_{1}}\right)^{5} \frac{\bar{K}^{2 \bar{\tau}+2}}{\bar{\gamma}^{2}}, \quad \frac{\bar{K}^{2(\bar{\tau}+1)} \alpha}{\bar{\gamma}^{2}}\right\} \text {. }
\end{aligned}
$$




\section{APPENDIX A}

\section{Computing the domain of holomorphy}

\section{A.1. On the analyticity of the solution of Kepler equation}

Here is a refinement of Proposition 4.1 .

Proposition A.1. Let $\widehat{e}$ be as in (4.47). For any $0<\bar{e}<\widehat{e}$ there exists $\bar{\eta}=\bar{\eta}(\bar{e})$ such that, for any $\bar{\eta}<\eta<1$ and any $\mathrm{e} \in \mathbb{C}$ with $|\mathrm{e}| \leq \bar{e}$, there exist two positive numbers $\bar{\zeta}=\bar{\zeta}(\eta, \mathrm{e}), \bar{\ell}=\bar{\ell}(\eta, \bar{e})$ such that the map

$$
\zeta \in \overline{\mathbb{T}}_{\bar{\zeta}} \rightarrow K(\zeta, \mathrm{e}):=\zeta-\mathrm{e} \sin \zeta
$$

is injective, its image verifies

$$
K\left(\overline{\mathbb{T}}_{\bar{\zeta}}, \mathrm{e}\right) \supset \overline{\mathbb{T}}_{\bar{\ell}} \quad \forall \mathrm{e} \in \mathbb{C}:|\mathrm{e}| \leq \bar{e} .
$$

The inverse function

$$
\ell \in \overline{\mathbb{T}}_{\bar{\ell}} \rightarrow \zeta(\ell, \mathrm{e}):=K^{-1}(\ell, \mathrm{e}) \in \overline{\mathbb{T}}_{\bar{\zeta}_{\eta}(\mathrm{e})}
$$

verifies

$$
|1-\mathrm{e} \cos \zeta(\ell, \mathrm{e})| \geq 1-\eta
$$

Therefore, $\zeta(\ell, \mathrm{e})$ is real-analytic for $\ell \in \overline{\mathbb{T}}_{\bar{\ell}}$.

The proof of Proposition A.1 is elementary and goes along the same lines of [24]. Therefore, we shall present it skipping some detail.

Lemma A.1. Let $\widehat{e}$ be as in Proposition 4.1. For any $0<\bar{e}<\widehat{e}$ there exists a unique $\bar{\eta}=\bar{\eta}(\bar{e}) \in(\bar{e}, 1)$ such that

$$
\begin{gathered}
\forall \eta \in[\bar{\eta}, 1): \quad \bar{\ell}_{\eta}(\bar{e}):=\log \left[\frac{\eta}{\bar{e}}+\sqrt{1+\frac{\eta^{2}}{\bar{e}^{2}}}\right]-\sqrt{\eta^{2}+\bar{e}^{2}} \geq 0, \\
\ell_{\eta}(\bar{e})=0 \quad \Longleftrightarrow \quad \eta=\bar{\eta} .
\end{gathered}
$$

Proof. By definition of $\widehat{e}$, and since the function $\rho \in[0,1] \rightarrow \frac{\rho e^{\sqrt{1+\rho^{2}}}}{1+\sqrt{1+\rho^{2}}}$ increases with $\rho$, we have

Consider now the function

$$
\frac{\bar{e} e^{\sqrt{1+\bar{e}^{2}}}}{1+\sqrt{1+\bar{e}^{2}}}<1
$$

$$
\eta \in(0,1] \rightarrow g_{\rho}(\eta):=\frac{\rho e^{\sqrt{\eta^{2}+\rho^{2}}}}{\eta+\sqrt{\eta^{2}+\rho^{2}}} .
$$

This function decreases with $\eta$ for any $\rho \in(0,1]$. Since

$$
g_{\bar{e}}(0)=e^{\bar{e}}>1, \quad g_{\bar{e}}(1)=\frac{\bar{e} e^{\sqrt{1+\bar{e}^{2}}}}{1+\sqrt{1+\bar{e}^{2}}}<1
$$


we find a unique $\bar{\eta}=\bar{\eta}(\bar{e}) \in[0,1]$ such that

$$
g_{\bar{e}}(\eta)<1 \quad \forall \bar{\eta}<\eta<1, \quad g_{\bar{e}}(\eta(\bar{e}))=1 .
$$

Since also

we actually have

$$
g_{\bar{e}}(\bar{e})=\frac{e^{\bar{e} \sqrt{2}}}{1+\sqrt{2}} \geq \frac{e^{\sqrt{2}}}{1+\sqrt{2}}>1
$$

$$
\bar{e}<\bar{\eta}<1 \text {. }
$$

Proof of Proposition A.1. We shall prove Proposition A.1 with

$$
\begin{aligned}
& \bar{\zeta}(\eta, \mathrm{e}):=\log \frac{\sqrt{\eta^{2}+\mathrm{e}_{2}^{2}}+\sqrt{\eta^{2}-\mathrm{e}_{1}^{2}}}{\sqrt{\mathrm{e}_{1}^{2}+\mathrm{e}_{2}^{2}}} \\
& \bar{\ell}(\eta, \bar{e}):=\log \left[\frac{\eta}{\bar{e}}+\sqrt{1+\frac{\eta^{2}}{\bar{e}^{2}}}\right]-\sqrt{\eta^{2}+\bar{e}^{2}}
\end{aligned}
$$

where $\mathrm{e}=\mathrm{e}_{1}+\mathrm{ie}_{2}$. Observe that $\bar{\ell}(\eta, \bar{e})>0$ by Lemma A.1. Moreover, since

$$
\mathrm{e}_{1} \leq|\mathrm{e}| \leq \bar{e}<\bar{\eta}<\eta
$$

we have that $\bar{\zeta}(\eta$, e) is well defined and positive1:

$$
\bar{\zeta}(\eta, \mathrm{e}) \geq \log \frac{\eta}{\bar{e}}>0 .
$$

We split Equation (A.114) into its real and imaginary part

$$
\left\{\begin{array}{l}
K_{1}\left(\zeta_{1}, \zeta_{2}, \mathrm{e}_{1}, \mathrm{e}_{2}\right):=\zeta_{1}-\left(\mathrm{e}_{1} \sin \zeta_{1} \cosh \zeta_{2}-\mathrm{e}_{2} \cos \zeta_{1} \sinh \zeta_{2}\right)=\ell_{1} \\
K_{2}\left(\zeta_{1}, \zeta_{2}, \mathrm{e}_{1}, \mathrm{e}_{2}\right):=\zeta_{2}-\left(\mathrm{e}_{1} \cos \zeta_{1} \sinh \zeta_{2}+\mathrm{e}_{2} \sin \zeta_{1} \cosh \zeta_{2}\right)=\ell_{2}
\end{array}\right.
$$

(with $\zeta=\zeta_{1}+\mathrm{i} \zeta_{2}, \ell=\ell_{1}+\mathrm{i} \ell_{2}$ ). The equation for the real part gives a unique solution

provided

$$
\zeta_{1}=\mathrm{Z}_{1}\left(\mathrm{e}_{1}, \mathrm{e}_{2}, \zeta_{2}, \ell_{1}\right)
$$

$$
\left|\mathrm{e}_{1}\right| \leq \eta, \quad\left|\zeta_{2}\right| \leq \bar{\zeta}(\eta, \mathrm{e})
$$

since it reduces to an ordinary real Kepler equation

$$
\begin{gathered}
\zeta_{1}-E_{1}\left(\mathrm{e}_{1}, \mathrm{e}_{2}, \zeta_{2}\right) \sin \left(\zeta_{1}-\phi_{1}\left(\mathrm{e}_{1}, \mathrm{e}_{2}, \zeta_{2}\right)=\ell_{1} \quad \text { if } \quad E_{1}\left(\mathrm{e}_{1}, \mathrm{e}_{2}, \zeta_{2}\right) \neq 0\right. \\
\zeta_{1}=\ell_{1} \quad \text { otherwise }
\end{gathered}
$$

with

$$
\begin{aligned}
& E_{1}\left(\mathrm{e}_{1}, \mathrm{e}_{2}, \zeta_{2}\right):=\sqrt{\mathrm{e}_{1}^{2} \cosh ^{2} \zeta_{2}+\mathrm{e}_{2}^{2} \sinh ^{2} \zeta_{2}} \\
& \phi_{1}\left(\mathrm{e}_{1}, \mathrm{e}_{2}, \zeta_{2}\right): \quad E_{1} \cos \phi_{1}=\mathrm{e}_{1} \cosh \zeta_{2}, \quad E_{1} \sin \phi_{1}=\mathrm{e}_{2} \sinh \zeta_{2} .
\end{aligned}
$$

and, under condition (A.117), one has

$$
E_{1} \leq \eta<1
$$

${ }^{1}$ Actually, $\bar{\zeta}(\eta, \mathrm{e})$, as a function of $\left(\mathrm{e}_{1}, \mathrm{e}_{2}\right)$, reaches its positive minimum

$$
\bar{\zeta}_{\text {min }}=\log \left[\frac{\eta}{\bar{e}}+\sqrt{1+\frac{\eta^{2}}{\bar{e}^{2}}}\right]>\log (1+\sqrt{2})
$$

for $\left(\mathrm{e}_{1}, \mathrm{e}_{2}\right)=(0, \bar{e})$. 
Observe that this solution $\mathrm{Z}_{1}\left(\mathrm{e}_{1}, \mathrm{e}_{2}, \zeta_{2}, \ell_{1}\right)$ verifies

$$
\mathrm{Z}_{1}\left(\mathrm{e}_{1}, \mathrm{e}_{2},-\zeta_{2}, \ell_{1}\right)=-\mathrm{Z}_{1}\left(\mathrm{e}_{1}, \mathrm{e}_{2}, \zeta_{2}, \ell_{1}\right) \quad \bmod 2 \pi .
$$

On the other hand, the function

$$
\zeta_{2} \rightarrow \mathrm{K}_{2}\left(\mathrm{e}_{1}, \mathrm{e}_{2}, \zeta_{2}, \ell_{1}\right):=K_{2}\left(\mathrm{Z}_{1}\left(\mathrm{e}_{1}, \mathrm{e}_{2}, \zeta_{2}, \ell_{1}\right), \zeta_{2}, \mathrm{e}_{1}, \mathrm{e}_{2}\right)
$$

is strictly increasing, therefore, it maps the interval $[-\bar{\zeta}(\eta, \mathrm{e}), \bar{\zeta}(\eta, \mathrm{e})]$, onto the interval $\left[-\mathrm{L}_{2}\left(\eta, \mathrm{e}, \ell_{1}\right), \mathrm{L}_{2}\left(\eta, \mathrm{e}, \ell_{1}\right)\right]$, where $\mathrm{L}_{2}\left(\eta, \mathrm{e}, \ell_{1}\right):=\mathrm{K}_{2}\left(\mathrm{e}_{1}, \mathrm{e}_{2}, \bar{\zeta}(\eta, \mathrm{e}), \ell_{1}\right)$ (note that $\mathrm{K}_{2}\left(\mathrm{e}_{1}, \mathrm{e}_{2},-\bar{\zeta}(\eta, \mathrm{e}), \ell_{1}\right)=-\mathrm{K}_{2}\left(\mathrm{e}_{1}, \mathrm{e}_{2}, \bar{\zeta}(\eta, \mathrm{e}), \ell_{1}\right)$ because of A.119) $)$. We have thus proved that the map (A.114) maps bijectively the strip $\overline{\mathbb{T}}_{\bar{\zeta}(\eta, \mathrm{e})}$ onto the set

$$
\ell=\ell_{1}+\mathrm{i} \ell_{2} \in \mathbb{C}: \quad \ell_{1} \in \mathbb{T}, \quad \ell_{2} \in\left[-\mathrm{L}_{2}\left(\eta, \mathrm{e}, \ell_{1}\right), \mathrm{L}_{2}\left(\eta, \mathrm{e}, \ell_{1}\right)\right] .
$$

But the curve

$$
\ell_{2}=\mathrm{L}_{2}\left(\eta, \mathrm{e}, \ell_{1}\right) \quad \ell_{1} \in[0,2 \pi)
$$

is concave, its minimum points are cusps, where $\mathrm{L}_{2}$ attains the value

$$
\mathrm{L}_{2, \min }(\eta, \mathrm{e})=\bar{\zeta}(\eta, \mathrm{e})-\sqrt{\eta^{2}-\mathrm{e}_{1}^{2}+\mathrm{e}_{2}^{2}}
$$

The minimum of this quantity while $|\mathrm{e}| \leq \bar{e}$ is just $\bar{\ell}(\eta, \bar{e})$ in (A.116). Inequality in (A.115) follows from

$$
|1-\mathrm{e} \cos \zeta| \geq|\operatorname{Re}(1-\mathrm{e} \cos \zeta)| \geq 1-|\operatorname{Re}(\mathrm{e} \cos \zeta)|
$$

and (by (A.118)

$$
|\operatorname{Re}(\mathrm{e} \cos \zeta)|=\mid E_{1}\left(\mathrm{e}_{1}, \mathrm{e}_{2}, \zeta_{2}\right) \cos \left(\zeta_{1}-\phi_{1}\left(\mathrm{e}_{1}, \mathrm{e}_{2}, \zeta_{2}\right) \mid \leq E_{1} \leq \eta .\right.
$$

\section{A.2. Proof of Proposition 4.2}

Define

$$
\underline{\delta}_{j}:=\sqrt{1-\bar{e}_{j}^{2}}, \quad \bar{\delta}_{j}:=\sqrt{1-\underline{e}_{j}^{2}} .
$$

Assume (4.48), with

$$
\begin{aligned}
& \mathrm{A}:=\left(1-\sigma^{2}\right) \sqrt{\frac{1}{(1+\sigma)^{3}\left(1+\sigma^{2}\right)^{4}}}, \quad \mathcal{B}:=\sqrt{\frac{1}{\left(1-\sigma^{2}\right)(1+\sigma)^{3}\left(1+\sigma^{2}\right)}} \\
& \overline{\mathrm{C}}_{i}:=\left\{\begin{array}{l}
\mathrm{C}_{1}(\sigma) \bar{\delta}_{i} \quad i=1, \cdots, n-1 \\
\bar{\delta}_{n} \quad i=n
\end{array},\right. \\
& \underline{\mathrm{C}}_{i}:=\left\{\begin{array}{l}
\mathrm{C}_{2}(\sigma) \sqrt{\underline{\delta}_{i}^{2}+2 g(\sigma)^{2} \bar{\delta}_{i}^{2}} \quad i=1, \cdots, n-1 \\
\sqrt{\underline{\delta}_{i}^{2}+2 g(\sigma)^{2} \bar{\delta}_{n}^{2}} \quad i=n
\end{array}\right. \\
& s=\sigma(1-\sigma)
\end{aligned}
$$

where

$$
\mathrm{C}_{1}(\sigma):=\sqrt{1-\sigma^{2}}, \quad \mathrm{C}_{2}(\sigma):=\sqrt{\frac{\left(1+\sigma^{2}\right)^{3}}{\left(1-\sigma^{2}\right)^{2}}}
$$


and $\sigma, g$ are chosen as follows: $g\left(\sigma^{\prime}\right)$ is a suitable positive function, depending at most on the ratios $\frac{\Lambda_{j}^{+}}{\Lambda_{j}^{-}}, \frac{\mathrm{G}_{i}^{+}}{\mathrm{G}_{i}^{-}}, \operatorname{such}^{2}$ that

$$
g\left(\sigma^{\prime}\right) \rightarrow 0 \quad \text { as } \quad \sigma^{\prime} \rightarrow 0, \quad \text { and } \quad\left|\sin \arg \frac{\left\|\mathrm{C}_{\mathcal{P}}^{(j)}\right\|^{2}}{\Lambda_{j}^{2}}\right| \leq g\left(\sigma^{\prime}\right), \quad j=1, \cdots, n,
$$

provided

$$
\max \left\{\left|\arg \left(\Lambda_{i}\right)\right|,\left|\arg \left(\chi_{j}\right)\right|,\left|\arg \left(\Theta_{j}\right)\right|,\left|\arg \left(\vartheta_{j}\right)\right|\right\} \leq \sigma^{\prime}
$$

while $\sigma$ is so small that, if $\bar{\ell}_{1}, \cdots, \bar{\ell}_{n}$ are as in Proposition 4.1, with $\bar{e}$ replaced by $\bar{e}_{1}, \cdots, \bar{e}_{n}$, then

and the following inequality is satisfied

$$
\sigma \leq \min \left\{\frac{3}{4}, \bar{\ell}_{1}, \cdots, \bar{\ell}_{n}\right\}
$$

$$
\frac{\mathrm{C}_{1}(\sigma)}{\mathrm{C}_{2}(\sigma)} \frac{\bar{\delta}_{j}}{\sqrt{\underline{\delta}_{j}^{2}+\sqrt{2} g(\sigma) \bar{\delta}_{j}}}>1 \quad \forall i=1, \cdots, n .
$$

Note that this inequality is satisfied for $\sigma$ suitably small, since, by definition,

$$
\bar{\delta}_{j}>\underline{\delta}_{j}, \quad \mathrm{C}_{1}\left(\sigma^{\prime}\right) \uparrow 1, \quad \mathrm{C}_{2}\left(\sigma^{\prime}\right) \downarrow 1, \quad g\left(\sigma^{\prime}\right) \downarrow 0 \quad \text { as } \quad \sigma^{\prime} \rightarrow 0 .
$$

Definitions and assumptions in (4.48) imply, since $\sigma(1-\sigma)<\sigma$,

$$
\begin{aligned}
& (1-\sigma) \mathrm{G}_{n}^{-}<\left|\chi_{i}\right|<\mathrm{G}_{n}^{+}(1+\sigma) \\
& \left|\tan \arg \left(\chi_{i-1}-\chi_{i}\right)\right| \leq \frac{\max \left|\operatorname{Im}\left(\chi_{i-1}-\chi_{i}\right)\right|}{\min \left|\operatorname{Re}\left(\chi_{i-1}-\chi_{i}\right)\right|} \leq \frac{\theta_{i}}{\mathrm{G}_{i}^{-}} \leq \sigma \leq 1 \\
& \left|\arg \chi_{i}\right| \leq\left|\arg \chi_{n-1}\right|+\sum_{j=i+1}^{n-1}\left|\sin ^{-1} \frac{\left|\chi_{j-1}-\chi_{j}\right|}{\left|\chi_{j}-\chi_{j+1}\right|}\right| \leq \sigma \leq \frac{\pi}{3}
\end{aligned}
$$

The previous inequalities imply that, firstly

$$
\left|\frac{\Theta_{j}}{\chi_{j-1}}\right| \leq \frac{\sigma(1-\sigma) \mathrm{G}_{n}^{-}}{(1-\sigma) \mathrm{G}_{n}^{-}} \leq \sigma
$$

and, similarly,

$$
\left|\frac{\Theta_{j}}{\chi_{j}}\right| \leq \sigma
$$

therefore, the inequality for $\mathrm{i}_{j}, \iota_{i}$ is (4.49) follows. Secondly, the definitions of $\Theta_{i}^{+}$, $\vartheta_{i}^{+}$imply that conditions (A.127) are met and hence Lemma A.2 applies. By the thesis A.128, we have , for $j=1, \cdots, n-1$,

${ }^{2}$ Since, for $j=1, \cdots, n,\left\|\mathrm{C}_{\mathcal{P}}^{(j)}\right\|^{2}$ depends only on $\chi_{j-1}, \chi_{j}, \Theta_{j}$ and $\vartheta_{j}$ as in 2.17) and all such coordinates, together also with $\Lambda_{j}$, have their anomalies bounded by $\sigma^{\prime}$, we can always find such a function $g\left(\sigma^{\prime}\right)$.

${ }^{3}$ Beware that, if $z=\left(z_{1}, z_{2}, z_{3}\right) \in \mathbb{C}^{3}$, we denote

$$
\|z\|^{2}:=z_{1}^{2}+z_{2}^{2}+z_{3}^{3} .
$$

For a given $z \in \mathbb{C}$, the symbol $|z|$ denotes the usual modulus of $z \in \mathbb{C}$ :

$$
|z|:=\sqrt{(\operatorname{Re} z)^{2}+(\operatorname{Im} z)^{2}} .
$$




$$
\begin{aligned}
\left|\left\|\mathrm{C}_{\mathcal{P}}^{(j)}\right\|^{2}\right| & \leq \frac{\left|\chi_{j-1}-\chi_{j}\right|^{2}}{1-\sigma^{2}}+(1+\sigma)\left(1+\sigma^{2}\right)\left|\chi_{j-1}\right|\left|\chi_{j}\right|\left|\vartheta_{j}-\pi\right|^{2} \\
& \leq \frac{\left(\mathrm{G}_{i}^{+}\right)^{2}}{\overline{\mathrm{C}}_{j}^{2}}+\frac{\left(\mathrm{G}_{n}^{+}\right)^{2}}{\overline{\mathrm{C}}_{j}^{2} \mathrm{~B}^{2}}\left|\vartheta_{j}-\pi\right|^{2} \\
& \leq \bar{\delta}_{j}^{2}\left(\Lambda_{j}^{-}\right)^{2} .
\end{aligned}
$$

For $j=n$,

$$
\left|\left\|\mathrm{C}_{\mathcal{P}}^{(j)}\right\|^{2}\right|=\left|\chi_{n-1}\right|^{2} \leq\left(\mathrm{G}_{n}^{+}\right)^{2}<\bar{\delta}_{n}^{2}\left(\Lambda_{n}^{-}\right)^{2} .
$$

We suddenly have the left bound in (4.49):

$$
1-\left|e_{i, \mathcal{P}}^{2}\right| \leq\left|1-e_{i, \mathcal{P}}^{2}\right|=\left|\frac{\left|\left\|\mathrm{C}_{\mathcal{P}}^{(i)}\right\|^{2}\right|}{\Lambda_{i}^{2}}\right| \leq \bar{\delta}_{i}^{2}=1-\underline{e}_{i}^{2},
$$

for $i=1, \cdots, n$. Now we check the right bound. To this end, previously check the following inequality

$$
|| \chi_{j-1}|-| \chi_{j}|| \geq \frac{1-\sigma^{2}}{1+\sigma^{2}} \mathrm{G}_{j}^{-}
$$

Because of the second inequality in (A.122),

$$
\left|\arg \left[\left(\chi_{j-1}-\chi_{j}\right)\left(\bar{\chi}_{m-1}-\bar{\chi}_{m}\right)\right]\right| \leq 2 \tan ^{-1} \sigma .
$$

Then we have

$$
\operatorname{Re}\left[\left(\chi_{j-1}-\chi_{j}\right)\left(\bar{\chi}_{m-1}-\bar{\chi}_{m}\right)\right] \geq \frac{1-\sigma^{2}}{1+\sigma^{2}}\left|\chi_{j-1}-\chi_{j}\right|\left|\bar{\chi}_{m-1}-\bar{\chi}_{m}\right| .
$$

Taking the sum for $m=j+1, \cdots, n$, gives

$$
\begin{aligned}
\operatorname{Re}\left(\chi_{j-1}-\chi_{j}\right) \bar{\chi}_{j} & \geq \frac{1-\sigma^{2}}{1+\sigma^{2}}\left|\chi_{j-1}-\chi_{j}\right| \sum_{m=j+1}^{n}\left|\bar{\chi}_{m-1}-\bar{\chi}_{m}\right| \\
& \geq \frac{1-s^{2}}{1+s^{2}}\left|\chi_{j-1}-\chi_{j}\right|\left|\bar{\chi}_{j}\right| \\
& \geq \frac{1-\sigma^{2}}{1+\sigma^{2}} \mathrm{G}_{j}^{-}\left|\bar{\chi}_{j}\right|
\end{aligned}
$$

So, Lemma A.3 with

$$
A=\chi_{j-1}, \quad B=\chi_{j}, \quad \Delta=\mathrm{G}_{j}^{-}, \quad a=\frac{1-\sigma^{2}}{1+\sigma^{2}}
$$

gives A.124). Then the thesis A.129 of Lemma A.2 and the definition of $\vartheta_{j}$ provide, for $j=1, \cdots, n-1$,

$$
\left|\left\|\mathrm{C}_{\mathcal{P}}^{(j)}\right\|^{2}\right| \geq \frac{1}{\mathcal{A}^{2} \underline{\mathcal{C}}_{j}^{2}}\left[\mathcal{A}^{2}\left(\mathrm{G}_{j}^{-}\right)^{2}-\left(\mathrm{G}_{n}^{+}\right)^{2}\left|\vartheta_{j}-\pi\right|^{2} \geq\right] \geq\left(\underline{\delta}_{j}^{2}+\sqrt{2} g(\sigma) \bar{\delta}_{i}\right)\left(\Lambda_{j}^{+}\right)^{2}
$$

where $g(\sigma)$ is as in (A.121). Again, this inequality is implied by the definition of $\vartheta_{j}^{+}$ in (4.48) and the ones of $\mathcal{A}$ and $\mathrm{C}_{2}$ in (A.120). By (A.121), (A.123) and (A.125), 
for $j=1, \cdots, n$, we have

$$
\begin{aligned}
\left|e_{j, \mathcal{P}}\right|^{2} & =\sqrt{\left(1-\operatorname{Re} \frac{\left\|\mathrm{C}_{\mathcal{P}}^{(j)}\right\|^{2}}{\Lambda_{j}^{2}}\right)^{2}+\left(\operatorname{Im} \frac{\left\|\mathrm{C}_{\mathcal{P}}^{(j)}\right\|^{2}}{\Lambda_{j}^{2}}\right)^{2}} \\
& \leq \sqrt{\left(1-\left|\frac{\left\|\mathrm{C}_{\mathcal{P}}^{(j)}\right\|^{2}}{\Lambda_{j}^{2}}\right|\right)^{2}+2\left|\operatorname{Im} \frac{\left\|\mathrm{C}_{\mathcal{P}}^{(j)}\right\|^{2}}{\Lambda_{j}^{2}}\right|} \\
& \leq \sqrt{\left(1-\underline{\delta}_{j}^{2}-\sqrt{2} g(\sigma) \bar{\delta}_{j}\right)^{2}+2 \bar{\delta}_{j}^{2} g(\sigma)^{2}} \leq 1-\underline{\delta}_{j}^{2}=\bar{e}_{j}^{2} .
\end{aligned}
$$

For $j=n$,

$$
\left|\left\|\mathrm{C}_{\mathcal{P}}^{(n)}\right\|^{2}\right|=\left|\chi_{n-1}\right|^{2} \geq\left(\underline{\delta}_{n}^{2}+\sqrt{2} g(\sigma) \bar{\delta}_{n}\right)\left(\Lambda_{n}^{+}\right)^{2}
$$

again implies (A.126) with $j=n$.

The proof of the inequality on the right in (4.49) proceeds in a similar way. Indeed, starting with

$$
\begin{aligned}
\left|d_{i, \mathcal{P}}\right|^{2}=\mid\left\|x_{\mathcal{P}}^{(i+1)}\right\|^{2}-2 x_{\mathcal{P}}^{(i)} \cdot x_{\mathcal{P}}^{(i+1)}+ & \left\|x_{\mathcal{P}}^{(i)}\right\|^{2} \mid \\
& \geq\left|\left\|x_{\mathcal{P}}^{(i+1)}\right\|^{2}\right|-2\left|x_{\mathcal{P}}^{(i)} \cdot x_{\mathcal{P}}^{(i+1)}\right|-\left|\left\|x_{\mathcal{P}}^{(i)}\right\|^{2}\right|
\end{aligned}
$$

and using (as it follows from Proposition A.1)

$$
\left|\left\|x_{\mathcal{P}}^{(i+1)}\right\|^{2}\right|=\left|a_{i+1}^{2}\left(1-e_{i+1, \mathcal{P}} \cos \zeta_{i+1}\right)^{2}\right| \geq\left(1-\eta_{i+1}\right)^{2}\left(a_{i+1}^{-}\right)^{2}
$$

and analogous arguments as above to evaluate $\left|x_{\mathcal{P}}^{(i)} \cdot x_{\mathcal{P}}^{(i+1)}\right|$ and $\left|\left\|x_{\mathcal{P}}^{(i)}\right\|^{2}\right|$, one easily finds the ansatz.

\section{Estimates.}

Lemma A.2. Fix a number $\sigma>0$. Assume that, for $1 \leq j \leq n-1$,

(A.127)

$\operatorname{Re} \bar{\chi}_{j}\left(\chi_{j-1}-\chi_{j}\right)>0, \quad\left|\Theta_{j}\right| \leq \sigma \min \left\{\left|\chi_{j-1}\right|, \quad\left|\chi_{j}\right|\right\}, \quad\left|\operatorname{Im}\left(\vartheta_{j}-\pi\right)\right| \leq \log (1+\sigma)$.

Then

$$
\begin{aligned}
\left|\left\|\mathrm{C}_{\mathcal{P}}^{(j)}\right\|^{2}\right| & \leq \frac{\left|\chi_{j-1}-\chi_{j}\right|^{2}}{1-\sigma^{2}}+(1+\sigma)\left(1+\sigma^{2}\right)\left|\chi_{j-1}\right|\left|\chi_{j} \| \vartheta_{j}-\pi\right|^{2} \\
\left|\left\|\mathrm{C}_{\mathcal{P}}^{(j)}\right\|^{2}\right| & \geq \frac{|| \chi_{j-1}|-| \chi_{j}||^{2}}{1+\sigma^{2}}-(1+\sigma)\left(1+\sigma^{2}\right)\left|\chi_{j-1} \| \chi_{j}\right|\left|\vartheta_{j}-\pi\right|^{2}
\end{aligned}
$$


Proof. We use the formula (2.19). By Taylor's, given $a, b, z \in \mathbb{C}$, with $|z| \leq \sigma \min _{t \in[0,1]}|a+t(b-a)|$

$$
\begin{aligned}
\left|\sqrt{b^{2}-z^{2}}-\sqrt{a^{2}-z^{2}}\right| & =\left|\int_{0}^{1} \frac{d}{d t} \sqrt{(a+t(b-a))^{2}-z^{2}} d t\right| \\
& =\left|(b-a) \int_{0}^{1} \frac{a+t(b-a)}{\sqrt{(a+t(b-a))^{2}-z^{2}}} d t\right| \\
& \leq|b-a| \int_{0}^{1} \frac{|a+t(b-a)|}{\sqrt{|a+t(b-a)|^{2}-|z|^{2}}} d t \\
& \leq \frac{|b-a|}{\sqrt{1-\sigma^{2}}}
\end{aligned}
$$

We use this formula with $b:=\chi_{j-1}, a:=\chi_{j}, z:=\Theta_{j}$, with the observation that, for $\operatorname{Re} \bar{\chi}_{j}\left(\chi_{j-1}-\chi_{j}\right)>0$, the function

$$
t \in[0,1] \rightarrow\left|\chi_{j}+t\left(\chi_{j-1}-\chi_{j}\right)\right|^{2}=\left|\chi_{j}\right|^{2}+2 t \operatorname{Re} \bar{\chi}_{j}\left(\chi_{j-1}-\chi_{j}\right)+t^{2}\left|\chi_{j-1}-\chi_{j}\right|^{2}
$$

reaches its minimum, given by $\min \left\{\left|\chi_{j-1}\right|^{2},\left|\chi_{j}\right|^{2}\right\}$, for $t=0$ or $t=1$. Developing also the function $w \in \mathbb{C} \rightarrow \cos w$ around $w=\pi$, with $\varrho:=w-\pi=\varrho_{1}+\mathrm{i} \varrho_{2}$ and $\left|\rho_{2}\right| \leq \log (1+\sigma)$

$$
\begin{aligned}
|\cos w+1| & =\left|\int_{0}^{1}(1-t) \frac{d^{2}}{d t^{2}} \cos (\pi+t(w-\pi))\right|=\frac{1}{2}|\varrho|^{2} \sup _{\left|\varrho^{\prime}\right| \leq \varrho}\left|\cos \left(\pi+\varrho^{\prime}\right)\right| \\
& \leq \frac{1}{2}|\varrho|^{2} e^{\left|\varrho_{2}\right|} \leq \frac{1}{2}|\varrho|^{2}(1+\sigma)
\end{aligned}
$$

and using again the second inequality in (A.127), then inequality in (A.128) follows. The inequality in (A.129) is obtained via the second inequality in (A.127) and

$$
\begin{aligned}
\left|\sqrt{\chi_{j}^{2}-\Theta_{j}^{2}}-\sqrt{\chi_{j-1}^{2}-\Theta_{j}^{2}}\right| & =\frac{\left|\chi_{j-1}^{2}-\chi_{j}^{2}\right|}{\left|\sqrt{\chi_{j}^{2}-\Theta_{j}^{2}}+\sqrt{\chi_{j-1}^{2}-\Theta_{j}^{2}}\right|} \\
& \geq \frac{\left.|| \chi_{j-1}\right|^{2}-\left|\chi_{j}\right|^{2} \mid}{\left|\sqrt{\chi_{j}^{2}-\Theta_{j}^{2}}+\sqrt{\chi_{j-1}^{2}-\Theta_{j}^{2}}\right|} \\
& \geq \frac{|| \chi_{j-1}|-| \chi_{j}||}{\sqrt{1+\sigma^{2}}} .
\end{aligned}
$$

Lemma A.3. If $A, B \in \mathbb{C}$ and $a, \Delta \in \mathbb{R}_{+}$verify $|A-B| \geq \Delta$ and $\operatorname{Re} \bar{B}(A-B) \geq$ $a|B| \Delta$, where $0<a<1$, then ||$A|-| B||>a \Delta$.

Proof. Let $D:=A-B$. Then ||$A|-| B||=|| B+D|-| B|| \leq a \Delta$ implies

$$
|B|^{2}+|D|^{2}+2 \operatorname{Re} \bar{B} D=|B+D|^{2} \leq(|B|+a \Delta)^{2}=|B|^{2}+a^{2}(\Delta)^{2}+2 a|B| \Delta .
$$

This contradicts assumptions $|D| \geq \Delta>a \Delta$ and $\operatorname{Re} \bar{B} D \geq a|B| \Delta$. 
This is a free offprint provided to the author by the publisher. Copyright restrictions may apply. 


\section{APPENDIX B}

\section{Proof of Lemma 3.2}

In this chapter, we prove the formulae (3.40) and (3.41) given in Lemma 3.2 , We recall the following result

Proposition B.1 $([\mathbf{2 8}])$. Let $\mathfrak{X}=\mathfrak{X}_{1} \times \cdots \times \mathfrak{X}_{n} \subset \mathbb{R}^{5} \times \cdots \times \mathbb{R}^{5}$ and let

$$
\left(\ell_{k}, \mathrm{X}_{k}\right) \in \mathbb{T}^{1} \times \mathfrak{X}_{k} \rightarrow\left(y_{\phi}^{(k)}\left(\ell_{k}, \mathrm{X}_{k}\right), x_{\phi}^{(k)}\left(\ell_{k}, \mathrm{X}_{k}\right)\right) \in \mathbb{R}^{3} \times \mathbb{R}^{3} \quad k=1, \cdots, n
$$

be mappings such that, for $1 \leq i<j \leq n$

(A) the map

$$
\phi_{i j}: \quad\left(\ell_{i}, \ell_{j}, \mathrm{X}_{i}, \mathrm{X}_{j}\right) \rightarrow\left(y_{\phi}^{(i)}, y_{\phi}^{(2)}, x_{\phi}^{(j)}, x_{\phi}^{(2)}\right)
$$

is symplectomorphism of $\mathbb{T}^{2} \times \mathfrak{X}_{i} \times \mathfrak{X}_{j}$ into $\mathbb{R}^{12}$.

(B) The map $\left(\ell_{j}, \mathrm{X}_{j}\right) \rightarrow\left(y_{\phi}^{(2)}\left(\ell_{j}, \mathrm{X}_{j}\right), x_{\phi}^{(2)}\left(\ell_{j}, \mathrm{X}_{j}\right)\right)$ verifies

$$
\frac{\left\|y_{\phi}^{(2)}\left(\ell_{j}, \mathrm{X}_{j}\right)\right\|^{2}}{2 m_{j}}-\frac{\mathfrak{m}_{j} \mathfrak{M}_{j}}{\left\|x_{\phi}^{(2)}\left(\ell_{j}, \mathrm{X}_{j}\right)\right\|}=-\frac{\mathfrak{m}_{j}^{3} \mathfrak{M}_{j}^{2}}{2 \Lambda_{j}^{2}}
$$

where $\Lambda_{j}$ is the variable conjugated to $\ell_{j}$ in this symplectomorphism.

Then the function

$$
\begin{aligned}
\mathrm{P}^{(i)}\left(\ell_{i}, \mathrm{X}\right):=- & \frac{1}{2 \pi} \int_{\mathbb{T}} d \ell_{j} \\
& \frac{3\left(x_{\phi}^{(i)}\left(\ell_{i}, \mathrm{X}_{i}\right) \cdot x_{\phi}^{(j)}\left(\ell_{j}, \mathrm{X}_{j}\right)\right)^{2}-\left\|x_{\phi}^{(i)}\left(\ell_{i}, \mathrm{X}_{i}\right)\right\|^{2}\left\|x_{\phi}^{(j)}\left(\ell_{j}, \mathrm{X}_{j}\right)\right\|^{2}}{2\left\|x_{\phi}^{(j)}\left(\ell_{j}, \mathrm{X}_{j}\right)\right\|^{5}}
\end{aligned}
$$

is given by

$$
\mathrm{P}^{(i)}=\frac{\mathfrak{M}_{j} \mathfrak{m}_{j}^{2}}{4} \frac{3\left(x_{\phi}^{(i)} \cdot \mathrm{C}_{\phi}^{(j)}\right)^{2}-\left\|x_{\phi}^{(i)}\right\|^{2}\left\|\mathrm{C}_{\phi}^{(j)}\right\|^{2}}{\left\|\mathrm{C}_{\phi}^{(j)}\right\|^{4}} \frac{1}{2 \pi} \int_{\mathbb{T}} \frac{d \ell_{j}}{\left\|x_{\phi}^{(j)}\right\|^{2}} .
$$

with $\mathrm{C}_{\phi}^{(j)}(\mathrm{X}):=x_{\phi}^{(j)}\left(\ell_{j}, \mathrm{X}\right) \times y_{\phi}^{(j)}\left(\ell_{j}, \mathrm{X}\right)$.

Even though the $(i, j)$ projections of the $\mathcal{P}$-map do not verify assumption $(\mathrm{A})$, one has

Corollary B.1. The formula (B.130) applies also to the $\mathcal{P}$-map, or, more in general, to any Kepler map $\mathcal{K}$ related to the map Del in Definition 2.5 via

$$
\mathrm{X}_{\mathcal{D} e \ell}=\mathfrak{F}(\mathrm{X}) .
$$

Proof. Del verifies (A) and (B). 
In particular, we have an expression for the second-order term of the doubly averaged Newtonian potential

$$
\begin{aligned}
{\overline{f_{\mathcal{K}}^{i j}}}^{(2)}:= & -\frac{m_{i} m_{j}}{(2 \pi)^{2}} \int_{\mathbb{T}^{2}} d \ell_{i} d \ell_{j} \\
& \frac{3\left(x_{\mathcal{K}}^{(i)}\left(\ell_{i}, \mathrm{X}_{\mathcal{K}}\right) \cdot x_{\mathcal{K}}^{(j)}\left(\ell_{j}, \mathrm{X}_{\mathcal{K}}\right)\right)^{2}-\left\|x_{\mathcal{K}}^{(i)}\left(\ell_{i}, \mathrm{X}_{\mathcal{K}}\right)\right\|^{2}\left\|x_{\mathcal{K}}^{(j)}\left(\ell_{j}, \mathrm{X}_{\mathcal{K}}\right)\right\|^{2}}{2\left\|x_{\mathcal{K}}^{(j)}\left(\ell_{j}, \mathrm{X}_{\mathcal{K}}\right)\right\|^{5}}
\end{aligned}
$$

Corollary B.2. For any $\mathcal{K}$ as in Corollary B.1,

$$
\begin{aligned}
{\overline{f_{\mathcal{K}}^{i j}}}^{(2)} & =m_{i} m_{j} \frac{a_{i}^{2}}{4 a_{j}^{3}} \frac{\Lambda_{j}^{3}}{\left\|\mathrm{C}_{\mathcal{K}}^{(j)}\right\|^{5}}\left[-\left(\frac{5}{2}-\frac{3}{2} \frac{\left\|\mathrm{C}_{\mathcal{K}}^{(i)}\right\|^{2}}{\Lambda_{i}^{2}}\right)\left\|\mathrm{C}_{\mathcal{K}}^{(j)}\right\|^{2}\right. \\
& \left.+\frac{3}{2}\left(5-4 \frac{\left\|\mathrm{C}_{\mathcal{K}}^{(i)}\right\|^{2}}{\Lambda_{i}^{2}}\right)\left(P_{\mathcal{K}}^{(i)} \cdot \mathrm{C}_{\mathcal{K}}^{(j)}\right)^{2}+\frac{3}{2} \frac{\left\|\mathrm{C}_{\mathcal{K}}^{(i)}\right\|^{2}}{\Lambda_{i}^{2}}\left(Q_{\mathcal{K}}^{(i)} \cdot \mathrm{C}_{\mathcal{K}}^{(j)}\right)^{2}\right]
\end{aligned}
$$

Proof. Lemma B.1 implies that

$$
\begin{aligned}
\bar{f}_{\mathcal{K}}^{i j}(2)= & m_{i} m_{j} \frac{M_{j} m_{j}^{2}}{4} \frac{\frac{1}{2 \pi} \int_{\mathbb{T}}\left(3\left(x_{\mathcal{K}}^{(i)} \cdot \mathrm{C}_{\mathcal{K}}^{(j)}\right)^{2}-\left\|x_{\mathcal{K}}^{(i)}\right\|^{2}\left\|\mathrm{C}_{\mathcal{K}}^{(j)}\right\|^{2}\right) d \ell_{i}}{\left\|\mathrm{C}_{\mathcal{K}}^{(j)}\right\|^{4}} \\
& \times \frac{1}{2 \pi} \int_{\mathbb{T}} \frac{d \ell_{j}}{\left\|x_{\mathcal{K}}^{(j)}\right\|^{2}} .
\end{aligned}
$$

By (2.1)

$$
\begin{aligned}
x_{\mathcal{K}}^{(i)} \cdot \mathrm{C}_{\mathcal{K}}^{(j)} & =\left(\mathrm{a}_{i, \mathcal{K}} P_{\mathcal{K}}^{(i)}+\mathrm{b}_{i, \mathcal{K}} Q_{\mathcal{K}}^{(i)}\right) \cdot \mathrm{C}_{\mathcal{K}}^{(j)} \\
& =\mathrm{a}_{i, \mathcal{K}} P_{\mathcal{K}}^{(i)} \cdot \mathrm{C}_{\mathcal{K}}^{(j)}+\mathrm{b}_{i, \mathcal{K}} Q_{\mathcal{K}}^{(i)} \cdot \mathrm{C}_{\mathcal{K}}^{(j)}
\end{aligned}
$$

Therefore, squaring, $\ell_{i}$-averaging and using

$$
\begin{aligned}
\frac{1}{2 \pi} \int_{\mathbb{T}}\left(\mathrm{a}_{i, \mathcal{K}}\right)^{2} d \ell_{i} & =\frac{a_{i}^{2}}{2}\left(5-4 \frac{\left\|\mathrm{C}_{\mathcal{K}}^{(i)}\right\|^{2}}{\Lambda_{i}^{2}}\right) \\
\frac{1}{2 \pi} \int_{\mathbb{T}}\left(\mathrm{b}_{i, \mathcal{K}}\right)^{2} d \ell_{i} & =\frac{a_{i}^{2}}{2} \frac{\left\|\mathrm{C}_{\mathcal{K}}^{(i)}\right\|^{2}}{\Lambda_{i}^{2}} \\
\frac{1}{2 \pi} \int_{\mathbb{T}} \mathrm{a}_{i, \mathcal{K}} \mathrm{b}_{i, \mathcal{K}} d \ell_{i} & =0
\end{aligned}
$$

we obtain

$$
\begin{aligned}
\frac{1}{2 \pi} \int_{\mathbb{T}}\left(x_{\mathcal{K}}^{(i)} \cdot \mathrm{C}_{\mathcal{K}}^{(j)}\right)^{2} d \ell_{i} & =\frac{a_{i}^{2}}{2}\left(5-4 \frac{\left\|\mathrm{C}_{\mathcal{K}}^{(i)}\right\|^{2}}{\Lambda_{i}^{2}}\right)\left(P_{\mathcal{K}}^{(i)} \cdot \mathrm{C}_{\mathcal{K}}^{(j)}\right)^{2} \\
& +\frac{a_{i}^{2}}{2} \frac{\left\|\mathrm{C}_{\mathcal{K}}^{(i)}\right\|^{2}}{\Lambda_{i}^{2}}\left(Q_{\mathcal{K}}^{(i)} \cdot \mathrm{C}_{\mathcal{K}}^{(j)}\right)^{2}
\end{aligned}
$$

Using finally

$$
\frac{1}{2 \pi} \int_{\mathbb{T}}\left\|x_{\mathcal{K}}^{(i)}\right\|^{2} d \ell_{i}=a_{i}^{2}\left(\frac{5}{2}-\frac{3}{2} \frac{\left\|\mathrm{C}_{\mathcal{K}}^{(i)}\right\|^{2}}{\Lambda_{i}^{2}}\right), \quad \frac{1}{2 \pi} \int_{\mathbb{T}} \frac{d \ell_{j}}{\left\|x_{\mathcal{K}}^{(j)}\right\|^{2}}=\frac{1}{a_{j}^{2}} \frac{\Lambda_{j}}{\left\|\mathrm{C}_{\mathcal{K}}^{(j)}\right\|}
$$

we obtain (B.131). 
Now we may proceed with proving the formulae in (3.40) and (3.41).

Proof OF OF (3.40). We apply Corollary B.2 with $\mathcal{K}=\mathcal{P}, i=n-1, j=n$. Using $\left\|\mathrm{C}_{\mathcal{P}}^{(n)}\right\|=\chi_{n-1}($ see (2.17) $), \mathrm{C}_{\mathcal{P}}^{(n)}=\mathrm{S}_{\mathcal{P}}^{(n)}$ and Eq. (2.3), Proposition 2.1, and Remark 2.2, we have

$$
\begin{aligned}
P_{\mathcal{P}}^{(n-1)} \cdot \mathrm{S}_{\mathcal{P}}^{(n)} & =\Theta_{n-1} \\
Q_{\mathcal{P}}^{(n-1)} \cdot \mathrm{S}_{\mathcal{P}}^{(n)} & =\frac{1}{\left\|\mathrm{C}_{\mathcal{P}}^{(n-1)}\right\|}\left(\left(\mathrm{S}_{\mathcal{P}}^{(n-1)}-\mathrm{S}_{\mathcal{P}}^{(n)}\right) \times P_{\mathcal{P}}^{(n-1)}\right) \cdot \mathrm{S}_{\mathcal{P}}^{(n)} \\
& =\frac{1}{\left\|\mathrm{C}_{\mathcal{P}}^{(n-1)}\right\|} \mathrm{S}_{\mathcal{P}}^{(n-1)} \times \mathrm{P}_{\mathcal{P}}^{(n-1)} \cdot \mathrm{S}_{\mathcal{P}}^{(n)} \\
& =\frac{1}{\left\|\mathrm{C}_{\mathcal{P}}^{(n-1)}\right\|} \sqrt{\left(\chi_{n-1}^{2}-\Theta_{n-1}^{2}\right)\left(\chi_{n-2}^{2}-\Theta_{n-1}^{2}\right)} \sin \vartheta_{n-1} .
\end{aligned}
$$

Proof of (3.41). By Corollary B.2 with $\mathcal{K}=\mathcal{P}, j=i+1$, we find, for ${\overline{f_{\mathcal{P}}^{i, i+1}}}^{(2)}$ an expression as in (B.131), replacing $(n-1, n)$ with $(i, i+1)$.

$$
\begin{aligned}
& P_{\mathcal{P}}^{(i)} \cdot \mathrm{C}_{\mathcal{P}}^{(i+1)}=P_{\mathcal{P}}^{(i)} \cdot\left(\mathrm{S}_{\mathcal{P}}^{(i+1)}-\mathrm{S}_{\mathcal{P}}^{(i+2)}\right)=\Theta_{i}-P_{\mathcal{P}}^{(i)} \cdot \mathrm{S}_{\mathcal{P}}^{(i+2)} \\
& Q_{\mathcal{P}}^{(i)} \cdot \mathrm{C}_{\mathcal{P}}^{(i+1)}=Q_{\mathcal{P}}^{(i)} \cdot\left(\mathrm{S}_{\mathcal{P}}^{(i+1)}-\mathrm{S}_{\mathcal{P}}^{(i+2)}\right)=\frac{1}{\left\|\mathrm{C}_{\mathcal{P}}^{(i)}\right\|}\left(\sqrt{\left(\chi_{i}^{2}-\Theta_{i}^{2}\right)\left(\chi_{i-1}^{2}-\Theta_{i}^{2}\right)} \sin \vartheta_{i}\right. \\
& \left.-\mathrm{S}_{\mathcal{P}}^{(i)} \times \mathrm{P}_{\mathcal{P}}^{(i)} \cdot \mathrm{S}_{\mathcal{P}}^{(i+2)}-\mathrm{P}_{\mathcal{P}}^{(i)} \times \mathrm{S}_{\mathcal{P}}^{(i+1)} \cdot \mathrm{S}_{\mathcal{P}}^{(i+2)}\right) .
\end{aligned}
$$

Now, when $\left(\Theta_{i+1}, \vartheta_{i+1}\right)=(0, \pi),\left\|\mathrm{C}_{\mathcal{P}}^{(i+1)}\right\|$ reduces to

$$
\left\|\mathrm{C}_{\mathcal{P}}^{(i+1)}\right\|=\chi_{i}-\chi_{i+1}
$$

(provided $\left.\arg \left(\chi_{i}-\chi_{i+1}\right) \in\left(-\frac{\pi}{2}, \frac{\pi}{2}\right] \bmod 2 \pi\right)$ and $\mathrm{S}_{\mathcal{P}}^{(i+2)} \| \mathrm{S}_{\mathcal{P}}^{(i+1)}$, so

$$
\mathrm{S}_{\mathcal{P}}^{(i+2)}=\frac{\chi_{i+1}}{\chi_{i}} \mathrm{~S}_{\mathcal{P}}^{(i+1)}
$$

and hence, the extra-terms in (B.132) reduce to

$$
\begin{aligned}
& P_{\mathcal{P}}^{i)} \cdot \mathrm{S}_{\mathcal{P}}^{(i+2)}=\Theta_{i} \frac{\chi_{i+1}}{\chi_{i}} \\
& \mathrm{~S}_{\mathcal{P}}^{(i)} \times \mathrm{P}_{\mathcal{P}}^{(i)} \cdot \mathrm{S}_{\mathcal{P}}^{(i+2)}=\frac{\chi_{i+1}}{\chi_{i}} \sqrt{\chi_{i-1}^{2}-\Theta_{i}^{2}} \sqrt{\chi_{i}^{2}-\Theta_{i}^{2}} \sin \vartheta_{i} \\
& \mathrm{P}_{\mathcal{P}}^{(i)} \times \mathrm{S}_{\mathcal{P}}^{(i+1)} \cdot \mathrm{S}_{\mathcal{P}}^{(i+2)}=0 .
\end{aligned}
$$

Then (3.41) readily follows. 
This is a free offprint provided to the author by the publisher. Copyright restrictions may apply. 


\section{APPENDIX C}

\section{Checking the non-degeneracy condition}

In this chapter we prove statement 4 of Proposition 5.2 .

Due to the form of $\mathrm{h}_{\mathfrak{s e c}}$ in (5.73) $-\left(\underline{5.74)}\right.$ ) and to the bound for $\widetilde{\mathrm{h}_{\mathfrak{s e c}, h}^{i}}$ in (․76) , it is sufficient to prove that the maps

$$
\zeta_{i}^{(h)} \rightarrow \overline{\omega_{\mathfrak{s e c}}^{i}}:=\partial_{\zeta_{i}^{(h)}} \overline{\mathrm{h}_{\mathfrak{s e c}}^{i}}\left(\zeta_{i}^{(h)}, \Lambda_{n-h}^{(h)}, \Lambda_{n-h+1}^{(h)}\right)
$$

in (5.74), where

$$
\zeta_{i}^{(h)}=\left\{\begin{array}{lll}
\left(\frac{\left(p_{1}^{(h)}\right)^{2}+\left(q_{1}^{(h)}\right)^{2}}{2}, \chi_{1}^{(h)}\right) & i=1 & \& n=2 \\
\left(\frac{\left(p_{n-1}^{(h)}\right)^{2}+\left(q_{n-1}^{(h)}\right)^{2}}{2}, \chi_{n-2}^{(h)}, \chi_{n-1}^{(h)}\right) & i=n-1 & \& n \geq 3 \\
\left(\frac{\left(p_{i}^{(h)}\right)^{2}+\left(q_{i}^{(h)}\right)^{2}}{2}, \chi_{i-1}^{(h)}\right) & i=2, \cdots, n-2 & \& n \geq 4 \\
\frac{\left(p_{1}^{(h)}\right)^{2}+\left(q_{1}^{(h)}\right)^{2}}{2} & i=1 & \& n \geq 3
\end{array}\right.
$$

are diffeomorphisms, with non-vanishing Hessian matrices. We shall do this verifications for just one of the cases above, and we choose the second case in the list, $i=n-1$, for $n \geq 3$. The explicit expression of $\overline{h_{\mathfrak{s e c}}^{n-1}}$ is given in (5.98)-(5.99). We neglect the coefficient $\mathcal{A}_{n-1}$ (which does not depend on $\zeta_{n-1}^{(h)}$ ) and we denote

$$
\left.\widehat{\mathrm{h}_{\mathfrak{s e c}}^{n-1}}=\mathrm{E}_{n-1}+\Omega_{n-1} \frac{p_{n-1}^{2}+q_{n-1}^{2}}{2}+\tau_{n-1}\left(\frac{p_{n-1}^{2}+q_{n-1}^{2}}{2}\right)^{2}+\mathrm{O}\left(p_{n-1}, q_{n-1}\right)^{6}\right]
$$

the function $\overline{\mathrm{h}_{\mathfrak{s e c}}^{n-1}}$ thus rescaled, and $\widehat{\omega_{\mathfrak{i n t}}^{n-1}}$ its gradient with respect to

$$
\left(\frac{\left(p_{n-1}^{(h)}\right)^{2}+\left(q_{n-1}^{(h)}\right)^{2}}{2}, \chi_{n-2}, \chi_{n-1}\right) .
$$

A perturbative argument shows that, under the choices of Corollary 4.1 the frequency-map with respect to $\left(\chi_{n-2}, \chi_{n-1}\right)$ associated to

$$
\mathrm{E}_{n-1}=-\frac{\Lambda_{n}^{3}}{2 \chi_{n-1}^{3}}\left(5-3 \frac{\left(\chi_{n-2}-\chi_{n-1}\right)^{2}}{\Lambda_{n-1}^{2}}\right)
$$

is an injection of its domain and hence, by another perturbative argument, so is the gradient of $\widehat{\mathrm{h}_{\mathfrak{s e c}}^{n-1}}$ with respect to the same coordinates, for any fixed value of $\frac{p_{n-1}^{2}+q_{n-1}^{2}}{2}$. On the other hand, since $\tau_{n-1}$ does not vanish under the same 
assumptions of Corollary 4.1, $\widehat{\omega_{\mathfrak{i n t}}^{n-1}}$ is an injection. The computation shows that the Jacobian of $\widehat{\omega_{\mathfrak{i n t}}^{n-1}}$ does not vanish. 


\section{APPENDIX D}

\section{Some results from perturbation theory}

\section{D.1. A multi-scale normal form theorem}

The purpose of this chapter is to present a normal form result which takes into account different scale lengths. It is a particularization of [31, Normal Form Lemma, p. 192] and uses the same techniques of that paper.

Following [31, the notations are as follows.

- If $A \subset \mathbb{R}^{\nu}$ is open and connected, $\mathbb{T}:=\mathbb{R} /(2 \pi \mathbb{Z})$ is the usual flat torus, $r, s$ are positive numbers, we denote as $A_{r}:=\bigcup_{x \in A}\left\{z \in \mathbb{C}^{\nu}: \quad z \in\right.$ $\left.B_{r}^{\nu}(x)\right\}$ the complex $r$-neighborhood of $A . \mathbb{T}_{s}^{\nu}$ will denote the complex set $\mathbb{T}+\mathrm{i}[-s, s]$. As usual, $B_{r}^{\nu}(x)$ denotes the ball in $\mathbb{C}^{\nu}$ with radius $r$ centered at $x$, accordingly to a prefixed norm $|\cdot|$ of $\mathbb{C}^{\nu}$.

- If $f=f(u, p, q, \varphi)$ is real-analytic for $(u, p, q, \varphi) \in W_{v, s, \varepsilon}=U_{v} \times B_{\varepsilon}^{2 \ell} \times \mathbb{T}_{s}^{\nu}$, and affords the Taylor-Fourier expansion

$$
f=\sum_{k \in \mathbb{Z}^{m}} f_{k, \alpha, \beta}(u) e^{i k \cdot \varphi} \prod_{j=1}^{\ell}\left(\frac{p_{j}-\mathrm{i} q_{j}}{\sqrt{2}}\right)^{\alpha_{j}}\left(\frac{p_{j}+\mathrm{i} q_{j}}{\mathrm{i} \sqrt{2}}\right)^{\beta_{j}},
$$

we denote as $\|f\|_{v, s, \varepsilon}$ its "sup-(Taylor, Fourier) norm":

$$
\|f\|_{v, s, \varepsilon}:=\sum_{\substack{(a, b) \in \mathbb{N}^{2 \ell} \\ k \in \mathbb{Z}^{\nu}}} \sup _{u \in U_{v}}\left|f_{\alpha, \beta, k}(u)\right| e^{|k| s} \varepsilon^{|(\alpha, \beta)|}
$$

with $|k|:=|k|_{1},|(\alpha, \beta)|:=|\alpha|_{1}+|\beta|_{1}$.

- If $f$ is as in the previous item, $K>0$ and $\mathfrak{L}=\mathfrak{L}_{1} \times \mathfrak{L}_{2}$ is a sub-lattice of $\mathbb{Z}^{\nu} \times \mathbb{Z}^{\ell}, T_{K} f$ and $\Pi_{\mathfrak{L}} f$ denote, respectively, the $K$-truncation and the $\mathfrak{L}$-projection of $f$ :

$$
\begin{aligned}
& T_{K} f:=\sum_{|(\alpha, \beta)| \leq K,|k| \leq K} f_{\alpha, \beta, k}(u) e^{i k \cdot \varphi} \prod_{j=1}^{\ell}\left(\frac{p_{j}-\mathrm{i} q_{j}}{\sqrt{2}}\right)^{\alpha_{j}}\left(\frac{p_{j}+\mathrm{i} q_{j}}{\mathrm{i} \sqrt{2}}\right)^{\beta_{j}} \\
& \Pi_{\mathcal{L} f}:=\sum_{\substack{k \in \mathfrak{L}_{1} \\
\alpha-\beta \in \mathfrak{L}_{2}}} f_{\alpha, \beta, k}(u) e^{i k \cdot \varphi} \prod_{j=1}^{\ell}\left(\frac{p_{j}-\mathrm{i} q_{j}}{\sqrt{2}}\right)^{\alpha_{j}}\left(\frac{p_{j}+\mathrm{i} q_{j}}{\mathrm{i} \sqrt{2}}\right)^{\beta_{j}} .
\end{aligned}
$$

Proposition D.1 (Multi-scale normal form). Let

$$
\nu, \quad \ell, \quad 1 \leq m_{1}<\cdots<m_{N}=m
$$

be natural numbers;

$A \subset \mathbb{R}^{\nu}, B \subset \mathbb{R}^{2 \ell}, C_{1}, C_{1}^{\prime} \subset \mathbb{R}^{m_{1}}, C_{2}, C_{2}^{\prime} \subset \mathbb{R}^{m_{2}-m_{1}}, \cdots, C_{N}, C_{N}^{\prime} \subset \mathbb{R}^{m_{N}-m_{N-1}}$, 
be open and connected sets;

$$
r, s, \varepsilon, \rho_{1} \geq \rho_{2} \cdots \geq \rho_{N}, \rho_{1}^{\prime} \geq \rho_{2}^{\prime} \cdots \geq \rho_{N}^{\prime}
$$

positive numbers. Put

$$
\begin{array}{ll}
v_{i}:=\left(r, \rho_{1}, \cdots, \rho_{i}, \rho_{1}^{\prime}, \cdots, \rho_{i}^{\prime}\right), & v:=v_{N} \\
U_{v_{i}}^{(i)}:=A_{r} \times C_{1 \rho_{1}} \times \cdots \times C_{i \rho_{i}} \times C_{1 \rho_{1}^{\prime}}^{\prime} \times \cdots \times C_{i \rho_{i}^{\prime}}^{\prime}, & U_{v}:=U_{v_{N}}^{(N)} \\
W_{v_{i}, s, \varepsilon}^{(i)}:=U_{v_{i}}^{(i)} \times \mathbb{T}_{s}^{\nu} \times B_{\varepsilon}, & W_{v, s, \varepsilon}:=W_{v_{N}, s, \varepsilon}^{(N)},
\end{array}
$$

with $i=1, \cdots, N$.

Let $\mathfrak{a}, K>0$ with $0<s<6 \log 6 / 5$ and $K s \geq 12$; let also $\mathfrak{L}$ and $\mathfrak{Z}_{1}, \cdots, \mathfrak{Z}_{N}$ be sub-lattices of $\mathbb{Z}^{\ell} \times \mathbb{Z}^{\nu}$ and let $\mathfrak{Z}:=\mathfrak{Z}_{1} \cup \cdots \cup \mathfrak{Z}_{N}$.

Let

$$
H(u, \varphi, p, q)=\mathrm{h}(p, q, I)+f(u, \varphi, p, q)
$$

be real-analytic for $(u, \varphi, p, q) \in W_{v, s, \varepsilon}$, where $u:=(I, \eta, \xi)=\left(I_{1}, \cdots, I_{\nu}, \eta_{1}\right.$, $\left.\cdots, \eta_{m}, \xi_{1}, \cdots, \xi_{m}\right)$. Suppose that

(i) $h$ depends on $(p, q)$ only via $\frac{p_{i}^{2}+q_{i}^{2}}{2}$, with the frequency map $\omega=\left(\omega_{1}, \cdots\right.$, $\left.\omega_{\ell}, \omega_{\ell+1}, \cdots, \omega_{\ell+\nu}\right)$ defined via

$$
\omega_{i}:= \begin{cases}\frac{\partial_{p_{i}^{2}+q_{i}^{2}} \mathrm{~h}}{2} \quad 1 \leq i \leq \ell \\ \partial_{I_{i-\ell}} \mathrm{h} \quad \ell+1 \leq i \leq \ell+\nu\end{cases}
$$

verifying

$$
\left|\omega(p, q, I) \cdot\left(k^{\prime}, k\right)\right| \geq \mathfrak{a} \quad \forall\left(k^{\prime}, k\right) \in \mathfrak{Z} \backslash \mathfrak{L},\left|\left(k^{\prime}, k\right)\right| \leq K
$$

and all $(p, q, I) \in B_{\varepsilon}^{2 \ell} \times A_{r}$;

(ii) $f$ is a sum

$$
f=\sum_{i=1}^{N} f_{i}\left(u_{i}, \varphi, p, q\right)
$$

where $f_{i}$ is real-analytic on $W_{v_{i}, s, \varepsilon}^{(i)}$ and has the form

$$
f_{i}\left(u_{i}, \varphi, p, q\right)=\sum_{\left(\alpha^{-} \alpha^{+}, k\right) \in \mathfrak{Z}_{i}} f_{k, \alpha^{-}, \alpha^{+}}^{i}\left(u_{i}\right) \prod_{j=1}^{\nu} e^{\mathrm{i} k_{j} \varphi_{j}} \prod_{k=1}^{\ell}\left(\frac{p_{k}-\mathrm{i} q_{k}}{\sqrt{2}}\right)^{\alpha_{k}^{-}}\left(\frac{p_{k}+\mathrm{i} q_{k}}{\sqrt{2} \mathrm{i}}\right)^{\alpha_{k}^{+}}
$$

with

$$
u_{i}:=\left(I, \eta^{i}, \xi^{i}\right):=\left(I_{1}, \cdots, I_{\nu}, \eta_{1}, \cdots, \eta_{m_{i}}, \xi_{1}, \cdots, \xi_{m_{i}}\right) ;
$$

(iii) the following "smallness" conditions hold. If

$$
c_{i}:=e\left(1+\ell_{i} e+m_{i} e\right) / 2, \quad d_{i}:=\min \left\{r s, \varepsilon^{2}, \rho_{i} \rho_{i}^{\prime}\right\}
$$

with e denoting Neper number, then

$$
\left\|f_{i}\right\|_{W_{v_{i}, s, \varepsilon}^{(i)}} \leq E_{i}, \quad \sum_{i=1}^{N} \frac{7}{6}\left(\frac{9}{8}\right)^{i-1} \frac{2^{7} c_{i} K s}{\mathfrak{a} d_{i}} E_{i}<1 .
$$


Then, one can find a real-analytic and symplectic transformation

$$
\Phi: \quad W_{v / 6^{N}, s / 6^{N}, \varepsilon / 6^{N}} \rightarrow W_{v, \sigma, \varepsilon}
$$

which conjugates $H$ to

$$
H_{*}(u, \varphi, p, q):=H \circ \Phi=\mathrm{h}(I, p, q)+\sum_{i=1}^{N} g_{i}\left(u_{i}, \varphi, p, q\right)+\sum_{i=1}^{N} f_{i}^{*}(u, \varphi, p, q),
$$

where $g_{i}, f_{i}$ verify

$$
\begin{aligned}
g_{i} & =\Pi_{\mathfrak{Z}_{i} \cap \mathfrak{L} T_{K} g_{i}} \\
\left\|g_{i}-\Pi_{\mathfrak{Z}_{i} \cap \mathfrak{L}} T_{K} f_{i}\right\|_{v_{i} / 6^{N}, \sigma / 6^{N}, \varepsilon / 6^{N}} & \leq\left(\frac{9}{8}\right)^{2(i-1)} \frac{2^{7} c_{i}\left\|f_{i}\right\|_{v_{i}, s, \varepsilon}^{2}}{\mathfrak{a} d_{i}} \\
& +\frac{7}{6}\left(\frac{9}{8}\right)^{2(i-1)} \sum_{j=1}^{i-1} \frac{2^{7} c_{j}\left\|f_{j}\right\|_{v_{j}, s, \varepsilon}}{\mathfrak{a} d_{j}}\left\|f_{i}\right\|_{v_{i}, s, \varepsilon} \\
& +\sum_{k=1}^{i-1}\left(\frac{9}{8}\right)^{i-1-k} \frac{2^{4} c_{k}\left\|f_{k}\right\|_{v_{k}, s, \varepsilon} K s}{\mathfrak{a} d_{k}}\left\|f_{i}\right\|_{v_{i}, s, \varepsilon} \\
\left\|f_{i}^{*}\right\|_{v_{i} / 6^{N}, s / 6^{N}, \varepsilon / 6^{N}} & \leq\left(\frac{9}{8}\right)^{N-1} e^{-K s / 6^{i}}\left\|f_{i}\right\|_{v_{i}, s, \varepsilon}
\end{aligned}
$$

Finally, $\Phi$ is close to the identity in the following sense. Given $F$, real-analytic on $W_{v_{i} / 6^{N}, s / 6^{N}, \varepsilon / 6^{N}}^{(i)}$

$$
\|F \circ \Phi-F\|_{v / 6^{N}, s / 6^{N}, \varepsilon / 6^{N}} \leq \sum_{k=1}^{N}\left(\frac{9}{8}\right)^{N-k} \frac{2^{4} c_{k}\left\|f_{k}\right\|_{v_{k}, s, \varepsilon} K s}{\mathfrak{a} d_{k, i}}\|F\|_{v_{i} / 6^{N}, s / 6^{N}, \varepsilon / 6^{N}}
$$

with $d_{k, i}:=\max \left\{d_{k}, d_{i}\right\}$.

The proof of Proposition D.1 is based on the following

Lemma D.1. Let $\bar{N} \in \mathbb{N}, \nu, \ell, m_{i}, A, B, C_{i}, C_{i}^{\prime}, r, s, \rho_{i}, \rho_{i}^{\prime}, U_{v_{i}}^{(i)}, W_{v_{i}, s, \varepsilon}^{(i)}, c_{i}$, $d_{i}$, with $i=1, \cdots, \bar{N}+1$, be as in Proposition D.1;

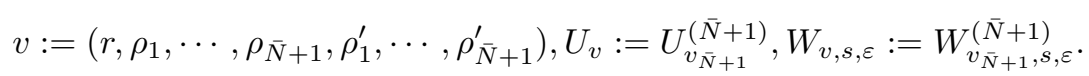

Let

$$
H(p, q, I, \varphi, \eta, \xi)=\mathrm{h}(p, q, I)+g(p, q, I, \varphi, \eta, \xi)+f(p, q, I, \varphi, \eta, \xi)
$$

be real-analytic for $(u, \varphi, p, q) \in W_{v, s, \varepsilon}$. Suppose assumption (i) of Proposition D.1 and, moreover, the following ones

(ii) $g$ is a sum

$$
g=\sum_{i=1}^{\bar{N}} g_{i}\left(u_{i}, \varphi, p, q\right)
$$

where $g_{i}$ is real-analytic on $W_{v_{i}, s, \varepsilon}^{(i)}$ and $u_{i}$ is as in (D.137);

(iii) $g_{1}, \cdots, g_{\bar{N}}$ and $f$ satisfy

$$
g_{i}=\Pi_{\mathfrak{L}} g_{i}, \quad f=\Pi_{\mathfrak{J}} f
$$


and

$$
\sum_{i=1}^{\bar{N}} \frac{2^{7} c_{i} K s}{\mathfrak{a} d_{i}}\left\|g_{i}\right\|_{v_{i}, s_{i}, \varepsilon_{i}}<1, \quad\|f\|_{v, s, \varepsilon}<\frac{\mathfrak{a} d_{\bar{N}+1}}{2^{7} c_{\bar{N}+1} K s} .
$$

Then, one can find a real-analytic and symplectic transformation

$$
\Phi: \quad\left(u^{\prime}, \varphi^{\prime}, p^{\prime}, q^{\prime}\right) \in W_{v / 6, s / 6, \varepsilon / 6} \rightarrow(u, \varphi, p, q) \in W_{v, \sigma, \varepsilon}
$$

such that

$$
H_{*}:=H \circ \Phi=h+g+g_{*}+f_{*},
$$

where $g_{*}=\Pi_{\mathfrak{Z} \cap \mathfrak{L}} T_{K} g_{*}$ is $\mathfrak{Z} \cap \mathfrak{L}$-resonant and the following bounds hold

$$
\begin{aligned}
\left\|g_{*}-T_{K} \Pi_{\mathfrak{Z} \cap \mathfrak{L}} f\right\|_{v / 6, \sigma / 6, \varepsilon / 6} & \leq\left(\frac{2^{7} c_{\bar{N}+1}\|f\|_{v, s, \varepsilon}}{\mathfrak{a} d_{\bar{N}+1}}+\sum_{i=1}^{n} \frac{2^{7} c_{i}\left\|g_{i}\right\|_{v_{i}, s, \varepsilon}}{\mathfrak{a} d_{i}}\right)\|f\|_{v, s, \varepsilon} \\
& \leq \frac{\|f\|_{v, s, \varepsilon}}{6} \\
\left\|f_{*}\right\|_{v / 6, \sigma / 6, \varepsilon / 6} & \leq e^{-K s / 6}\|f\|_{v, s, \varepsilon} .
\end{aligned}
$$

Finally, $\Phi$ is close to the identity in the following sense: for any $F$ which is realanalytic on $W_{v, s, \varepsilon}^{(i)}$,

$$
\|F \circ \Phi-\Phi\|_{v / 6, s / 6, \varepsilon / 6} \leq \frac{2^{4} c_{\bar{N}+1}\|f\|_{v, s, \varepsilon} K s}{\mathfrak{a} d_{i}}\|F\|_{v_{i}, s, \varepsilon}<\frac{1}{8}\|F\|_{v, s, \varepsilon} .
$$

The following Lemma is a trivial extension 1 of [31, Iterative Lemma]. Its proof is omitted.

Lemma D.2. Let $s=\left(s_{1}, \cdots, s_{\nu}\right), r=\left(r_{1}, \cdots, r_{\nu}\right), \varepsilon=\left(\varepsilon_{1}, \cdots, \varepsilon_{\ell}\right), \rho=$ $\left(\rho_{1}, \cdots, \rho_{m}\right), \rho^{\prime}=\left(\rho_{1}^{\prime}, \cdots, \rho_{m}^{\prime}\right), v:=\left(r, \rho, \rho^{\prime}\right), \hat{v}:=\left(\hat{r}, \hat{\rho}, \hat{\rho}^{\prime}\right)<v / 2, \hat{s}<s / 2$, $\hat{\varepsilon}<\varepsilon / 2$,

$$
\delta:=\min _{\substack{i=1, \cdots, \nu \\ j=1, \cdots, \ell \\ k=1, \cdots, m}}\left\{\hat{r}_{i} \hat{s}_{i}, \hat{\varepsilon}_{j}^{2}, \hat{\rho}_{k} \hat{\rho}_{k}^{\prime}\right\}
$$

Let

$H(u, \varphi, p, q)=\mathrm{h}(I, p, q)+g(u, \varphi, p, q)+f(u, \varphi, p, q) \quad g(u, \varphi, p, q)=\sum_{i=1}^{m} g_{i}(u, \varphi, p, q)$

be real-analytic on $W_{v, s, \varepsilon}$. Assume that inequality (D.134) and

$$
\|f\|_{v, s, \varepsilon}<\frac{\mathfrak{a} \delta}{c}
$$

are satisfied. Then one can find a real-analytic and symplectic transformation

$$
\Phi: W_{v-2 \hat{v}, s-2 \hat{s}, \varepsilon-2 \hat{\varepsilon}} \rightarrow W_{v, s, \varepsilon}
$$

\footnotetext{
${ }^{1}$ In order to obtain the extension it is sufficient to replace $\phi$ of [31, Appendix A] with
}

$$
\phi=\sum_{\substack{(\alpha-\beta, k) \in \mathfrak{K} \backslash \mathfrak{L} \\|(\alpha, \beta)| \leq K,|k| \leq K}} \frac{f_{k, \alpha, \beta}(u)}{\mathrm{i}(\alpha-\beta, k) \cdot \omega} e^{i k \cdot \varphi} \prod_{j=1}^{\ell}\left(\frac{p_{j}-\mathrm{i} q_{j}}{\sqrt{2}}\right)^{\alpha_{j}}\left(\frac{p_{j}+\mathrm{i} q_{j}}{\mathrm{i} \sqrt{2}}\right)^{\beta_{j}}
$$


defined by the time-one flou $X_{\phi}^{1} f:=f \circ \Phi$ of a suitable $\phi$ verifying

$$
\|\phi\|_{v, s, \varepsilon} \leq \frac{\|f\|_{v, s, \varepsilon}}{\mathfrak{a}}
$$

such that

$$
H_{+}:=H \circ \Phi=h+g+\Pi_{\mathfrak{L} \cap \mathfrak{Z}} f+f_{+}
$$

and, moreover, the following bounds hold

$$
\begin{aligned}
\left\|f_{+}\right\|_{v-2 \hat{v}, s-2 \hat{s}, \varepsilon-2 \hat{\varepsilon}} \leq & \left(1-\frac{c}{\mathfrak{a} \delta}\|f\|_{v, s, \varepsilon}\right)^{-1}\left[\frac{c}{\mathfrak{a} \delta}\|f\|_{v, s, \varepsilon}^{2}\right. \\
& \left.+e^{-K \hat{s}}\|f\|_{v, s, \varepsilon}+\left(\frac{\varepsilon-\hat{\varepsilon}}{\varepsilon}\right)^{K}\|f\|_{v, s, \varepsilon}+\|\{\phi, g\}\|_{v-\hat{v}, s-\hat{s}, \varepsilon-\hat{\varepsilon}}\right]
\end{aligned}
$$

Finally, for any real-analytic function $F$ on $W_{v, s, \varepsilon}$,

$$
\|F \circ \Phi-F\|_{v-2 \hat{v}, s-2 \hat{s}, \varepsilon-2 \hat{\varepsilon}} \leq \frac{\|\{\phi, F\}\|_{v-\hat{v}, s-\hat{s}, \varepsilon-\hat{\varepsilon}}}{1-\frac{c\|f\|_{v, s, \varepsilon}}{\mathfrak{a} \delta}}
$$

Proof of Lemma D.1. Following [31, the proof is obtained via iterate applications of Lemma D.2.

To avoid too many indices, we shall prove this lemma taking, in (D.141), $\bar{N}=1$; the extension to $\bar{N} \geq 1$ being straightforward. Namely, we take

$$
\begin{aligned}
& \rho_{1}=\cdots=\rho_{m_{1}}=\bar{\rho}, \quad \rho_{1}^{\prime}=\cdots=\rho_{m_{1}}^{\prime}=\bar{\rho}^{\prime} \\
& \rho_{m_{1}+1}=\cdots=\rho_{m}=\rho, \quad \rho_{m_{1}+1}^{\prime}=\cdots=\rho_{m}^{\prime}=\rho
\end{aligned}
$$

where $1 \leq m_{1}<m$. Letting

$$
\begin{aligned}
& v:=\left(r, \rho, \rho^{\prime}\right), \quad \bar{v}:=\left(r, \bar{\rho}, \bar{\rho}^{\prime}\right), \quad E:=\|f\|_{v, s, \varepsilon}, \quad G:=\|g\|_{\bar{v}, s, \varepsilon}, \\
& \bar{c}=c_{1}, \quad c=c_{2}, \bar{d}:=\min \left\{r s, \varepsilon^{2}, \bar{\rho} \bar{\rho}^{\prime}\right\}, \quad d:=\left\{r s, \varepsilon^{2}, \rho \rho^{\prime}\right\},
\end{aligned}
$$

we rewrite the assumptions in (D.142) as

$$
\frac{2^{7} \bar{c} G K s}{\mathfrak{a} \bar{d}}<1, \quad \frac{2^{7} c E K s}{\mathfrak{a} d}<1 .
$$

The inequality on the right clearly implies (D.144). So, we apply Lemma D.2 to the Hamiltonian (D.140), taking $r_{1}=\cdots=r_{\nu}=r, s_{1}=\cdots=s_{\nu}=s$, $\varepsilon_{1}=\cdots=\varepsilon_{\ell}=\varepsilon, \rho_{k}, \rho_{k}^{\prime}$ as in (D.145) and

$$
\begin{aligned}
& \hat{v}=\hat{v}_{0}:=v / 6, \quad \hat{s}=\hat{s}_{0}:=s / 6, \quad \hat{\varepsilon}=\hat{\varepsilon}_{0}:=\varepsilon / 6 \\
& \hat{\bar{v}}=\hat{\bar{v}}_{0}:=\bar{v} / 6, \quad \hat{\bar{s}}:=\hat{\bar{s}}_{0}:=\bar{s} / 6, \quad \hat{\bar{\varepsilon}}:=\hat{\bar{\varepsilon}}_{0}:=\bar{\varepsilon} / 6 \\
& \delta:=\left\{\hat{r} \hat{s}, \quad \hat{\varepsilon}^{2}, \hat{\rho} \hat{\rho}\right\}=\frac{d}{36} .
\end{aligned}
$$

Letting

$$
v_{1}:=v-2 \hat{v}_{0}=3 / 4 v, \quad s_{1}:=s-2 \hat{s}=2 / 3 s, \quad \varepsilon_{1}:=\varepsilon-2 \hat{\varepsilon}=2 / 3 \varepsilon
$$

by Lemma D.2, we find a canonical transformation $\Phi_{0}=X_{\phi_{0}}$ which is real-analytic on $W_{v_{1}, s_{1}, \varepsilon_{1}}$ and conjugates $H$ to $H_{1}=h+g+g_{1}+f_{1}$, where $g_{1}=\Pi_{\mathfrak{L} \cap \mathfrak{z}} T_{K} f$ and

${ }^{2}$ The time-one flow generated by $\phi$ is defined as the differential operator

$$
X_{\phi}^{1}:=\sum_{k=0}^{\infty} \frac{\mathrm{L}_{\phi}^{k}}{k !}
$$

where $\mathrm{L}_{\phi}^{0} f:=f$ and $\mathrm{L}_{\phi}^{k} f:=\left\{\phi, \mathrm{L}_{\phi}^{k-1} f\right\}$, with $k=1,2, \cdots$. 


$$
\begin{aligned}
\left\|f_{1}\right\|_{v_{1}, s_{1}, \varepsilon_{1}} & \leq\left(1-\frac{36 c E}{\mathfrak{a} d}\right)^{-1}\left[\frac{36 c E}{\mathfrak{a} d}+e^{-K s / 6}+\left(\frac{5}{6}\right)^{K}\right] E \\
& +\left(1-\frac{36 c E}{\mathfrak{a} d}\right)^{-1} \frac{36 \bar{c} G}{\mathfrak{a} \bar{d}} E
\end{aligned}
$$

where

Here, we have used

$$
\bar{\delta}:=\min \left\{\hat{r} \hat{s}, \hat{\varepsilon}^{2}, \hat{\bar{\rho}} \hat{\bar{\rho}}^{\prime}\right\}=\frac{\bar{d}}{36}
$$

$$
\begin{aligned}
\left\|\{\phi, g\}_{I, \varphi, \eta, \xi}\right\|_{v-\hat{v} . s-\hat{s}, \varepsilon-\hat{\varepsilon}} & =\left\|\{\phi, g\}_{I, \varphi, \eta^{1}, \xi^{1}}\right\|_{\bar{v}-\hat{v}, s-\hat{s}, \varepsilon-\hat{\varepsilon}} \\
& \leq \frac{\bar{c} G}{\mathfrak{a} \bar{\delta}}=36 \frac{\bar{c} G}{\mathfrak{a} \bar{d}}
\end{aligned}
$$

since $g$ depends on $\eta$, $\xi$ only via $\eta^{1}=\left(\eta_{1}, \cdots, \eta_{m_{1}}\right), \xi^{1}=\left(\xi_{1}, \cdots, \xi_{m_{1}}\right)$. It is sufficient to consider the case

$$
e^{-K s / 6}+\left(\frac{5}{6}\right)^{K} \leq \frac{18 c E}{\mathfrak{a} d}
$$

since otherwise the Lemma is proved. In such case, using (D.146) we can write

$$
\begin{aligned}
E_{1}=\left\|f_{1}\right\|_{v_{1}, s_{1}, \varepsilon_{1}} & \leq \frac{32}{23}\left(\frac{9}{32} \frac{2^{7} c E K s}{\mathfrak{a} d}+\frac{9}{64} \frac{2^{7} c E K s}{\mathfrak{a} d}+\frac{9}{32} \frac{2^{7} \bar{c} G K s}{\mathfrak{a} \bar{d}}\right) \frac{E}{K s} \\
& <\frac{E}{K s} \max \left\{\frac{2^{7} c E K s}{\mathfrak{a} d}, \frac{2^{7} \bar{c} G K s}{\mathfrak{a} \bar{d}}\right\}<\frac{E}{4}
\end{aligned}
$$

Let

$$
L:=\left[\frac{K s}{12 \log 2}\right]
$$

Note that

$$
L \geq 1, \quad K s>8 L,
$$

since we have assumed $K s \geq 12$. We want to prove that Lemma D.2 can be applied $L$ times with parameters

$$
\hat{v}_{i}=\frac{v}{4 L}, \quad \hat{\varepsilon}_{i}=\frac{\varepsilon}{4 L}, \quad \hat{s}_{i}=\frac{s}{4 L}, \quad \delta_{i}=\frac{d}{16 L^{2}}, \quad i=1, \cdots, L .
$$

For $L=1$, this follows from (D.148):

$$
E_{1}:=\left\|f_{1}\right\|_{v_{1}, s_{1}, \varepsilon_{1}} \leq \frac{E}{K s} \leq 2^{-7} \frac{\mathfrak{a} d}{c(K s)^{2}}<2^{-13} \frac{\mathfrak{a} \delta_{1}}{c}
$$

which is implied by the inequality in (D.148) and assumption (D.142). We then assume $L \geq 2$. Suppose, by induction, that, for a certain $1 \leq i \leq L-1$, and any $1 \leq j \leq i$, we have conjugated $H$ to

$$
H_{j}=\mathrm{h}+g+\bar{g}_{j}+f_{j}
$$

where $\bar{g}_{j}=\sum_{k=0}^{j-1} \Pi_{\mathfrak{L} \cap \mathfrak{Z}} T_{K} f_{k}$

$$
E_{j}:=\left\|f_{j}\right\|_{v_{j}, s_{j}, \varepsilon_{j}} \leq \min \left\{\frac{E}{4^{j}}, 2^{-6} \frac{\mathfrak{a} \delta_{j}}{c}\right\}
$$


where $\hat{v}_{0}, \hat{s}_{0}, \hat{\varepsilon}_{0}$ are as above, $v_{0}:=v, s_{0}:=s, \varepsilon_{0}:=\varepsilon$ and $v_{j}=v_{j-1}-2 \hat{v}_{j-1}$. Then by Lemma D.2, on the domain $W_{v_{j+1}, s_{j+1}, \varepsilon_{j+1}}$, we fined a real-analytic transformation $\Phi_{i}=X_{\phi_{i}}$, which conjugates $H_{i}$ to

$$
H_{i+1}=\mathrm{h}+g+\bar{g}_{i+1}+f_{i+1}
$$

where $\bar{g}_{i+1}=\bar{g}_{i}+\prod_{\mathfrak{L} \cap \mathfrak{K}} f_{i}=\sum_{k=0}^{i} \Pi_{\mathfrak{L} \cap \mathfrak{Z}} T_{K} f_{k}$. We prove that (D.151) is satisfied for $j=i+1$. Using ${ }^{3}$ the assumption on the right in (D.146) , (D.148), the inequality for $K s$ in (D.149) and the definition of $\delta_{i}$ in (D.150), we have

$$
\left\|\left\{\bar{g}_{i}, \phi_{i}\right\}\right\|_{v_{i}-\hat{v}_{i}, s_{i}-\hat{s}_{i}, \varepsilon_{i}-\hat{\varepsilon}_{i}} \leq\left[\frac{c}{\mathfrak{a} \delta_{i}}\left(E_{1}+\frac{E}{L}\right)\right] E_{i} \leq\left[\frac{c}{\mathfrak{a} \delta_{i}} \frac{E}{K s}+\frac{c}{\mathfrak{a} \delta_{i}} \frac{E}{L}\right] E_{i}<\frac{E_{i}}{32} .
$$

Moreover, by a similar argument as in (D.147) and since $g$ is actually real-analytic in the larger domain

$$
W_{\bar{v}, s, \varepsilon} \supset W_{\bar{v}_{i}-\hat{v}_{i}+\bar{v}, s_{i}-\hat{s}_{i}+\hat{s}, \varepsilon_{i}-\hat{\varepsilon}_{i}+\hat{\varepsilon}}
$$

we have

$$
\left\|\left\{g, \phi_{i}\right\}\right\|_{v_{i}-\hat{v}_{i}, s_{i}-\hat{s}_{i}, \varepsilon_{i}-\hat{\varepsilon}_{i}}=\left\|\left\{g, \phi_{i}\right\}\right\|_{\bar{v}_{i}-\hat{v}_{i}, s_{i}-\hat{s}_{i}, \varepsilon_{i}-\hat{\varepsilon}_{i}} \leq \frac{\bar{c} E_{i}}{\mathfrak{a} \bar{\delta}_{i}} \frac{G}{L}<\frac{E_{i}}{64},
$$

where

$$
\bar{\delta}_{i}:=\min \left\{\hat{r}_{i} \hat{s}_{i}, \overline{\hat{\rho}}_{i} \hat{\bar{\rho}}_{i}^{\prime}\right\}=\frac{\bar{d}}{16 L^{2}}, \quad i=1, \cdots, L
$$

Then we find

$$
\begin{aligned}
E_{i+1}=\left\|f_{i+1}\right\|_{v_{i+1}, s_{i+1}, \varepsilon_{i+1}} \leq & \left(1-\frac{c E_{i}}{\mathfrak{a} \delta_{1}}\right)^{-1}\left[\frac{c E_{i}}{\mathfrak{a} \delta_{1}}+e^{-K \hat{s}_{i}}+\left(\frac{\varepsilon_{i}-\hat{\varepsilon}_{i}}{\varepsilon_{i}}\right)^{K}\right] E_{i} \\
& +\left(1-\frac{c E_{i}}{\mathfrak{a} \delta_{1}}\right)^{-1}\left\|\left\{\bar{g}_{i}, \phi_{i}\right\}\right\|_{v_{i}-\hat{v}_{i}, s_{i}-\hat{s}_{i}, \varepsilon_{i}-\hat{\varepsilon}_{i}} \\
& +\left(1-\frac{c E_{i}}{\mathfrak{a} \delta_{1}}\right)^{-1}\left\|\left\{g, \phi_{i}\right\}\right\|_{\bar{v}_{i}-\overline{\hat{v}}_{i}, s_{i}-\hat{s}_{i}, \varepsilon_{i}-\hat{\varepsilon}_{i}} \\
& \leq \frac{64}{63}\left[\frac{1}{64}+\frac{1}{8}+\left(\frac{4}{7}\right)^{16}+\frac{1}{32}+\frac{1}{64}\right] E_{i} \\
& <\frac{E_{i}}{4}<E_{1}<2^{-6} \frac{\mathfrak{a} \delta_{1}}{c} .
\end{aligned}
$$

since $i \geq 1$. Then we let $\Phi:=\Phi_{0} \circ \cdots \circ \Phi_{L}, H_{*}:=H \circ \Phi=h+g+\bar{g}_{L+1}+f_{L+1}$, $g_{*}:=g_{L+1}, f_{*}:=f_{L+1}$ and we have, by telescopic inequalities and (D.148),

$$
\begin{aligned}
\left\|g_{*}-\Pi_{\mathfrak{L} \cap \mathfrak{K}} T_{K} f\right\|_{v / 6, s / 6, \varepsilon / 6} & =\sum_{i=1}^{L}\left\|\Pi_{\mathfrak{L} \cap \mathfrak{K}} T_{K} f_{i}\right\| \leq \sum_{i=1}^{L} E_{i} \leq E_{1} \sum_{i=1}^{L} \frac{1}{4^{i-1}} \\
& =\frac{4}{3} E_{1} \leq\left(\frac{2^{7} c E}{\mathfrak{a} d}+\frac{2^{7} \bar{c} G}{\mathfrak{a} \bar{d}}\right) E
\end{aligned}
$$

${ }^{3}$ For the proof of inequality $\left\|\left\{g_{i}, \phi_{i}\right\}\right\|_{v_{i}-\hat{v}_{i}, s_{i}-\hat{s}_{i}, \varepsilon_{i}-\hat{\varepsilon}_{i}} \leq \frac{c E_{i}}{\mathfrak{a} \delta_{1}}\left(E_{1}+\frac{E}{L}\right)$, compare 31 Proof of the Normal Form Lemma].

${ }^{4}$ Since $K>8 L$ and $L \geq 2$, one has $\left(1-\frac{3}{2 L}\right)^{K} \leq \frac{1}{\left(1+\frac{3}{2 L}\right)^{8 L}}$ with the r.h.s bounded above by $(4 / 7)^{16}$ (it decreases to $e^{-12}$ as $L \rightarrow+\infty$ ). 
Now we prove (D.143). Let $F \in W_{\bar{v}, s, \varepsilon}, F_{-1}:=F, F_{i}:=F \circ \Phi_{0} \circ \cdots \circ \Phi_{i}, i=0$, $\cdots, L$. Then

$$
\begin{aligned}
\|F \circ \Phi-F\|_{\bar{v} / 6, s / 6, \varepsilon / 6} & =\left\|F_{L}-F\right\|_{\bar{v}_{L+1}, s_{L+1}, \varepsilon_{L+1}} \\
& \leq \sum_{i=0}^{L}\left\|F_{i-1} \circ \Phi_{i}-F_{i-1}\right\|_{\bar{v}_{i+1}, s_{i+1}, \varepsilon_{i+1}} \\
& \leq \sum_{i=0}^{L} \frac{\frac{\bar{c} E_{i}}{\mathfrak{a} \bar{\delta}_{i}}}{\left(1-\frac{\bar{c} E_{i}}{\mathfrak{a} \delta_{i}}\right)}\|F\|_{\bar{v}_{i}, s_{i}, \varepsilon_{i}} \leq \frac{\sum_{i=0}^{L} \frac{\bar{c} E_{i}}{\mathfrak{a} \bar{\delta}_{i}}}{\prod_{i=0}^{L}\left(1-\frac{\bar{c} E_{i}}{\mathfrak{a} \bar{\delta}_{i}}\right)}\|F\|_{\bar{v}, s, \varepsilon} \\
& \leq \sum_{i=0}^{L} \frac{\bar{c} E_{i}}{\mathfrak{a} \bar{\delta}_{i}} e^{\frac{5}{4} \sum_{i=0}^{L} \frac{\bar{c} E_{i}}{\mathfrak{a} \delta_{i}}}\|F\|_{\bar{v}, s, \varepsilon} \leq \frac{2^{5} \bar{c} E_{0} K s}{\mathfrak{a} d}\|F\|_{\bar{v}, s, \varepsilon}
\end{aligned}
$$

where we have used $\frac{\bar{c} E_{i}}{\mathfrak{a} \bar{d}_{i}}<1 / 24$ that, for $0 \leq x \leq 1 / 24, \log (1-x)^{-1}<\frac{5}{4} x$ and

$$
\begin{aligned}
\sum_{i=0}^{L} \frac{\bar{c} E_{i}}{\mathfrak{a} \bar{\delta}_{i}} & =\frac{\bar{c} E_{0}}{\mathfrak{a} \bar{\delta}_{0}}+\sum_{i=1}^{L} \frac{\bar{c} E_{i}}{\mathfrak{a} \bar{\delta}_{i}} \leq \frac{2^{6} \bar{c} E_{0}}{\mathfrak{a} d}+\frac{\bar{c} E_{1}}{\mathfrak{a} \bar{\delta}_{1}} \sum_{i=1}^{L} \frac{1}{4^{i-1}} \\
& \leq \frac{2^{6} \bar{c} E_{0}}{\mathfrak{a} d}+\frac{4}{3} \frac{\bar{c} E_{1}}{\mathfrak{a} \bar{\delta}_{1}}<\frac{2^{4} \bar{c} E_{0} K s}{\mathfrak{a} d}
\end{aligned}
$$

The proof for $F \in W_{v, s, \varepsilon}$ is similar.

Proof of Proposition D.1. For simplicity of notations, we prove Proposition D.1 in the case $\nu=\ell=1$; the generalization to any $\nu, \ell$ being straightforward. Consider the Hamiltonian

$$
H_{0}\left(u_{1}, \varphi, p, q\right):=\mathrm{h}(I, p, q)+f_{1}\left(u_{1}, \varphi, p, q\right), \quad\left(u_{1}, \varphi, p, q\right) \in W_{v_{1}, s, \varepsilon}^{(1)} .
$$

To this Hamiltonian let us apply Lemma D.1, with $g \equiv 0$, so as to conjugate it to

$$
H_{1}:=H_{0} \circ \Phi_{1}=h+g_{1}+f_{* 1}^{(1)}, \quad\left(u_{1}, \varphi, p, q\right) \in W_{v_{1} / 6, s / 6, \varepsilon / 6}^{(1)}
$$

where $g_{1}, f_{* 1}^{(1)}$ correspond to $g_{*}, f_{*}$, hence satisfy

$$
\begin{aligned}
\left\|f_{* 1}^{(1)}\right\|_{v_{1} / 6, s / 6, \varepsilon / 6} & \leq e^{-K s / 6}\left\|f_{1}^{(i)}\right\|_{v_{1}, s, \varepsilon} \\
\left\|g_{1}\right\|_{v_{1} / 6, s / 6, \varepsilon / 6} & \leq \frac{7}{6}\left\|f_{1}\right\|_{v_{1}, s, \varepsilon} \\
\left\|g_{1}-\Pi_{\mathfrak{L} \cap \mathfrak{Z}} T_{K} f_{1}\right\|_{v_{1} / 6, s / 6, \varepsilon / 6} & \leq \frac{2^{7} c_{1}\left\|f_{1}\right\|_{v_{1}, s, \varepsilon}^{2}}{\mathfrak{a} d_{1}}
\end{aligned}
$$

Then we have

$$
H^{(1)}(u, \varphi, p, q):=H \circ \Phi_{1}=H_{0} \circ \Phi_{1}+\sum_{j=2}^{N} f_{j} \circ \Phi_{1}=\mathrm{h}+g_{1}+f_{1 *}^{(1)}+\sum_{j=2}^{N} f_{j}^{(1)}
$$

where $f_{j}^{(1)}:=f_{j} \circ \Phi_{1}$. Assume, inductively, that, for some $1 \leq i \leq N-1$ and any $1 \leq j \leq i$ we have conjugated $H$ to

$$
H^{(j)}(u, \varphi, p, q)=H \circ \Phi_{1} \circ \cdots \circ \Phi_{j}=\mathrm{h}+\sum_{k=1}^{j} g_{k}+\sum_{k=1}^{j} f_{k *}^{(j)}+\sum_{k=j+1}^{N} f_{k}^{(j)}
$$

where

$$
\Phi_{j}: W_{v / 6^{j}, s / 6^{j}, \varepsilon / 6^{j}}^{(j)} \rightarrow W_{v / 6^{j-1}, s / 6^{j-1}, \varepsilon / 6^{j-1}}^{(j-1)}
$$


transforms

$$
H_{j-1}:=\mathrm{h}+\sum_{k=1}^{j-1} g_{k}+f_{j}^{(j-1)}
$$

into

$$
H_{j-1} \circ \Phi_{j}=\mathrm{h}+\sum_{k=1}^{j} g_{k}+f_{* j}^{(j)}
$$

The Hamiltonian

$$
H_{i}\left(u_{i+1}, \varphi, p, q\right):=\mathrm{h}+\sum_{k=1}^{i} g_{k}\left(u_{k}, \varphi, p, q\right)+f_{i+1}^{(i)}\left(u_{i+1}, \varphi, p, q\right)
$$

is real-analytic for $\left(u_{i+1}, \varphi, p, q\right) \in W_{v_{i+1} / 6^{i}, s / 6^{i}, \varepsilon / 6^{i}}^{(i+1)}$ and satisfies the assumptions of Lemma D.1, with $\bar{N}=i$. Then one can find $\Phi_{i+1}: W_{v_{i+1} / 6^{i+1}, s / 6^{i+1}, \varepsilon / 6^{i+1}}^{(i+1)} \rightarrow$ $W_{v_{i+1} / 6^{i}, s / 6^{i}, \varepsilon / 6^{i}}^{(i+1)}$ such that $H_{i} \circ \Phi_{i+1}=\mathrm{h}+\sum_{k=1}^{i+1} g_{k}+f_{* i+1}^{(i+1)}$, where

$$
\begin{aligned}
& \left\|f_{* i+1}^{(i+1)}\right\|_{v_{i+1} / 6^{i+1}, s / 6^{i+1}, \varepsilon / 6^{i+1}} \\
& \leq e^{-K s / 6^{i+1}}\left\|f_{i+1}^{(i)}\right\|_{v_{i+1} / 6^{i}, s / 6^{i}, \varepsilon / 6^{i}} \\
& \leq\left(\frac{9}{8}\right)^{i} e^{-K s / 6^{i+1}}\left\|f_{i+1}\right\|_{v_{i+1}, s, \varepsilon} \\
& \left\|g_{i+1}\right\|_{v_{i+1} / 6^{i+1}, s / 6^{i+1}, \varepsilon / 6^{i+1}} \\
& \leq \frac{7}{6}\left\|f_{i+1}^{(i)}\right\|_{v_{i+1} / 6^{i}, s / 6^{i}, \varepsilon / 6^{i}} \leq \frac{7}{6}\left(\frac{9}{8}\right)^{i}\left\|f_{i+1}\right\|_{v_{i+1}, s, \varepsilon} \\
& \left\|g_{i+1}-\Pi_{\mathfrak{L} \cap \mathfrak{Z}} T_{K} f_{i+1}\right\|_{v_{i+1} / 6^{i+1}, s / 6^{i+1}, \varepsilon / 6^{i+1}} \\
& \leq\left\|g_{i+1}-\Pi_{\mathfrak{L} \cap \mathfrak{Z}} T_{K} f_{i+1}^{(i)}\right\|_{v_{i+1} / 6^{i+1}, s / 6^{i+1}, \varepsilon / 6^{i+1}} \\
& +\left\|\Pi_{\mathfrak{L} \cap \mathfrak{Z}} T_{K} f_{i+1}^{(i)}-\Pi_{\mathfrak{L} \cap \mathfrak{Z}} T_{K} f_{i+1}\right\|_{v_{i+1} / 6^{i+1}, s / 6^{i+1}, \varepsilon / 6^{i+1}} \\
& \leq\left\|g_{i+1}-\Pi_{\mathfrak{L} \cap \mathfrak{Z}} T_{K} f_{i+1}^{(i)}\right\|_{v_{i+1} / 6^{i+1}, s / 6^{i+1}, \varepsilon / 6^{i+1}} \\
& +\left\|f_{i+1}^{(i)}-f_{i+1}\right\|_{v_{i+1} / 6^{i+1}, s / 6^{i+1}, \varepsilon / 6^{i+1}} \\
& \leq\left(\frac{9}{8}\right)^{2 i} \frac{2^{7} c_{i+1}\left\|f_{i+1}\right\|_{v_{i+1}, s, \varepsilon}^{2}}{\mathfrak{a} d_{i+1}} \\
& +\frac{7}{6}\left(\frac{9}{8}\right)^{2 i} \sum_{j=1}^{i} \frac{2^{7} c_{j}\left\|f_{j}\right\|_{v_{j}, s, \varepsilon}}{\mathfrak{a} d_{j}}\left\|f_{i+1}\right\|_{v_{i+1}, s, \varepsilon} \\
& +\sum_{k=1}^{i}\left(\frac{9}{8}\right)^{i-k} \frac{2^{4} c_{k}\left\|f_{k}\right\|_{v_{k}, s, \varepsilon} K s}{\mathfrak{a} d_{k}}\left\|f_{i+1}\right\|_{v_{i+1}, s, \varepsilon}
\end{aligned}
$$


with $f_{k *}^{(i+1)}:=f_{k *}^{(i)} \circ \Phi_{i+1}$ for $1 \leq k \leq i+1$ and $f_{k}^{(i+1)}:=f_{k}^{(i)} \circ \Phi_{i+1}$ for $i+2 \leq k \leq N$. Then we find

$$
\begin{aligned}
H^{(i+1)} & :=H^{(i)} \circ \Phi_{i+1}=\left(\mathrm{h}+\sum_{k=1}^{i} g_{k}+\sum_{k=1}^{i} f_{k *}^{(i)}+\sum_{k=i+1}^{N} f_{k}^{(i)}\right) \circ \Phi_{i+1} \\
& =H_{i} \circ \Phi_{i+1}+\left(\sum_{k=1}^{i} f_{k *}^{(i)}+\sum_{k=i+2}^{N} f_{k}^{(i)}\right) \circ \Phi_{i+1} \\
& =\mathrm{h}+\sum_{k=1}^{i+1} g_{k}+\sum_{k=1}^{i+1} f_{k *}^{(i+1)}+\sum_{k=i+2}^{N} f_{k}^{(i+1)}
\end{aligned}
$$

and hence, after $N$ steps,

$$
H^{(N)}:=H \circ \Phi_{1} \cdots \circ \Phi_{N}=\mathrm{h}+\sum_{k=1}^{N} g_{k}+\sum_{k=1}^{i+1} f_{k *}^{(N)}
$$

satisfies the thesis of Proposition D.1

\section{D.2. A slightly-perturbed integrable system}

The following result is well known in the literature of close-to be integrable systems, hence its proof is omitted. Note that it deals with an integrable system, close to another integrable one.

THEOREM D.1. One can find a number $\mathfrak{c}_{0}$ such that, for any real-analytic, one-dimensional, system

$$
\mathrm{H}(P, Q)=\mathrm{h}\left(\frac{P^{2}+Q^{2}}{2}\right)+f(P, Q) \quad(P, Q) \in \mathfrak{B}=B_{\varepsilon}^{2}(0) \subset \mathbb{C}^{2}
$$

and any $0<\bar{\varepsilon}<\varepsilon$, such that

$$
\inf _{B_{\varepsilon}^{2}}|\partial \mathrm{h}| \geq \mathfrak{a}, \quad \sup _{B_{\varepsilon}^{2}}|f| \leq \mathfrak{e}, \quad \frac{1}{\mathfrak{c}_{0}} \frac{\mathfrak{e}}{\mathfrak{a} \bar{\varepsilon}^{2}}<1,
$$

one can find a real-analytic transformation

$$
\phi_{*}: \quad\left(P_{*}, Q_{*}\right) \in B_{\varepsilon-\bar{\varepsilon}}^{2} \rightarrow(P, Q) \in B_{\varepsilon}^{2}
$$

which conjugates $\mathrm{H}$ to a function $\mathrm{H}_{*}=\mathrm{H} \circ \phi_{*}$ depending only on $\frac{P_{*}^{2}+Q_{*}^{2}}{2}$. The assertion can be extended to the case that $\mathrm{h}, f$ are functions of other canonical coordinates $\left(P^{\prime}, Q^{\prime}, \mathrm{y}, \mathrm{x}\right)$, depending on them only via $\mathrm{Y}=\left(\mathrm{y}, \frac{P_{1}^{\prime 2}+{Q_{1}^{\prime}}^{2}}{2}, \cdots, \frac{P_{m}^{\prime 2}+Q_{m}^{\prime}{ }^{2}}{2}\right)$, with $\mathrm{y} \in \mathrm{Y}_{\rho},\left(P_{j}^{\prime}, Q_{j}^{\prime}\right) \in B_{\varepsilon_{j}^{\prime}}^{2}$. In this case, letting $\left(P_{*}, Q_{*}\right) \rightarrow \phi_{*}\left(P_{*}, Q_{*} ; \mathrm{Y}\right)$ the transformation obtained for any fixed value of $\mathrm{Y}$, there exists a canonical, realanalytic, transformation $\Phi_{*}$ of the form

$\Phi_{*}:(P, Q)=\phi_{*}\left(P_{*}, Q_{*} ; \mathrm{Y}_{*}\right) \mathrm{y}=\mathrm{y}_{*}, \mathrm{x}=\mathrm{x}_{*}+\varphi\left(\mathrm{Y}_{*}\right), P_{j}^{\prime}+\mathrm{i} Q_{j}^{\prime}=e^{\mathrm{i} \psi_{j}\left(\mathrm{Y}_{*}\right)}\left(P_{* j}^{\prime}+\mathrm{i} Q_{* j}^{\prime}\right)$

which conjugates $\mathrm{H}$ to a function $\mathrm{H}_{*}=\mathrm{H} \circ \Phi_{*}$ depending only on $\frac{P_{*}^{2}+Q_{*}^{2}}{2}$ and $\mathrm{Y}_{*}$. In this case, the functions $\varphi_{j}, \psi_{j}$ verify

$$
\left|\varphi_{j}\right| \leq \frac{1}{\mathfrak{c}_{0}} \frac{\mathfrak{e}}{\mathfrak{a} \rho_{j}}, \quad\left|\psi_{j}\right| \leq \frac{1}{\mathfrak{c}_{0}} \frac{\mathfrak{e}}{\mathfrak{a} \varepsilon_{j}^{\prime 2}}
$$




\section{APPENDIX E}

\section{More on the geometrical structure of the $\mathcal{P}$-coordinates, compared to Deprit's coordinates}

In this chapter we aim to point out differences and similarities between the $\mathcal{P}$-coordinates and the coordinates denoted as $(\Psi, \Gamma, \Lambda, \psi, \gamma, \ell)$ in $\mathbf{7}, \mathbf{9}, \mathbf{2 7}$.

We recall that the "planetary" coordinates $(\Psi, \Gamma, \Lambda, \psi, \gamma, \ell)$ may be derived (after a canonical transformation) from a more general set of canonical coordinates studied by A. Deprit. In their planetary form, the coordinates $(\Psi, \Gamma, \Lambda, \psi, \gamma, \ell)$ have been rediscovered 1 by the author during her $\mathrm{PhD}$, under the strong motivation of their application to the planetary problem $[\mathbf{9}, \mathbf{2 7}]$.

Let us recall their definition2, in the spirit of Kepler maps (Definition 2.2).

Let $\mathrm{C}_{\mathcal{E}}^{(i)}, \mathrm{S}_{\mathcal{E}}^{(i)}$ be as in (2.8) of Chapter 2 and define the $\mathcal{D} e p$-nodes

$$
n_{i}:= \begin{cases}k^{(3)} \times \mathrm{S}_{\mathcal{E}}^{(1)} & i=0 \\ \mathrm{~S}_{\mathcal{E}}^{(i)} \times \mathrm{S}_{\mathcal{E}}^{(i+1)}=-\mathrm{S}_{\mathcal{E}}^{(i)} \times \mathrm{C}_{\mathcal{E}}^{(i)} & i=1, \cdots, n-1 . \\ -n_{n-1} & i=n\end{cases}
$$

Then let

$\mathcal{E}_{\mathcal{D} e p}:=\left\{\left(\left(\mathfrak{E}_{1}, \cdots, \mathfrak{E}_{n}\right) \subset E^{3} \times \cdots \times E^{3}\right): \quad 0<e_{i}<1, \quad n_{i-1} \neq 0 \quad \forall i=1, \cdots, n\right\}$.

On $\mathcal{E}_{\mathcal{D} e p}$, define the map

$$
\tau_{\mathcal{D} e p}^{-1}: \quad\left(\mathfrak{E}_{1}, \cdots, \mathfrak{E}_{n}\right) \in \mathcal{E}_{\mathcal{D} e p} \rightarrow \mathrm{X}_{\mathcal{D} e p} \in \mathfrak{X}_{\mathcal{D} e p}=\tau_{\mathcal{D} e p}^{-1}\left(\mathcal{E}_{\mathcal{D} e p}\right)
$$

where

$$
\mathrm{X}_{\mathcal{D} e p}=(\Psi, \Gamma, \Lambda, \psi, \gamma) \in \mathbb{R}^{n} \times \mathbb{R}_{+}^{n} \times \mathbb{R}_{+}^{n} \times \mathbb{T}^{n} \times \mathbb{T}^{n}
$$

where

$$
\begin{array}{ll}
\Psi=\left(\Psi_{-1}, \Psi_{0}, \bar{\Psi}\right) \in \mathbb{R}_{+} \times \mathbb{R}_{+} \times \mathbb{R}_{+}^{n-2} & \psi=\left(\psi_{-1}, \psi_{0}, \bar{\Psi}\right) \in \mathbb{T} \times \mathbb{T} \times \mathbb{T}^{n-2} \\
\Gamma=\left(\Gamma_{1}, \cdots, \Gamma_{n}\right) \in \mathbb{R}_{+}^{n} & \gamma=\left(\gamma_{1}, \cdots, \gamma_{n}\right) \in \mathbb{T}^{n} \\
\Lambda=\left(\Lambda_{1}, \cdots, \Lambda_{n}\right) \in \mathbb{R}_{+}^{n} &
\end{array}
$$

with

$$
\bar{\Psi}=\left(\Psi_{1}, \cdots, \Psi_{n-2}\right) \quad \bar{\psi}=\left(\psi_{1}, \cdots, \psi_{n-2}\right)
$$

${ }^{1}$ The proof of their symplectic character found in [27 has been published in $\mathbf{7}$. Another proof has been given in $\mathbf{3 6}$.

${ }^{2}$ For sake of uniformity, we use slightly different notations with respect to the ones in $\mathbf{7}$, actually closer to the ones of the paper [12]). 
are defined as follows. The coordinates $\Lambda_{j}$ are as in (2.11), while $(\Psi, \Gamma, \psi, \gamma)$ are defined as

$$
\begin{aligned}
& \Psi_{i-2}=\left\{\begin{array}{ll}
Z:=\mathrm{S}_{\mathcal{E}}^{(1)} \cdot k^{(3)} \\
\left|\mathrm{S}_{\mathcal{E}}^{(i)}\right|
\end{array} \quad \psi_{i-2}= \begin{cases}\zeta:=\alpha_{k^{(3)}}\left(k^{(1)}, n_{0}\right) & i=1 \\
\alpha_{\mathrm{S}_{\mathcal{E}}^{(i-1)}}\left(n_{i-2}, n_{i-1}\right) & 2 \leq i \leq n\end{cases} \right. \\
& \Gamma_{i}:=\left|\mathrm{C}_{\mathcal{E}}^{(i)}\right| \quad \gamma_{i}:=\alpha_{\mathrm{C}_{\mathcal{E}}^{(i)}}\left(n_{i}, P^{(i)}\right) \quad 1 \leq i \leq n
\end{aligned}
$$

Then $\tau_{\mathcal{D} e p}^{-1}$ is a bijection $[\mathbf{7}, \mathbf{1 2}, \mathbf{2 7}, \mathbf{3 6}$.

Definition E.1. We call Deprit's map, or Dep map, the Kepler map

$$
\text { Dep }: \quad \text { Dep }=\left(\mathrm{X}_{\mathcal{D} e p}, \ell\right) \in \mathcal{D}_{\mathcal{D} e p}=\mathfrak{X}_{\mathcal{D} e p} \times \mathbb{T}^{n} \rightarrow(y, x) \in \mathbb{R}^{3 n} \times \mathbb{R}^{3 n}
$$

associated to $\tau_{\mathcal{D} e p}$

\section{Comparing $\mathcal{P}$ and $\mathcal{D} e p$}

a) Both the $\mathcal{P}$ and $\mathcal{D} e p$-coordinates reduce the system to $(3 n-2)$ degrees of freedom. They share the following three coordinates (two actions and an angle)

$$
\Psi_{-1}=Z=\Theta_{0}, \quad \psi_{-1}=\zeta=\vartheta_{0}, \quad \Psi_{0}=\mathrm{G}=\chi_{0}
$$

which are integrals of the system. As a consequence, the coordinates $(Z, \zeta)$ and, respectively,

$$
\mathrm{g}:=\psi_{0}, \quad \mathfrak{g}:=\kappa_{0}
$$

do not appear into the Hamiltonian. Note that $\mathcal{D} e p$ and $\mathcal{P}$ share also the fixed node $n_{0}=\nu_{1}$.

b) The angle $g$ for the set $\mathcal{D} e p$ describes the motion of the node $n_{1}$ in (E.153) and, by the cyclic character of $g$, this motion is negligible. Its counterpart in the set $\mathcal{P}$ is the node $\mathrm{n}_{1}$ in (2.10), the negligible motion of which is governed by $\mathfrak{g}$.

c) Compare the diagrams in (2.20) and (2.21) with the two ones associated to the Dep-map, respectively: 


$$
\begin{aligned}
& n_{0} \quad n_{1} \quad \vdots \quad n_{n-2} \quad n_{n-1} \\
& \Uparrow \quad \Uparrow \quad \begin{array}{llll}
\Uparrow & \Uparrow
\end{array} \\
& k^{(3)} \rightarrow \mathrm{S}_{\mathcal{E}}^{(1)} \rightarrow \mathrm{S}_{\mathcal{E}}^{(2)} \rightarrow \cdots \rightarrow \mathrm{S}_{\mathcal{E}}^{(n-1)} \rightarrow \mathrm{S}_{\mathcal{E}}^{(n)}=\mathrm{C}_{\mathcal{E}}^{(n)} \\
& \downarrow \quad \downarrow \quad \vdots \quad \downarrow \\
& \mathrm{C}_{\mathcal{E}}^{(1)} \quad \mathrm{C}_{\mathcal{E}}^{(2)} \quad \vdots \quad \mathrm{C}_{\mathcal{E}}^{(n-1)} \\
& \Downarrow \quad \Downarrow \quad \vdots \quad \Downarrow \\
& \begin{array}{llll}
-n_{1} & -n_{2} & \vdots & -n_{n-1}
\end{array}
\end{aligned}
$$

and

$$
\begin{array}{cccccccc}
\mathrm{F}_{0} \rightarrow & \mathrm{F}_{1}^{*} \rightarrow & \cdots & \mathrm{F}_{i}^{*} & \rightarrow & \cdots & \rightarrow & \mathrm{F}_{n}^{*}=\mathrm{G}_{n}^{*} \\
\downarrow & \vdots & & \downarrow & \vdots & \downarrow \\
\mathrm{G}_{1}^{*} & & & \mathrm{G}_{i}^{*} & & & \mathrm{G}_{n}^{*}
\end{array}
$$

where

$$
\mathrm{F}_{i}^{*}=\left(n_{i-1}, \cdot, \mathrm{S}_{\mathcal{E}}^{(i)}\right) \quad \mathrm{G}_{i}^{*}=\left(-n_{i}, \cdot, \mathrm{C}_{\mathcal{E}}^{(i)}\right) \quad i=1, \cdots, n .
$$

Note that, analogously to (2.20),$n_{i}$ in (E.153) is the skew-product of its two previous vectors in the tree (2.20).

d) While $\mathcal{D} e p$ is not defined for the planar problem, $\mathcal{P}$ is, and, in that case, the coordinates $(\Theta, \chi, \vartheta, \kappa)$ in (2.11) reduce to 3

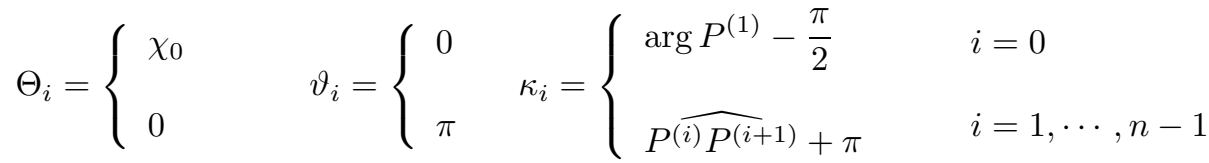

$$
\begin{aligned}
& \chi_{i}=\sum_{j=i+1}^{n}\left\|\mathrm{C}_{\mathcal{E}}^{(j)}\right\|
\end{aligned}
$$

while the $(\Lambda, \ell)$ remain unchanged.

e) The $\mathcal{P}$-map is singular when some eccentricity $e_{i}$ vanishes or some of the following relations hold

$$
\mathrm{S}_{\mathcal{E}}^{(1)}\left\|k^{(3)} \quad P^{(i)}\right\| \mathrm{S}_{\mathcal{E}}^{(i)} \quad \mathrm{S}_{\mathcal{E}}^{(i+1)} \| P^{(i)} .
$$

${ }^{3}$ Here by "planar case" we mean $\mathrm{C}_{\mathcal{E}}^{(1)}\|\cdots\| \mathrm{C}_{\mathcal{E}}^{(n)} \| k^{(3)}$. Note that, to be more precise, $\vartheta_{0}$ and $\kappa_{0}$ would not exist in that case (since $\nu_{1}=0$ ). However, since they are both cyclic angles, we can fix them to an arbitrary value. The choice above corresponds to replace $\nu_{1}$ with $k^{(1)}$. 
The former of such relations is negligible, while the other ones have no physical meaning. Therefore, the only physically relevant singularities of $\mathcal{P}$ are for zeroeccentric motions.

The $\mathcal{D} e p$-map is singular when some eccentricity $e_{i}$ vanishes or some of the following relations hold

$$
\mathrm{S}_{\mathcal{E}}^{(1)}\left\|k^{(3)} \quad \mathrm{S}_{\mathcal{E}}^{(i+1)}\right\| \mathrm{S}_{\mathcal{E}}^{(i)} \quad i=1, \cdots, n-1 .
$$

The configurations $\mathrm{S}_{\mathcal{E}}^{(i)} \| \mathrm{S}_{\mathcal{E}}^{(i+1)}$ have a relevant physical meaning, since the planar case corresponds to the intersection of all such configurations. A complete regularization of all the singularities of the Dep-map has been obtained in $\mathbf{9}, \mathbf{2 7}$, which allowed to overcome the problem of the rotational degeneracy (see $\mathbf{8}$ for information) of the planetary problem and to construct the Brkhoff normal form of it. It works at expenses of one extra-degree of freedom.

f) The Euclidean lengths $\left\|\mathrm{C}_{\mathcal{E}}^{(i)}\right\|$ of the planets' angular momenta are the actions $\Gamma_{i}$ among $\mathcal{D} e p$-coordinates: see (E.154). In terms of the $\mathcal{P}$-coordinates they have more involved expressions in (2.17). As mentioned in the previous item, this makes more difficult regularizing singular configurations with zero eccentricity. The formula simplifies in the planar case:

$$
\left\|\mathrm{C}_{\mathcal{E}}^{(i)}\right\|= \begin{cases}\left|\chi_{i-1}-\chi_{i}\right| & i=1, \cdots, n-1 \\ \chi_{n-1} & i=n\end{cases}
$$

where $|w|:=\sqrt{w^{2}}$, for a given $w \in \mathbb{C}$.

g) Reflections are not well described in the framework of the Dep-reduction: Compare, e.g., [29, Section 4.4]. Instead, in the framework of the $\mathcal{P}$-reduction, the transformation

$$
(\bar{\Theta}, \bar{\vartheta}) \rightarrow(-\bar{\Theta}, 2 k \pi-\bar{\vartheta}) \quad k \in \mathbb{Z}^{n-1}
$$

corresponds to changing the sign of the second component of any $y^{(i)}$ and any $x^{(i)}$. Therefore, any of the points

$$
(\bar{\Theta}, \bar{\vartheta})=(0, k \pi) \quad k \in \mathbb{Z}^{n-1}
$$

is an equilibrium point for the Hamiltonian, corresponding to a co-planar configuration. Compare Proposition 2.2. 


\section{Bibliography}

[1] V. I. Arnol'd, Proof of a theorem of A. N. Kolmogorov on the preservation of conditionally periodic motions under a small perturbation of the Hamiltonian (Russian), Uspehi Mat. Nauk 18 (1963), no. 5 (113), 13-40. MR0163025

[2] V. I. Arnol'd, Small denominators and problems of stability of motion in classical and celestial mechanics (Russian), Uspehi Mat. Nauk 18 (1963), no. 6 (114), 91-192. MR0170705

[3] F. Boigey, Élimination des nouds dans le problème newtonien des quatre corps (French, with English summary), Celestial Mech. 27 (1982), no. 4, 399-414, DOI 10.1007/BF01228562. MR670321

[4] A. Celletti and G. Pinzari, Four classical methods for determining planetary elliptic elements: a comparison, Celestial Mech. Dynam. Astronom. 93 (2005), no. 1-4, 1-52, DOI 10.1007/s10569-005-8663-8. MR2186799

[5] L. Chierchia. The Planetary N-Body Problem. UNESCO Encyclopedia of Life Support Systems, 6.119.55, 2012.

[6] L. Chierchia and G. Pinzari, Properly-degenerate KAM theory (following V. I. Arnold), Discrete Contin. Dyn. Syst. Ser. S 3 (2010), no. 4, 545-578, DOI 10.3934/dcdss.2010.3.545. MR2684064

[7] L. Chierchia and G. Pinzari, Deprit's reduction of the nodes revisited, Celestial Mech. Dynam. Astronom. 109 (2011), no. 3, 285-301, DOI 10.1007/s10569-010-9329-8. MR2777135

[8] L. Chierchia and G. Pinzari, Planetary Birkhoff normal forms, J. Mod. Dyn. 5 (2011), no. 4, 623-664. MR 2903753

[9] L. Chierchia and G. Pinzari, The planetary $N$-body problem: symplectic foliation, reductions and invariant tori, Invent. Math. 186 (2011), no. 1, 1-77, DOI 10.1007/s00222-011-0313-z. MR2836051

[10] L. Chierchia and G. Pinzari. Metric stability of the planetary n-body problem. Proceedings of the International Congress of Mathematicians, 2014.

[11] A. Delshams, V. Kaloshin, A. de la Rosa, and T. M. Seara. Global instability in the elliptic restricted three body problem. arXiv: 1501.01214, 2015.

[12] A. Deprit, Elimination of the nodes in problems of $n$ bodies, Celestial Mech. 30 (1983), no. 2, 181-195, DOI 10.1007/BF01234305. MR711657

[13] J. Féjoz. Work in progress.

[14] J. Féjoz, Démonstration du 'théorème d'Arnold' sur la stabilité du système planétaire (d'après Herman) (French, with English and French summaries), Ergodic Theory Dynam. Systems 24 (2004), no. 5, 1521-1582, DOI 10.1017/S0143385704000410. MR2104595

[15] J. Féjoz, On action-angle coordinates and the Poincaré coordinates, Regul. Chaotic Dyn. 18 (2013), no. 6, 703-718, DOI 10.1134/S1560354713060105. MR 3146588

[16] J. Féjoz. On "Arnold's theorem" in celestial mechanics -a summary with an appendix on the poincaré coordinates. Discrete and Continuous Dynamical Systems, 33:3555-3565, 2013.

[17] J. Fejoz, M. Guardia, V. Kaloshin, and P. Roldan. Kirkwood gaps and diffusion along mean motion resonances in the restricted planar three body problem. J. Eur. Math. Soc., 2014.

[18] S. Ferrer and C. Osácar, Harrington's Hamiltonian in the stellar problem of three bodies: reductions, relative equilibria and bifurcations, Celestial Mech. Dynam. Astronom. 58 (1994), no. 3, 245-275, DOI 10.1007/BF00691977. MR1271458

[19] R. S. Harrington. The stellar three-body problem. Celestial Mech. and Dyn. Astronomy, 1(2):200-209, 1969.

[20] M. R. Herman. Torsion du problème planétaire, edited by J. Féjoz in 2009. Available in the electronic 'Archives Michel Herman' at http://www.college-de-france.fr/default/ EN/all/equ_dif/archives_michel_herman.htm. 
[21] C. G. J. Jacobi. Sur l'élimination des noeuds dans le problème des trois corps. Astronomische Nachrichten, Bd XX:81-102, 1842.

[22] A. N. Kolmogorov, On conservation of conditionally periodic motions for a small change in Hamilton's function (Russian), Dokl. Akad. Nauk SSSR (N.S.) 98 (1954), 527-530. MR.0068687

[23] J. Laskar and P. Robutel, Stability of the planetary three-body problem. I. Expansion of the planetary Hamiltonian, Celestial Mech. Dynam. Astronom. 62 (1995), no. 3, 193-217, DOI 10.1007/BF00692088. MR 1364477

[24] T. Levi-Civita. Sopra la equazione di Kepler. Astronomische Nachrichten, 165(20):313-314, 1904.

[25] F. Malige, P. Robutel, and J. Laskar, Partial reduction in the $n$-body planetary problem using the angular momentum integral, Celestial Mech. Dynam. Astronom. 84 (2002), no. 3, 283-316, DOI 10.1023/A:1020392219443. MR:1935017

[26] J. Moser, On invariant curves of area-preserving mappings of an annulus, Nachr. Akad. Wiss. Göttingen Math.-Phys. Kl. II 1962 (1962), 1-20. MR0147741

[27] G. Pinzari. On the Kolmogorov set for many-body problems. PhD thesis, Università Roma Tre, April 2009.

[28] G. Pinzari, Aspects of the planetary Birkhoff normal form, Regul. Chaotic Dyn. 18 (2013), no. 6, 860-906, DOI 10.1134/S1560354713060178. MR3146595

[29] G. Pinzari, Canonical coordinates for the planetary problem, Acta Appl. Math. 137 (2015), 205-232, DOI 10.1007/s10440-014-9996-7. MR3343379

[30] H. Poincaré. Les méthodes nouvelles de la mécanique céleste. Gauthier-Villars, Paris, 1892.

[31] J. Pöschel, Nekhoroshev estimates for quasi-convex Hamiltonian systems, Math. Z. 213 (1993), no. 2, 187-216, DOI 10.1007/BF03025718. MR1221713

[32] R. Radau, Sur une transformation des équations différentielles de la dynamique (French), Ann. Sci. École Norm. Sup. 5 (1868), 311-375. MR1508550

[33] P. Robutel, Stability of the planetary three-body problem. II. KAM theory and existence of quasiperiodic motions, Celestial Mech. Dynam. Astronom. 62 (1995), no. 3, 219-261, DOI 10.1007/BF00692089. MR 1364478

[34] H. Rüssmann, Invariant tori in non-degenerate nearly integrable Hamiltonian systems, Regul. Chaotic Dyn. 6 (2001), no. 2, 119-204, DOI 10.1070/RD2001v006n02ABEH000169. MR 1843664

[35] F. Tisserand. Traité de mécanique céleste. Gauthier-Villars, I, 1889-1896

[36] L. Zhao, Partial reduction and Delaunay/Deprit variables, Celestial Mech. Dynam. Astronom. 120 (2014), no. 4, 423-432, DOI 10.1007/s10569-014-9584-1. MR.3277249 


\section{Editorial Information}

To be published in the Memoirs, a paper must be correct, new, nontrivial, and significant. Further, it must be well written and of interest to a substantial number of mathematicians. Piecemeal results, such as an inconclusive step toward an unproved major theorem or a minor variation on a known result, are in general not acceptable for publication.

Papers appearing in Memoirs are generally at least 80 and not more than 200 published pages in length. Papers less than 80 or more than 200 published pages require the approval of the Managing Editor of the Transactions/Memoirs Editorial Board. Published pages are the same size as those generated in the style files provided for $\mathcal{A} \mathcal{M} \mathcal{S}$ - $\mathrm{L}$ TEX $\mathrm{T}$ or $\mathcal{A} \mathcal{M} \mathcal{S}$ - $\mathrm{TE}_{\mathrm{E}} \mathrm{X}$.

Information on the backlog for this journal can be found on the AMS website starting from http://www. ams.org/memo.

A Consent to Publish is required before we can begin processing your paper. After a paper is accepted for publication, the Providence office will send a Consent to Publish and Copyright Agreement to all authors of the paper. By submitting a paper to the Memoirs, authors certify that the results have not been submitted to nor are they under consideration for publication by another journal, conference proceedings, or similar publication.

\section{Information for Authors}

Memoirs is an author-prepared publication. Once formatted for print and on-line publication, articles will be published as is with the addition of AMS-prepared frontmatter and backmatter. Articles are not copyedited; however, confirmation copy will be sent to the authors.

Initial submission. The AMS uses Centralized Manuscript Processing for initial submissions. Authors should submit a PDF file using the Initial Manuscript Submission form found at www . ams.org/submission/memo, or send one copy of the manuscript to the following address: Centralized Manuscript Processing, MEMOIRS OF THE AMS, 201 Charles Street, Providence, RI 02904-2294 USA. If a paper copy is being forwarded to the AMS, indicate that it is for Memoirs and include the name of the corresponding author, contact information such as email address or mailing address, and the name of an appropriate Editor to review the paper (see the list of Editors below).

The paper must contain a descriptive title and an abstract that summarizes the article in language suitable for workers in the general field (algebra, analysis, etc.). The descriptive title should be short, but informative; useless or vague phrases such as "some remarks about" or "concerning" should be avoided. The abstract should be at least one complete sentence, and at most 300 words. Included with the footnotes to the paper should be the 2010 Mathematics Subject Classification representing the primary and secondary subjects of the article. The classifications are accessible from ww. ams.org/msc/. The Mathematics Subject Classification footnote may be followed by a list of key words and phrases describing the subject matter of the article and taken from it. Journal abbreviations used in bibliographies are listed in the latest Mathematical Reviews annual index. The series abbreviations are also accessible from www. ams.org/msnhtml/serials.pdf. To help in preparing and verifying references, the AMS offers MR Lookup, a Reference Tool for Linking, at www.ams.org/mrlookup/.

Electronically prepared manuscripts. The AMS encourages electronically prepared manuscripts, with a strong preference for $\mathcal{A} \mathcal{M} \mathcal{S}-\mathrm{L}_{\mathrm{A}} \mathrm{T}_{\mathrm{E}} \mathrm{X}$. To this end, the Society has prepared $\mathcal{A} \mathcal{M S}$-LATEX author packages for each AMS publication. Author packages include instructions for preparing electronic manuscripts, samples, and a style file that generates the particular design specifications of that publication series. Though $\mathcal{A M S}_{\mathcal{M}}$ - $\mathrm{L}_{\mathrm{A}} \mathrm{T}_{\mathrm{E}} \mathrm{X}$ is the highly preferred format of $\mathrm{T}_{\mathrm{E} X}$, author packages are also available in $\mathcal{A M}_{\mathcal{M}}$ - $\mathrm{T}_{\mathrm{E}} \mathrm{X}$.

Authors may retrieve an author package for Memoirs of the AMS from www.ams.org/ journals/memo/memoauthorpac.html. The AMS Author Handbook is available in PDF format from the author package link. The author package can also be obtained free 
of charge by sending email to tech-support@ams.org or from the Publication Division, American Mathematical Society, 201 Charles St., Providence, RI 02904-2294, USA. When requesting an author package, please specify $\mathcal{A M}_{\mathcal{M}} \mathrm{S}_{\mathrm{A}} \mathrm{T}_{\mathrm{E}} \mathrm{X}$ or $\mathcal{A M}_{\mathcal{S}} \mathrm{S}_{\mathrm{E}} \mathrm{X}$ and the publication in which your paper will appear. Please be sure to include your complete mailing address.

After acceptance. The source files for the final version of the electronic manuscript should be sent to the Providence office immediately after the paper has been accepted for publication. The author should also submit a PDF of the final version of the paper to the editor, who will forward a copy to the Providence office.

Accepted electronically prepared files can be submitted via the web at www.ams.org/ submit-book-journal/, sent via FTP, or sent on CD to the Electronic Prepress Department, American Mathematical Society, 201 Charles Street, Providence, RI 02904-2294 USA. TEX source files and graphic files can be transferred over the Internet by FTP to the Internet node ftp.ams.org (130.44.1.100). When sending a manuscript electronically via $C D$, please be sure to include a message indicating that the paper is for the Memoirs.

Electronic graphics. Comprehensive instructions on preparing graphics are available at www.ams.org/authors/journals.html. A few of the major requirements are given here.

Submit files for graphics as EPS (Encapsulated PostScript) files. This includes graphics originated via a graphics application as well as scanned photographs or other computergenerated images. If this is not possible, TIFF files are acceptable as long as they can be opened in Adobe Photoshop or Illustrator.

Authors using graphics packages for the creation of electronic art should also avoid the use of any lines thinner than 0.5 points in width. Many graphics packages allow the user to specify a "hairline" for a very thin line. Hairlines often look acceptable when proofed on a typical laser printer. However, when produced on a high-resolution laser imagesetter, hairlines become nearly invisible and will be lost entirely in the final printing process.

Screens should be set to values between $15 \%$ and $85 \%$. Screens which fall outside of this range are too light or too dark to print correctly. Variations of screens within a graphic should be no less than $10 \%$.

Any graphics created in color will be rendered in grayscale for the printed version unless color printing is authorized by the Managing Editor and the Publisher. In general, color graphics will appear in color in the online version.

Inquiries. Any inquiries concerning a paper that has been accepted for publication should be sent to memo-query@ams . org or directly to the Electronic Prepress Department, American Mathematical Society, 201 Charles St., Providence, RI 02904-2294 USA. 


\section{Editors}

This journal is designed particularly for long research papers, normally at least 80 pages in length, and groups of cognate papers in pure and applied mathematics. Papers intended for publication in the Memoirs should be addressed to one of the following editors. The AMS uses Centralized Manuscript Processing for initial submissions to AMS journals. Authors should follow instructions listed on the Initial Submission page found at www.ams.org/memo/memosubmit.html.

1. GEOMETRY, TOPOLOGY \& LOGIC

Coordinating Editor: Richard Canary, Department of Mathematics, University of Michigan, Ann Arbor, MI 48109-1043 USA; e-mail: canary@umich.edu

Algebraic topology, Michael Hill, Department of Mathematics, University of California Los Angeles, Los Angeles, CA 90095 USA; e-mail: mikehill@math.ucla.edu

Differential geometry, Chiu-Chu Melissa Liu, Department of Mathematics, Columbia University, New York, NY 10027 USA; e-mail: ccliu@math.columbia.edu

Logic, Noam Greenberg, School of Mathematics and Statistics, Victoria University of Wellington, Wellington 6140, New Zealand; e-mail: greenberg@msor.vuw.ac.nz

Low-dimensional topology and geometric structures, Richard Canary

2. ALGEBRA AND NUMBER THEORY

Coordinating Editor: Henri Darmon, Department of Mathematics, McGill University, Montreal, Quebec H3A 0G4, Canada; e-mail: darmon@math.mcgill.ca

Algebra, Michael Larsen, Department of Mathematics, Rawles Hall, Indiana University, 831 E 3rd St., Bloomington, IN 47405 USA; e-mail: mjlarsen@indiana.edu

Algebraic geometry, Lucia Caporaso, Department of Mathematics and Physics, Roma Tre University, Largo San Leonardo Murialdo, I-00146 Rome, Italy; e-mail: LCedit@mat.uniroma3.it

Arithmetic geometry, Ted C. Chinburg, Department of Mathematics, University of Pennsylvania, Philadelphia, PA 19104-6395 USA; e-mail: ted@math.upenn.edu

Commutative algebra, Irena Peeva, Department of Mathematics, Cornell University, Ithaca, NY 14853 USA; e-mail: irena@math.cornell.edu

Number theory, Henri Darmon

3. GEOMETRIC ANALYSIS \& PDE

Coordinating Editor: Tatiana Toro, Department of Mathematics, University of Washington, Box 354350, Seattle, WA 98195-4350 USA; e-mail: toro@uw.edu

Geometric analysis, Tatiana Toro

Harmonic analysis and partial differential equations, Monica Visan, Department of Mathematics, University of California Los Angeles, 520 Portola Plaza, Los Angeles, CA 90095 USA; e-mail: visan@math.ucla.edu

Partial differential equations and functional analysis, Alexander A. Kiselev, Department of Mathematics, MS-136, Rice University, 6100 Main Street, Houston, TX 77005 USA; e-mail: kiselev@ rice.edu

Real analysis and partial differential equations, Wilhelm Schlag, Department of Mathematics, The University of Chicago, 5734 South University Avenue, Chicago, IL 60637 USA; e-mail: schlag@ math.uchicago.edu

4. ERGODIC THEORY, DYNAMICAL SYSTEMS \& COMBINATORICS

Coordinating Editor: Vitaly Bergelson, Department of Mathematics, Ohio State University, 231 W. 18th Avenue, Columbus, OH 43210 USA; e-mail: vitaly@math.ohio-state.edu

Algebraic and enumerative combinatorics, Jim Haglund, Department of Mathematics, University of Pennsylvania, Philadelphia, PA 19104 USA; e-mail: jhaglund@math.upenn.edu

Probability theory, Robin Pemantle, Department of Mathematics, University of Pennsylvania, 209 S. 33rd Street, Philadelphia, PA 19104 USA; e-mail: pemantle@math.upenn.edu

Dynamical systems and ergodic theory, Ian Melbourne, Mathematics Institute, University of Warwick, Coventry CV4 7AL, United Kingdom; e-mail: I.Melbourne@warwick.ac.uk

Ergodic theory and combinatorics, Vitaly Bergelson

5. ANALYSIS, LIE THEORY \& PROBABILITY

Coordinating Editor: Stefaan Vaes, Department of Mathematics, Katholieke Universiteit Leuven, Celestijnenlaan 200B, B-3001 Leuven, Belgium; e-mail: stefaan.vaes@wis.kuleuven.be

Functional analysis and operator algebras, Stefaan Vaes

Harmonic analysis and complex analysis, Malabika Pramanik, Department of Mathematics, University of British Columbia, 1984 Mathematics Road, Vancouver, British Columbia V6T 1Z2, Canada; e-mail: malabika@math.ubc.ca

Langlands conjectures, Marie-France Vigneras, 8 Rue des Ecoles, 75005 Paris, France; e-mail: marie-france.vigneras@imj-prg.fr

Probability and statistics, Patrick J. Fitzsimmons, Department of Mathematics, University of California, San Diego, 9500 Gilman Drive, La Jolla, CA 92093-0112 USA; e-mail: pfitzsim@ucsd.edu

All other communications to the editors, should be addressed to the Managing Editor, ALEJANDRO ADEM, Department of Mathematics, The University of British Columbia, Room 121,1984 Mathematics Road, Vancouver, B.C., Canada V6T 1Z2; e-mail: adem@math.ubc.ca 
1212 Roelof Bruggeman, Youngju Choie, and Nikolaos Diamantis, Holomorphic Automorphic Forms and Cohomology, 2018

1211 Shouhei Honda, Elliptic PDEs on Compact Ricci Limit Spaces and Applications, 2018

1210 Zhou Gang, Dan Knopf, and Israel Michael Sigal, Neckpinch Dynamics for Asymmetric Surfaces Evolving by Mean Curvature Flow, 2018

1209 Cristian Anghel, Iustin Coandă, and Nicolae Manolache, Globally Generated Vector Bundles with Small $c_{1}$ on Projective Spaces, 2018

1208 Matthew Harrison-Trainor, Degree Spectra of Relations on a Cone, 2018

1207 Alastair J. Litterick, On Non-Generic Finite Subgroups of Exceptional Algebraic Groups, 2018

1206 Anne-Laure Dalibard and Laure Saint-Raymond, Mathematical Study of Degenerate Boundary Layers: A Large Scale Ocean Circulation Problem, 2018

1205 Charles Collot, Type II Blow Up Manifolds for the Energy Supercritical Semilinear Wave Equation, 2018

1204 Rui Palma, Crossed Products by Hecke Pairs, 2018

1203 Xiao Xiong, Quanhua Xu, and Zhi Yin, Sobolev, Besov and Triebel-Lizorkin Spaces on Quantum Tori, 2018

1202 Naiara V. de Paulo and Pedro A. S. Salomão, Systems of Transversal Sections Near Critical Energy Levels of Hamiltonian Systems in $\mathbb{R}^{4}, 2018$

1201 Bernhelm Booß-Bavnbek and Chaofeng Zhu, The Maslov Index in Symplectic Banach Spaces, 2018

1200 Francis Nier, Boundary Conditions and Subelliptic Estimates for Geometric Kramers-Fokker-Planck Operators on Manifolds with Boundaries, 2018

1199 Ramon Antoine, Francesc Perera, and Hannes Thiel, Tensor Products and Regularity Properties of Cuntz Semigroups, 2018

1198 Colette Moglin and J.-L. Waldspurger, La Formule des Traces Locale Tordue, 2018

1197 Stefano Bianchini and Sara Daneri, On Sudakov's Type Decomposition of Transference Plans with Norm Costs, 2018

1196 Nicola Gigli, Nonsmooth Differential Geometry-An Approach Tailored for Spaces with Ricci Curvature Bounded from Below, 2018

1195 Pablo Shmerkin and Ville Suomala, Spatially Independent Martingales, Intersections, and Applications, 2018

1194 A. M. Mason and N. C. Snaith, Orthogonal and Symplectic $n$-level Densities, 2018

1193 James Damon and Ellen Gasparovic, Medial/Skeletal Linking Structures for Multi-Region Configurations, 2017

1192 R. Lawther, Maximal Abelian Sets of Roots, 2017

1191 Ben Webster, Knot Invariants and Higher Representation Theory, 2017

1190 Agelos Georgakopoulos, The Planar Cubic Cayley Graphs, 2017

1189 John McCuan, The Stability of Cylindrical Pendant Drops, 2017

1188 Aaron Hoffman, Hermen Hupkes, and E. S. Van Vleck, Entire Solutions for Bistable Lattice Differential Equations with Obstacles, 2017

1187 Denis R. Hirschfeldt, Karen Lange, and Richard A. Shore, Induction, Bounding, Weak Combinatorial Principles, and the Homogeneous Model Theorem, 2017

1186 Mikhail Ershov, Andrei Jaikin-Zapirain, and Martin Kassabov, Property $(T)$ for Groups Graded by Root Systems, 2017

1185 Jörg-Uwe Löbus, Absolute Continuity Under Time Shift of Trajectories and Related Stochastic Calculus, 2017

For a complete list of titles in this series, visit the AMS Bookstore at www.ams.org/bookstore/memoseries/. 
I SBN 978-1-4704-4102-9

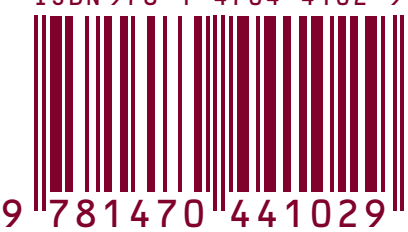

MEMO/255/1218

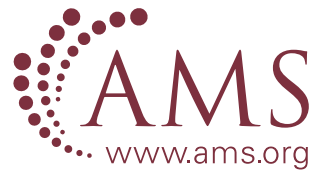

